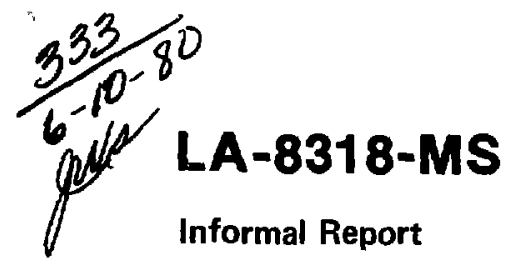

Informal Report

$$
\text { PR. } 1294
$$

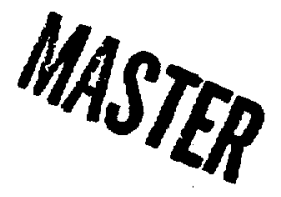

Publications of LASL Research 1978 


\title{
Publications of LASL Research 1978
}

\author{
Compiled by \\ $\because$ J. K. Willis \\ Conchita A. Salazar
}
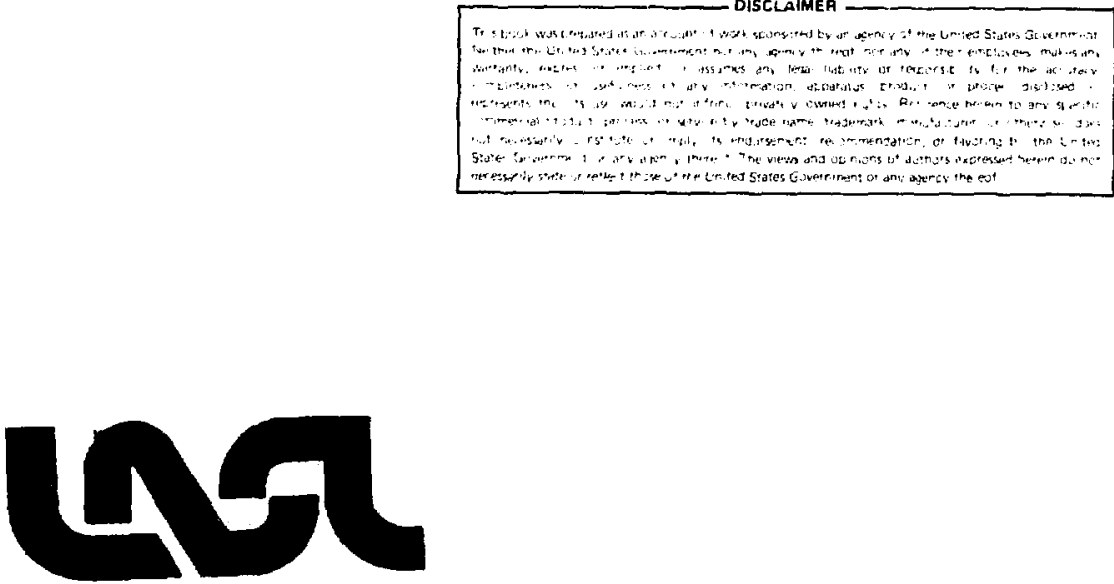
INTRODUCTION. ............................ viii

PREFACE......................... ix

1. Apolications of Explosions.................. I

2. Biology and Medicine....................?

3. Chemical High Explosives................... 10

4. Chemical Separations Processes for Plutonium and Uranium................................ 11

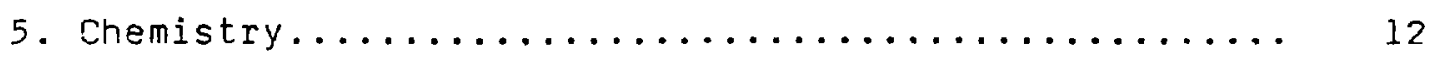

6. Coal Conversion and Utilization - Coal Gasification.. 16

7. Coal Conversion and Utilization--Coal Science

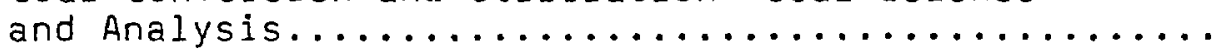

8. Coal Conversion and Utilization--Environmental Protection, Waste Utilization, and Safety...........

9. Criticality Studies...................... 20

10. Electric Energy Systems--Systems

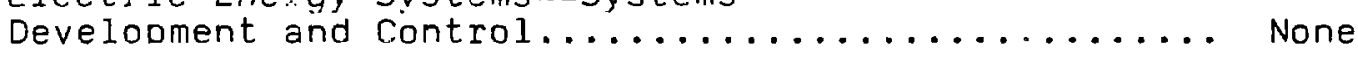

11. Energy Conservation--Electric Power

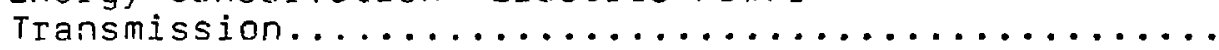

12. Energy Conservation--Systems Modeling

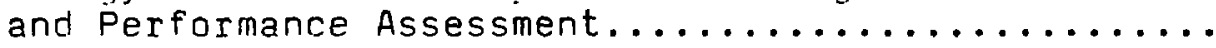

13. Energy Conservation--Waste Systems and

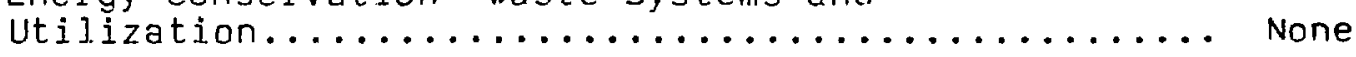

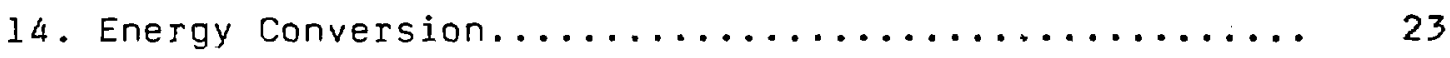

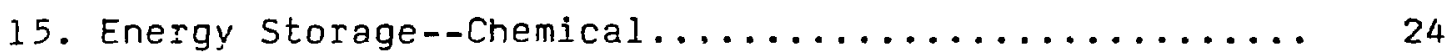

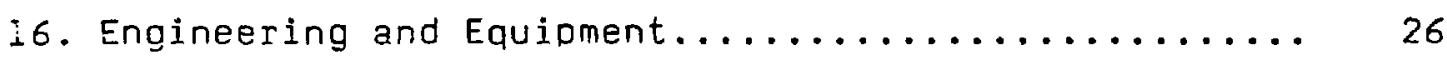

17. Environmental and Safety Analyses.............. 29 
18. Environment Control Technology and Earth

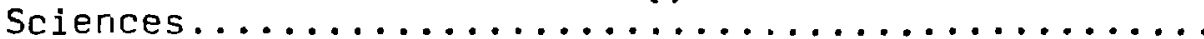

19. Experimental Plasma Physics................. 36

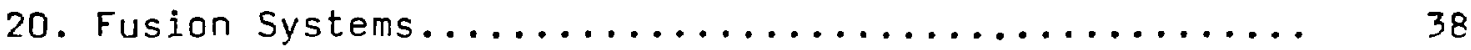

21. Geothermal Eneray--Drilling Technology........... 4].

22. Geothermal Energy--Environmental

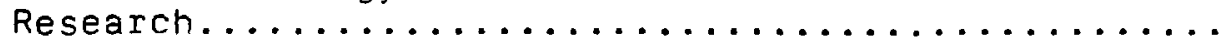

23. Geothermal Energy--Exploration

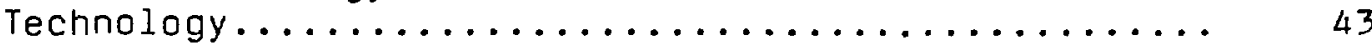

24. Geothermal Energy--Resource Development........... 44

25. Gas Cooled Reactor Technology................ 46

26. General Reactor Technnlogy............... 47

27. General Miscellaneous, and Progress

Reports (Nonnuclear)..................... 40

28 General Miscellaneous, and Progress

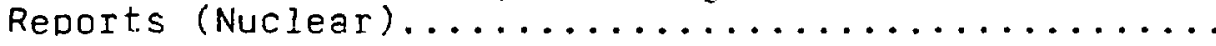

29. Geothermal Energy..................... 52

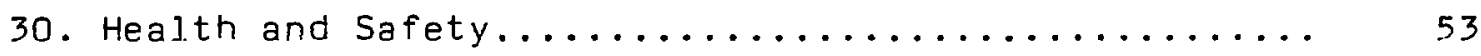

31. Heating and Cooling--Residential

and Commerical Apolications................. $5 \mathrm{f}$

32. Inertial Confinement Fusion............... 58

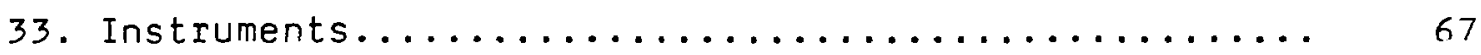

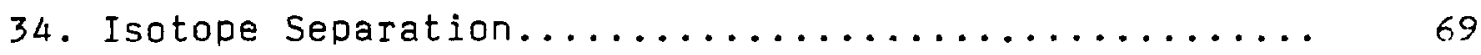

35. Light Water Reactor Technology............... 71

36. Liquid Metal Fast Breeder Reactors:

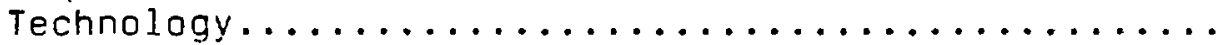

37. LMFBR--Fuels and Materials

Engineering and Development: Base Technology........ 


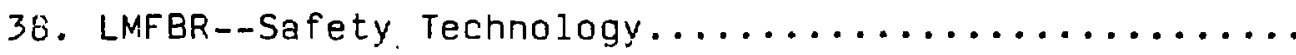

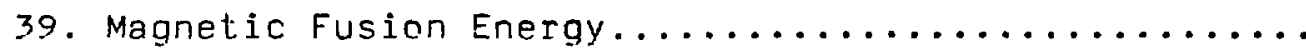

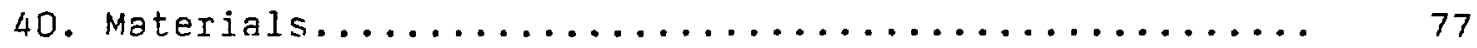

41. Mathematics and Computers................. 81

42. Nuclear Power Sources for Biomedical

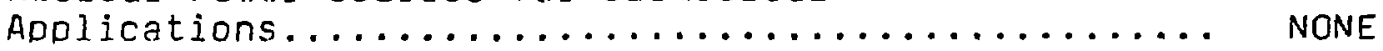

43. Nuclear Propulsion Systems and Aerospace

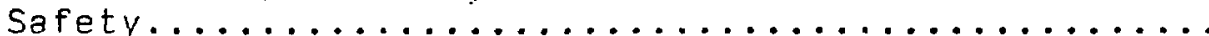

44. Nuclear Raw Materials................... 92

45. Nuclear Waste Management................. 93

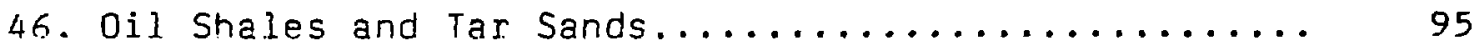

47. Particle Accelerators and High-Voltage

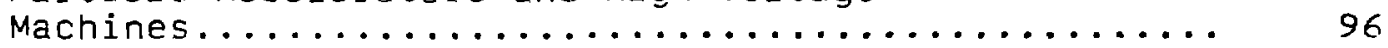

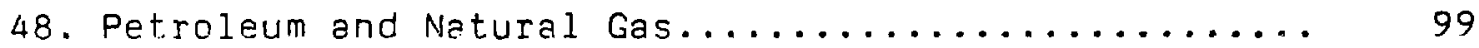

49. Petroleum and Natural Gas Production............ NONE

50. Physjcs--Atomic and Molecular................ 100

51. Physics--Cosmic and Terrestrial.............. 110

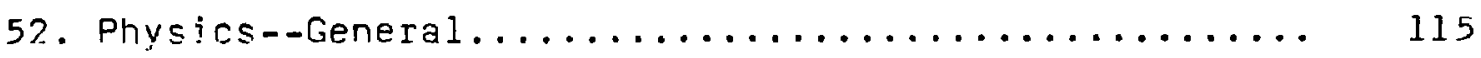

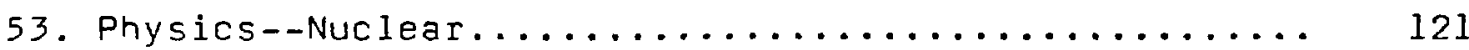

54. Physics--Particles and Fields ............... 131

55. Radioisotope and Radiation Applications........... 133

56. Safeguards--Nuclear Materials Security............ 134

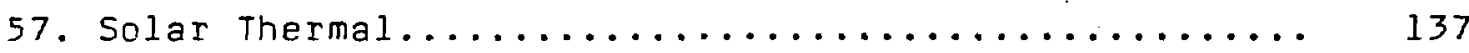

58. Theoretical Plasma Physics.................. 138 
59. Transportation of Property and Nuclear Materials....

Author Index................................ 140

Numerical Index.......................... 203

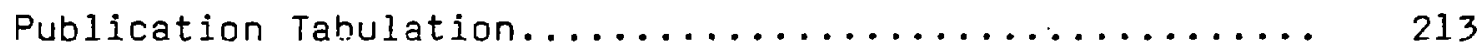

Keyword-in-Context (KWIC) Index......Microfiche in Pocket at the End of the volume. 


\section{INTRODUCTION}

The Los Alamos Scientific Laboratory (LASL), which is operated for the U.S. Department of Energy by the University of California, was founded in 1943 at the site of the Los Alamos Ranch School, a boys' preparatory school in the mountains of northern New Mexico, as part of the wartime Manhatten Project. It had a single assignment--to create the world's first nuclear fission bombs. Success in that task, one of the greatest scientific and technological achievements of all time, was followed by equal success in developing the first thermonuclear weapons. Since 1952, the Laboratory has diversified its programs, and, while still serving as one of the nation's two development centers for nuclear weadons, LASL now devotes about one half of its total effort to unclassjfjed research exploring several peaceful applications of nuclear, conventional and alternative forms of energy. LASL research covers a broad spectrum, ranging from medium and low-energy nuclear physics research to programs involving medical and biological. effects of radiation and basic work in molecular and cellular biology.

Major nonweapons research activities at Los Alamos involve energy research in fields such as superconducting electrical energy transmission and storage, solar and geathermal energy devejooment, laser fusion research and laser isotope separation, and controlled thermonuclear research using magnetic confinement.
Facilities used in such research at the Laboratory include specialized laboratories: a nuclear reactor designed for a variety of experiments: particle accelerators such as the 24-MeV, three-stage Van de Graaff particle accelerator and the 27-MeV electron accelerator desjgned to Droduce a very intense, very short burst of $x$ rays for highspeed flash radiography. At LAMPF, where physics and biomedical experiments are carried out, and where production of a variety of radioisotopes for medical use is under way, an 800 - MeV, I-mA-beam linear accelerator is used to produce heams of protons, mesons, and other nuclear particles with intensities up to 10,000 times greater than those available elsewhere. Another of the Laboratory's facilities, Scyllac, is a toroidal thetapinch device for controljed thermonuclear research involving magnetic confinement of hot plasmas for fusion research. A central Computing Facility with two Cray-1s, four CDC 7600s, two CDC $6600 \mathrm{~s}$, and two CYBER $70 \mathrm{~s}$ assists in the Laboratory's research.

LASL, as of 1978, employed about 6,300 Dersons, about onethird of whom are scientists and engineers. The total operating costs--excluding construction--of the laboratory are about $\$ 275$ million per year. The value of the DOE-owned buildings and equioment at Los Alamos is about $\$ 650$ million. 
The LASL Libraries' continuing Drogram of locating, recording, and announcing publications of LASL-sponsored research has resulted in the previous publications: "Selected Bibliography of Publications of LASL Research, 1957-1962," (LAMS-2895 and supDlement), "Publications of LASL Research," for each of the years 1963 through 1966, "Publications of LASL Research, 1967- -1971," (LA-5400-MS), "Publications of LASL Research, 1972-1976," (LA-6755-MS, Vol. I and Vol. 2) and "Publications of LASL Research, 1977" (LA-7248-MS).

This bibliography is a compilation of unclassified publications of work done at the Los Alamos Scjentific Laboratory for 1978. Papers published in 1978 are included regardless of when they were actually written. Publications received too late for inclusion in earlier compilations have also been listed. Declassification of previnusly classified reports is considered to constitute publication. All classified issuances are omitted--aven those papers, themselves unclassified, which were published only as part of a classified document. If a Daper was published more than once, all places of publication are included.

The bibliography includes Los Alamos Scientific Laboratory reDorts, papers released as nonLASL reports, journal articles, books, chapters of books, conference papers whether published separately or as part of conference proceedings issued as books or reports, papers published in congressional hearings, theses, and U.S. Datents. Publications by LASL authors that are not records of Laboratory-sponsored work are included when the Library becomes aware of them.

The arrangement in each bibliographical citation is title first, place(s) of Dublication indented, then authors, further indented. The CHEMICA: ABSTRACTS abbreviation is used for journal cjtations exced for the capitalization. The group designations given after the authors' names indicate the groups with which the authors were associated at the time the papers were witten. Parenthetical information includes report and reprint numbers, book call numbers, and patent numbers. The parentheses indicate the information by which Los Alamos borrowers may obtain the material from the LASL Libraries.

The entries are arranged in sections by broad subiect categories. Within each section thev are alphabetical by title. All types of publications are interfjled. Some previously used categories have no entries in this compilation. These categories are noted in the Tahle of Contents by the word NONE in olace of a page number.

The author and numerical indexes refer to the item numbers, which consist of a number designating the section, followed by a dash and a number for the alphabetic arrangement of the item within the section. In the numerical index, the $L A$ and LA-x $\times x \times$-MS numbers of formal reDorts are interfiled. 
There is a Keyword-in-Context index in microfiche format in a pocket at the end of the volume.

Most of the reports listed in this publication are available for sale from

National Technical

Information Service

U.S. Department of Commerce

Springfield, VA 22151

or on request by gavernment agencies and their contractors from

Technical Information Center

U.S. Department of Energy

P. D. Box 62

Dak Ridge, TN 37830

Reprints of some journal articles are available as lono as the supoly lasts from the first author listed. Address reauests to the first author at
Los Alamos Scientific

Laboratory

University of California

Post office Box 1663

Los Alamos, NM 87545

This bibliography has been prepared by the LASL Library Services Group, ISD-4, from the records of the classification of fice, from abstracting and indexing journals, and from supdlementary library files. Inquiries regarding any of the materials included should be directed to the Technica]. Processes Section of the LASL Libraries.

The Libraries gratefully acknowledge the assistance of members of LASL's Camputer Science \& Services Division and the Administrative Automatic Data Processing Department for assistance with the computerized system for handing publication information. 
1. APPLICATIONS OF EXPLOSIONS

ROW 1 CHARGE CRATERING CALCULATIONS.

LASL, 23P. (LA-7303-MS)

MALLERA, WALTER $(J-15)$ 
ACCELERATORS FOR PION CLINICAL FACILITIES.

TIC 196 . 16P. MN (LA-UR-76-2083)

ALSO PUBLISHED IN:

INT. J. RADIAT ONCOLOGY BIOL. PHYS.,

V. 3: $\mathrm{P}: 293-7$. ig77: (MP-3)

2T-2 II CELL - CYCLE - SPECIFIC CHANGES IN CHROMATIN ORGANIZATION CORRELATE TEMPORALLY WITH HISTONE H1

PHOSPHORYLATION.

EIOCHIM. BIOPHYS. ACTA,

V. $517, \dot{P}, 486-99: 1978$

HILDEBRAND CARL E: (H-9)

TOBEY, ROBERT A $(\dot{H}-9)$

WALTERS, RONALD A. $(H-9)$

AGE 2 RESPONSE FOR CHO CELLS EXPOSED

TO NEGATIVE PIONS.

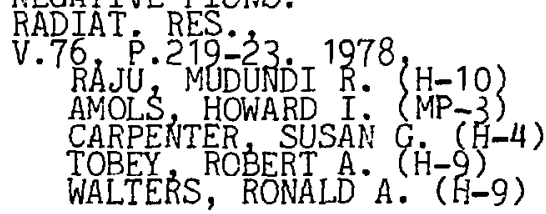

APPL-4CATIONS OF OPTIMAL CONTROL THEORY TO IMMUNOLOGY.

TIC,

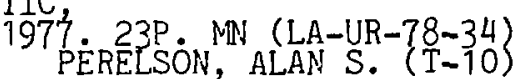

AUTOMATED CANCER - CELL SORTING AND

ANALYSIS, JUNE 1-NOVEMBER 30, 1977.

LASL,

1978: 30P. (LA-7076-PR)

MULLANEY, PAUL F $(\mathrm{H}-10)$

STETNKAMP, JOHN $\dot{A} \cdot(\mathrm{H}-10)$

SALZMAN, GARY C. $(\mathrm{H}-10)$

ZVITRA, ZITA V. $(\mathrm{H}-10)$

CROWELL, JOHN M. $(\mathrm{H}-10)$

MARTIN, JOHN C. $(H-10)$

HANSEN' KAREN $\dot{M}$. ( $(H-10)$

SKOGEN-HAGENSON; MARY JANE $(\mathrm{H}-10)$

HIERERT, RICHARD D. (E-5)

GENTER, $F$. C.
AUTOMATED CANCER - CELL SORTIMG AND ANALYSIS, DECEMBER 1, 1977-MAY 31, 1978.

1978:. 36P. (LA-7412-PR)

MULLANEY PAUL F. $(H-10)$

STEINKAMP, JOHN A. $(\mathrm{H}-10)$

SALZMAN, GARY C. $\left(\begin{array}{l}L S-2 \\ \text { CROWELL, JOHN M. }\end{array}\right.$

SVITRA, ZITA V. $\left(\begin{array}{l}\mathrm{H}-10 \\ \mathrm{LS}-2\end{array}\right)$

$2-7$

AUTORADIOGRAPHIC TECHNIC FOR

PLUTONTUM, POLONIUM AND

PROMETHIUM TISSUE SECTIONS.

J. HISTOTECH.

V. 1 CARPENTER, SUISAN G. $(\mathrm{H}-4)$

2-8

BIOLOGICAL EFFECTS OF NEGATIVE PIONS.

TIC,

1976. 33P MN (LA-UR-76-2529)

ALSO PUBLISHED IN:

INT. J. RADIAT. ONCOLOGY BIOL. PHYS RAJU MUDUNDI R. $(H-10)$

AMOLS, HOWARD I: $(M P-3)$

BAIN, ELVIRA ( $\mathrm{H}-4)$

CARPENTER, SUSAN G. $(\mathrm{H}-4)$

DICELLO, 3OHN $F$. JR. (MP-3)

FRANK, 3. F. ( $\mathrm{H}-10)$

TOBEY, ROBERT A. (H-9)

2-9

BIOMEDICAL AND ENVIRONMENTAL

RESEARCH PROGRAM OF THE LASL HEALTH

DIVISION, JANUARY - DECEMBER 1977.

LASL

1978. 193P. (LA-7254-PR)

PETERSEN, DONALD F. (H-DOT) COMP

SULLIVAN, ELIZABETH M. (H-DO) COA

CALCULATION OF DOSIMETRY PARAMETERS

FOR FAST NEUTRON RADIOTHERAPY.

LASL; 408P. THESIS, TEXAS

AGRTCULTURAL AND MECHANICAL

UNIV., COLLEGE STATION, TX,

1978.' (LA-7288-T)

WELLLS, ALAN HARVEY 
$2-11$

CARBON - 13 AND HYDROGEN -1

NUCLEAR MAGNETIC RESONANCE

STUDIES OF BRADYKININ AND

BIOCHEMISTRY

$$
\begin{aligned}
& \text { V. } 17 \text { P.2 } 270-7.1978 . \\
& \text { LONDON ROBERT E. (CNC-4) } \\
& \text { STEWART, JOHN M. } \\
& \text { CANN JOHN R. } \\
& \text { MATWTYOFF, NICHOLAS A. (CNC-4) }
\end{aligned}
$$

CA $2-12$

CARBON -12 NUCLEAR MAGNETIC

RESONANCE STUDY OF THE CIS-

TRIPEPTIDES

BIOCHEMISTRY,

V. 17 PONDÓN, ROBERT 1978 (CNC-4)

MATWIYOFF NICHOLAS A. (CNC-4)

CANN, JOHN P.

CELL $2-13$ CYCLE - SPECIFIC CHROMOSOME

DAMAGE FOLLOWING TREATMENT OF

CULTURED CHINESE HAMSTER CELLS

WITH $4^{\prime}-((9-A C R I D I N Y L)-$

AMINO METHANESULPHON-M-

ANISIDIDE-HCL.

$$
\begin{aligned}
& \text { J: NAT. CANCER INST. } \\
& \text { DEAVEN LARRY L ( } \\
& \text { OKA MELVIN S. }(\mathrm{H}-10)
\end{aligned}
$$

\section{2-14}

CHARACTERIZATION OF NORMAL AND

NEOPLASTIC MAMMALIAN CELLS BY

MULTIPARAMETER FLOW-SYSTEM

ANALYSIS METHODS.

DETECTION AND PREVENTION OF
CANCER 1976 3RD INT SYMP., NEW

YORK. PROC. PREVENTION AND

DETECTION OF CANCER. H.E. NIEBURGS,

ED. PT $2, V .1, P .1081-9$. DEKKER,

1977. (616.99\% 479P)

ALSO PUBLISHED IN:

TIC

$$
\begin{aligned}
& \text { 1976. } 11 \mathrm{P} \text { MN (LA-UR-76-919) } \\
& \text { STEINKAMP JOHN A }(\mathrm{H}-10) \\
& \text { HANSEN KAREN M. }(\mathrm{H}-10) \\
& \text { CRISSMAN, HARRY A. }(\mathrm{H}-10)
\end{aligned}
$$

COBALT RETENTION PATTERNS IN MICE AS INFLUENCED BY ADMINISTERED DOSE.

\section{8: 4P. (LA-7205-MS)}

THOMAS, ROBERT G. $(\mathrm{H}-4)$

DRAKE, GLESSIE A. $(\mathrm{H}-4)$
2-16

COMBINATION OF SENSITIZERS WITH HIGH

LET RADIATIONS

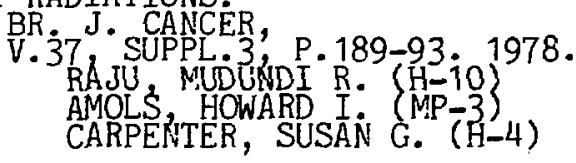

COMPARISON OF SEVERAL COHERENT

OPTICAL TRANSFORM PROCESSING

SYSTEMS FOR CERVICAL CANCER

DETECTION.

LASER ÄND ELECTROOPTICAL SYSTEMS.

1978. OSA/IEEE CONFERENCE,

SAN DIEGC, CA, DIGEST OF

TECHNICAL PAPERS

$$
\begin{aligned}
& \text { P.58. OSA, } 1978 . \\
& \text { (621:36/L34E } 1978) \\
& \text { PEKNICK, B. P. } \\
& \text { WOHLERS, M. R. } \\
& \text { MENDELSOHN, J. } \\
& \text { JOST, S. } \\
& \text { HEROLD, R. } \\
& \text { SALZMAN, GARY C. }(H-10)
\end{aligned}
$$

2-18

CURRENT STATUS OF IMMUNOLOGIC STUDIES IN HUMAN LUNG CANCER.

LASL, 15P. (LA-7371-SR)

GROSS, ROBERT L: (H-4)

DATA ACOUISITION AND DISPLAY

FOR A HIGH - SPEED CELL SORTER.

COMPUT. BIOMED. RES.,

SALZMAN, GARY C. $(\mathrm{H}-10)$

HIEBERT, PICHARD D. $(E-3)$

CFOWELL, JOHN M. $(\dot{H}-10)$

$2-20$

DEGRADATION OF NUCLEIC ACID IN

AQUEOUS SOLUTION BY IONIZING

RADIATIOH. III. THE CORRELATION OF

RADIATION DAMAGE WITH CHANGE IN

MELTING TRANSITION - MODEL

EXPERTMENTS.

BIOPHY S CHEM.

V.8, $\mathrm{P} .123-34$ ' 1978.

HOARD, DÓNALD E. $(\mathrm{H}-9)$

HOLLSTEIN, ULRICH]

GOAD WALTER B. JR. $(\mathrm{T}-10)$

$2-21$

DELAYED RADIATION INJURY OF

GUT - EXPOSED AND GUT - SHIELDED

MICE. II. THE DECREMENT IN

IFE SPAN.

$$
\begin{aligned}
& \text { LASL, } 1978 \text {. } 4 \text { (LA-6717-MS, VOL 2) } \\
& \text { SPALDNG JOHN F. (H-4) } \\
& \text { ARCHULETA, RUBEN F. }(\mathrm{H}-4) \\
& \text { PRINE, JAMES R. (H-4) }
\end{aligned}
$$


2-22

DEPHOSPHORYLATION OF HISTONES H1 AND H3 DURING THE ISOLATION OF METAPHASE CAROMOSOMES.

NUCLEIC ACIDS RES.

V.5 P.3195-208. ¡9ं78
D ANKA, JOSEPH A (H-g)
GURLEY, LAWRENCE R $(\mathrm{H}-9)$
DEAVEN, LARRY L. (H-9)

$2-23$

DETAILED STUDIES ON THE APPLICATION OF THREE FLUORESCENT ANTIBIOTICS FOR DNA STAINING IN FLOW CYTOMETRY.

$197 \%$

$$
\begin{aligned}
& \text { CRISSMAN, HARRY A }(\mathrm{H}-10) \\
& \text { STEVENSON, ANITA }(\mathrm{H}-10) \\
& \text { ORLICKY, DAVID J. }(\mathrm{H}-10) \\
& \text { KISSANE, RICHARD } \mathrm{J}(\mathrm{H}-10)
\end{aligned}
$$

DETECTION OF EARLY CHANGES IN LUNG

CELL CYTOLOGY BY FLOW - SYSTEMS

ANALYSIS TECHNIQUES, JULY 1-

DECEMBER 31, 1977.

$$
\begin{aligned}
& \text { 1978; 7P, (LA-7247-PR) } \\
& \text { STEINKAMP JOHN A }(\mathrm{H}-10) \\
& \text { HANSEN, KAREN M. }\left(\begin{array}{l}
H-10 \\
H-4
\end{array}\right) \\
& \text { SAUNDERS, GEORGE C }(\mathrm{H}-10) \\
& \text { ORLICKY DAVID J. }(\mathrm{H}-10) \\
& \text { CRISSMAN, HARRY } \AA \text {. }(\mathrm{H}-10)
\end{aligned}
$$

DEVELOPMENT OF A CELL - ANALYSIS

AND SORTING SYSTEM APPLICABLE TO

TUMOR CELL CHARACTERIZATION AND

KINETIC STUDIES, OCTOBER 17, 1977APRIL 16, 1978 .

$$
\begin{aligned}
& \text { 1978. 23P. (LA-7300-PR) } \\
& \text { HOFFMAN, ROBERT A, }(\mathrm{H}-10) \\
& \text { SALZMAN, GARY C }(H-10) \\
& \text { BRITT, WILLIAM E. }(E-2) \\
& \text { KISSANE,'RICHARD } \mathrm{J} \text {. ( }(\mathrm{H}-10) \\
& \text { TRAVIS, G. }(\mathrm{H}-10) \\
& \text { JETT, JAMES } \mathrm{H} \text {. }(\mathrm{H}-10) \\
& \text { ORLICKY, DAVID J. }(\mathrm{H}-10) \\
& \text { BUTLER, MILLARD T. }(\mathrm{H}-10) \\
& \text { HORNE, JOHN LOUIS }(H-10) \\
& \text { MULLANEY, PAUL F. }(\mathrm{H}-10)
\end{aligned}
$$

2-26

DEVELOPMENT OF A CELL ANALYSIS AND SORTING SYSTEM APPLICABLE TO TUMOR CELL CHARACTERIZATION AND KINETIC

\section{8}

$$
\begin{aligned}
& \text { 78, 23P, (LA-7583-PR) } \\
& \text { SALZMAN, GARY C (H-10) } \\
& \text { MARTIN, JOHN C. (H-10) } \\
& \text { CRISSMAN, HARRY A. }(\mathrm{H}-10) \\
& \text { KISSANE, RICHARD J }(\mathrm{H}-10) \\
& \text { ORLICKY, DAVID J }(\mathrm{H}-10) \\
& \text { HORNE, JOHN LOUIS }(\mathrm{H}-10) \\
& \text { JOHNSON, TOD S. (H-10) }
\end{aligned}
$$

2-27

DEVELOPMENT OF A PROTON RADIOGRAPHIC SYSTEM FOR DIAGNOSIS AND LOCALIZATION OF SOFT - TISSUE ABNORMALITIES, FINAL REPORT.

LASL, 7P. (LA-7107-MS) HANSON, KENNETH M. (MP-3)

$2-28$

DOMINANT LETHAL ASSAY FOR BIS-DINITR PROPYL-FORMAL, BIS-DINITRO-PROPYLACETAL AND KERIMIDE 601 RESIN.

$$
\begin{aligned}
& \text { LASL, } \\
& \text { 1978. 5P. (LA-7208-MS) } \\
& \text { WILSON, JULIE S. (H-4) } \\
& \text { SMITH, DAVID M. (H-4) }
\end{aligned}
$$

$2-29$

DOSE OUTSIDE THE TREATMENT

VOLUME FOR IRRADIATION WITH

NEGATIVE PIONS.

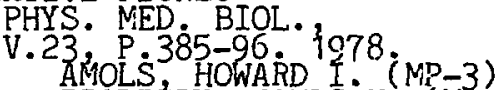

$$
\begin{aligned}
& \text { BRADBURY, JAMES N. (MP-3) } \\
& \text { DICELLO, JOHN F. JR. (MP-3) } \\
& \text { HELLAND, JEROME A. (MP-3) } \\
& \text { KLIGERMAN, MORTON M. (DIR/ADRT) } \\
& \text { LANE THOMAS } F \text { (WX-5) } \\
& \text { PACIOTTI, MICHAEL A (MP-3) } \\
& \begin{array}{l}
\text { POEDER, DENNIS L. (MP-13) } \\
\text { SCHILLACI, MARIO E. (MP-3) }
\end{array}
\end{aligned}
$$

EFFECT

OF NEGATIVE PIONS ON CELLS PL

$$
\begin{aligned}
& \text { RADIATION RES. } \\
& \text { V.75 P. } 339-42,1978, \\
& \text { RAJU, MUDUNDI R. }\{\text { H-10) } \\
& \text { AMOI HOWARD I. HP-3 } \\
& \text { ROBERTSON, JAMES B. (H-10) }
\end{aligned}
$$

$2-31$

EFFECTIVE GAMMA OF TURBULENCE AND GROWTH RATE OF TURBULENCE ENERGY DENSITY RELATIVE TO PRESSURE.

$$
\begin{aligned}
& \text { LASL, } 5 \text { P. (LA-7594-MS) } \\
& \text { BINSTOCCK, JUDITH (TD-2) }
\end{aligned}
$$

ELECTROTHERMAL TECHNIQUE FOR THE ALTERATION OF CORNEAL CURVATURE.

$$
\begin{aligned}
& \text { LASL, 7P. (LA-7155-MS) } \\
& \text { DOSS, JAMES D (MP-3) } \\
& \text { HUTSON RICHARD L. (MP-3) } \\
& \text { ALBILLAR, JOSE I. (MP-3) }
\end{aligned}
$$

ELEV̈ß33 CD CELL - SURFACE HYALURONATE IN SUBSTRATE - ATTACHED CELLS.

EXP 114 CELL RES. $153-1978$ KRAEMER PAÜL M. (H-9)

BARNHART, BENJAMIN J. (H-9) 
$2-34$

EVOLUTIONARY DISTANCE AND SECONDARY STRUCTURE FOR BIOMOLECULES.

1978:

(LA-7153-MS)

WATERMAN MICHAEL S. $(\mathrm{Q}-12)$

\section{2-35}

FLOW - SYSTEMS ANALYSIS AND

CHARACTERIZATION OF PROTEIN

CONTEITS AND PFOLIFERATING KINETICS

IN ASCITES AND SOLID TUMORS.

DETECTION AND PREVENTION OF
CANCER 1976 . 3RD INT. SYMP., NEW

CANCER 1976 . 3RD INT SYMP.
YORK. PROC. PREVENTION AND

DETECTTON OF CANCER. H.E. NIEBURGS

ED ${ }^{\mathrm{PT}}{ }^{1}, \mathrm{~V} \cdot 1, \mathrm{P} .79-90$. DEKKER, 1977. CRISSNAN, HARRY A. $(H-10)$

KISSANE RICHARD J- $(5-10)$

OKA MELVIN S. ( $\mathrm{H}-10)$

STEINKAMP, JOHN A. (H-10)

$2-36$

FLOW ANALYSIS AND SEPARATION

OF EXFOLIATED RESPIRATORY

TRACT CELLS.

TIC, 27P. MN (LA-UR-77-659)

ALSO PUBLISHED IN:

PULSE CYTOPHOTOMETRY

1978. 3RD INTERNAT IONAL SYMPOSIUM,

VIENIIA. PROCEEDINCS, P. 175-85.

EUROPEAN PRESS, 1978

STEINKAMP JOHN A (H-10)

HANSEN, KAREN M. $(\mathrm{H}-10)$

SALZMAN, GARY C. (H-10)

FLOW $2-37$ CYTOMETRY IN BIOLOGY AND

MEDICINE.

LASL

MINI-REVIEW. 1978. 4P. (LASL-78-22)

STEVENSON, ANITA $(\dot{H}-10)$

FLOW MICROFLUOROMETRIC AND

SPECTROPHOTOFLUOROMETRIC

ANALYSIS OF DNA STAINING IN

MAMNALIAN CELLS.

TIC,

CITSPA MN (LA-UR-77-677)

STEVENSON, ANITA $(\mathrm{H}-10)$

KISSANE, RICHARD J. $(\mathrm{H}-10)$
$2-40$

HEAVY PARTICLE COMPARATIVE STUDY.

PART I. DEPTH DOSE DISTRIBUTIONS: V.51 J.P. RADTOL 1033.1978. RAJU, MUDUNDI R. (H-10) AMOLS, HOWARD $I$. $(M P-3)$ DICELLO, JOHIV F. , JR. (MP-3) HOWARD, J. LYMAN J. T. GRAVES R. R. J. B.

HEAVY $2-41$ PARTICLE COMPARATIVE STUDY

PART II. CELL SURVIVAL VERSUS DEPTH.

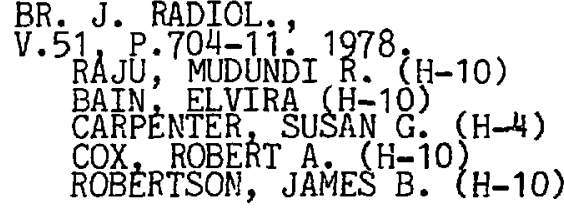

HEA ${ }^{2}-42$ PARTICLE COMPARATIVE STUDY. PART III. OER AND RBE.

BR. J1.P. RADIOL.' 1978. RAJU' MUDUNDI R. $\left(\begin{array}{l}\mathrm{H}-10 \\ \text { AMOLS, HOWARD I: }\end{array}\right.$ AMOLS, HOWARD I:-1 MP CARPENTER, SUSAN G. $(\mathrm{H}-4)$ COX ROBER'T A. $(\mathrm{H}-\mathrm{iO})$ ROBERTSON, JAMES B. ( $\mathrm{H}-10)$

HEAVY PARTICLE COMPARATIVE STUDY. PART IV. ACUTE AND LATE REACTIONS. V.51 J.P. RADIOL.' 1978. CARPENTER, SUSAN G. (H-4)

HISTAMINE. RELEASE DUE TO BIVALENT PENICILLOYL HAPTENS. CONTROL BY THE NUMBER OF CROSSLINKED IGE ANTIBODIES ON THE BASOPHIL PLASMA MEMBRANE.

$$
\begin{aligned}
& \text { J. IMMUNOL. } \\
& \text { V. 121,P.35-8. } 1978 . \\
& \text { DEMBO MICAH (T-10) } \\
& \text { GOLDSTEIN BYRON (T-10) } \\
& \text { SOBOTKA ANNE K } \\
& \text { LICHTENSTEIN, LAWRENCE M. }
\end{aligned}
$$

$2-39$

GENERATION OF AEROSOLS OF

CARCINOGENIC AROMATIC AMINES.

AM. IND. HYG ASSOC. J. ,

RAPPAPORT, STEPHEN $M \cdot(H-5)$

GETTEMY, DONALD J. $(\dot{H}-5)$ 
$2-45$

HISTONE PHOSPHORYLATION AND

CHROMATIN STRUCTURE IN SYNCHRONIZED

MAMMALIAN CELLS.

$$
\begin{aligned}
& \text { CELL CYCLE RÉGULATION. } \\
& \text { J. R. JETER ED. P.37-60. } \\
& \text { ACADEMIC } 1978 \text {. } \\
& \text { GURLEY, LAWRENCE R. (H-9) } \\
& \text { TOBEY ROBERT A. (H-Q) } \\
& \text { WALTERS RONALD A }{ }^{\prime}(\mathrm{H}-9) \\
& \text { HOHMANN, PHILIP G. (H-9) } \\
& \text { D'ANNA, JOSEPH A. }\left\{\begin{array}{l}
H-9 \\
H-9
\end{array}\right. \\
& \begin{array}{l}
\text { BARHAM, STEVEN S. }(\mathrm{H}-9) \\
\text { DEAVEN, LARRY L. }(\mathrm{H}-9)
\end{array}
\end{aligned}
$$

$2-46$

HISTONE PHOSPHORYLATION AND

CHROMATIN STRUCTURE DURING

MITOSIS IN CHINESE HAMSTER

CELLS.

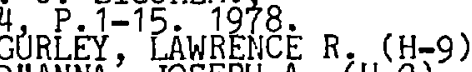

$$
\begin{aligned}
& \text { D"ANNA, JOSEPH A. }\left\{\begin{array}{l}
H-9 \\
H-9
\end{array}\right\} \\
& \text { BARHAM, STEVEN S. (H-9 } \\
& \text { TOBEY, ROBERT A. (H-9) }
\end{aligned}
$$

$2-47$

HISTORICAL INTRODUCTION

TO THEORETICAL IMMUNOLOGY.

THEORET ICAL IMMUNOLOGY

GEORGE I. BEIL, ED. CHAPT . 1, P. $3-41$ MARCEL'DEKKER,

$$
\begin{aligned}
& 1978 \text {. (615.97/B433T) } \\
& \text { BELL, GEORGE T }(T-10) \\
& \text { PERELSON, ALAN S. (T-10) }
\end{aligned}
$$

\section{$2-48$}

IN VITRO MUTAGENICITY TESTING.

1. KERIMIDE 601 RES IN SYLGA RD 184

184 CURING AGENT.

$$
\begin{aligned}
& \text { LASL, } 4 P \text { (LA-7437-MS) } \\
& 1978 \text { WANG, SUSIEY Y }(H-4) \\
& \text { SMITH, DAVID M. }(H-4)
\end{aligned}
$$

INVESTIGATION OF THE MICRODOSIMETRIC CHA RACTERISTICS OF BROAD,

THERAPEUTIC BEAMS OF NEGATIVE

PIONS AT LAMPF.

TIC 197 . 13P. MN (LA-UR-78-1205) DICELLO, JOHN $F_{0}, J R .(M P-3)$
2-50

KINETIC RESPONSE OF CULTURED

CHINESE HAMSTER CELIS TO TREATMENT

WITH $4^{1}-((9-A C R I D I N I L)-A M C N O)$

METHANESULPHON-M-ANISIDIDE-HCL.

J. NAT CANCER INST

V. $60 \mathrm{P} .1147-53.1978$

DEBEY ROBERT A. $\left\{\begin{array}{l}H \\ H\end{array}-9\right\}$

OKA, MELVIN S. (H-10)

$2-51$

LATE BIOLOGICAL EFFECTS OF IONIZING RADIATION AS INFLUENCED BY DOSE, DOSE RATE, AGE AT EXPOSURE, AND GENETIC SÉNSITIVITY TO NEOPLASTIC TRANSFORMATION.

$$
\begin{aligned}
& \text { TIC, } 11 P . \text { MN (LA-UR-78-681) } \\
& 1978 \text { SPALDING JOHN F }(H-4) \\
& \text { PRINE, JAMES R. }(\mathrm{H}-4) \\
& \text { TIETJEN, GARY L. }(\mathrm{Q}-12)
\end{aligned}
$$

LIGHT ${ }^{2}$ T2 SCATTER ANALYSIS OF

MICROALGAE. CORRELATION OF SCATTER

PATTERNS FROM PURE AND MIXED ASYNCHRONOUS CULTURES.

$$
\begin{aligned}
& \text { BIOPHYS. J } \\
& \begin{array}{l}
\text { KOLLMAN, VICTOR H, }\left(\mathrm{H}^{-1} 1\right) \\
\text { SALZMAN, GARY C. }(\mathrm{H}-10)
\end{array}
\end{aligned}
$$

LIMITATIONS OF THE JERNE HEMOLYTIC PLAOUE TECHNIOUE

THEORETICAL IMMUNOLOGY

GEORGE I. BELL ED. CHAPT 44. (6) $16.97 \%$ B $433 \mathrm{~T})$ GOLDSTEIN, BYRON $(T-10)$

2-54

LOS ALAMOS SCIENTIFIC

LABORATORY ION LINEAR

ACCELERATOR PROGRAM.

TIC,

$1976.9 P . M N$ (LA-UR-76-2082)

AISO PUBLISHED IN:

INT. J. RADIAT ONCOLOGY BIOL. PH KNAPP EDWARD A: (MP-3) SWENSON, DONALD A. (MP-14)

2-55

LYMPHOCYTE TRAFEIC PATTERNS AND CELL - CELL INTERACTIONS

THEORETICAL IMMUNOLOGY

GEORGE I. BEL ED. CHAPT 12 P. 341-75: MARCELL DEKKER, 1978. (¿ 
$2-56$

MODELS OF THE EVENTS RESPONSIBLE FOR ANTIBODY PRODUCTION BY

B LYMPHOCYTES. TMMUNOCY THEORET ICAL IMMUNOLOGY

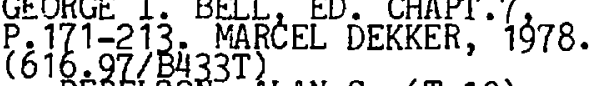
PERELSON, ALAN S. (T-10)

MULT'T2 2 PARAMETER ANALYSIS AND SORTING OF MAMMALIAN CELLS.

YEAR BOOK OF CANCER 1975.

ALSO PUBLISHED Î:

EXP. CELL RES.' 1974.

STEINKAMP JOHN A. $(\mathrm{H}-10)$

ROMERO, ANGELA $(\mathrm{H}-10)$

HORAN, PAUL $\mathrm{K}$. ( $\mathrm{H}-10)$

CRISSMAN, HARRY A. $(\mathrm{H}-10)$

\section{$2-58$}

NEW METHODOLOGY FOR MEASURING

DAMAGE TO RESPIRATORY TRACT

CELLS USING FLOW - SYSTEM

SENSING OF ENVIRONMENTAL

POLLUTANTS 1978 . JOINT CONF

4TH, NEW ORLEANS. PROC. ' P.52

STEINKAMP JOHN A (H-TO)

HANSEN, KAREN M. $(H-10)$

WILSON, JULIE S. (H-4)

SALZMAN, GARY C: (H-10)

2-59

OPTIMAL STRATEGIES IN

IMMUNOLOGY . II. B MEMORY

CELL PRODUCTION

J. MATH. BIOL.' 1978.

PERELSON ALART S (T-10)

OSTER, GEORGE F.

\section{2-60}

FICOSECOND AND STEADY STATE,

VARIABLE INTENSITY AND VARTẢBLE TEMPERATURE EMISSION SPECTROSCOPY

OF BACTERTORHODOPSIN.

B.23 P. 383-93. 1978.

SAAPIRO, STANLEY L. (AP-4)

LEWIS, A:

PERREAULT, G.

SLAYTON, R'.
$2-61$

PICOSECOND PULSE TRAIN STUDY OF EXCITON

DYNAMICS IN PHOTOSYNTHETIC MEMERANES.

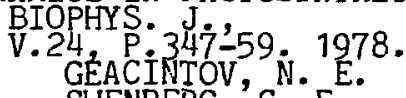

SWENBEPG, C. E.

CAMPILLO; ANTHONY J- $(A P-4)$

SHAPIRO, STANLEY L. (AP-4)

WINN, KENNETH

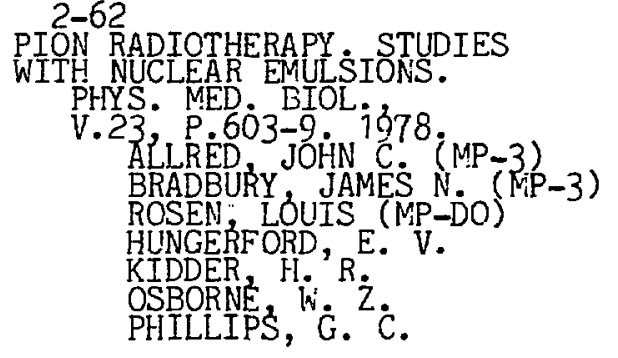

PRELIPIINARY TOXICOLOGICAL STUDY OF BIS-DINITRO-PROPYL-FORMAL.

BIS-DINITRO-PROPYL-ACETAL.

LASI

1978' 5P. (LA-7206-MS)

SMITH, DAVID M. (H-4)

DRAKF, GLESSIE A. $(\mathrm{H}-4)$

LONDUN, JERRY E. ( $\mathrm{H}-4)$

WILSCN, JULIE S. $(\mathrm{H}-4)$

HOLLAND, LAURENCE M. (H-4)

THONAS, ROBERT G. (H-4)

2-64

PRELIMINARY TOXICOLOGICAL STUDY OF KERIMIDE 601 RESIN.

LASL,

(LA-7207-MS)

SMITH, DAVID M. (H-.4)

DRAKE; GLESSIE A. $(\mathrm{H}-4)$

LONDON, JERRY E. (H-4)

WILSON, JULIE S: $\{\mathrm{H}-4\}$

HOLLAND, LAURENCE M. $(\mathrm{H}-4)$

THOMIAS, ROBERT G. ( $\dot{H}-4)$

2-65

PRELIMINARY TOXICOLOGICAL STUDY OF

SILASTIC 386 FOAM ELASTOMER.

LASL

1978: 3P. (LA-7365-MS)

SMITH, DAVID M. $(H-4)$

LONDON, JERRY E. ( $\mathrm{H}-4)$

DRAKE, GLESSIE A. $\left\{\begin{array}{c}H-4 \\ \mathrm{H}-4\end{array}\right\}$ 


\section{2-66}

PRELIMINARY TOXICOLOGICAL STUDY OF SILASTIC 386 FOAM ELASTOMER -

CATALYST.

1978

SMITH; DAVID M. (H-4)

DRAKE; GLESSIE A. $(\mathrm{H}-4)$

LONDONA, JERRY E. $(\mathrm{H}-4)$

2.-67

PRELIMINARY TOXICOLOGICAL STUDY OF SILASTIC 386 CATALYST.

$$
\begin{aligned}
& \text { LASL, } \\
& \text { 1978. 5P. (LA-7367-MS) } \\
& \text { SMITH, DAVID M. (H-4) } \\
& \text { DRAKE' GLESSIE A. (H-4) } \\
& \text { HOLLAND, LAURENCE M. (H-4) } \\
& \text { JACKSON, DOUGLAS E }(\mathrm{H}-10) \\
& \text { LONDON, JERRY E. (H-4) } \\
& \text { PRINE, JANES R. (H-4) } \\
& \text { THOMAS, ROBERT G. }(\mathrm{H}-4)
\end{aligned}
$$

\section{2-68}

PRELIMINARY TOXICOLOGICAL STUDY OF SYLGARD 184 CURING AGENT.

LASL

197

SMITH, DAVID M. $(H-4)$
LONDON, JERRY E. $(H-4)$
DRAKE, GLESSIE A. $\left(\begin{array}{l}H-4 \\ H-4\end{array}\right.$ THOMAS, ROBERT G.

2-69 SYLGARD 184 ENCAPSULATING RESIN.

LASL

$$
\begin{aligned}
& \text { 1978: 3P. (LA-7369-MS) } \\
& \text { SMITH, DAVIDM. (H-4) } \\
& \text { DRAKE, GLESSIE A. }(\mathrm{H}-4) \\
& \text { LONDON, JERRY E. }(\mathrm{H}-4) \\
& \text { THOMAS, ROBERT G. }(\mathrm{H}-4)
\end{aligned}
$$

\section{2-70}

PRELIMINARY TOXICOLOGICAL STUDY OF SYLGARD 184 ENCAPSULATING RESIN CURING AGENT.

LASL

$$
\begin{aligned}
& \text { 1978, 3P. (LA-7370-MS) } \\
& \text { SMITH, DAVID M. (H-4) } \\
& \text { DRAKE; GLESSIE A. (H-4) } \\
& \text { LONDON, JERRY E. }(H-4) \\
& \text { THOMAS, ROBERT G. }(H-4)
\end{aligned}
$$

PRODUCTION AND PROPERTIES OF RADIOACTIVE MICROSPHERES FOR LOCALIZED IRRADIATION OF

LUNG TISSUE

$$
\begin{aligned}
& \text { HEALTH PHYS. } \\
& \text { V. } 34 \text { P. } 25-36.1978 . \dot{C} \text {. } 1 \text { H-DO }) \\
& \text { AIIDERSON, ERNEST C. }(\mathrm{H}-10) \\
& \text { PERRINGS, JAMES D. }
\end{aligned}
$$

$2-72$

PRODUCTION, RECOVERY, AND

PURIFICATION OF HAF NIUM - 172 FOR UTILIZATTON IN NUCLEAR MEDTCINE

AS THE GENERATOR OF LUTETIUM -172.

INT. J. NUCL. MED. BIOL.,

V. 5, P. 11-7. 1978.

DANIELS, REBECCA J. (CNC-11)

GRANT ${ }^{\text {"BRIEN }}$, HAROLD A., JR. (CNC

PROTON COMPIJED TOMOGRAFHY.

BULL. AM P PHYS SOC.,

HANSON, KENNETH M. (MP-3)

PULSED SYSTEM FOR OBTAININC

MICRODOSIMETRIC DATA WITH HIGH

INTENSITY BEAMS

IEEE TRANS. NUCL. SCI.,

V. NS-25, P. 202-4. 1978.

ALSO PUELLISHED IN:

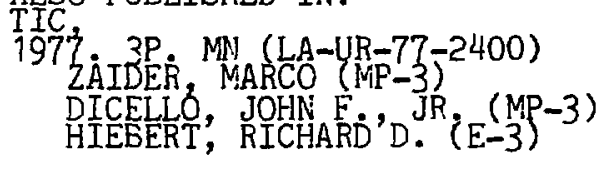

2-75 75 TATION OF CELL FUSION BY TWENTY ONE STRAINS OF NEWCASTLE DISEASE VIRUS USING FLOW

MICROFLUOROMETRY.

$$
\begin{aligned}
& \text { J. GEN. VIROL: } \\
& \text { V. } 41 \text { CRAM } 27-36: 1978 . \\
& \text { CRAMSUND SCOT' }(\dot{H}-10) \\
& \text { JETT, JAMES H. }(\dot{H}-10)
\end{aligned}
$$

$2-76$

RADIATION EXPOSURE TO RODENTS INHABITING A RADIOACTIVE WASTE RECEIVING AREA.

$$
\begin{aligned}
& \text { HEALTH PHYS: } \\
& \text { V. } 34 \text { P } 603-9 \text {. } 1978 . \\
& \text { MIERA FELIX R. JR. }(\mathrm{H}-8) \\
& \text { HAKONSON, THOMÁS E. }(\mathrm{H}-8)
\end{aligned}
$$

RAPID RADIOIODINATION OF

ROSE BENGAL AT ROOM TEMPERATURE.

$$
\begin{aligned}
& \text { J. NUCL. MED. } \\
& \text { V. } 19 \text {, 'P }: 525-\dot{9}: 1978 \\
& \text { HúPF, } \mathrm{H}_{\text {. E }} \text { (CNC-11) } \\
& \text { O"BRIEN, HAROLD A. JR. (CNC-1 } \\
& \text { HOLLAND, LAURENCE } 3 \text {. }
\end{aligned}
$$


2-78

SEQUENTIAL BIOCHEMICAL EVENTS IN THE CELL CYCLE

CELL CULTURE CONGRESS. 1975 INTERNATIONAL, BIRMINIGHAM, AL. PROC. CELL CULTURE AND ITS

APPLICATION. P.5-22. R.T. ACTON, ED.

ACADEMIC, 1977 . (574.8/C $393 \mathrm{C})$

TOBEY, ROEERT A. (H-9)

WALTERS, RONALD A. $\left\{\begin{array}{l}\mathrm{H}-9 \\ \mathrm{H}-9\end{array}\right)$

GILDEBRAND, CARL E. $\left(\begin{array}{l}H-9 \\ H-9\end{array}\right)$

SEROLGGIC TEST SYSTEMS DEVELOPMENT, JULY 1, 1976-SEPTEMBER 30, 1977.

L.AST

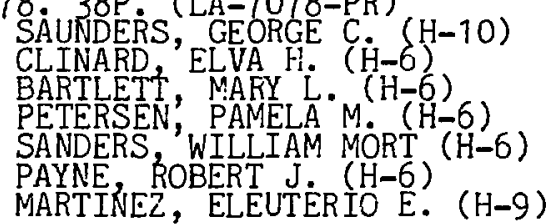

$2-80$

SIMGULAR PEFTURBATION APPROACH

TO DIFFUSION REACTION EQUATIONS

CONTAINING A POINT SOURCE, WITH

APPLICATION TO THE JERNE HEMOLYTIC

PLAQUE ASSAY.

$$
\begin{aligned}
& \text { J. MATH. BTOL. } \\
& \text { V. } 9 \text { P. } 75-85.978 . \\
& \text { PERELSON, ALAH S. (T-10) } \\
& \text { SEGEL, LEE A. }
\end{aligned}
$$

2-81

SPATIAL DISTRIBUTION! OF SUREACE IMMUNNOGLOBUL IN ON B LYMPHOCYTES. LOCAL ORDERING.

$$
\begin{aligned}
& \text { EXP CELL RES } \\
& \text { V. } 12 \text { P } 309-211978 \\
& \text { PERELSON, ALAN } 3 .(T-10)
\end{aligned}
$$

SYNOPSIS OF RESEARCH ON RNA

SECONDARY STRUCTURE.

$$
\begin{aligned}
& \text { LASL, } 5 \text { P. (LA-753S-MS) } \\
& \text { WATERMAN, MICHAEL S. (S-1) }
\end{aligned}
$$

\footnotetext{
2-83 SYMMETRIC BIVALENT HAPTENS TO CELL SURFACE ANTIBODY - APPLICATION TO HISTAMINE RELEASE FROM BASOPHILS.

J. I I 1 MMUNOL P. $345-53.1978$.

DEMEO MTCAH $(T-10)$

GOLDSTEIN, BYRON $(T-10)$
}

2-84

THEORY OF EQUILIBRIUM BINDING OF ASYMMETRIC BIVALENT HAPTENS TO CELL SURFACE ANTIBODY APPLICATION TO J. IMMUNOL.

V. 121 P. $5 \dot{3} 3-601.1978$. WOESY CARLA GOLDSTEIN, BYRON (T-10) DEMEO, MICAH (T-10)

2-85

THERMAL TREATMENT TECHNIQUE FOR CANCER EYE.

1978. 5P. MN (LA-UR-78-636) DOSS, JAMES D. (MP-3)

THREE DOSE COMPONENTS OF A NEGATIVE PION BEAM AND THEIR ROLE IN PION RADIOTHERAPHY RADIOLOGY,

V. 128 P $P 757-66.1978$. RODRIGUEZ JOE F $(\mathrm{E}-1)$ MALCON, JACK E. (E-1) STEIN, MYRON L. ( $\mathrm{C}-3)$

2-87

TRATAMIENTO ELECTROTERMICO DEL CANCER DEL OJO.

LASL

MINI-REVIEW, 1978. 4P.

(LASL-77-14) DOSS, JAMES D. (MP-3)

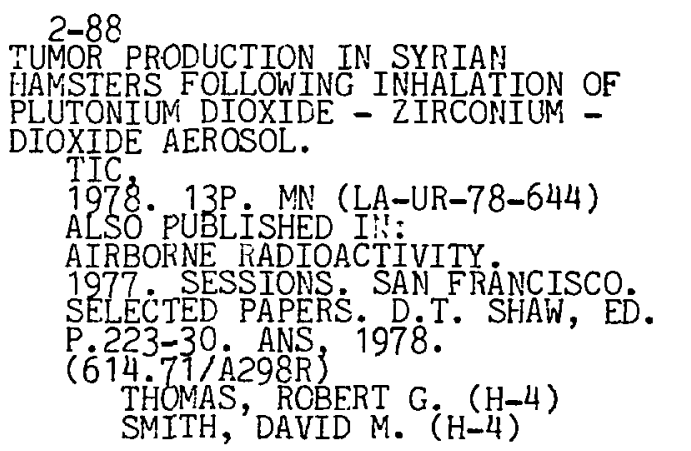

X-IPRADIATION-INDUCED NUCLEAR LESIONS IN CULTURED MAMMALIAN CELLS - AN ULTRASTRUCTURAL ANALYSIS.

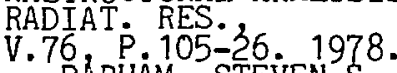
BARHAM, STEVEN S. $(\mathrm{H}-9)$ 
3. CHEMICAL HIGH EXPLOSIVES

BURNING AND DETONATION. 1978. 41P. (LA-7245) FOREST; CHARLES A. (T-14)

CHX 3 RACTERIZATION OF TWO COMMERCIAL EXPLCSIVES.

\section{LASL, 19P. (LA-7140)}

CRAIG BOEBY GLENN $(E-5)$
JOHNON JAMES N. $(T-14)$
MADER, CHARLES L. (T-144)
LEDERMAN, CORDON F., JR. (M-3)

3-3

PENATION SENSITIVITY TESTS.

LASL, 41P. (LA-7193-MS)

URIZAR MANUEL J. (WX-2)

PETERSON, SUZANNE $W \cdot(W X-2)$

SMITH, LOUIS C. (WX-2)

DETONATION VELOCITY OF PETN IN SMALL CONFINED CYLINDRICAL CHARGES.

PROPELLANTS EXPLOS.,

V. ${ }^{\text {DINEGAR, ROBERT }}$ H. (WX-7)

EXPLO - EXPLOSIVES THERMAL ANALYSIS COMPUTER CODE.

1978. 4OP. AND APPEND.

(LA-6949-MS) JAEGER, DWIGHT L. (WX-3)

FIBER - REINFORCED PLASTIC - BONDED EXPLOSIVES.

LASL, 38P

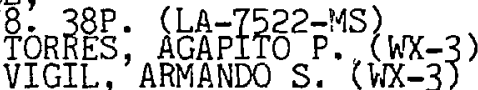

HI ${ }^{3-7}{ }^{7}$ - TEMPERATURE SHOCK INITIATION

OF EXPLOSIVES.

LASL, 22P. (LA-7158)

RAMSAY, JOHN B. (M-3)

CRAIG, BOBBY GLENN (M-DO)

DICK, JERRY JOEL $(M-3)$
$3-8$

IGNITION AND DEFLAGRATION OF

POTASSIUM PICRATE (KP) AND KP EXPLOSIVES MIXTURES. LOW VOLTAGE, NONPRIMARY DETONATORS.

TIC, 17P. MN (LA-UR-78-1298) DINEGAR, ROBERT H. $(\overline{W X}-7)$

IGNITION AND INITIATION OF POTASSIUM PICRATE AND POTASSIUM PICRATE/EXPLOSIVES MIXTURES NONPRIMARY, HOT - WIRE DETONATORS. LASL, 1978: 10P. (LA-7358-MS) DINEGAR, ROBERT H. (WX-7)

$3-10$

MODEL FOR THE PRECIPITATION OF PENTAERYTHRITOL

TETRANITRATE (PETN)

I\&EC PRCCESS DESIGN \& DEV.,

V. 17 P P $182-8.1978 . \dot{T}$ THOMAS RANDOLPH, ALAN D.

$3-11$

NUMERICAL MODELING OF THE DEFLAGRATION-TO-DETONATION TRANSITION.

TIC
$1978.21 P . M N(L A-U R-78-913)$
MADER, CHARLES L. (T-14)

PREDICTION OF LOW CYCLE FATIGUE FAILURE IN A 20,000 PSI PRESSURE TASEL

CUBE SYMPOSIUM. 1978 . LOS ALAMOS,

NM. ABST. J.J RUMINER, COMP RUMINER, JOHN J: (WX-3) NEUDECKER, JOSEPH W. (WX-8)

SAFE ${ }^{3-13}-$ STATIONARY DETONATION TRAIN F ARMY ORDNANCE.

LASL

1978. 5P. (LA-7123-MS)

GOFORTH, JAMES H. (WX-7)

$3-14$

TECHNIQUES FOR MEASUREMENT OF

DENSITIES CF PBX SAMPLES AT OTHER THAN ROOM TEMPERATURE.

1978: 4P. (LA-7450-MS) MARTINEZ, CARLOS $\stackrel{\text { A. }}{\text { M }}(\mathrm{WX}-3)$ 
4. CHEMICAL SEPARATIONS PROCESSES FOR PLUTONIUM AND URANIUM

\section{4-1}

METHDD FOR PHOTOCHEMICAL REDUCTION

OF URANYL NITRATE BY TRI-N-BUTYL

PHOSPHATE AND APPLICATION OF THIS

METHOD TO NUCLEAR FUEL REPROCESSING.

PATENTED

MAR. 21, 1978, FILED 1976.

(U.S, PATENT-4,08O, 2 Z 3 ) (AP-3)

REFER-DE POORTER, CHERYL K. (AP-3) 


\section{$5-12$ \\ DECOMPOSITION OF HYDROGEN BROMIDE \\ USING IRON BROMIDE AND MAGNETITE. \\ TIC \\ MASON, CAROLINE F. V. (CMB-3)}

\section{DETERMINATION OF ARSENIC AND}

\section{GALLIUM IN STANDARD MATERIALS BY} ACTIVATION ANALYSIS.

ANAL. CHIM. ACTA

V. 96 P. $271-7$. 1978.
WANGEN, LAWRNCE $(\mathrm{H}-8)$
GLADNEY, ERNEST S. $(\mathrm{H}-8)$

$5-14$

DETERMINATION OF ARSENIC, ANTIMONY, MOLYBDENUM, THORIUM, AND' TUNGSTEN IN SIIIICATES BY THERMAL NEUTRON ACTIVATION AND INORGANIC ION EXCHANGE.

ANAL. LETT.
V. 11 P $42 \dot{9}-35$ 1978.
GLADNEY, ERNEST S.

$(H-8)$

$5-15$

DETERMINATION OF URANIUM AND

NEPTUNIUM IN PLUTONIUM BY X-RAY

FLUORESCENCE.

$$
\begin{aligned}
& \text { LASL, 5P. (LA-7373) } \\
& 1978 \text { MARTELL, CAL (CMB-1) } \\
& \text { HANSEL, JMES M. (CMB-1) }
\end{aligned}
$$

\section{5-16}

EFFECTS OF SAMPLE SURFACE ON ION MICROPROBE ANALYSIS OF PLATINUM RHENIUM - TUNGSTEN ALLOYS.

MICROBEAM ANALYSIS SOCIETY.

ANNUAL CONF 1975. 10TH, LAS

VEGAS NV PROC 2 P 73 AA-73D. THE
SOCIETY 1975. (545\%26A 1975$)$.
HAKKILA, E. ARNOLD (CMB-1)

HANSEL, JAMES M. (CMB-1)

HUTCHINSON, WILLIAM B. (CMB-1)

\section{5-17}

EVOLUTION OF HYDROGEN FROM

FERROUS BROMIDE SOLUTION AND

MAGNESIUM OXIDE.

J. INORG NUCL. CHEM.

MASON CAROLINE F. V. (CMB-3)

BOWMAN, MELVIN G. (CMB-DO)

DAVID, MARY ANN (CMB-3)

\section{$5-18$}

FUNDING OF RESEARCH IN

THERMODYNAMICS

$$
\begin{aligned}
& \text { J. CHEM. ENG DATA, } \\
& \text { V. } 23 \text { HOLLEY, CHARLES E., JR. (CNC-2) } \\
& \text { HOLLE }
\end{aligned}
$$

5-19

CAS PHASE CARBON - 13 CHEMICAL SHIFTS ANI COUPLING CONSTANTS

IN THE DEUTEROMETHANES.

J. CHEM. PHYS., 1978

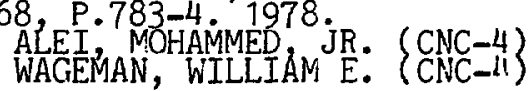

5-20

HIGHLY SELECTIVE COULOMETRIC METHOD AND EQUIPNENT FOR THE AUTOMATED DETERMINATION OF PLUTONIUM.

ANALYTICAL CHEMISTRY IN ENERGY TECHMOLOGY - 1977 21 ST CONF., GATLINBURG; TN. PAPERS ANALYTICAL CHEMISTRY IN NUCLEAR FUEL REPROCESSING. P. 151-8 W. S. LYON, ED SCIENCE PRESS, $1978.0626 / C$ C $48 A P$ 21ST) JACKSON, DARRYL D. (CMB-1) HOLLEN, ROBERT M. ( CMB-1) RCENSCH, F. R. (CME-1)
REIN, JAMES E. (CME-1)

5-21

HYD ROOGEN EVOLVING REACTIONS - THE THERMAL DECOMPOSITIONS OF CEROUS

$$
\begin{aligned}
& \text { J. INORG } \text {. NUCL. CHEM. } \\
& \text { PETERSON, EUGENE J. ( }(\mathrm{CMB}-8) \\
& \text { JOHNSON, N. R. (CME-8) } \\
& \text { BCWNAAN, MELVIN G. (CME-DO) }
\end{aligned}
$$

$5-22$

IRREVERSIEILITY ANALYSIS CF HYDROGEN SEPARATION SCHEMES IN THERMOCHEMICAL CYCLES.

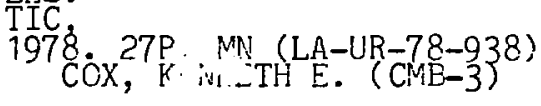

$5-23$

LABILITY AND REACTIVITY OF SULFUR DIOXIDE TRANSITION METAL COMPLEXES. CRYSTAL AND MOLECULAR STRUCTURE OF THE BIS(CHLOROFORM) SOLVATE OF CHLOROHYDRIDO ( SULFUR DIOXIDE)

CARRONYL
PHOSPHINE) BIS(TRICYCL DIUM(II).

$$
\begin{aligned}
& \text { INORG. CHEM. } 1978 \\
& \text { RYAN, ROBERT R. ( } \mathrm{CNC}-4) \\
& \text { KUBAS, GREGORY J. (CNC-4) }
\end{aligned}
$$


$5-24$

MEASUREMENT OF SPECIAL NUCLEAR MATERIAL CONCENTRATIONS IN SOLUTION BY

ABSORPTION EDGE DENSITOMETRY.

T977. 10P MN (LA-UR-77-2235)

ALSO PUBLISHED IN:

ANALYTICAL CHEMISTRY IN ENERCY

TECHNOLOGY. 1977 21ST CONE.,

GATLINBLRG, TN PAPERS.

WUEL REPROCESSING. P $313-21$.

1978. (626/C748AP 21ST)

TAPE, JAMES W. $\quad Q-1\}$

LANGNER, $D$. G. $(Q-1)$

CANADA; THOMAS F. $(Q-1)$

MODELS FOR THE SPECIFIC ADHESION

OF CELLS TO CELLS.

S. 200, P. 618-27. 1978.

BELL, GEORCE I. (T-DO)

$5 \cdot-26$

NTTROGEN - 15 CHEMICAL SHTFTS FOR

IMIDAZOLE IN AQUEOUS CADMIUM 2+

SOLUTIONS.

INORG. CHEM.

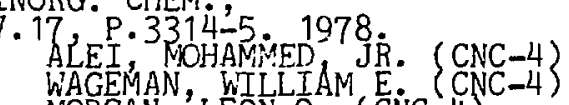

MORGAN, LEON O. (CNC-4)

ON THE INTERPRETATION OF CARBON -13

SPIN LATTICE RELAXATION RESULTING FROM RING PUCKERING IN PROLINE.

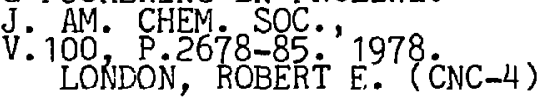

\section{$5-28$}

PHASE RELATIONSHIP, VAPORIZATION,

AND THERMODYNAMIC PROPERTIES OF

THE LANTHANUM - BORON SYSTEM.

$\mathrm{J} \cdot$ PHYS. CHEM.

. 82 P. $51-2.1978$. STORMS, EDMUND K. (CME-3)

$5-29$

PLUTONIUM - OSMIUM, ETA-

PLUTONIUM( 19)OSMIUM.

ACTA CRYSTALLOGR

V. B34, P. $913-5 .{ }^{19} 78$.

$5-30$

PLUTONTUM -239 HALF LIFE

DETERMINED BY ISOTOPE DILUTION

MASS SPECTROMETRIC MEASUREMENT

OF GROWN - IN URANIUM -235 .

INT. J. APPL RADIAT. ISOT., MARSH SINCLAIR F: (CMB-1) ABERNATHEY, ROBERT M. (CMB-1)

BECKMAN, RICHARD ZEIGLER, ROYAL K. $(Q-12)$

PLUTONIUM-PLATINUM PUZPT. ACTA CRYSTALLOGR., V.34B, P. 2608-10:' 1978. CROMER, DON T: (CMB-5)

\section{EPARATION AND CHEMICAL CHARACTERIZATION OF PLUTONIUM - 239 METAL USED FOR HALF - LIFE MEASUREMENT. INT. J. APPL. RADIAT. ISOT., QP. $485-7$. 1978. REIN, JAMES E. (CMB-1) WATERBURY, GLENN R. (CMB-1)}

$5-33$

PREPARATION OF CARBON -

CAREON - 13 DISULFIDE AND CARBON CAREON - 13 OXYSULFIDE.

J. LABELLED COMPD RADIOPHARM. ,

V. 14 P. $793-5$. 1978
KERR, VERNON $\mathrm{H}$. $(\mathrm{H}-11)$
OTT, DONALD G. $(\mathrm{H}-11)$

$5-34$

PREPARATION OF SYNTHETIC STANDARD MINERALS.

$$
\begin{aligned}
& \text { LASL, } \\
& \text { 1978: 50P (LA-7072-MS) } \\
& \begin{array}{l}
\text { HERRICK CLAUDE C ( }(\text { CMB-8) } \\
\text { BUSTAMANTE S. J. ( CMB-DO) } \\
\text { CHARLES, ROBERT W. (CNC-11) }
\end{array} \\
& \text { COWAN, ROBERT E }(E-2) \\
& \text { HULL, DONALD E. (CMB-7) } \\
& \text { OLINGER BARTON W. (M-6) } \\
& \text { ROOF, RAYMOND BRADLEY, JR, (CMB-E } \\
& \begin{array}{l}
\text { SHEINEERG } \\
\text { HERRICK, HASK }
\end{array}
\end{aligned}
$$

5-35

PREPARATION OF WORKING CALIBRATION AND TEST MATERIALS - PLUTONIUM OXIDE

1978

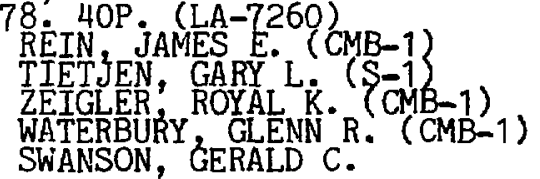


5-36

PREPARATION OF WORKTNG CALIBRATION AND TEST MATERIALS - MIXED OXIDE.

LASL.

1978: 42P , (LA-7322)

REIN JAMES E. (CMB-1)

2EIGLER' ROYAL $\mathrm{K} \cdot(\mathrm{S}-1)$

WATERBURY, GLENN"R. (CMB-1)

PREPĀ 3 TIVE ROUTES TO CEROUS

HYDROXY CARBONATE.

J. INORG NUCL. CHEM. ,

PETERSON, EUGENE $j$. ( CMB-8)

ONSTOTT, EDWARD I. (CMB-8)

$5-38$

PRCCEDURE FOR THE ANALYSIS OF

AMERICIUM IN COMPLEX MATRICES.

LASL,

KNAB, DARYL
KH-8)

5-39

QUIC - A CHEMICAL KINETICS CODE

FOR USE WITH THE CHEMICAL

EQUILIBRIUM CODE QUIL.

LASL

1978 . 49P (LA-NUREG-6998)
LUNSFORD, JOHN L. (CMR-8)

\section{$5-40$}

STRUCTURAL PHASE TRANSFORMATIONS

AND TEMPERATURE DEPENDENT RAMAN

SPECTRA OF CSLLIFE( CN)6.

J. 69 PHEM. $4328-34.1978$

SWANSON, BASSIL I.

LUAAS, ROBERT R. ( CNC-4)

$5-41$

SYNTHESIS OF

4-(2-HYDROXY ETHYLSULFONYL ) - 1-

NAPHTHALENESULFONAMIDE.

J. $O R G$. CHEM.
V. $43.4659-62.1978$.

WHALEY, THOMAS W. $(H-11)$

$5-42$

SYNTHESIS, PROPERTIES, AND STRUCTURE

F IODOSULFINATE SALTS.

INORG. CHEM.-1 1978

ELLER, P. GARY (CNC-4)
KUBAS, GREGORY J. (CNC-4)
$5-43$

THERMOCHEMICAL PRODUCTION OF HYDROGEN FROM W'ATER.

TIC 21P. MN (LA-UR-78-652)
COX KENNETHE (CMB-3)
BOWMAN, MELVIN G. (CME-DO)

$5-44$

THERMOCHEMICAL PRODUCTION OF
HYDROGEN - REALITY, NOT MYTH

$$
\begin{aligned}
& \text { LASL, } \\
& \text { 1978. 6P, (LA-74 15-MS) } \\
& \text { COX, KENNETH E. (CMB-3) }
\end{aligned}
$$

THERMODYNAMIC MODEL OF BINDING OF FLEXIBLE BIVALENT HAPTENS

TO ANTIBODY.

IMMUNOCHEMISTRY,

V. 15 , P. 307-13:' 1978.

DEMBO NICAH $(T-10)$

$5-46$

THERE COORDINATION IN METAL. COMPLEXES

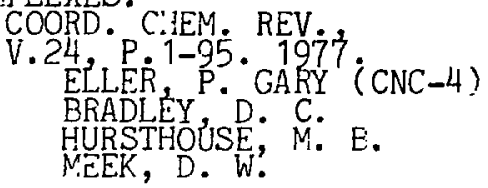


6. COAL CONVERSION AND UTILIZATION-.-COAL GASIFICATION

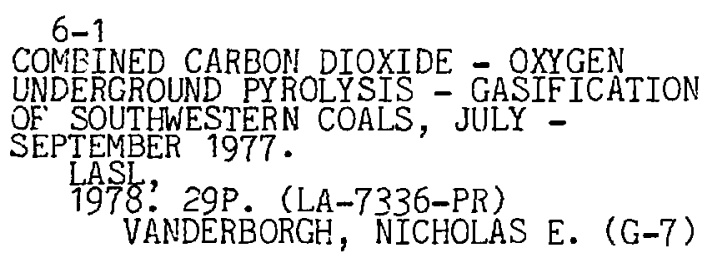

$6-2$

CONCURRENT HEAT AND MASS TRANSFER

DURING DRYING OF BLOCKS OF

SUBBITUMINOUS COALS.

UNDERGROUND COAL CONUERSION

SYMP. 1978 (JULY). 4TH, STEAMBOAT

SPRINGS, CO. FROC: , P.453-60.

SANDIA LABS. , 1978 '.

VANDERBORGH, NICHOLAS E; (G-7)
ELIIOTT, GUY R. E $(G-7)$
CORT, GEORGE E: (WX-4)

$6-3$

LABORA TORY STUDIES OF COAL

DHYING, PYROLYSIS, AND COMBUSTION

FOK UC

SOCIETY OF PETROLEUM ENGTNEERS

OF ATME ANNUAL FALL TECH IICAL CONF.
AND EXHIBITION 1978 (OCT). 53RD,

HOUSTON. PREPRINT SPE 7522:

8P. AIME, 1978

ELLTOOTT GUY R. B. (G-7)
VANDERBORGH, NICHOLAS E.

$(G-7)$

$6-4$

UNDERGROUND THERMAL GENERATION OF

HYDROCARBONS FFOM DRY, SOUTHWESTERN

COALS

EVERGY CONVERSION ENGINEERING

CONFERENCE. 1978. (AUG.) $13 \mathrm{TH}$

INTERSOCIETY, SAIN DIECO, CA. PROC.,

V. 1 P $366-72$ SAE, 1978.

AISSO PUBLISHIED IN:

T978. 8P. MN (LA-UR-78-1438)

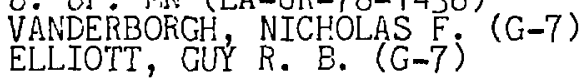


7. COAL CONVERSION AND UTILIZATION--COAL SCIENCE AND ANALYSIS

$\mathrm{COM}^{7}-1$

HEAT AND MASS TRANSFER CODE

CLOSELY MODELS LABORA TORY DATA

OBTA INED FROM DRY ING COAL BLOCKS

AT LASL

UNIVERSITY COAL RESEARCH. 1978

(AUG.) ANNUAL DOE/FOSSIL ENERGY

CONF. 5TH, LEXINGTON. RESEARCH

ACCOMPLISHMENTS. P.67. DOE, 1978 ;
VANDERBORGH, NICHOLAS E. (G-7)

$7-2$

HIGH - TEMPERATURE CARBON DIOXIDE /

OXYGEN (2) - COAL REACTION RATE

EXPERIMENTS.

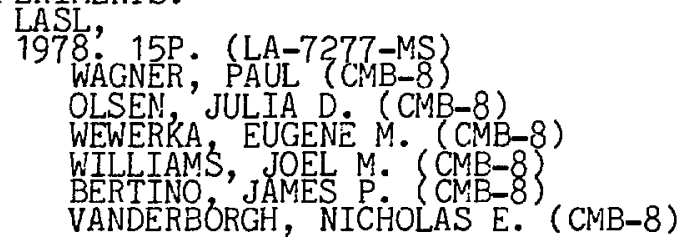

LASL,

WAGNER; PAUL (CMB-8)

OLSEN, JULIA D. (CMB-8)

WEWERKA, EUGENE M. (CMB-8)

WILLIAMS, JOEL M. (CMB-8)

VANDERBORGH, NICHOLAS E. (CMB-8)

\section{$7-3$}

GILLETTE, WYOMING, AND COMMUNITIES

SOUTH AND EAST.

LASL

MORRIS; DONALD W. (S-2)

$7-4$

MASS TRANSFER CHANGES INDUCED IN

COAL BLOCKS DURING THERMAL

PROCESSING.

TIC,

$$
\begin{aligned}
& \text { 13P. MN (LA-UR-78-1183) } \\
& \text { VANDERBORGH NICHOLAS E. } \\
& \text { BERTINO, JAMES P. (CME-8) } \\
& \text { HOPKINS; D. N. }(G-7)
\end{aligned}
$$

7-5

REVIEW OF ORGANIC SULFUR ANALYSIS IN

COAL AND A NEW PROCEDURE.

$$
\begin{aligned}
& \text { TI } \\
& 19 \\
& \text { AL } \\
& \text { SC } \\
& \text { SYM } \\
& \text { V. } \\
& \text { AD } \\
& \text { TE } \\
& \text { AP } \\
& \text { MIC } \\
& \text { PIC }
\end{aligned}
$$$$
\begin{aligned}
& 19778 \text {. } 14 P \text { MN (LA-UR } \\
& \text { ALSO PUBLISHED IN: }
\end{aligned}
$$$$
\text { SCANNING ELECTRON MICROSCOPY/ } 1978 .
$$$$
\text { SYMPOSIUM, LOS ANGELES. PROCEEDINGS, }
$$$$
\text { V. 1. INTERNATIONAL REVIEW OF }
$$$$
\text { ADVANCES IN INSTRUMENTATION }
$$$$
\text { TECHHIQUES THEORY AND PHYSICAL }
$$$$
\text { APPLICATIONS OF THE SCANNING ELECTRON }
$$$$
\text { P.93-108. SEM, } 1978 \text {. }
$$$$
\text { RAYMOND, ROEEET, JR. (G-6) }
$$

GOOLEY, RONALD C. $(\dot{G}-6)$ 
8. COAL CONVERSION AND UTILIZATION--ENVIRONMENTAL PROTECTION WASTE UTILIZATION AND

$8-1$

COAL COMBUSTION. SOURCE OF TOXIC

ELEMENTS IN URBAN AIR?

J.A ENVIRON SCI. HEALTH, GLADNEY, ERNEST S. (H-8)

GORDON, GLEN E.

ZOLLER, WILLIAM $\mathrm{H}$.

$8-2$

CONPUTER SIMULATION OF THE VISUAL

EFFECTS OF SMOKE PLUMF S. THE

ATMOSPHERE. 1978. CONFERENCE,

WASHINGTON, D.C. PROCEEDINGS,

SPIE 1978 .

WILLIAMS, MICHAEL D.

WECKSUNG, MONA J. $\left\{\begin{array}{l}\mathrm{Q}-12 \\ \mathrm{Q}-12\end{array}\right\}$

$8-3$

CONTAMINANTS IN COALS AND CCAL

RESIDUES.
1976. 24P.MN (LA-UR-76-2197)
ALSO PUBLISHED IN:
ENERGY AND THE ENUIRONMENT.
1976 4TH NATIONAL CONFERENCE,
AICHE, 1976 . (614.7/Ë56E 1976$)$
WIILIAMS, JOEL M. (CMB-8)
VANDERBORGH, NICHOLAS E. (CME-8)

$8-4$

ELEMENTAL DEPOSITION DOWNWIND OF A

COAL FIRED POWER PLANT.

WATER, AIR, SOIL POLLU.,

V. $10, P .33-44$. 1978.

WANGEN, LAWRENCE: E. $(\mathrm{H}-8)$

WILLIAMS, MICHAEL D.

8-5

OBSERVATIONS ON BORON RELEASE

FROM COAL FIRED POWER PLANTS.

ENVIRON. SCI. TECHNOL.,

V. $12, P .1084-5.1978$.

GLADNEY, ERNEST S. $(\mathrm{H}-8)$
WANGEN, LAWRENCE E. $(\mathrm{H}-8)$
CURTIS, DAVID B. $(\mathrm{H}-8)$

JURNEY, EDWARD $\dot{T}$. $(\mathrm{P}-\mathrm{DO})$

8-6

PHYSICS RELATED PROBLEMS OF

COAL EIRED POWER PLANT POLLUTION.

PHYS. TEACHER 1978.

DEVANEY, JOSEPH J. (L-5)
8-7

TRACE ELEMENT CHARACTERIZATION AND

REMOVAL / RECOVERY FROM COAL AND

COAL WASTES, JANUARY 1-MARCH 31, 1977 1978: 26P. (LA-7104-PR)

WEWERKA, EUGENE M. (CMB-8)

WILLIAMS, JOEL $M$. (CMB-8)

VANDERBORGH, NICHOLAS E. (G-7)

WAGNER, PAUL (CMB-8)

WANEK, PATSY L. $($ CMB-8 $)$

8-8

TRACE ELEMENT CHARACTERIZATION AND REMOVAL / RECOVERY FROM COAL AND COAL WASTES, APRIL 1-JUNE 30, 1977.

1978: 30P. (LA-7117-PR)

WEWERKA, EUGENE M. (CMB-8)
WILLIAMS, JOEL M. (CMB-8)
VANDERBORGH, NICHOLAS E. (G-7)
HARMON, ANTHONY W. (CMB-1)
WANEK, PATSY L. (CMB-8'
OLSEN, JULIA D. (CMB-8

TRA-9 ELEMENT CHARACTERIZATION OF COAL WASTES - FIRST ANNUAL REPORT, JULY 1, 1975 TO JUNE 30, 1976.

1978. 52P. (LA-06835-PR)

WEWERKA
WILLIAMS, JOEL M. (CMB-8)

8-10

TRACE ELEMENT CHARACTERIZATION OF COAL WASTES - SECOND ANNUAL PROGRESS REPORT - OCTOBER 1, 1976 - SEPTEMBER 1977

LASL, 144P. (LA-7360-PR)

WEWERKA; EUGENE M. (CMB-8)

WILLIAMS JOEL M. (CME-8)

VANDERBOKGH, NICHOLAS E. (G-7)

HARMON, ANTHONY W. (CMB-1)

WAGNER, PAUL (CMB-8)

WANEK, 'PATSY L.: $\begin{aligned} & \text { CMB-8 } \\ & \text { OLSEN, JULIA } \\ & \text { D. }\end{aligned}$ 
8-11

TRACE ELEMENT DISTRIBUTIONS IN

SEVERAL COAL CONVERSION PROCESSES.

AN EXCHANGE PROGRAM BETWEEN THE

LOS ALAMOS SCIENT IFIC LABORATORY

AND THE NATIONAL COAL BOARD OF

ENGLAND.

1978

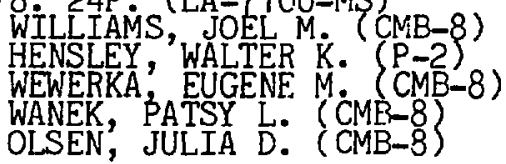


9. CRITICALITY STUDIES

9-?

EXPERIMENTAL CRITICALITY

SPECIFTCATIOONS. AN ANNOTATED

BIBLIOGRAPHY THROUGH 1977.

1978: 32P. (LA-7170-MS)

PAXTON, HUCH C. $(Q-14)$ COMP.

a -2

REACTIVITY COEFFICIENTS OF HEAVY

ISOTOPES IN LASL'S FAST CRITICAL

ASSEMBLIES

TRANS. AM. NUCL. SCC.,

V. 28, PY ${ }^{295-6}{ }^{29} 1978$.

HANSEN, CORDON E- (0-14)

KOELLING JERRY J. ( $(\omega X-8)$

PLASSMANA EUGENE A $(Q-14)$

SMITH, DAVID R. $(Q-i 4)$

THIRTY YEARS AT PAJARITO CANYON SITE.

LASL, 55P. (LA-7121-H)

PAXTON, HUGH C. $(Q-14)$ 
11. ENERGY CONSERVATION--ELECTRIC POWER TRANSMISSION

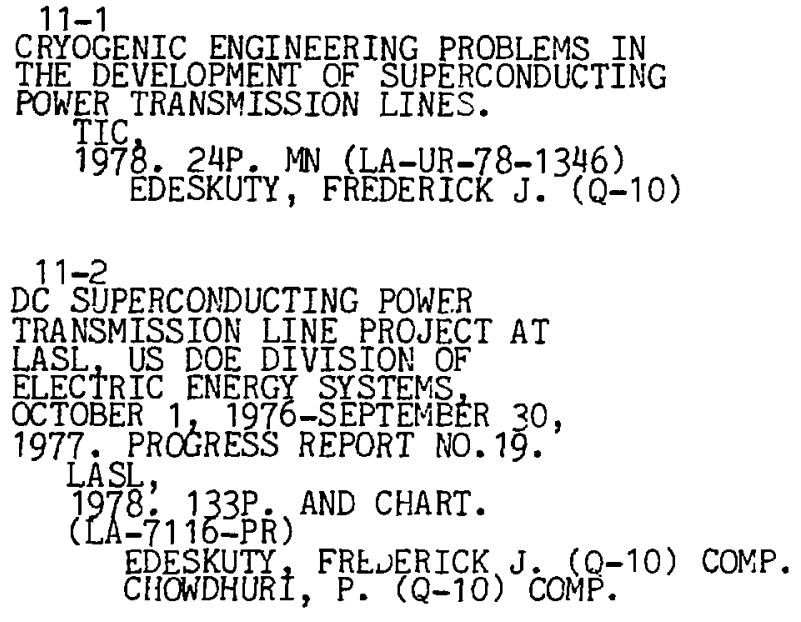

DIRECT CURRENT SUPERCCNDUCTING POWER TRANSMISSION.

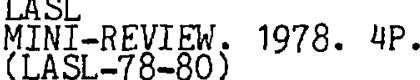

KELLER, WILLIAM E. (Q-10)

\section{$11-4$}

PROPOSED 30-MJ SUPERCONDUCTING

UNIT FOR STABILIZING AN ELECTRIC

TRANSMISSTON SYSTEM.

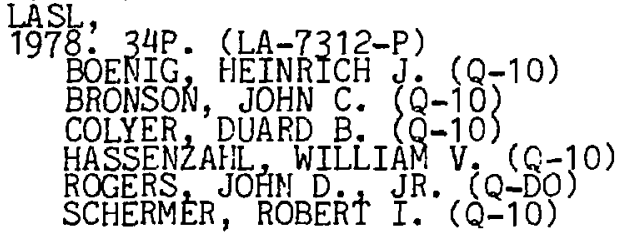

SOME ELECTRICAL CHARACTERISTICS

OF A DC SUPERCONDUCTING CABLE.

TIC,

1977 . 11P. MN (LA-UR-77-199)

ALSO PUBLISHED IN:

IEEE TRANS, POWER APPAR. SYST.,

. PAS-97, $P$. $399-408.1978$

LAQUER, HÉNRY L. $(Q-26)$

$11-6$

SUPERCONDUCTIVITY AND ITS

APPLICATION IN THE ELECTRIC POWER

INDUSTRY.

LASL

MINI-REVIEW. 1978. 4P.

(LASL-78-79) WILLIAM E. (Q-10)
THE-TMOELASTIC ANALYSTS OF A SUPRRCONDUCTING CABLE. LASL,

1978. 2OP. P(LA-7465-MS)

USE. 11 F SUPERCONDUCTIVE TECHNOLOGY

FOR ENERGY STORAGE AND POWER

TRANSMISSION FOR LARGE POWER

SYSTEMS - POWER PARKS.

TIC KELLER, WILLIAM E. $\left(\begin{array}{l}\text { (L-10) } \\ \text { KER }\end{array}\right.$

50-KA FLUX PUMP FOR THE

SUPERCONDUCTING TRANSMISSION LINE TEST BED. LASL

1978.' 11P. (LA-6953-MS) 
12. ENERGY CONSERVATION--SYSTEMS MODELING AND PERFORMANCE ASSESSMENT

$12-1$

SAVING ENERGY - A NEW COMPUTER

LASL

MINII-REVIEW. 1978. 4P. (LASL-78-28)

TUCKER, EVA F. (WX-4) 
14. ENERGY CONVERSION

14-1

CRITICAL REVIEW AND ASSESSMENT OF

PROBLEMS IN HYDROGEN ENERGY DELIVERY

SYSTEMS. INITIAL REPORT NOVEMBER

1 , 1976 -SEPTEMBER 30, 1977.

1978: 69P. (LA-7405-PR)

EDESKUTY, FREDERICK J. (Q-10) COMP. 
15. ENERGY STORAGE--CHEMICAL

15-1
ELECTRICITY GENERATTON AND STORAGE
FOR RESIDENCES USING LITHIUM /L/SUB 2 \% ELECTROCHEMICAL ENGINES TO AUGMENT PIYOTOVOLTA ICS.

TI 977 . OP. MN (LA-UR-77-2330) ELLIOTT, GUY R. B. (CNC-2)

$15-2$

ELECTROCHEMIICAL ENGINES AT UCC SITES FOR ELECTRIC POWER GENERATION AND STORAGE.

UNIVERSITY COAL RESEARCH. 1978.

(AUG.) ANAUAL DOE FOSSIL ENERGY

ACCOMPLISHMENTS. P. 125, DOE, 1978.

15-3

ELECTROCHEMICAL, ENGINES FOR POWER

GENERATION AND LCAD-LEVELING AT

SITES FOR UNDERGROUND COAL

CONVERSION.

ENERGY CONVERSION ENGINEERING

CONFERENCE. 1978. (AUC.) 13T1?

INTERSCCIETY, SA DI DIEGO, CA. PROC.,

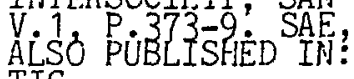

1978

8P. NN (LA-UR-78-1402)

EALIOTT GUY R. B. (G-7)

$15-4$

LIQUID HYDROGEN STORAGE AND

TRANSMISSION.

HYDROGEN - ITS TECHNOLOGY AND

IMPLICATIONS. K.E. COX AND K.D.

WILLIAN SON, EDS. $\dot{V} .2$, TRANSMISSION

AND STORAGE P.51-81. CRC PRESS,

1977 ( $546,1 / C 877 H$ V.2)

EDESKUTY FREDERIC $\dot{C}$ J. (Q-10)

WILLIAMSON, KENNETH D., JR. (CTR-9)

$15-5$

OPTIMIZATION STUDIES FOR THE DESIGN OF SUPERCONDUCTING MAGNET IC ENERGY

STORAGE VACUUM VESSELS.

CUBE SYMPOSIUM. 1978. LOS ALAMOS,

IM ADST J.J. RUMINER COMP

P.98. LA SL, 1978 (LA-7417 BFNDETT, JOEL G. $(Q-13)$

$15-6$

STATUS OF EUROPEAN THERMOCHEMICAL

HYDROGEII PROGRAMS.

TIC 7 . 5P. MN (LA-UR-77-2985)

FDWMAN, MELVIN G. (CME-DO)
$15-7$

STRUCTURAL DESIGN FOR A 10-GWH SMES ACUUM VESSEL

J. PRESSURE VESSEL TECH.,

V. 100 P. 263-70. 1978 .

ALSO PUBLISHED IN:

1978

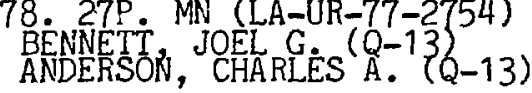

15-8

STRUCTURAL DESIGN FOR A 10-GWH SMES VACUUM VESSEL.

$$
\begin{aligned}
& \text { LASL } \\
& \begin{array}{l}
\text { 78. 35P. (LA-7 110-MS) } \\
\text { BENIETT, JOEL G. (Q-13) } \\
\text { ANDERSON, CHARLES A. (Q-13) }
\end{array}
\end{aligned}
$$

$15-9$

SUPERCONDUCTING MAGNETIC ENERGY STORAGE.

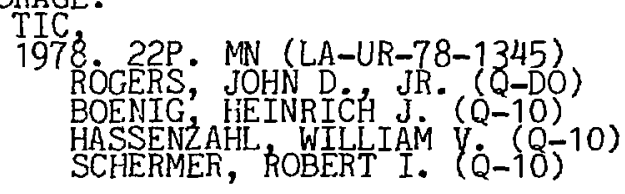

15-10

SUPERCONDUCTING MAGNETIC ENERGY

STORAGE PROJECT AT LASL, JANUARY 1DECEMBER 31, 1976.

LASL,

978: 71P. (LA-7132-PR)

HASSENZAHL WILLIAM V. $(Q-10)$ CO

BOENIG, HEINRICH J. $(\dot{Q}-10)$

BRONSON, JOHN C. $(\dot{Q}-10\}$

COLYER, DUARD B $(\mathrm{Q}-10)$

TURNER, ROBERT D. $(Q-10)$

SCHERMER, ROBERT I. (Q-10)

15-11

SUPERCONDUCTING MAGNETIC ENERGY

STORAGE PROJECT AT LASL JANUARY 1JUNE 30, 1977.

LASI

28P, (LA-7150-PR)

HASSENZAHL WILLIAM V. $(Q-10)$ CO BOENIG, HEINRICH J. (Q-10) BRONSON JOHN C. (Q-10) HASSENZAHL WILLIAM V. $(Q-10)$ SCHERMER
TURNER, ROBERT D. $(Q-10)$ 
15-12

SUPERCONDUCTING MAGNETIC ENERGY

STORAGE (SMES) PROGRAM, JANUARY 1-

DECEMBER 31, 1977.

1978, 41P. (LA-7294-PR)

ROGERS; JOHN D., JR. (Q-DO) COMP.

15-13

SUPERCONDUCTING MAGNETIC ENERCY

STORAGE.

LASL

MINI-REVIEW. 1978. 4P.

(LASL-78-81

KELLER, WILLIAM E. (Q-10)

$15-14$

THERMOCHEMICAL PROCESSES FOR

HYDROGEN PRODUCTION, AUGUST 1, 1977-FEBRUARY 28, 1978.

1978. 12P. (LA-7234-PR)

COX, KENNETH E. (CMR-3) COMP.

BOWMAN, MELVIN G (CMB-DO)

FARR, JOHN D. (CMB-3)

COX KENNETH E (CME-3)

PETERSON, CHARLES L. (CMB-3) 
$16-1$

AUIALOG 4-MHZ FIBER - OPTICS LINK. LASL

1978 NÖ 10P (LA-7377-M)

$16-2$

ANALYSIS CF VENTILATION SYSTEMS

SUBJECTED TO TORNADO CONDITIONS,

MAY 1-SEPTEMEER 30, 1977.

LASL,

1978' 114P. (LA-7550-MS)

ANDRAE, FICHARD W. $(W X-8\}$

DUERRE, KENNETH H. (WX-8)

DOVE, RICHAFD C. $(Q-13)$

SMITH, MARK H.
GREGORY, WILLIAM S. (WX-8)

$16-3$

DESIGN AND TESTS OF A CONTROL SYSTEM FOR THYRISTORIZED PChER SUPPLIES FOR

SUPERCONDUCTING COILS.

ENGINEEFING PROBLEMS OF FUSION

RESEARCH, 1977. SYMP. 7TH,
KNOXVILLE, TH. PROC.; V.1,
P.4Q4-8 M. S LUEELL' ED.

IEEE 1277 . ( 53 TSSSY

RANNEN,, H.

$16-4$

DOE-1 VERIFICATION PROGRAM PLAN.

LASL, 13P. (LA-7552-MS)

DIAMOND STEPHEN C $(W X-4)$

HUNN, ERUCE, D. $(W X-4)$
MC DONALD, THOMAS E. (E-4)

$16-5$

ELECTROCHEMICAL HEAT ENGINE.

PATENTED 1978 , FILED 1977.

(U.S.PATENT-3,994,796)

ELLIOTT, GUY R: E. (G-7)

HOLLEY, CIARLES E. , JR. (CNC-2)

HOUSEMAN, EARTON L.

SIBEITT,'WILMER L.', JR.

$16-6$

ENERGY - ENVIRONMENT SIMULATOR.

MINI-REVIEW. 1978. 4P, (LASL-78-70)

MAXWELL, $G$. BRUCE (E-DO)
FAST - RESPONSE CRYOGENIC

CALORTMETER CONTAINING A

52-KILOGRAM RADIATION ABSORBER.

T1977. 11P. MN (LA-UR-77-515)

ALSO PUBLISHED IN:

ADVANCES IN CRYOGENIC

ENGINEERING, V.23, P.496-502.

Y.D. TIMMERHAUS, ED. PLENUM,

BENDT, PHILIP J. $\left\{\begin{array}{l}\mathrm{P}-2 \\ \mathrm{P}-2\end{array}\right\}$

FI6-8

THE NEVADA TEST SITE

ELECTRO-OPTICS/LASER 77

CONEERENCE AND EXPOSITION.

1977. ANAHEIM, CA PROC.'

P. $545-54$ INDUSTRIAL AND
SCIENTIFIC CONFERENCE MANAGEMENT,

1977 YYONS, PETER B. $(5$ J 35 - 14$)$

GOLOB, JOHN E. (E-3)

LOONEY, LARRY D. $(J-14)$

ROBICHAUD, R. E.

DELSON, M. A.

$16-9$

FIBER OPTIC UTILIZATION AT THE NEVADA TEST SITE.

LASI,

1978. 9P. (LA-7029-MS)

LYONS, PETER B. (J-14)

GOLOB, JOHN E.

LOONEY LARRY D. $(\mathrm{J}-14)$

ROBICHAUD, R. E.

NELSON, M. A.

$16-10$

FIBER-OPTIC TIME DOMAIN

REF LECTOMETER.

TIC 678 . MN (LA-UR-78-824)

LYONS, PETER B. $(\mathrm{J}-14)$

GOLOB, JOHN E. (E-DOR)

NELSON, M. A.

$16-11$

FINAL REPORT ON NRIP-FUNDED MAGNETIC REFRIGERATOR DEVELOPMENT PROJECT, JULY 1, 1975-OCTOBER 1, 1977.

LASL,

1978: 4P. (LA-7111-MS)

STEYERT, WILLIAM A., JR. (Q-10) 
$16-12$

INTERPRETATION OF PULSED EDDY

CURRENT (PEC) IHSPECTION.

1978: 12P. (LA-7459-MS)

LANGNER, GERALD C. $\left(N_{i}-1\right)$

$16-13$

INTERROGATION, AND DETECTION SYSTEM.

PATENTED

FEB 21 1978, FILED 1976.

(U.S.PATENI-4,075,632)

BALDWIN, HOWARD A.
DEPP STEVEN W. (E-DO)
KOELLE ALFRED $(E-3)$
FREYMAR, ROBERT $w .(\mathrm{TD}-7)$

$16-14$

INVESTIGATION OF HEPA FILTERS

SUBJECTED TO TORNADO PRESSURE PULSES INITIAL STRUCTURAL TESTING.

1978. 11P. (LA-7202-MS)

GREGORY, WILLIAM S. (WX-8)

IIORAK, HENRY L. (WX-8)

SMITH' PHIILLIP $R$.

RICKETTS, C. I.

GILL, W.

$16-15$

INVESTIGATION OF HEPA FILTERS

SUBJECTED TO TORNADO PRESSURE

PULSES. PRELIMINARY TESTS OF

INEFFECTIVE. FILTRATION, JULY 1-

SEPTEMBER 30, 1977.

LASL,

HORAK HENRY (LA-7362-PR)

GREGORY, WILLIAM S. $(W X-8)$

SMITH, PHILILIP R.
RICKETTS, C. I.

$16-16$

LOW FLOW VELOCITY, FINE-SCREEN

HEAT EXCHANGERS AND VAPOR - COOLED

CRYOGENIC CURRENT LEADS.

LASL,

1978: 43P. (LA-7395)

STEYERT, h'TLLIAY. A. , JR. (Q-10)

STONE, N. J.

$16-17$

MAGNETIC FEFRICERATOR DEVELOPMENT,

SEPTEMBER 1 - NOVEMBER 30, 1977.

LASL, 5P. (LA-7165-PR)

STEYERT, WILLIAM A., JR. $(Q-10)$ COMP.

$16-18$

MAGIETIC REFRIGERATOK DEVELOPMENT,

MARCH 1-MAY 31, 1978.

LASL, 14P. (LA-7390-PR)

BARCLAY, JOHN A $(Q-10)$
STEYERT, WTLLIAM A., JR. (Q-10)

$16-19$

NEWLY DISCOVERED FAILURE MODE IN HIGH ENERGY DENSITY, ENERGY STORAGE CAPACTITORS.

1978: 5P. (LA-7376-MS)

BOICOURT, GRENEELL P (CTR-11)

KEMP, EDWIN L., JR. (CTR-DO)

$16-20$

NUMERICAL SIMULATIOH OF WIND

FORICES ON STRUCTURES.

197

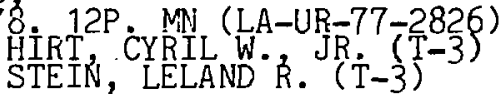

$16-21$

ROTATING CARNOT CYCLE MAGNETIC

REFRIGERATORS FOR USE NEAR $2 \mathrm{~K}$. J. APPL. PHYS.
Sq P $1227-31: 1978$.
STEYERT, WILLIAM A.

JR. $(Q-10$

SIG-22, FAST, PRECISE LENS DESIGN PROCEDURE.

LASL

MINI-REVIEW; 1978. 4P. BRIXNER, BERLYN (Ni-5)

SODIUM VAPOR HEAT PIPE LASER CELL.

TIC 8 . 6P. MN (LA-UR-78-532) DÉVERȦLL, JOIIN E. $(0-13)$

$16-24$

STIRLING CYCLE ROTATING MAGNETIC REFRIGERATCRS AND HEAT ENGINES FOR USE NEAR ROOM TEMPERATURE.

J. A9PL. PIYSS.ंद. 1978. STEYERT, WILLIAM A., JR. (Q-10

$16-25$

TVENT - A COMPUTER PROGRAM FOR ANALYSIS OF TOPNADO - INDUCED TRANSI IN VENTILATION! SYSTEMS.

LASI,' 102P. (LA-7397-M) DUERRE, KENNETH H. $\left(\begin{array}{l}W X-8 \\ \text { ANDRAE, RICHARD } W .\end{array}\right.$

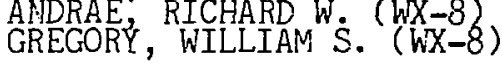

$16-26$

TYPICAL VIDICON RESPONSES TO SHORT DURATION PULSED LIGHT AND FAST

SINGLE - FIELD READOUT.

$$
\begin{aligned}
& \text { LASL, } \\
& 1978 \text { 28P. (LA-7026) } \\
& \text { YATES, GEORGE J J-12) } \\
& \text { HOLMES, VANNER H., JR. (J-12) }
\end{aligned}
$$


$16-27$

UNDERGROUND STRESS ENGINEERING.

THE LIFTING AND STABILIZATION

OF UNDERGROUND VOIDS.

1977

. 27P. MN (LA-UR-77-844)

COLGATE, STIRLING A. (T-DOT)

PETSCHEK, ALBERT G.

BOWERS, MORMAN

$16-28$

VENTILATION SYSTEM PRESSURE

TRANSIENTS. PROPOSED EXPERIMENTS

AND SHOCK TUBE CONCEPTUAL DESIGN

1978. 8P. (LA-7413-MS)

GREGORY, WILLIAM $S .(W X-8)$

SMITH, PHILLIP R. 


\section{7-1}

CONCEPTUAL DESIGN OF AN EMERGENCY

TRITIUM CLEAN-UP SYSTEM.

TIC
1978. 10P. MN (LA-UR-78-1320)
MULLER, MARTIN $(W X-4)$

$17-2$

DOSIMETRY NEEDS FOR THE MAGNETIC

FUSION MATERIALS PROGRAM.

REACTOR DOSIMETRY. 1977 .

PALO ALTO. PROC DOSIMETRY

METHODS FÖR FUELLS CLADDING,

AND STRUCTURAL MATER IALS

P. 1017-30. (NUREG/CP-0004 V.3)

GEINRICH, $\mathrm{R}_{\dot{\mathrm{C}}} \mathrm{R}$.

ODETTE, G. R

FARRAR', HARRY

DIERCKX, R.
GOLD, R.

ENERGY DEVELOPMENT AND THE UPPER

COLORADO RIVER BASIN OPTIMIZATION MODEL.

1978,

MORRIS, GLENN E. (S-2)

$17-4$

GROUIND WATER USE AND FOSSIL FUEL

CONVERSION.

1978: 6P. (LA-7492-P)

LOOSE, VERNE W. (S-2) 
18. ENVIRONMENT CONTROL TECHNOLOGY AND EARTH SCIENCES

18-1

ATMOSPHERIC PARTICULATE SAMPLING

PROCEDURE FOR GRAVIMETRIC AND

ELEMENTAL ANALYSES.

J. AIR POLLUT. CONTROL ASSOC., WAMGEN, LAWRENCE E. (H-8)

$18-2$

CENOZOIC VOLCANIC GEOLOGY AND PROBABLE

ACE OF INCEPTION OF BASIN - RANGE

FAULTING IN THE SOUTHEASTERNMOST

CHOCOLATE MOUNTAINS, CALIFORNIA.

GEOL. SOC. AM. BULLL.

CROWE, BRUCE M. $(\dot{Q}-21)$

COMPARE-MOD 1. A CODE FOR THE

TRANSIEHT ANALYSTS OF VOLUMES WITH

HEAT SINKS, FLOWING VENTS, AND

DOORS.

1978

$978,66 \mathrm{P}$

GIDO, RICHARD G.: $(Q-6)$

LAWTON,' ROBERT G. $(\bar{G}-3)$

JENSEN, WALTON L.

$18-4$

DEVELOPMENT OF HIGH - TEMPERATURE

ACOUSTIC INISTRUMENTATION FOR

CHARACTERIZATTON OF HYDRAULIC

FRACTURES IN DRY HOT ROCK.

INSTRUMENT SOCIETY OF AMERICA.

INTERNATIONAL INSTRUMEMTATION SYMP.

$1976(N A Y)$. 2 2ND SAI DIEGO.

PREPRINT. ISA ASI $76222(97-108)$

ISA, 1976

DENAIS; BERT R. (Q-22)

HIILL, JAMES $H .(Q-22)$

STEPHANI, EVON $(Q-22)-22)$

TODD, BILLY E. $(\dot{Q}-2 \dot{2})$

DISPLACEMENTS AND STRESSES ABOUT

AN ARBITRARILY LOADED SPHEROIDAL

INCLUSION.

$$
\begin{aligned}
& \text { LASL, } 36 P, \text { (LA-7035-MS) } \\
& \text { 1978 } 36 \text { SHER, ROBERT } \\
& \text { WARREN, NICHOLAS W. (Q-21) }
\end{aligned}
$$

$18-6$

ELECTRICAL CONDUCTIVITY AND

VELOCITY ANOMALIES IN PARTIAL MELTS.

KIMBERLITE CONFERENCE. 1977.

2ND INIERNATIONAL, SANTA FE; MM.

EXTENDED ABSTRACTS, 2 PAGES.

THE CONEERENCE, 1977.

(549/K 49I 1977 A)

SHIANKIAND, THOMAS J. (G-6)
18-7

ESTIMATION OF EXPECTED VALUE AND COEFFICIENT OF VARIATION FOR LOGNOR AND GAMMA DISTRIBUTIONS. LASL, 82P. (LA-7393-MS)
1978. WHIT, GARY C. (H-12)

$18-8$
EXCAVATIONS ON MESITA DE LOS ALAMOS LASL, 17P. (LA-7043-MS) WORMAN, FREDERICK C. V.
STEEN, CHARLIE R.

18-9

EXPERIMENTAL PERMEABILITY STUDIES

AT ELEVATED TEMPERATURE AND

PRESSURE OF GRANITIC ROCKS.

LASL,

1978: 101P. THESIS, STANFORD

UNIV: 1978. (LA-7224-T)
POTTER, JARED MICHAEL

$18-10$

FIELD PROCEDURES FOR THE URANIUM HYDROGEOCHEMICAL AND STREAM SEDIMEN RECONNAISSANCE AS USED BY THE LOS ALAMOS SCIENTIF IC LABCRATORY.

LASL, 64P. (LA-07054-M) AHARP ROBERT R. (GR;

$18-11$

FURTHER STUDIES OF LONG - TERM ECOLOGICAL EFFECTS OF EXPOSURE TO URANIUM.

LASL, 27P. (LA-07162)

$$
\begin{aligned}
& \text { HANSON, WAYNE C. (H-12) } \\
& \text { MIERA, FELIX R., JR. (H-12) }
\end{aligned}
$$

18-12

GERMANIUM DETECTOR SYSTEM FOR

THE DETECTION OF TRANSURANICS AT LOI ACTIVITY CONCENTRATIONS IN SOIL.

TIC

$$
\begin{aligned}
& \text { 13P. MN (LA-UR-78-87) } \\
& \text { WEST, LEON, JR }(\mathrm{H}-1) \\
& \text { UMBARGER, C. JOHN }(H-1) \\
& \text { DEMPSEY, THOMAS }
\end{aligned}
$$

18-13

GRAVITY SURVEY OF THE PAJARITO

PLATEAU LOS ALAMOS AND SANTA FE COUNTIES, NEW MEXICO.

LASL,

1978. 15P. (LA-7419-MS) 
$18-14$

GROSS ALPHA ACTIVITY AS AN

ESTIMATOR OF RADIUM -226 ACTIVITY

IN SOILS AND TAILINGS AT AN

INACTIVE URANIUM MILL TAILINGS

SITE

$$
\begin{aligned}
& \text { LASL, 6P. (LA-7529-MS) } \\
& 1978 \text { REESEN, DAVID R. (H-12) } \\
& \text { WIENKE, CAROLINE (H-8) }
\end{aligned}
$$

$18-15$

HEAVY METHANES (MASS -20 AND -21)

AS ATMOSPHER IC TRACERS - A NEW

RESEARCH INITIATIVES PRCJECT.

$$
\text { LASL, 93P. (LA-7321-MS) }
$$

FOWLER, MALCOLM M. (CNC-11) COMP. COWAN, GEORGE A. (CNC-DO)

BARR, SUMNER $(G-8)$

CLEMENTS, WILLIAM E. $(G-3)$

18-16

HYDROGEOCHEMICAL AND STREAM SEDIMENT

RECONNAISSANCE OF THE NATIONAL URANIUM

RESOURCE EVALUATION PROGRAM, APRIL-

JUNE 1977 THE ROCKY MOUNTA IN STATES

OF NEW MEXICO COLORADO, WYOMING, AND

MONTANA, AND THE STATE OF ALASKA:

LASL,

1978: 28P. (LA-6992-PR)

BROXTON, DAVID E $(G-5)$

18-17 17 OOCOCHEMICAL AND STREAM SEDIMENT

RECONNAISSANCE OF THE NATIONAL URANIUM

RESOURCE EVALUATION FROGRAM JULY-

SEPTEMBER 1977. THE ROCKY MOUNTA.IN

STATES OF NEW MEXICO, COLORADO

WYOMING, AND MONTANA; AND THE STATE OF

ALASKA

LASL,

978. 14P. (LA-6993-PR)

NUNES, HENRY PAUL $(G-5)$

$18-18$

HYDROGEOCHEMICAL AND STREAM SEDIMENT RECONNAISSANCE OF THE NATIONAL

URANIUM RESOURCE EVALUATION PROGRAM.

LASL

1978: 10P. (LA-7175-PR)

MORRIS, WAYNE A. $(J-5)$

BUNKER, MERLE E. (P-2)

18-19

INFLUENCE OF ACCESS HOLE PARAMETERS

ON NEUTRON MOISTURE PROBE READINGS.

LASL

ABEELE, WILLY V. (H-8)
$18-20$

INVESTIGATIONS OF THE NATURAL FISSICN

REACTOR PROGRAM, OCTOBER, 1977 -

SEPTEMEER, 1978.

LASL,

1978 24P. (LA-7536-PR) NORAIS, ANEREW E. (DIR OFF) ED.

MIG-21 PRODUCTS AND APPLICATIONS TC THE NUCLEAR WASIES.

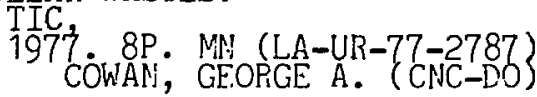

$18-22$

NORTHWEST NEW MEXICO LOUNDARY LAYER

EXPERTMENT.

LASL

ARCHULETA JOAN A- J

BARR, SUMRER (G-8)

CARLOS ROBERT $\mathrm{C}$. $(\mathrm{J}-10)$

CLEMENTS, WILLIAM E. (G-8)

LABRY, JOHN $(\mathrm{H}-8)$

WANGEN, LAWRENCE E. (CNB-8)

WILSON', SAMUEL K. (H-8)

$18-23$

NUMERICAL SIMULATION OF A VERY

SURFACE WITH POSSIBLE

APPLICATION TO TEKTITES.

IMPACT AND EXPLOSION

CRATERING. D.J. ROLDY, ED.

P. $1009-24$ PERGAMON, 1977 .

JÖNES, ERIC M. ( $\mathrm{J}-9)$

$(\mathrm{J}-\mathrm{g})$

$18-24$

PARTIAL MELT IN REGIONS OF

HIGH ELECTRICAL CONDUCTIVITY.

PARTIAL MELTING IN THE EARTH'S

UPPER MANTLE 1976. AMERICAN

GEOPHYSICAL UNNION, CHAPNAN

CONFERENCE, BROOKINGS, OR

AND THE JOSEPHI ${ }^{\prime}$ E PERIDCTITE.

PROC., MAGMA GENESIS,

P.215-8. OREGON DEPT' OF

GEOLOGY AND NINERAL

INDUSTRIES, 1977 . (551.1/P273M)
SHANKLARD, THOMAS J. (Q-21) 
$18-25$

PLUTONIUM AMERICIUM AND URANIUM IN BLOW-SAND MOUNDS OF SAFETY-SHOT SIIES AT THE NEVADA TEST SITE AND THE TONOPAH TEST RANGE. TIC ESSINGTON EDWARD H. (H-8) GILEERT, RICHAFD 0 . WIREMAN, D L. FOWLER, ERIC B. $(H-8)$

$18-26$

POSSIBLE URANIUM - RUTHENIUM

METHOD FOR MEASUREMENT OF

ORE AGE.

TIC.7 7P. MN (LA-UR-77-2863)

APT KENNETH E. (CNC-DO)
COWAN, GECRGE A. (CNC-DO)

$18-27$

PRELIMTNARY DESIGN OF A PROTOTYPE

PARTICULATE STACK SAMPLER.

1978 ,

ELDER, JOHN C ( $\left.\mathrm{H}^{-5}\right)$
LITTLEFIELD LARRY $(\mathrm{H}-5)$
TILLERY, MIARVIN I.
ETTINGER, HARRY J. $(H-5-\mathrm{T})$

$18-28$

PRELIMINARY OPTIMIZATION MODEL FOR ASSESSMENT OF ENERGY DEVELOPMENT IN

THE ROCKY MOUNTAIN RECION.

LASL

1078: 58P. (LA-6689-MS)

HINNAN, GEORGE W.

KOLSTAD, CHARLES D. $(Q-12)$

MORRIS, GLENN E. $(\dot{Q}-12)$

PALMER, RONALD G $(Q-12)$
PENDLEY, ROBERT $(2-12)$

$18-29$

PRELIMINARY PLANE TABLE MAP, SODA DAM, JEMEZ SPRINGS, NM' 1978.' (LA-7520-MAP)

GOFF, FRASER E. $(G-6)$

STEWART, BRUCE S.

$18-30$

RAW DATA REPORT OF ELEMENTAL ANALYSES

FROM HYDROGEOCHEMICAL AND STREAM

SEDIMENT SANPLES TAKEN NEAR STERLING

AND FORT MORGAN, NORTHEASTERN COLORADO,

DECEMBER 1976 AND JANUARY 1977.

LASL

1977 . 27P. (LA-6740-MS)
18-31

RECENT DEVELOPMENTS FOR FIELD

MONITORING OF ALPHA - EMITTING

CONTAMINANTS IN THE ENVIRONMENT.

HEALTH PHYS.

V.34, P. 486-9. 1978.

AHILQUIST, A. JOHN $(\mathrm{H}-8)$

UMEARGER C C: JOHN $\left(\mathrm{H}^{-1}\right)$

$18-32$

RESIDUAL BOUGUER GRAVITY ANOMALY MAP OF NEW MEXICO.

1978: (LA-07466-MAP)

AIKEN, CARLOS L. V.
LAUGHLIN A. WILIIAM (G-9)
WEST, FRANCIS G. (G-9)

$18-33$

SCALING OF CRATERING EXPERIMENTS AN ANALYTICAL AND HEURISTIC

APPROACH TO THE PHENOMENOLOGY.

PLANETARY CRATERING

MECHANICS. 1976 SYMPOSIUM,
FLAGSTAFF; A2. PROC IMPACI

AND EXPLOSION CRATERING PLANETARY

AND TERRESTRIAL IMPLICATIONS. D.J.

RODDY, ED P $1165-90$. PERGAMON,

KILLIAN, BARBARA GERMAIN $(\mathrm{J}-9)$
GERMAIN, LAWRENCE S. (J-DOT)

$18-34$

SEARCH FOR OTHER NATURAL FISSION REACTORS.

TIC.7. 15P. MN (LA-UR-77-2796)

APT KENNETH E. (CNC-DO)

BALAGNA, JOHN P. JR. (CNC-11)

BRYANT, ERNEST $\dot{A}$ ' (CNC-11)

COWAN, GEORGE A. (CNC-DO)

DANIELS, W'ILLIAM R. $(C N C-11\}$

18-35 IN INUESTIGATION OF THE NUCLEAR FUEL SERVICES, INC , REPROCESSING PLANT AT WEST' VALLEY, NEW YORK.

LASL

END 33P (LA-7087-MS)

BARTHOLOMÉW, ROBERT J. $(W X-8)$

BENNETT, JOEL G. $(Q-13)$

BRASIER, ROBERT I

CORCORAN, WILLIAM F. (WX-8)

SOIL 36 SURVEY OF LOS ALAMOS COUNTY, NEW NEXICO.

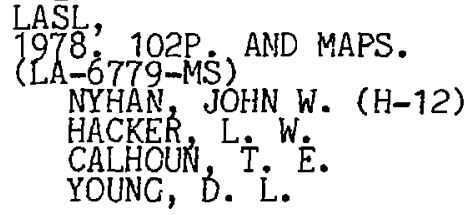

LASL

$1978,102 P$. AND MAPS. NYHAN, JOHN W. $(\mathrm{H}-12)$

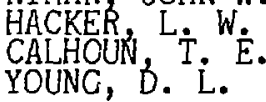


18-37

SOME ATMOSPHERIC TRACER EXPERIMENTS

IN COMPLEX TERRA IN AT LASL

EXPERIMENTAL DESIGN AND DATA.

LASL,

1978' 38P. (LA-7198-MS VOL. 1)
ARCHULETA JOHN A (H-8)
BARR, SUMNER (G-8)
CLEMENTS, WILLIAM E. (G-8)
GEDAYLOO; TEYMOOR $(H-8)$
WILSON, SAMUEL K. (H-3)

$18-38$

SORPTION - DESORPTION STUDIES OF NEVADA TEST SITE ALLUVIUM AND DEBRIS.

LASL

1978 32P, (LA-7216-MS)

STATISTICAL THEORY OF FRAGMENTATION. TIC
DIENES, MOHN K. $(\mathrm{T}-3)$

$18-40$

SUMMARY OF THE SEISMIC ANALYSES OF THE NUCLEAR FUEL SERVICES REPROCESSING

PLANT AT WEST VALLEY, NEW YORK. LASL, ENDEBRȮCK, ELTION G. $(W X-8)$

$18-41$

TEMPORAL CHANGES IN THE DISTRIBUTION

OF CESIUM-137 IN ALLUVIAL SOILS AT

LOS ALAMOS.

LASL,

$$
\begin{aligned}
& \text { 78: } 11 P .(L A-7298-M S) \\
& \text { NYHAN JOHN W. }(H-8) \\
& \text { HAKONSON, THOMAS E. }(H-8) \\
& \text { MIERA FELIX R. JR. }(H-12) \\
& \text { BOSTICK, KENNETH V. }(H-8)
\end{aligned}
$$

$18-42$

TILTS ASSOCIATED WITH VOLCANIC

ACTIVITY, GUADELOUPE, FRENCH WEST INDIES, FALL 1976.

$$
\begin{aligned}
& \text { LASL, 10P, (LA-7500-MS) } \\
& \text { 1978. WEST, FRA NCIS G. (G-9) } \\
& \text { HEIKEN, GRANT (G-6) } \\
& \text { HOMUTH, EMIL F (G-2) } \\
& \text { PETERSON ROBERT W. }(\mathrm{J}-14) \\
& \text { CROWE, BRUCE M. (G-6) }(\mathrm{G}-6) \\
& \text { WOHLETZ, KENNETH H. (G-6) }
\end{aligned}
$$

\section{$18-43$}

TWO NEW PORTABLE SURVEY

INSTRUMENTS. THE FIELD PHOSWICH DETECTOR AND THE WEE PEE WEE.

\section{TIC,}

$18-44$

URANIUM CONCENTRATIONS IN STREAM

WATERS AND SEDTMENTS FROM

SELECTED SITES IN THE EASTERN

CHARLEY RIVER AREAS, AND ACROSS

SOUTH - CENTRAL ALASKA.

1978. 39P. AND PLATES.

(LA-6649-MS)

$$
\text { SHAKP, ROBERT R. (GR; }
$$

$18-45$

URANIUM HYDROGEOCHEMICAL AND

STREÁY SEDIMENT RECONNAISSANCE

DATA FROM THE AFEA OF THE TELLER, BENDELEBEN, CANDLE AND KATEEL RIVER QUADRANGLES, SEWARD PENINSULA AND VICINITY, ALASKA.

$$
\begin{aligned}
& \text { LASL, } \\
& \text { 197819. 161P. AND PLATES. } \\
& \text { (LA-6844-MS ) } \\
& \text { SHAPP, ROBERT R. (GR. (G-5) } \\
& \text { HILL, DWIGHT E. (G-5) }
\end{aligned}
$$

$18-46$

URANTUM FYDROGEOCHEMICAL AND

STREAM SEDIMENT RECONNA ISSANCE DATA FROM THE AREA OF THE SFYSHMAREF KOTZEBUE, SELAWIK AHD SHUNGHAK QUADRANGLES MORTHERM SENARD PENINSULA A ND VICINITY, ALASKA.

LASL,

1978: 94P. AND PLATES.

LA-6845-MS)

WARREN, KICHARD G. $(G-5)$

HILL, DWIGHT E. $(\dot{G}-5)$ (G-5)

$18-47$

URANIUM HYDROGEOCHEMICAL AISD

STREAM SEDIMENT RECONNA ISSANCE OF THE DENVER AND GREELEY NTMS QUADRANGLES, COLORADO.

$$
\begin{aligned}
& \begin{array}{l}
\text { LASL, } 138 \mathrm{P} . \\
(19 \mathrm{~g}-7177-\mathrm{MS})
\end{array} \\
& \text { BOLIVAR, STEPHEN L. }(G-5) \\
& \text { BROXTON' DAVID } \frac{E}{\text { OLSEN, }}\left(\begin{array}{l}
G-5 \\
G-5
\end{array}\right)
\end{aligned}
$$

$18-48$

URANIUM HYOROGEOCHEMICAL AND STREAM SEDIMENT RECONNA ISSANCE OF THE RAW'INS NTMS GUADRANGLE, WYOMING.

$$
\begin{aligned}
& \text { LASL, } \\
& 1978.73 P \text { P AND PLATES. } \\
& \text { (LA-7179-MS) } \\
& \text { WEAVER, THOMAS A. }(G-5) \\
& \text { MORRIS; WAYNE A. }(G-5) \\
& \text { TREXLER, PAMELA K. }(G-5)
\end{aligned}
$$


18-49

$\because$ RANIUM HYDROGEOCHEMICAL AND TREAM SEDIMENT RECONNA ISSANCE DATA RELEASE FOR THE NEW MEXICO PORTIONS OF THE HOBBS AND BROWNE IELD NTMS QUADRANGLES, NEW NEXICO / TEXAS.

ASL

1978: 61P AND PLATES.

(LA-7182-MS)

WARREN, RICHARD G. $(G-5)$

NUNES, HENRY PAUL $(G-5)$

$18-50$

URANIUM HYDROCEOCHEMICAL AND

STREAM SEDIMENT RECONNAISSANCE

OF THE TULAROSA NTMS QUADRANGLE, NEW MEXICO.

LASL, $25 \mathrm{P}$ AND PLATES.
(LA-7217-MS)

BROXTON, DAVID E. $(\mathrm{G}-5)$

$18-51$

URANIUM HYDROGEOCHEMICAL AND

STREAM SEDIMENT RECONNA ISSANCE

DATA RELEASE FOR THE STERLING

NTMS QUADRANGLE, COLORADO.
LASL
1978 . 57P AND PLATES.
NUNES, HENRY PAUL (G-5)

$18-52$

URANIUM HYDROGEOCHEMICAL AND STREAM SEDIMENT RECONNAISSANCE OF THE NORTHERN PART OF THE POWDER RIVER BASIN, WYOMING.

LASL,

1977? 80P. (LA-6654-MS) MORRIS; WAYNE A. (J-5)

$18-53$

URANIUM HYDROGEOCHEMICAL AND STREAM SEDIMENT RECONNAISSANCE DATA FROM THE AREA OF THE NOATAK AND PORTIONS OF THE BAIRD MOUNTAINS AND AMELER RIVER QUADRANCLES, ALASKA.

(2A-6846-MS)

SHLLA DWIGHT E. $(G-5)(G-5)$

$18-54$

URANIUM FYYDROGEOCHEMICAL AND STREAM SEDIMENT RECONNAISSANCE IN

SOUTHNESTERN MONTANA.

LASL

1978: 95P. AND MAPS.

BROXTON, DAVID E. $(\mathrm{G}-5)$
$18-55$

URANIUM HYDROGEOCHEMICAL AND STREAM

SEDIMENT RECONNA ISSANCE OF THE

EKALAKA NTMS QUADRANGLE

MONTANA.

LASL

1978: 71P. (LA-7069-MS)

CHANEY, DAN S. $(\mathrm{G}-5)(\mathrm{G}-5)$

$18-56$

URANIUM HYDROGEOCHEMICAL AND STREAM SEDIMENT PILOT STUDY OF THE BOULDER BATHOLITH, MONTANA.

LASL

1978 : $118 \mathrm{P}$. (LA-7 176-MS)
AAMODT, PAUL L. (G-5)

$18-57$

URANIUM HYDROGEOCHEMICAL AND STREAM

SEDIMENT RECONNAISSANCE DATA

RELEASE FOR THE MONTANA PORTION

OF THE HAMILTON, MONTANA/IDAHO,

NTMS QUADRANGLE.

LASL, 45P. (LA-7178-MS)
AAMODT; PAUL L. (G-5)

$18-58$

URANIUM HYDROGECCHEMICAL AND STREAM SEDIMENT RECONNA ISSANCE DATA RELEASE OF THE DOUGLAS, SILVER CITY CLIFTON, AND SAINT JOHNS NTMS QUADRAKGLES, NEW MEXICO/ARIZONA.

1978: 123P. (LA-07180-MS)

SHARP ROBERT R., JR. (G-5)

MORRIS', WAYNE

AAMODT, PAUL L. (G-5)

$18-59$

URANIUM HYDROGEOCHEMICAL AND STREAM

SEDIMENT RECONNA ISSANCE DATA RELEASE FC THE WYOMING PORTIONS OF THE DRIGGS PRESTON, AND OGDEN NTMS QUADRANGLES.

LASL

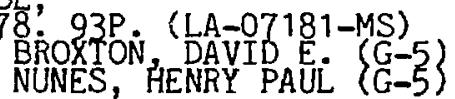

$18-60$

URANIUM HYDROGEOCHEMICAL AND STREAM SEDIMENT RECONNA ISSANCE OF THE PUEBLO NTMS QUADRANGLE, COLOPADO

LASi,

$1978,77 P$ AND PLATES. LA-7341-MS) SHANNON, SPENCER S., JR. (G-5) 
$18-61$

URANIUM HYDROGEOCHEMICAL AND STREAM

SEDIMENT RECONNA ISSANCE OF THE RATON

NTMS QUADRRANGLE, NEW MEXICO.

1978: 84P. AND PLATES.

(LA-7344-MS)

MORGAN, TERRANCE L, $(G-5)$
BROXTON, LAVID E. $(G-5)^{-5}$

$18-62$

URANIUM HYDROGEOCHEMICAL AND STREAM

SEDIMENT RECONNAISSANCE OF THE

TRINIDAD NTMS QUADRANGLE, COLORADO.

1978. 79P. AND PLATES.

(LA-07345-MS)

MORRIS, WAYNE A. (G-5)

LA DELFE CAROL M. $(G-5)$

$18-63$

URANIUM HYDROGEOCHEMICAL STREAM

SEDIMENT RECONNAISSANCE OF THE

AZTEC NTMS QUADRANGLE,

NEW MEXICO.

1978: 75P AND PLATES.

(LA-7238-MS)

BOLIVAR, STEPHEN L. $(G-5)$

$18-64$
URANIUM MILL TAILINGS - ENVIRONMENTAL

IMPLICATIONS.

MASL, REVIEW, 1978. 4P.

(LASL-77-37) DAVID R. (H-8)

$18-65$

URANTUM SOLUBILITIES IN THE

OKLO REACTOR ZONES.

TIC73.9P. MN (LA-UR-77-2218)

DUFFY; CLARENCE J. (CNC-11)

$18-66$

VISIBILITY ISSUE IN THE ROCKY MOUNTAIN

WEST

$\mathrm{TIC}_{2}$

LEONAPD MN (LA-UR-77-2558)

WILLTAISS MICHAEL D

MUTSCHLECNER, J. PAUL

$18-67$

VORP

SYSTEM FOR REMOTE MEASUREMENTS.

1978. 18P. MN (LA-UR-78-26)

SMALE RICHARD F. (H-1)

BACKSEN, MICHAEL H. $(E-5)$
$18-68$

WATER QUALITY IN THE VICINITY OF FENTON HILL SITE, 1977. LASL

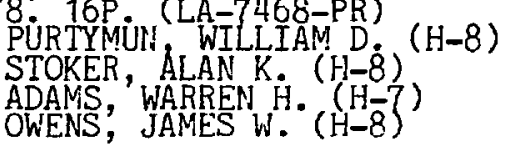

$18-69$

WATER QUALITY IN VICINITY OF

EENTON HILL SITE, 1976.

1978. 17P. (LA-7307-MS)

ADAMS, WARREN $\mathrm{H}$. ( $\mathrm{H}-7)$

STOKER, ALAN K. $(\mathrm{H}-8)$

WATER SUPPLY AT LOS ALAMOS

DURING 1977.

LASL, 38P. (LA-7436-MS)

PURTYMUN, WILLIAM D. (H-8)

$18-71$

1978 INTERNATIONAL SYMPOSIUM ON THE RIO GRANDE RIFT, OCTOBER 8-17, 1978, AND ABSTRACTS.

LASL, 1058 (LA-7487-C)
OLLSEN, KENNETH H. (G-2)
CHAPIN, CHARLES E.

$\mathrm{CH} A$ 
19. EXPERIMENTAL PLASMA PHYSICS

$19-1$

ABSOLUTE RADIATION MEASUREMENT AND

COMPARISON WITH TOTAL ENERGY LOSS. HIGH-TEMPERATURE PLASMA DTAGNOSTICS. 1978 2 ND TOPICAL CONE SANTA EE, ABSTRACTS, P.90.
HOWELL ROBERT B: (CTR-2)
HABERSTICH, ALBERT (CTR-2)
KARR, HUGH'J. (CTR-2)

$19-2$

AUTOMATED COMPUTER ANALYSIS OF PLASMA STREAK TRACES FROM SCYLLAC.

TIC,
1977. 22P, MN (LA-UR-77-2582)
WHITMAN, ROLLIN L $(N-8)$
JAHOCA, FRANZ C. (CTR-8
KRUGER, RICHARD (N-8)

JAHOCA, 'FRANZ C. (CTR-8
KRUGER', RICHARD ( $(.-8)$

$19-3$

COLIECTIVE ION ACCELERATION, JULY $1976-$ SEPTEMBER 1977.

LASL, 281P. (LA-7148-PR)

GODFFEY; EKENDAN B; $(T-15)$

FAEHL, $R . J .(T-15)$

NWWEERGER, EARPY S. (T-15)

THODE, LESTER E. $(T-15)$

$19-4$

COMFUTER ANALYSIS OF 2-D

HOLOGRAPHIC INTEREEROCRAMS.

HIGH-TEVYPERATURE PLASIAA DIAGNOSTICS.

1978 2ND TOPICAL CONF SANTA EE,

CONEAD, ROSEMARY (CTR-8)

DESICN CONSIDERATIONS FOR THE AIR

FORCE WEAPONS LABORATCRY FX25

PLASMA HEATING EXPERIMENT.

LASL,
T97. 9P. (LA-7233-MS)
THODE, LESTER E. (T-15)

$19-6$

DIAGNOSTIC FOR MEASUREMENT OF

RADIATED ENERGY FORM A TOROIDAL PLASMA.

$$
\begin{aligned}
& \text { LASL, 11P. (LA-7273) } \\
& 1978 \text {. TWELL, ROBERT } \\
& \text { HOW- (CTR-2) } \\
& \text { KARK, HUGH J. }
\end{aligned}
$$

19-7

WETERODYNE INTERFEROMETRY.

HIGH-TEMPERATURE PLASMA DIAGNOSTICS.

1978. 2ND TOPICAL CONF SANTA FE,

NM. ABSTRACTS P. 48 . (LA-71)
KRISTAL, RICHARD (CTR-8)
19-8

HETERODYNE QUADRATURE INTERFEROMETE

FOR SIMULTANEOUS MEASUREMENTS OF PL

DENSITY ALONG SEVERAL CHORDS.

REV SCI. TNSTRUM. JACCBSON, ABBRAM Rि. (CTR-2)

$19-9$

LASER - PLASMA INTERACTION IN A THETA - PINCH GEOMETRY.

LAST, 152P. THESIS UNIV. OF COLORADO 1978 (LA-7302-T)
ARMSTRONG, WILLIAM T. (CTR-11

$19-10$

MEASUREMENT OF LINE DENSITY WITH

A MULTI-PASS INTEREEROMETER. HIGH-TEMPERATURE PLASMA DIAGNOST 1978. 2ND TOPICAL CONE. SANTA F NM. ABSTRACTS, P.61 (LA-7 160-C)
ARMSTRONG, WILLIAM T. (CTR-3) MILLER, GUTHRIE (CTR-3)

$19-11$

MEASUREMENTS OF THE PLASMA ABLATED FROM AN END PLUG IN A HIGH ENERGY THETA PINCH USING RUBY LASER SIDE VTEWING HOLOGRAPHIC INTERFEROMETRY

HIGH-TEMPERATURE PLASMA DIAGNOST 1978. 2ND TOPICAL CONF. SANTA F NM. ABSTRACTS, P. 60. (LA-7160-C) EKDAHL CARL A. JR. (CTR-3) COMMISSO, ROBERT J. (CTR-3) MC KENNA, KENNETH $F$, (CTR-3) SIEMON, RICHARD E. (CTR-3)

19-12

ON PLASMA TRANSPORT AND FLOW IN THE ELUID PICTURE.

LASL, 19P. (LA-7429-MS) MILLER, GUTHRIE (CTR-3)

$19-13$

PRELIMINARY INVESTIGATION OF ANOMALOUS RELATIVISTIC ELECTRON BEAM DEPOSITION INTO A 10(17) TO $10(20)$ CM-3 DENSITY PLASMA. LASL, 21P. (LA-7215-MS) THODE, LESTER E. (T-15) 
$19-14$

SCYLLA IV-P COMPUTER BASED CONTROL AND

DATA ACOUISITION SYSTEM

ENGINEER ING PROBLEMS OF FUSION

RESEARCH. 1977. SYMP. $7 T H$,

KNOXVILIE, TN. PROC, V.2,

P. 1213-6. M. S. LUBELLE ED

ALSO'PUBLISHED IN :

TIC

1977 . 5P. MN (LA-UR-77-2405)

CONRAD, ROSEMARY (CTR-8)

LILLBERG, JOHN W. (CTR-8)
WILKINS, RONALD $w$. (CTR-8)

19-15

SECOND TOPICAL CONFERENSE ON HIGH

TEMPERATURE PLASMA DIAGNOSTICS, $19 \overline{7} 8$

ABSTRACTS:

LASL

1978: 115P. (LA-7160-C)

JAHODA, FRANZ C. (CTR-8) COMP.

$19-16$
SUMMARY OF SCYLLAC RESULTS.

1978, 27P. (LA-7125-MS)

SIEMON, RICHARD E. (CTR-3)

19-17

TEMPERATURE DIAGNOSTICS USING

LI-LIKE SATELLITES.

HIGH-TEMPERATURE PLASMA DIAGNOSTICS.

1978. 2ND TOPICAL CONF SANTA FE,

JONES, LARRY A. $(P-3)$
THOMSON, DAVID $3 .(P-3)$

19-18

TESTING TRANSIENT RECORDER LINEARTTY

HIGH-TEMPERATURE PLASMA DIAGNOSTICS.

1978. 2ND TOPICAL CONF.

NM. ABSTRACTS, .67 (LA-7160-C)

KLARE, KENNETH ${ }^{\circ}$ : $\left(\begin{array}{l}\text { CTR-8 } \\ \text { KL }\end{array}\right.$ 
20. FUSION SYSTEMS

20-1

AIR DRIVEN FIEER OPTIC COUPLED

PULSER SYSTEM FOR ZT-40.

ENGINEERING PROBLEMS OF FUSION

RESEARCH. 1977. SYMP. 7TH,

KNOXVILLE, TN. PROC., V. 1,

P.415-7. M.S. LUBELL, ED.

IEEE, 1977. (537/S989EN 1977)

ALSO PUBLISHED IN:

T977. 4P. MN (LA-UR-77-2407)

NUNNALLY, WILLTAM C. $($ CTR-11)

$20-2$

ANALYSIS OF A DC COMMUTATOR

MACHINE FOR EXCHANGE OF ENERGY

WITH A SUPERCONDUCTING COIL.

LASL,

1978: 14P. (LA-7447-MS)

VOGEL, HERBERT F. (CTR-9)

$20-3$

CIRCUIT AND PLASMA SIMULATION

FOR THE DESIGN OF ZT-40.

ENGINEER ING FROBLEMS OF FUSION

RESEARCH. 1977. SYMP. 7TH,

P.501-5. M. S. LUBELL' ED.

IEEE, 1977 (537/S989́EN 1977)

ALSO PUBLISHED IN:

1977 5P. MN (LA-UR-77-2333)

BOICOUUR, GRENFELL P. (CTR-11)

$20-4$

CONCEPTUAL DESIGN OF AN EMERGENCY

TRITIUM CLEAN - UP SYSTEM.

1978, 9P. AND APPEND.

(LA-7136-MS)

COST-CONSTRAINED DESIGN POINT

FOR THE REVERSED-FIELD PINCH

REACTOR (RFPR).

T978̊. 12P. MN (LA-UR-7 -1397 )

HAGENSON, RANDY L. (CTR-12)
KRAKOWSKI, ROBERT A. (CTR-12)

$20-6$

CURRENT CONTROL BY A HOMOPOLAR MACHINE WITH MOVING BRUSHES.

LASL, 8P, (LA-7090-MS)
VOGEL; HERBERT F. (CTR-9)
20-7

DESIGN AND FABRICATION OF A RADIALL

FED IMPLOSION HEATING COIL.

ENGINEERING PROBLEMS OF FUSION

RESEARCH, 1977 . SYMP. 7TH,

P.830-3. M.S. LUBELL, ED.

IEEE, T977 (537/S989EN 1977)

ALSO PUBLISHED IH:

${ }_{197}$. 5P. MN (LA-UR-77-2452)

HANSBOROUGH, LASH D. (MP-14)

DICKINSON JAMES $M$. (CMB-6)

MELTON, JIMMY G. $(C T R-11)$

NUNNALIY, WILLIAM C. (CTR-11)

20-8

DEVELOPMENT OF A LOW - COST, 10-KV

CAPACITOR

ENGINEERING PROBLEMS OF FUSION

RESEARCH. 1977. SYMP. 7TH,

KNOXVILLE, TN. PROC V V.2,

P. 1757-9. M. S. LUBELL ED.

IEEE' 1977 .

TIC. 4P. MN (LA-UR-77-2408)

KEMP EDWIN L JN J (CTR-DO)
BOICOURT, GRENFELL (CTR-11)

BICKFORD; K. JAMES (CTR-4)

$20-9$

DEVELOPMENT OF THE SWITCHINC COMPONENTS FOR ZT-40.

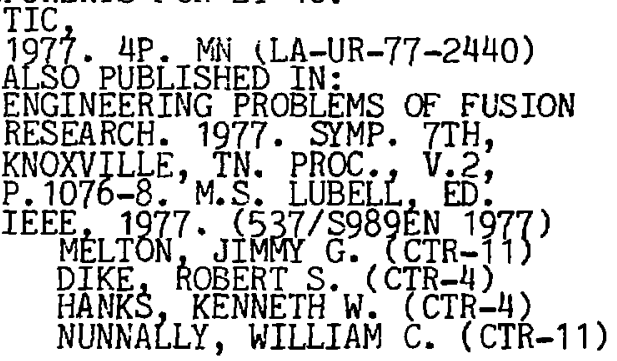

20-10

ENGINEERING DESCRIPTION OF THE LASL
ZT-4O TOROIDAL Z-PINCH.

ENGINEERING PROBLEMS OF FUSION

RESEARCH. 1977. SYMP. 7TH,

KNOXVILLE TN. PROC, V.1,

P.655-8 M. S, LUBELL ED 977 )

ALSO'PUBLISHED IN:

TIC

$$
\begin{aligned}
& \text { HAMMPER MN CHARLESR-77-2441) } \\
& \text { DIKE ROBERT S. (CTR-4) } \\
& \text { NUNNALLY, WILLIAM C. (CTR-11) }
\end{aligned}
$$


20-11

FEEDBACK STABILIZATION OF A

LAMBDA EQUALS $0,1,2$ HIGH BETA

STELLARATOR

NUCL. FUSION,

18 . $1027-49.1978$.

BARTSCH, R. R. (CTR-3)

CANTRELL, EMMETT L. (CTR-3)

GRIBBLE, ROBERT F * (CTR-3)

KLARE, KENNETH A. $($ CTR-8

KUTAC, KENNETH J (CTR-4)

QUINN, WARREN E. (CTR-DO)

$20-12$

FEEDBACK STABILIZATION OF AN L $=0,1,2$ HIGH - BETA STELLARATOR.

LASL,

1978? 28P (LA-7243)

BARTSCH, R. R. (CTR-3)

CANTRELL, EMMETT L. (CTR-3)

GRIBBLE, ROBERT F. (CTR-3)

KLARE, KENNETH A. (CTR-8)

KUTAC, KENNETH J (CTR-4

MILLER, GUTHRIE (CTR-3)

20- 13

FUELINGG BY COAXIAL PLASMA GUNS.

1977. 5P. MN (LA-UR-77-2533)

MÄRSHẢLL, JOHN, JR. (CTR-DO)

$20-14$

HEAT TRANSFER PROBLEMS ASSOCIATED

WITH LASER FUSION

AICHE SYMPOSIUM SERIES

THERMONUCLEAR POWER SYSTEMS.

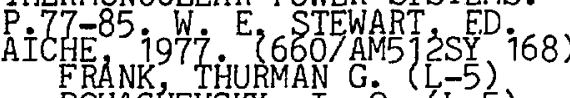

BOHACHEVSKY I. O. $\left(\begin{array}{l}\mathrm{L}-5 \\ \mathrm{~L}-5\end{array}\right)$

PENDERGRASS, JOHN H. (L-5)

20-15

MATERIAL END - PLUGGING FOR THE SCYLLA

IV-P LINEAR THETA PINCH.

ENGINEERING PROBLEMS OF FUSION

RESEARCH. 1977. SYMP. 7TH,

KNOXVILLE, TN. PROC, V.2;

P.1031-4. M.S. LUBELL, ED'

IEEE, 1977. (537/S989EN 1977)

ALSO'PUBLISHED IN:

TIC 79 5P. MN (LA-UR-77-2453)

HANSBOROUGH, LASH D. (MP-14)

COLE THEODORE R. (CTR-4)

COMMISSO ROBERT' J. (CTR-3)

WILLIAMSON, KENNETH D., JR. (CTR-9)
$20-16$

MINIMUM - THICKNESS BLANKET/SHIELD

WITH OPTIMUM TRITIUM EREEDING

AIID SHIELDING EFFECTIVENESS.

TIC, 11P. MN (LA-UR-78-1198) GERSTL, S. A. W. $(T-1)$

$20-17$

NUCLE.ONIC ASPECTS OF SYNFUEL BLANKETS.

LASL,

1978. 8P. (LA-7485-MS)

DUDZIÁ, DONALD J. $(\mathrm{T}-1)$

20-18

NUCLEONIC ASPECTS OF THE LINUS IMPLODING BLANKET.

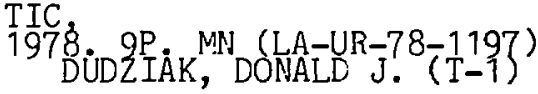

20-19

NUCLEONICS OF THE FAST - LINER

TRANS. AM. NUCL SOC.,

V. 30 P.622-3. 1978 .

SORAN, PATRICK D: (TD-6)
DUDZIAK, DONALD $\mathrm{J}$. (T-1)

$20-20$

PARAMETRIC STUDY OF PLASMA STARTUP

SYSTEMS FOR TOKAMAKS.

ENGINEERING PROBLEMS OF FUSION

RESEA RC.H. 1977. SYMP. 7TH,

KNOXVILLE, TN. PROC.; V.2,

P.1120-31. M.S. LUBELL, ED.

ALSO'PUBLISHED IN:

TIC,
1977 , $4 P$. MN (LA-UR-77-2472)
VOGEL; HERBERT F. (CTR-9)

$20-21$

RIGGATRON NUCLEONICS. ONE -

DIMENSIONAL AHALYSIS.

LASL,

1978: 7P. (LA-7183-MS)

WIENKE, BRUCE R. (T-1)

DUDZIAK, DONALD J. $(\mathrm{T}-1)$

BOSLER,'GERALD E. (T-1)

$20-22$

SCOPING NUCLEONIC STUDIES FOR THE RIGGATRON FUSION REACTOR CONCEPT.

TIC 8 . 8P. MN (LA-UR-78-964)

WIENKE, BRUCE R. (T-1)

DUDZIAK, ${ }_{\text {DONALD J. }}(\mathrm{T}-1)$ 


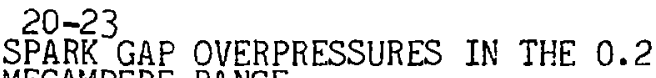

MEGAMPEPE RANGE

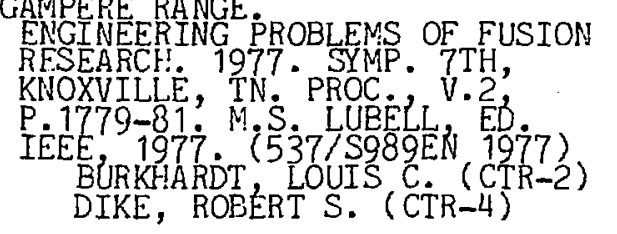

20-24

SURVEY OF LINEAR MAGNETIC

FUSION REACTORS.

1978. 19P. MN! (LA-UR-78-1319)

KRAKOWSKI, ROBERT A. (CTR-12)

$20-25$

THS DOUBLET TOKAMAK OHMIC - HEATING

POWER SUPPLY STUDY.

LASL, 91P. (LA-7053-MS)

ROGERS, JOHN D., JR. (CTR-9) COMP.

$20-26$

TOKAMAK EQUILIBRIA WITH BETA

CLOSE TO 1 .

TIC 1977 . 14P. MN (LA-UR-77-2864)

VAN DER LAAN, PIETER C. (CTR-DO)

MANN, LAWRY W. (CTR-10)

TOROIDAL FUSION REACTOR DESIGN

BASED ON THE REVERSED - FIELD

PINCH.

LASI,

1978: THESIS, IOWA STATE U.

AMES 1978: 96P (LA-7399-T)

$20-28$

TOROIDAL HELICAL QUARTZ - FORMING

MACFINE.

ENGTNEER ING PROBLEMS OF FUSION

RESEARCH. 1977. SYM:P. 7TH,

KNOXVILUE, TN. PROC, V. 1 ,

P. 144-6 M. S. LUBELL ED

ALSO PUBLISHED IN:

TIC

977. 4P. MN (LA-UR-77-2363)

HANKS, KENNETH W.
COLE, THEODORE R.

VOLTAGES IN TOROIDAL PINCH

EXPERIMENTS.

LASL; 13P. (LA-7335-MS)

VAN DER LAAN, PIETER C. (CTR-DO) 


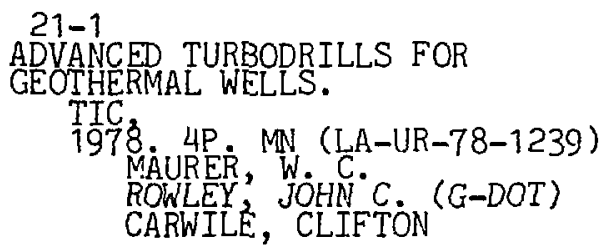


22. GEOTHERMAL ENERGY--ENVIRONMENTAL RESEARCH

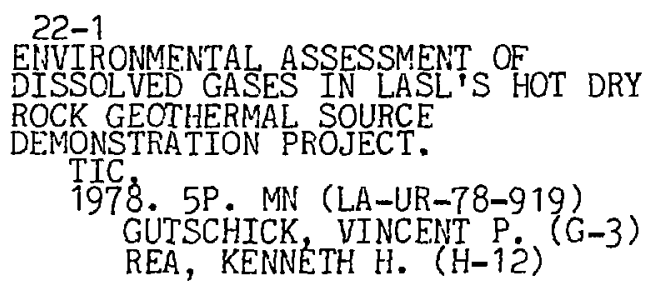


24-1

DEVELOPMENT OF HOT DRY ROCK

RESOURCES.

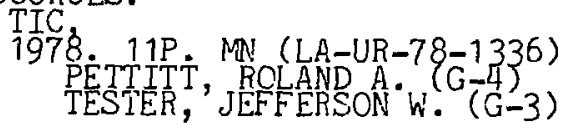

24-2

EXPERIMENTAL ALTERATION OF A

GRANODIORITE IN A CIRCULATION

SYSTEM.

1978. 5P. MN (LA-UR-78-926)

CHARLES, ROBERT W. (CNC-11)

24-3 ${ }^{2}$ OT DRY ROCK - A NEW GEOTHERMAL

ENERGY SOURCE.

ENERGY:639-44. 1978.

MORTENSEN, JEANNETTE J. (G-DOT)

$24-4$

HOT DRY FOCK ENERGY EXTRACTION

OPERATIONS.

TIC, 1978 . 5P. MN (LA-UR-78-904)
HENDRON, ROBERT H. (G-4)

HOT-5 DRY ROCK GEOTHERMAL ENERGY

DEVELOPMENT PROJECT. ANNUAL REPORT,

FISCAL YEAR 1977.

LASL, 294P (LA-7109-PR)

HOT DRY ROCY, AN ALTERNATE

GEOTHERMAL ENJERGY RESOURCE

A CHALLENGE FOR INSTRUMENTATION.

TIC7\&, 14P. MN (LA-UR-78-707)

DENNIS: BERT R: (G-4)
HORTON, EVERETT H. (G-4)

24-7

ALAN:OS HOT DRY ROCK ENERGY SYSTEM.

TIC
1978 SMITH, MORTON C. (G-DOT)

$24-8$

LASL HOT DRY ROCK GEOTHERMAL

ENERGY DEVELOPNENT PROJECT.

1978. 4P. MN (LA-UR-78-906)

HILL, JAMES H. $(\mathrm{G}-4)$
24-9

PROCEEDINGS OF HOT DRY ROCK

GEOTHERMAL WORKSHOP, APRIL 2)-21,

1978 HELD AT LOS ALAMOS SCIENTIFIC

LABORATORY, LOS ALAMOS, NEW MEXICO.

1978.' 63P. (LA-7470-C)

ELSNER, DORIS B. (G-3) COMP.

SEISMIC REFLECTION SURVEYS NEAR LASL GEOTHERMAL SITE. LASL

1978. 17P. (LA-7228-MS) KINTZINGER PAUL R: (G-6) REYNOLDS CHARLES B. SUHR, G.

24-11

THERMAL DRAWDOWN AND RECOVERY OF SINGLY AND MULTIPLY FRACTURED HOT DRY ROCK RESERVOIRS.

LASL,

1978: 15P. (LA-7219-MS)

WUNDER, RAINER

MURPHY, HUGH D. (G-4)

THERMAL EVOLUTION MODELS FOR THE VALLES CALDERA WITH REFERENCE TO A HOT DRY ROCK GEOTHERMAL EXPERIMENT

J. VOLCANOL. GEOTHERM. RES.,

V. 3, P. 197-218. 1978 . KOLSTAD, CHARLES D. $(Q-12)$

24-13

THERMAL STRESS CRACKING AND

ENHANCEMENT OF HEAT EXTRACTION

FROM ERACTURED GEOTHERMAL RESERVOIRS.

TIC, 4P. MN (LA-UR-78-917)

MURPHY, HUGH D. (G-3)

$24-14$

THERMAL STRESS CRACKING AND THE

ENHANCEMENT OF HEAT EXTRACTION FROM

FRACTURED GEOTHERMAL RESERVOIRS.

LASL,

1978: 17P. (LA-7235-MS) 
24-15

URANIUM HYDROGEOCHEMICAL AND STREAM

SEDIMENT RECONNAISSANCE OF THE

CHEYENNE NTMS QUADRANGLE,

WYOMING.

1978. 67P. AND PLATES.

(LA-7237-MS)

TREXIER, PAMELA K. (G-5) 
25-1

CHAP-II. A TRANSIENT SIMULATION

TENE THE FORT ST. VRAIN HIGH

CURA TURE GAS COOLED REACTOR.
NUME SYMPOSIUM. 1978 LOS ALAMOS,

P.90-1. LASL, 1978. (LA-7417-C)

SECKER PHILLIP A.
LAZARUS, ROGER $\mathrm{B} .(\mathrm{Q}-6)$

FUEL-2 PARTICLE COATING DATA.

LASL

1977' 37P. (LA-6558-MS)

HOLLABAUGH, CHARLES M. (CMB-3)

WAGNER, PAUL (CMB-8)

WAHMAN', LYLE A. (CMB-8)

WHITE, RALFH W. (CMB-8\}

CASEO

ENERGY CONVERSION ENGINEERING

SAN DIEGO, CA. PROC, V.2,

P. 1306-11: SAE 1978:

(621.31/E56C $1978 \mathrm{~V} \cdot 2)$

HELMICK, HERBERT H. (Q-8)

25-4

IMPROVED GRAPHITE MATRIX FOR

COATED-PARTICLE FUEL.

LASL

1978. 14P. (LA-7423)

SCHELL: DONALD H. $(\mathrm{CMB}-6)$
DAVIDSON, KEITH V. (CMB-6)

$25-5$

LASAN. A GENERAL PURPOSE

TRANSIENT SYSTEMS ANALYSIS PROGRAM.

CUBE SYMPOSIUM. 1978. LOS ALAMOS,

NM ABST J.J. RUMINER COMP

SECKER, PHILLIP A. JR $(Q-6)$

$25-6$

ROLE OF TRANSITION AND LANTHANIDE

METAL CARBONYLS IN VARIOUS HTGR

SAFETY - RELATED PROBLEMS.

THERMAL REACTOR SAFETY.

1977 TOPICAL MEETING, SUN VALLEY,

ID PROC ${ }_{1977} \cdot 3-332-3-356$. ANS,

BEHRENS, ROBERT G. (CMB-3)
SUVI] 7 S. A CIRCULATING AND PLATEOUT ACTIVITY PROGRAM FOR GAS - COOLED REACTORS WITH ARBITRARY RADIOACTIVE CHAINS.

LASL, 1978 . AND MICROF ICHE.

(LA-OTZ32-MS)
APPERSON, COURTNEY E., JR. (Q-1 


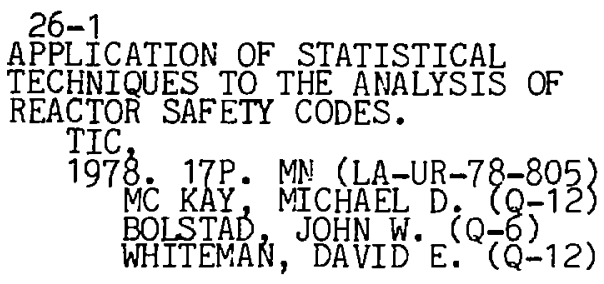

$26-2$

MAMPLE CHANGERS

MAINTENANCE MANULAL.

1978

MYERS, THOMAS A. (CNC-11)

BACKĞ 26 ROUND CROSS SECTION METHOD

AS A GENERAL TOOL FOR REACTOR

ANALYSIS.

TIC

MAC FARLANE ROBERT E- (T-2)

KIDMAN RUSSELL B. (T-2)

LABAUVE, RAPHAEL J. (T-2)

BECKER, MARTIN

26-4

COCONVERSION OF URANIUM-PLUTONIUM

NITRATE SOLUTION TO OXIDE, JULY 1 -

SEPTEMBER 30, 1978.

$$
\begin{aligned}
& \text { 1978. 19P. (LA-7530-PR, VOL. 1) } \\
& \text { BOWERSOX DAVID F. (CMB-11) } \\
& \text { COBB, DONALD D }(\dot{Q}-4) \\
& \text { DAYEM HASSAN ALY }(Q-4) \\
& \text { ENSSLIN, NORBERT }(Q-1) \\
& \text { SHIPLEY, JAMES P. (Q-4) }
\end{aligned}
$$

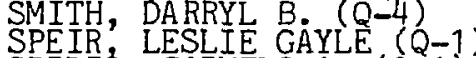

$$
\begin{aligned}
& \text { SPIRIÓ, CARMELO A. (Q-1) } \\
& \text { TAPE JAMES W. }{ }^{(Q-1)}
\end{aligned}
$$

\section{6-5}

COMPUTATIONAL PROCEDURES FOR

MULTIDIMENSIONAL CORE ANALYSIS.

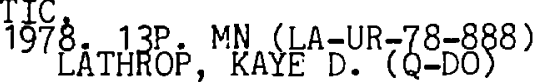

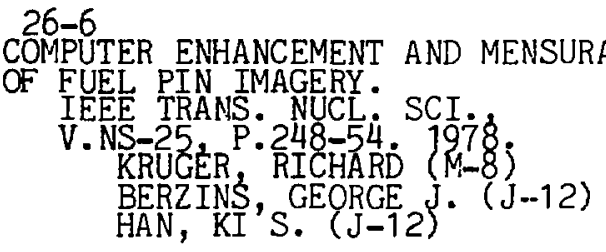

COSTS OF ELECTRONUCLEAR FUEL PRODUCTION.

LASL,

1978: 8P. (LA-7382-MS)

FLATM; THERESA $(S-2)$

$26-8$

FINE ENERGY CROSS SECTION SPACE SHIELDING FOR DOUBLYY HETEROGENEOUS REACTOR CORES.

TRANS. AM NUCL SOC.,

V. 30. P.716-7. i978. STAMAAELATOS, MICHAEL G.

26-9

GASEOUS FUEL REACTORS FOR POWER SYSTEMS.

197

$$
\text { 78. TP. MN (LA-UR-78-1437) }
$$

SCHWENK', FRANCIS C.

26-10

HEAT PIPE NUCLEAR REACTCRS

FOR SPACE APPLICATIOHS.

197 8P. MN (LA-UR-78-643)
KOENIG, DANIEL R $\{0-13)$
RANKEN, WILLIAM A. (Q-13)

26-11

INDUSTRIAL TOMOGRAPHY APPLIED TO REACTOR SAFETY. TIC
KRUGER; RICHARD (M-8)

26-12

INER CONCRETE HEAT TRANSFER

STUDY FOR NUCLEAR POWER PLANT CONTAINMENTS.

$$
\begin{aligned}
& \text { LASL, 46P. (LA-7089-MS) } \\
& \text { GIDO, PICFARD G. (Q)6) }
\end{aligned}
$$

26-13

NEW CONDENSATION MASS REMOVAL MODEL FOR CONTAINMENT AN/LYSIS.

TRANS. AM. NUCL. SOC.,

V. 30 P. 372-4. 1978 GIDO, RICHARD G: $(Q-6)$ 


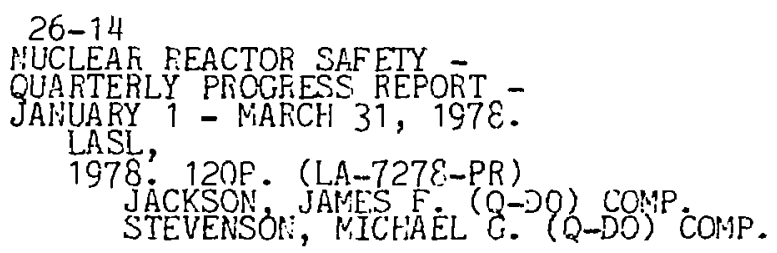

26-15

NUCLEAS REACTOR SAFETY. QUARTERLY

PROGRESS REPORT, APRIL 1 -

JUNE 30, 1978.

LASL, 183P. (LA-7481-PR)

JACKSON, JARE
STEVENSON, MICHAEL G. (Q-DO) COMP.

$26-16$

PINHOI $\because$ IMAGING OF A TEST

FUEL ELEMENT AT THE TRANSIENT

REACIOR TEST FACIIITY.

NUCL. SCI. El:G.

V. 65,2 . $28-40.3978$.

EERZINS, GEORGE j. $(J-12)$

HAY, KI'S. $(J-12)$

$26-17$

REACTOR LOSIMETRY CALIPRATIOMS IN

THE BIG TEN CRITICAL ASSEMELY.

REACTCR DOSIMETRY. 1977

(OCT.) ASTM-EURATOM SYMP. 2ND,

PALO ALTO. PROC. DOSIMETR

WETHODS FOR FUELS CLADDIIIG,

AND STRUCTURAL MATER TALS.

P. 1205-6. (NUREC/CP $-0004 \mathrm{~V} \cdot 3$ )

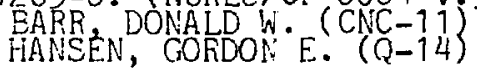

$26-18$

REACTOR TECHNOLOGY - JANUAFX -

VIARCH 1978.

LASL,

1978: 43P. (LA-7316-PR)

WARREN, JOHN L.. (Q-DO) ED.

REACTCR TECHNOLOGY, APRIL-JUNE 1978.

LASL, 31P. (LA-71449-PR)

WAFFEN JOHN L. (Q-DO) ED.

$26-20$

FEACTOR TECHNOLOGY, JULY - SEPTEMEER

1977

LASL,

1978 41P. (LA-7137-PR)

WARREN, JOHN L. (Q-DO)

26-21

REACTOR TECHINOLOGY, OCTOBER-DECEMBER 1977

LASL,

1978. 35P. (LA-7210-PR)

WARREN; JOIN L. (Q-DO) ED.
TRANISPORT AND REACTOR THEORY, OCTOBER 1-DECEMBEF 31, 1977.

LASL

23P. (LA-7131-PR)

MILLER, WARREN F., JR. (T-1)

DUDZIAK, DONALD J' $(T-1)$

GOMEZ, MARY FRANCES $(T-1)$

TRANSPORT AND REACTOR THEORY, JANUARY 1-MARCH 31, 1978.

LAST; 3OP. (LA-7271-PR)

MILLER, WARREN F., JR. (T-1) COMP DUDZIAK, DONALD J' $(T-1\}$ COMP. Q"DELL, R. DOUGLAS $(T-1)$ COMP.

$26-24$

TRANSPORT AND FEACTOR THEORY, APRIL 1 - JUNE 30, 1978.

LASL

978. 24P. (LA-7435-PR)

MILLER, WARREN! F., JR. (T-1)

DUDZIAK, DONALD j: $(\mathrm{T}-1)$

O"LELL $R$. DOUGLAS $(T-1)$

ALCOUFEE RAYMOND E. $(\mathrm{T}-1)$

TRANSPORT AND REACTOR THEORY, JULY 1 - SEPTEMEER 30, 1978. LASL

978, 3CP. (LA-7554-PR)

MILLER, WARREN F., JR. (T-1)

DUDZIAK, DONALD 'j'. $(T-1)$

O"DELL, R. DOUGLAS $(T-1)$

ALCOUFFE, RAYMOND E. (T-1)

GOMEZ, MARY FRANCES $(\mathrm{T}-1)$

26-26

TRISTAN I - TECHNIQUES, CAPABILITIES AND ACCOMPLISHMENTS.

TIC
$1977.24 P . M N(L A-U R-78-523)$
TALBERT, W. L., JR. (Q-14) 
27. GENERAL, MISCELLANEOUS, AND PROGRESS REPCRTS (NONNUCLEAR)

BOOK REVIEW. AMORY B. LOVINS' "SOFT ENERGY PATHS - TONARD A DURABLE PEACE" AND LOVINS' AND PIERCE'S "NON-NUCLEAR FUTURES THE CASE FOR AN ETHICAL ENERGY TRATEGY" ENVIRON WANAGE.

V.2 P. $365-6.1978$ COX, KENNETHE. (CMB-3)

\section{7-2}

COMPUTER ASSISTED COST ESTIMATIMG AT

LOS ALAMOS SCIENT IF IC LABORATORY.

AESOP CONFERENCE PROC

V.17. EOSTON, SEPT. 1977.

(CONF-770937)

ALSO PUBLISHED IN:

197 TOP, MN (LA-UR-77-2070)
SPOONER JAMES E.
STUTZ, ROGER ALAN

$27-3$

E-DIVISION SEMIANNUAL REPORT,

JANUARY 1 - JUNE 30, 1978.

LASL

1978: 76P. (LA-7502-DR)

KEATON, P.W'

$27-4$

E-DIVISION SEMIANNUAL REPORT, JUIE 1 DECEMBER 31, 1977 .

IASL, 89P. (LA-7149-PR)

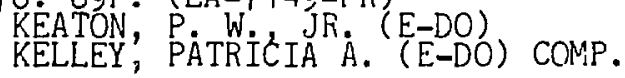

$27-5$

ENERGY DEVELOPMENT VS WATER

QUALITY IN THE UPPER COLORADO

AND UPPER MISSOURI RIVER BASINS.

LASL

1978: 76P. (LA-7497-MS)

27-6

ENERGY SYSTEMS AND STATISTICS,

OCTOEER-DECEMBER 1977 .

1978: 27P. (LA-7240-PR)

SNYDER, ELIZABETH A. (Q-12) COMP.

ENERGY SYSTEMS AND STATISTICS,

JANUARY - MARCH 1978.

LASL, 24P. (LA-7.62-PR)

SIYDER, ELIZABETHA. (Q-12) COMP.
ENE-8 SEPTEMEER 1977 . LASI, 2LP. (LA-7154-PR) SIYYDER, ELIZABETH A. $(0-12)$ COW

GUIDEBOOK FOR NEWS MEDIA RELATIONS. LASL 1978: 19P. (LASL-77-28)

PUBLIC INFORMATICN OFF ICE

$27-10$

HIGH-TEMPERATURE ELECTRONICS

WORKSHOP. PROCRESS IN THE DEVELOPMENT

OF WICROELECTROIIICS FOR THE 500 DEGRE

CENT IGRADE ENUIRONMENT HFLD AT

LOS ALAYOS SCIENTIFIC LABORATCRY,

LOS ALAMOS, NEW NEXICO, JANUARY

4-6, 1978 .

$1973: 5 \mathrm{P}$ (LA-7409-C)

MC CORMICK, J. FYRON (E-4) ED.

27-11

IMPLEMENTATION OF DISTRIBUTED

PROCESSING FOR ADMJPISTRATIVE

APPLICATIONS.

TIC 8 . 16P. MN (LA-UK-78-1100)
HALL, JAKES W. (AADP-DO)

$27-12$
LAMPF PROPOSAL STATUS AND
SUMIVARIES.

1978: 239P. (LA-07444-SF)

RAYECRN, LOIS L. (WP-DO) CCNP.
TALLEY, EEVERLY H. (NF-DO) COMP

$27-13$

LASL FY 1978 SLPPORTING RESEARCH PROGRAM.

1978: 13P. (LA-7471-FS)

HANVEL, ELWAKD F., JR. (DIK OFF) MERLAN, STEPHEl J'
FREIWALD, DAVID A. (DIR OFF

$27-14$

WECHANISMS FOR LASL/UNIVERSITY INTERACTIONS.

LASL

78 15P (LASL-77-31)
FTEINALL TAVIE A- (DIR-C)
LAYMEIN, EDWARE R. (PER-DO) 
$27-15$

MICROFILM INDEX AND RETRIEVAL

TECHNIQUES.

ARMA Q. 12 iD. 4, P. 23-6, 40. 1978.

$27-16$

MODULARIZED SIMULATION OF ELECTRIC

CAR PERFORMANCE

CUBE SYMPOSIUM. 1978. LOS ALAMOS,

NM. ABST. J.J. RUMINER COMP

NACHAMKIN JACK (E-DOR

BOBBETT, RONALD E (E-4)

DEROUIN, CHARLES (E-DO)

LYNN, DAVID $(E-4)$

MC CORMICK, J. BYRON (E-4)

PUBLICATIONS OF LASL RESEARCH, 1977.

1978: 189P. AND MICROFICHE.

(LA-7248-MS)

PETERSEN, LOIS F

27-18

SENSITIVITY ANALYSIS TECHNIQUES.

A CASE STUDY.

LASL,
1978. 29P. (LA-7549-MS)
MC KAY MACHAEL D $(\mathrm{S}-1)$
FORD, ANDREW (S-2)
MOORE, GLENN H. (Q-12)
WITTE, KATHLEEN H. (C-3)

$27-19$

SYSTEMS, ANALYSIS, AND ASSESSMENT,

APRIL - JUNE 1978.

1978. 28P. (LA-7526-PR)

RONQUILLO, ELIZABETH A. (S-DO) COMP.

SYSTEMS, ANALYSIS, AND ASSESSMENT,

JULY - SEPTEMBER, 1978.

1978. 26P. (LA-7573-PR)

RONQUILLO, ELIZABETH A. (S-DO) COMP.

WELCOME TO LOS ALAMOS...

LASL, 54P. (LASL-77-25)

MC FARLAND, LARRY C. (ISD-6) COMP.

FREIWALD, DAVID A. (DIR-O) COMP. 
28-1

ASSESSING NONNUCLEAR INTERCEPTOR

LETHALITY

CUBE SYMPOSIUM. 1978. LOS ALAMOS,

N. 48. LASL, J. 1978 . (LA-7417-C)

MERSON, JEAN L. (WX-8)

TUCKER, EDWIN $\dot{k}$. (WX-8 1 ( WX

28-2

LONG RANGE PROJECTIONS, 1977-1983.

I. THE DIRECTOR'S STATEMENT CONGRESS. 95TH. 2ND SESSION.

HOUSE OF REPRESENTATIVES. COMMITTEE

ON SCIENCE AND TECHNOLOGY. ROLE OF

THE NATIONAL ENERGY LABORATORIES IN

ERDA AND DEPARTMENT OF ENERGY

OPERATIONS - RETROSPECT AND

PROSPECT. REPORT. P. 156-63. GPO,

1978. (328/C95. 2 78-4)

$$
\text { AGNEW, HAROLD M. (DIR) }
$$

28-3

MID-INFRARED OPTICALLY PUMPED

LASERS.

LASL,

1978: 16P. (LA-7331-MS)

$$
\text { JONES, CLAUDE R. (AP-2) }
$$

28-4

MK 12A/W78 AFT SUPPORT EVALUATION.

CUBE SYMPOSIUM. 1978. LOS ALAMOS,

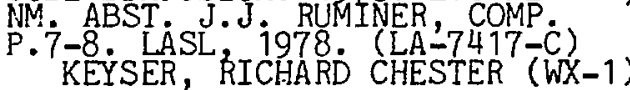

$28-5$

NUCLEAR FISSION ENERGY FUEL

CYCLE OPTIONS.

LASL

MINI-REVIEW. 1978. 4P.

LASL-78-15)

FREIWALD, DAVID A. (DIR-O)

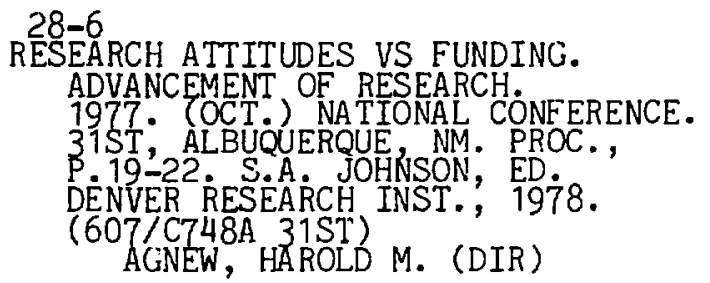

U.S-7 DEFENSE PROBLEM AS IT PERTAINS BAS IELD NUCLEAR WEAPONS.

1978, 13P. (LA-7173-MS) 
29. GEOTHERMAL ENERGY

$29-1$

EXPERIMENTAL GECTHERMAL LOOP, 1 M295 DEGREE C STUDY.

1978 . $44 \mathrm{P}$. (LA-7334-MS)

CHARLES, ROBERT W. (CNC-11)

GEOTHERMAL ENERGY - FURNACE AT OUR FEET.

LASL

1977: UNPAGED. (LASL-77-23)

LOS ALAMOS SCIENTIF IC LABORATORY 
$30-1$

ADVANCES IN ALPHA AIR MONITORING INSTRUMENTATION.

T1978. 13P. MN (LA-UR-78-1150) VALLNTINE, ALLEN M. $(\mathrm{H}-1)$

\section{$30-2$}

DVANCES IN HUMAN INTERNAL

RADIATION COUNTING AT LOS ALAMOS. VULTIPLE SIMULTANEOUS IN - VIVO

MEASUREMENTS.

${ }_{1978}$

UMBARGER, C. JOHN $(\mathrm{H}-1)$

WEST, LEON, JR. $(\mathrm{H}-1)$

30-3 30 RESEARCH AND DEVELOPMENT ZELATED TO HEALTH HAZARD ANALYSIS JULY 1, 1976-JUNE 30, 1977.

1978

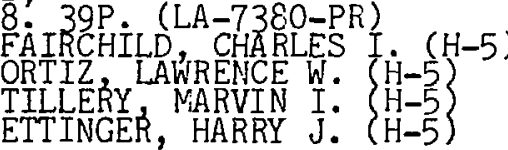

\section{$30-4$}

EROSOL SAMPLING AND CHARACTERIZATION OR HAAZARD EVALUATION OCTOBER 1,
$976-$ SEPTEMBER 30, 1977. LASL,

SCRIPSICK RONALD C. $(\mathrm{H}-5)$

TILLERY MARVIN I. (H-5) STAFFORD RONALD $\dot{G}(\dot{H}-1)$ ROMERO, PABLO 0 . $(\dot{H}-1)$

\section{$30-5$}

IR SAMPLING AND ANALYSIS OF ARCINOGENS. LASL

MINI-REVIEW. 1978. 4P; (LASL-78-6) MORALES, RAUL $(\mathrm{H}-5)$

$30-6$

ALIBRATION OF PERSONNEL DOSIMETERS.

1977. 17P. MN (LA-UR-77-2613)

STORM, ELLERY ( $\mathrm{H}-1)$

CORTEZ JOSEPH R. $(\mathrm{H}-1)$

LITTLEJOHN, GEORGE J. $(\mathrm{H}-1)$
COMPARISON OF A SCDIUM CHLORIDE AEROSOL FILTER TEST METHOD TO SILICA - DUST AND SILICA - MIST FILTER TEST METHODS. AM. IND. HYG ASSOC. J., V. 39 P P $709-16.1978$ (H-5) REVOIR, WILLIAM H.

$30-8$

CONTROLLED - AIR INCINERATION

STUDIES AT THE LOS ALAMOS

SCIENTIF IC LABORATORY. 197

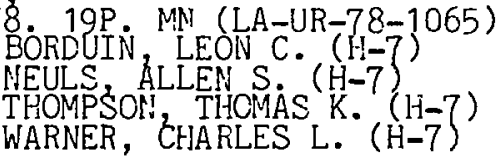

$30-9$

DEVELOPMENT OF AIR - MONITORING

TECHNIOUES USING SOLID SORBENTS

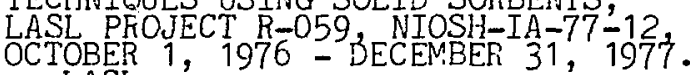

LASL

$1978,41 P$. AND APPEND.

WOOL GE GRY O (H-5) PROJECT DIRECI

30-10

DEVELOPMENT OF SAMPLING AND ANALYTICAL METHODS FOR CARCINOGELSS

OCTOBER 1, 1976-DECEMBER 31, 1977.

LAS: $24 P$. (LA-7375-PR)

MORALES, RAUL (

STAMPFER, JOSEPH F., JR. $(\mathrm{H}-5)$

HEFMES, ROEERT E. $\left\{\begin{array}{l}1 \\ -5 \\ -5\end{array}\right)$

ETTINGER, HARRY $\mathrm{J}$. $(\mathrm{H}-5)$

30-11

ENVIROINENTAL AND RADIOLOGICAL

SAFETY STUDIES OCTOBER 1 - DECEMEER

31.1977 INTERACTION OF PLUTONIUM

238 DIOXIDE HEAT SOURCES WITH

TERRESTRIAL AND AQUATIC ENVIRONMENTS.

LASL,

1978; 31P. (LA-7172-PR) WATERBURY, GLFNN R. (CMB-1) COMP.

$30-12$

ENVIRONMENTAL AND RADIOLOGICAL

SAFETY STUDIES JANUARY 1 - MARCH 31 ,

1978 INTERACTION OF PLUTONIUM -238

AND AQUATIC ENVIRONMENTS.

LASL

$1978 ; 33$, (LA-7309-PR) WATERBURY, GLENA R.' (CME-1) COMP. 
30-13

ENVIRONMENTAL AND RADIOLOGICAL

SAFETY STIDIES JULY 1-SEPTEMBER 30,

SOURCES WITH TERRESTRIAL AND

AQUATIC ENVIRONMENTS.

LASL,

1978: 37P (LA-7577-PR) (CMB-1) COMP.

$30-14$

EIVIRONMENTAL AND RADIOLOGICAL SAFETY

STUDIES, APRTL 1 - JUNE $30,1978$.

INTERACTION OF PLUTONIUM DIOXIDE -238

HEAT SOURCES WITH TERRESTRIAL AND

AQUATIC ENVIRONMENTS.

LASL, 36 P (LA-7420-PR) (CMB-1) COMP.
WATERBURY, GLENN R. (CMB-1)

$30-15$

ENVIRONMENTAL SURVEILLANCE. AT LOS

ALAMOS DURING 1977.

LASL, 83P (LA-7263-MS)

ENVIRONMENTAL SURVEILLANCE GROUP

$30-16$

INDUSTRIAL HYGIENE CONTROL OF TOXIC

MATERIALS IN RESEARCH LABORATORIES.

AMERICAN CHEMICAL SOCIETY.

NATIONAL MEETING 1978 (MAR.

175TH, ANAHEIM 2 CA. ABSTRACTS OF

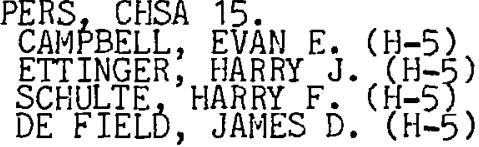

IPTECRATED MINICOMPUTER ALPHA

ANALYSIS SYSTEM.

$$
\begin{aligned}
& 1978.33 P . M N(L A-U R-77-2968) \\
& \text { ASILIK, DENNIS G: }(\mathrm{H}-1) \\
& \text { COY DALE E. } \\
& \text { HENDERSON, RICHARD } \mathrm{W} \cdot(\mathrm{H}-1) \\
& \text { ROMERO, LEONARD L. (H-1) } \\
& \text { THOMSON, DEBRA ANN }(\mathrm{H}-1)
\end{aligned}
$$

30-18

MULTIPLE HEPA FILTER TEST METHODS,

JANUARY - DECEMBER 1977.

19.8. 31P. (LA-7489-PR)

SCHUSTER BURTON G. $(\mathrm{H}-5)$

KYJE THOMAS GAIL (II-5)

OSETEK, DANIEL J.

30-19
MULTIPLE-MIRROR AXIAL CONF INEMENT
USIIG THE FLUID MODEL.

LASL, 11P. (LA-7580-MS)

MILLER; GUTHRIE (CTR-3)
30-20

NEUTRON RESPONSE OF A NEW ALBEDO NEUTRON DOSIMETER.

TIC 3 . 18P. MN (LA-UR-78-40) BLACKSTOCK ALBERT W. (H-1) COPTEZ, JOSEPH R. (H-1)

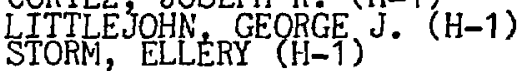

$30-21$

PERMEATION OF METHANOLIC

AROMATIC AMINE SOLUTIONS

THROUGH COMMERC IALLY AVA ILABLE

GLOVE MATERIALS.

$$
\begin{aligned}
& \text { AM. IND. HYG. ASSOC. J., } \\
& \text { V. 38 P2 T21-5. } 1977 \cdot(\mathrm{H}-5) \\
& \text { WEEKS, ROBERT W. }(\mathrm{H}-5) \\
& \text { DEAN, BRENDA J. }
\end{aligned}
$$

30-22 AND BETA RESPONSE OF A NEW THERMOLUMINESCENT DOSIMETER BADGE. TIC

1977 17P. MN (LA-UR-77-3001) STORN, ELLERY $(H-1)$ LITTLEJOHN, GEORGE J. (H-1)

30-23,

PLAGUE! THE SHOCKING STORY OF A DREAD DISEASE IN AMERICA TODAY. CHARLES SCRIBNER'S SONS GREGG, CHARLES T.

$30-24$

PRELIMINARY STUDY OF RADIUM CONTAMINATED SOILS.

LASL,

45P. (LA-7391-MS)

HEALY, JOHN W. (H-DO)

RODGERS, JOHN C. (H-12)

30-25 PART I. DEVELOPMENT OF AN ANTHROPOMETRIC TEST PANEL. AM. IND. HYG. ASSOC. J.,

V. $39 \stackrel{P}{P} .970-5.91978$ MC CONVILLE, JOHN T.

$30-26$

RESPIRATOR STUDIES FOR THE DOE DIVISION OF OPERATIONAL AND ENVIRONMENTAL SAFETY OCTOBER 1, 1976-SEPTEMBER 30, 1977. LASI, 1978 ' 71 P (LA-6969-PR) BRADL $Y$ ORUT D' TRUJILLO, ANDRES $\mathrm{G}$. $(\mathrm{H}-5)$ DOUGLAS, DARREL D. (H-5) 


\section{0-27}

RESPIRATOR STUDIES FOR THE NATIONAL

INSTITUTE FOR OCCUPATIONAL SAFETY

AND HEALTH, JANUARY 1-DECEMBER 31 ,

1977 ASSL

$1978.42 \mathrm{P}$. HEW PUBLICATION NO.

(NIOSH) $78-161$ (LA-7317-PR)
LOWRY PHILIP L. $(\mathrm{H}-5)$

RICHARDS, CHARLES P. (H-5)

GEOFFRION LOUIS A * $(\mathrm{H}-5)$

YASUDA, STANLEY $K \circ(H-5)$

WHEAT, LLOYD D.

BUSTOS, JOSE M: $\mathrm{H}-5$

DOUGLAS, DARREL D. (H-5) PROJECT MANAGER

RESPIRATOR STUDIES FOR THE NUCLEAR

REGULATORY COMMISSION, OCTOBER 1 , 1976 -

SEPTEMBER 30, 1977. PROTECTION FACTORS

FOR SUPPLIED' - AIR RESPIRATORS.

LASL,

HACK, ALAN L. (H-5)

BRADLEY, ORVIL D. $(\mathrm{H}-5)$

TRUJILLO, ANDRES G. (H-5)

$30-29$

SENSITIVE, YET SIMPLE, PLUTONIUM WOUND

MONITOR

HEALTH PHYS.

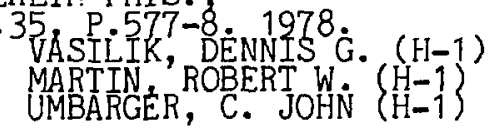

$30-30$

STUDIES ON PERSONS EXPOSED TO PLUTONIUM.

1978

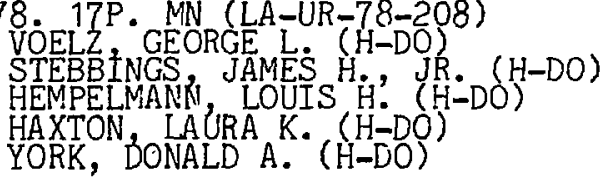

$30-31$

TANDEM HEPA FILTER TESTS.

AM. IND. HYG. ASSOC. J. ,

SCHUSTER BURTON $\dot{\mathrm{G}}(\mathrm{H}-5)$ 
31. HEATING AND COOLING--RESIDENTIAL AND COMMERCIAL APPLICATIONS

$31-1$

BUILDING ENVTROMMENTAL SYSTEMS

EVALUATION FOR THE NATIONAL SECURITY

AND RESOURCES STUDY CENTER.

LASL, 149P THESIS SEVER

INST: OF TECH. WASHINGTON UNTV., ST.

LOUIS, MO. 1978 (LA $-7141-T)$
TERRY, NICHAEL T. (ENG-9)

COMPONENT - BASED SIMULATOR FOR

SOLAR SYSTEMS.

$$
\begin{aligned}
& 1978.5 P \text {. MN (LA-UR-78-1494) } \\
& \text { ROSCHKE MARK A ( wX }(w-4) \\
& \text { DIAMONOD, STEPHEN C. }(W X-4)
\end{aligned}
$$

DATA $31-3$ ACOUTSTION AND MONITORING SYSTEM FOR LOS ALAMOS NATICNAL SECURITY AND RESOURCES STUDY CENTER.

1978. 6P. MN (LA-UR-78-677)

$31-4$

DATA ACGUISTIOR AND PROCESSING

WITH A HP $9 \overline{8} \overline{5}$ "DESK TOP

CALCULATOR CONTROLLED

INSTRUIENTATION SYSTEM IN THE

LOS ALAMOS SOLAF MOBILE /

MODULAR HOME.

TIC
1978.99 P MN (LA-UR-78-773)
HEDSTROM, JAMES C. $(Q-11)$

$31-5$

E.CONOMICS OF THE ATTACHED SOLAR

GREENHOUSE FOR HOME HEATING.

T1C $12 \mathrm{P}$. $\mathrm{MH}$ (LA-UR-78-22)

ALSO PUEL ISHED IN:

TECHNOLOGY FOR ENERGY

CONSERVATION. 1978. NATIONAL

CONF, ALBUQUERQUE NM PROC., (333.9/T255E 1978$)$

$$
\text { KOLSTAD, CHARLES D. (Q-12) }
$$

\section{$31-6$}

EIERGY SAVINGS FOR A SOLAR

HEATED AND COOLED BUILDING

THROUGH ADAPTIVE OPT IMAL

CONTROL.

1978. 23P. NN (LA-UR-77-1048)

FARRIS; DONALD R. (E-4)

MELSA, JAMES L.
$31-7$

ERDA FACILITIES SOLAR DESIGN HANDBOOK.

$$
\begin{aligned}
& \text { TIC } \\
& \text { 1977. 181P. NN }(L A-U R-77-186) \\
& \text { HUNN, BRUCE D. }(W X-4)^{-18}
\end{aligned}
$$

$31-8$

HYBRID PASSIVE/ACTTVE SOLAR HOUSE. FIRST YEAR PEREOFMANCE OF THE HUNN RESIDEHCE.

$$
\begin{aligned}
& 1978.6 P \cdot M N(L A-U R-78-965) \\
& \text { HUNN, } B R U C E D .(W X-4)
\end{aligned}
$$

IMPACTS OF THE NATIONAL ENERGY PROGRAM ON SOLAR ECONOMICS.

$$
\begin{aligned}
& \text { TIC } \\
& 1978.70 P \text { MN (LA-UR-78-131) } \\
& \text { BEIN-DAVID SHAUL } \\
& \text { NOLL, SCOTT } \\
& \text { ROACH, JAMES E. }(Q-12) \\
& \text { SCHULZE, WILLIAM }
\end{aligned}
$$

$31-10$ INITIAL OPERATION AND PERFORMANCE ABSORPTION CHILLER IN THE NATIONAL SECURITY AND RESOURCES STUDY CENTER.

$$
\text { TIC, }
$$

$$
\begin{aligned}
& \text { 78. 6P. MN (LA-UR-78-930) } \\
& \text { HEDSTROM, JAMES C }(Q-11) \\
& \text { MURRAY, HUGH S }(\dot{E}-4) \\
& \text { BALCOME, J. DOUGLAS }(Q-D O)
\end{aligned}
$$

$31-11$

ON-LINE PERFORMANCE CALCULATIONS

FOR THE LOS ALAMOS NATIONAL

SECURITY AND RESOURCES STUDY CENTER.

1978. 8P. MN (LA-UR-78-754) MURRAY HUGH S. $(E-4)$
HEDSTROM, JAMES C. $(Q-11)$

31-12 A GENERAL SIMULATION PROGRAM FOR PASSIVE SOLAR ENERGY.

1978: 50P, (LA-7433-MS) MC FARLAND, ROBERT D. (Q- 1) 
31-13

PASSIVE TESTING AT LOS ALAMOS.

TIC 878 . $6 \mathrm{P}$ MN (LA-UR-78-1158)

BALCOMB J. DOUGLAS (Q-DO)

MC FARLAND, ROBERT D. $(Q-11)$

MOORE, STANLEY W. $(Q-11)$

$31-14$

SIMPLE EMPIRICAL METHOD FOR

ESTIMATING THE PERFORMANCE OF A

THERMAL STORAGE WALL TYPE.

TIC \& 8P. MN (LA-UR-78-1159)

BALCOMB J. DOUGLAS $(Q-D O)$

$31-15$

SOLAR COLLECTOR RELATED RESEARCH AND

DEVELOPMENT IN THE UNITED STATES

FOR HEATING AND COOLING OF BUILDINGS.

1978. 39P. MR' (LA-UR-78-1263)

COLLIER, ROBERT K. $(\mathrm{Q}-11)$

$31-16$

SOLAR ENERGY RESEARCH AT LASL

JULY 1, 1976-SEPTEMBER 30, 1977.

LASL,

1978. 109P. (LA-7115-PR)

BANKSTON CHARLES A., JR. $(Q-11)$ COMP.

NEEPER, DONALD A. $(\dot{Q}-11)$ COMP.

31- 17

STATE OF THE ART IN PASSIVE

TIC 7 . 9P. MN (LA-UR-78-774)

BALCOME, J. DOUGLAS (Q-DO)

$31-18$

VERIFICATION METHODOLOGY FOR THE

DOE-1 BUILDING ENERGY ANALYSIS

COMPUTER PROGRAM.

197

$5 P$ MN (LA-UR-78-1493)
DIAMOND STEPHEN C. (WX-4)
HUNN BRUCE D. (WX-4)

MC DÓNALD, THOMAS E. (E-4) 
$32-1$

ABSORPTION OF INFRARED RADIATION

BY A LAFGE POLYATOMIC MOLECULE.

CARBON DIOXIDE LASER IRRADIATION OF

DISULFUR DECAFLUORIDE.

$$
\begin{aligned}
& \text { J. CHEM. PHYS. } \\
& \text { V.69, P. } 1858-54.1978 . \\
& \text { LYMAN, JOHN L. }(A P-3) \\
& \text { LEARY, KEVIN M. }(A P-3)
\end{aligned}
$$

AMMONIA LASER OPTICALLY PUMPED WITH AN

IIF LASER.

OPT COMMUN

$$
\begin{aligned}
& \text { V.24 P } 27-3 C \text {. } 1978 . \\
& \text { JONES CIALR R. (AP-2) } \\
& \text { BUCHWALD MELVIN I. (AP-2) } \\
& \text { GUNDERSEN, MARTIN } \\
& \text { BUSHNELL, ANDREN H. }
\end{aligned}
$$

$32-3$

ANALYSIS OF LASER FUSION TARGETS

USING MONOCHROMATIC X-RAY

MICRORADIOGRAPHS.

LASL,
1978 25P, (LA-7534-MS)
WHITMAN ROLLIN L. $(M-8)$
DAY ROBERT H. (J-14)
KRUGER, RICHARD (M-8)
STUPIN, DAVID M. $(L-7)$

ANTAR

OPTICAL DESIGN AND ANALYSIS.

INERTIAL CONFINEMENT FUSION.

1978 TIEPICAL MEETING,

TECHNICAL' PAPERS P. TUC9-1.

OSA 1977 (537/I42C)

REICHELT, WALTER H. $(L-10)$

MUNROE, JAMES L. $(\mathrm{L}-10)$

JONES, KENNETH $C$. $(L-10)$

SAXMAN, A. C. $(L-10)$

$32-5$

ANTARES PROTOTYPE POWER AMPLIFIER FINAL REPORT.

I 1978 S 109P. (LA-7186)

LELAND, WALLACE T. $(\mathrm{L}-10)$

YORK, G. $(\mathrm{L}-10)$

GANLEY, JAMES T. $(L-10)$

SWANSON, DAVID A. $(L-10)$

YODA $G$ GAVOKIK, EDWARD J. $(L-10)$

RIEPE KENNETH B. $(\mathrm{L}-10)$

LINDSTRAND, RICHARD $(\mathrm{L}-10)$
COMLY, JACK C. $(\mathrm{T}-6)$
$32-6$

APPLICATION OF CHEMICAL VAPOR DEPOSIT TO THE FABRICATION OF LASER IUSION TARGETS.

INERTIAL CONFINEMENT FUSION.

1978. TOPICAL MEETING,

SAN DIECO, CA. DIGEST' OF

TECHNICAL PAPERS, P. THE7-1.

OSA 1977 . (537/142C)

MC CREARY WILLIAM J. (CMB-6)

CATLETT, DUANE S. ( $\mathrm{CMB}-6)$

$32-7$

APPLICATIONS OF INERTIAL

CONFINEMENT FUSION.

INERTIAL CONFINEMENT FUSION.

1978. TOPICAL MEETING,

SAN DIEGO, CA. DIGEST' OF

TECHNICAL' PAPERS P. WA4 - 1 .

OSA 1977 . (537/142C) BOOTH, LAWRENCE A. (L-5)

$32-8$

ASSESSMENT OF DYNAMICAL PARASITICS

IN THE EBS.

1978 19P, (LA-7166-MS)
ELLIOTT, C. JAMES (L-6)

BASIICS OF MAGNETIC FUSION.

LASL

MINI-REVIEW. 1978. 4P.

(LASL-78-3) 1978.

FREIWALD, DAVID A. (DIR-O)

BATCH PROCESSING TECHNIQUES FOR

MICROBALLOON FUSION TARGETS

INERTIAL CONFINEMENT FUSION.

1978 TOPICAL MEETING,

TECHNICAL' PAPERS, P. TUE 14-1.

OSA, 1977 (537/142C)

BONGIANNI W L L
BUTLER, SUSANNAH (L-7)
MILLER, JOHN R. (L-7)

BROA-11

POWER CARBON DIOXIDE LASERS.

$$
\begin{aligned}
& \text { OPT. LETT. } \\
& \text { V.2 } 2 \text {. } 39.41 .1978 . \\
& \text { CZUCHLEWSKI STEPHEN J. (L-9) } \\
& \text { NOWAK, ANDREW V (L-9) } \\
& \text { FOLEY EDWARD (L-9) }(\mathrm{L}-9) \\
& \text { FIGUEIRA, JOSEPH E. }
\end{aligned}
$$




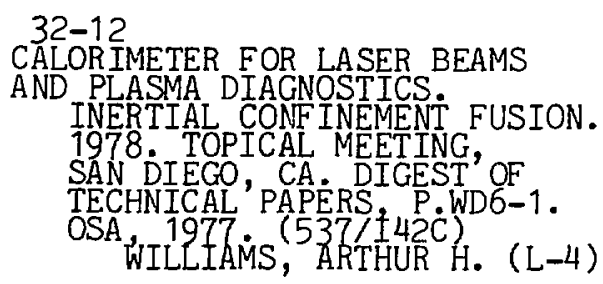

\section{CA2-13 DIOXIDE LASER \\ SYSTEMS STUDIES.}

INERTIAL CONF INEMENT FUSION.

1978 TOPICAL MEETING,

TECHNICAL' PAPERS P. WC13-1 .

OSA 1977 . ( $5371 \pm 22 \dot{C})$

JANSEN, HANSJORG $(L-5)$

CA2-14

ALIGNMENT

INERTIAL CONFINEMENT FUSION.

1978. TOPICAL MEETING,

SAN DIEGO, CA. DIGEST' OF

TECHNICAL'PAPERS P. TUC5-1.

MANNING JOHN P.

KLINGLER, QUENTIN G.

STUMP, CHA RLES J. ( JR,

CÅRBON DIOXIDE-LASER FUSION.

TIC
1978 . 12P. MN (LA-UR-78-1351)
STARK, EUGENE E. (L-5)

\section{$32-16$}

CGARACTERIZATION OF P-TYPE

GERMANIUM FOR 10 MICROMETER

REGION PASSIVE ISOLATION AND

PULSE SHAPING.

INERTIAL CONE INEMENT FUSION.

1978. TOPICAL MEETING,

SAN DIECO, CA. DIGEST' OF

TECHNICAL PAPERS P. TUC1-1.

$O S A$ 1977. (537/142C) PHIPPS; CLAUDE R. JR. (L-9) THOMAS, SCOTT J. (L-9)

COMPRREHENSIVE STUDY OF EXCIMER LASER SYSTEMS.

LASL,

1978. 99P. THESIS, UNIV. OF

NEW MEXICD, ALBUQUERQUE; 1978.

BUTCHER, ROBERT R. (AP-2)
$32-18$

CONTA INMENT VESSEL STRUCTURAL

DESIGN CONSIDERATIONS

INERTIAL CONFINEMENT FUSION.

1978. TOPICAL MEETING,

SAN DIEGO, CA. DIGEST' OF

TECHNICAL' PAPERS, P.WC 12-1 .

OSA, 1977 (537/ $42 \mathrm{C})$

BOHACHEVSKY,$I$. O. $(\mathrm{L},-5)$

32-1

CONTROLLING SURFACE FINISHED IN VACUUN DEPOSITED COATING FOR LASER FUSION TARGETS.

INERTIAL CONFINEMENT FUSION.

19'8. TOPICAL MEETING,

SAN DIEGO, CA DIGEST'OF

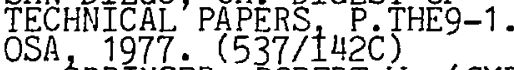
SPRINGER, ROBERT W. (CMB-6)

$32-20$

DAMAGE RESISTANCE OF AR-COATED

GERMANIUV SURFACES FOR NANOSECOND CARBON DIOXIDE LASER PULSES.

TIC, 19P. MN (LA-UR-77-2530)

NEWNAM BRIAN E. (AP-2)

$32-21$

DAMAGE RESISTANCE OF COATED OPTICS FOR PULSED CARBON DIOXIDE LASERS. TIC.
1978. 18P. MN (LA-UR-78-931)
NEWNAM, BRIAN E. (AP-2)

32-22

DERIVATION OF THE PHYSICAL

EQUATIONS SOLVED IN THE INERTIAL

CONE INEMENT STABILITY CODE DOC.

LASL

1978: 20 P. (LA-7214-MS) CRANFILL, CHARLES W. (L-6)

32-23 3 ENGINEERING OF LARGE HIGH PRESSURE GAS LASER AMPLIFIERS.

TIC 8 . 17P. MN (LA-UR-78-876)

ALSO PUBLISHED IN :

ADVANCES IN LASER TECHNOLOGY

(EMPHASIZING GASEOUS LASERS) 1978

SEMINAR, WASHINGTON, D.C. PRCC.,

P. 39-45, D. FINKLEMAN ED.

SPIE 1978 (621.366/A244L)
LELAND, WALLACE T. (L-10) 
$32-24$

DESIGN OE THE ENERGY STORAGE SYSTEM FOR THE HIGH ENERGY GAS LASER

FACILITY AT LASL

ENGINEERIMG PROBLEMS OF FUSION

RESEARCH. 1977. SYMP. 7TH,

KNOXVILLE, TN. PROC. V.2'

IEEE, 1977. (537/S989EN 1977)

ALSO PUBLISHED IN:

197

$$
\begin{aligned}
& 72 \text { 12P. MN (LA-UR-77-2419) } \\
& \text { RIEPE, KENNETH B. }(L-10) \\
& \text { KIRCHER, MAPY (L-10) }
\end{aligned}
$$

\section{$32-25$}

DESIGN OF THE POWER AMPLIF IER

FOR THE HEGLF AT LASL

ENGINEERING PROBLEMS OF FUSION

RESEARCH. 1977. SYMP. 7TH, KNOXVILLE, TN. PRCC. V. 1 ,

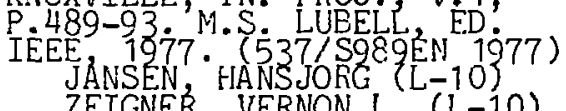

ZEIGNER, VERNON L. $(\mathrm{L}-10)$

$32-26$

DEVELOPMENT OF BROADEAND GAS

ISOLATOR MIXES FOR HIGH POWER

CARBON DIOXIDE LASERS.

INERTIAL CONFINEMENT FUSION.

1978 . TOPICAL MEETING,

TECHNICAL PAPERS P. TUC2-1.

$O S A, 1977 .(537 / 142 C$

NOWAK, ANDREW $V .(L-9)$

CZUCHLEWSKI STEPHEN J. (L-9)

FIGUETRA, JOSEPH F. (L-9)

$32-27$

DEVELOPNENT OF CRYOGENIC

TARGETS FOR LASER FUSION.

TIC,
ALST PUP PUISHED (LA-UK-77-1523)

ADV. CRYOG. ENG.

V. 23 PR. $676-81.1978 .(0-10)$

DEVICE FOR MICROPARTICLE ARRAY

PREPARATION.

REV. SCI: INSTRUM.

V. 49 P P. 1488-9. 1978. J. (L-7)

\section{$32-29$}

DIELECTRIC TARGET FABRICATION FOR ELECTRON BEAM FUSION.

INERTIAL CONFINENENT FUSION.

1978. TOPICAL MEETING,

SAN DIECO, CA. DIGE.ST'OF

TECHNICAL'PAPERS, P. TUE4-1.

OSA, 1977 ( $534 / 142 \mathrm{C})$

NEWFIELD, STEPF EN E. (CMB-6)
DIFFKACTION MEASUREMENTS FOR LASL'S ANTARES LASER SYSTEM.

LASL: 32P. (LA-7443-MS)

SOLLID; JON E. $(L-10)$

$32-31$

DISCHARGE PUMPED EXCIMER LASERS.

LASER AND ELECTROOPTICAL SYSTEMS.

1978 OSA/IEEE CONFERENCE,

TECHNICAL'PAPERS, NO.WBB1, (6233-40\% OSA 1978 \% FAHLEN, T. S.

$32-32$

DISTR IBUTED MICROCOMPUTER CONTROL SYSTEM FOR A HIGH - ENERGY GAS LASER FACILITY

MICRO 77 COMPUTER CONF. RECORD, APRIL, 1977 , P. 99-104. STRATT, BOBBY G. $(\mathrm{L}-10)$ HONG, JUNG PYO (L-10)

DOUBLE - SHELL TARGET DESIGNS FOR THE LOST ALAMOS SCIENTIFIC LABORATORY EIGHT -BEAM LASER SYSTEM.

LASL, 13P. (LA-7167-MS) KINDEL JOSEPH M. (L-6)
STROSCIO, NICHAEL A. (L-6)

$32-34$

EFFECT OF CARBON DIOXIDE LASER

PULSE MODE QUALITY ON MUL TIPLE PHOTON ABSORPTION IN SULFUR HEXAFLUORIDE.

OPT. COMMUN.

V. $25, P$ P $391-4.1978$.

LYMAN JOHN LA (AP-3)

FISHER, ROBERT A. $\left\{\begin{array}{l}\mathrm{L}-9 \\ \mathrm{~L}-9\end{array}\right.$

EFFICIENT GAS ISOLATOR FOR CARBON DIOXIDE 10 MICPOMETER P BRANCH LASER OPERATION.

ELECTRO-OPTICSS/LASER 77

CONFERENCE AND EXPOSITION.

1977 ANAHEIM, CA. PROC',

SCIENTIFIC COIFERENCE MANAGEMENT, 1977. (535/E38OL 1977) CZUCHIEWSKI, STEPHEN J. (L-9)

NOWAK, ANDREW $V$. $(\mathrm{L}-9)$
FOLEY, EDWARD $(\mathrm{L}-9)$ 
$32-36$

ELECTRON BEAM INITIATED FUSION - TARGET FABRICATION.

INERTIAL CONFINEMENT FUSION.

1978. TOPICAL MEETING,

SAN DIECO, CA. DIGEST' OF

TECHNICAL' PAPERS P.TUE3-1.

OSA 1977 SIMONSIC (537'́ $42 \mathrm{C}$ ) (CMB-6)

MIX, L. PAUL JR:

ENERGY EXTRACTION AND PULSE

SHAPING IN ANTARES.

INERTIAL CONF INEMENT FUSION.

1978. TOPICAL MEETING,

SAN DIEGO, CA. DIGEST' OF

TECHNICAL'PAPERS P. TUC10-1.

OSA, 1977 . $(537 / 142 \mathrm{C}$

WOLFE PETER N. $(L-10)$

FELDMAN, BARRY J. (L-9)

KNAPP' CHARLES E. $(\mathrm{L}-10)$
VOLKIN, HOWARD C. $(\mathrm{L}-5)$

$32-38$

ENERGY-TRANSPORT EXPERIMENTS IN 10 PLASMAS.

J. APP. PHYS.

V. 49 P. $3851-4$. MITCHELL KENNETH B. (L-1)
GODWIN, ROBERT P. (L-4)

32-39

ENVIRONMENTAL EFFECTS AND POTENTIAL

HAZARDS OF LASER FUSION ELECTRIC

GENERATING STATIONS.

INERTIAL CONFINEMENT FUSION.

1978. TOPICAL MEETING,

SAN DIEGO, CA. DIGEST' OF

TECHNICAL PAPERS P. WC8-1.

OSA 1977. (537/142C)

DEVANEY JOSEPH J. $(\mathrm{L}-5)$

BOOTH, LAWKENCE A $(\mathrm{L}-5)$

FRANK, THURMAN $G$. $(\dot{L}-5)$

32-40

FABRICATION OF COMPLEX CRYOGENIC

TARGETS

INERTIAL CONFINEMENT FUSION.

1978. TOPICAL MEETING,

SAN DIECO, CA. DIGEST' OF

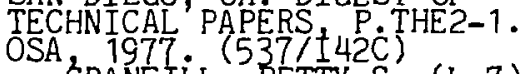

CRANFILL BETTY S. ( $L-7)$

FARNUM EUGENE H. $(\mathrm{L}-7)$

LOWE, ARTHUR T. $\left\{\begin{array}{l}\mathrm{L}-7 \\ \mathrm{LILLER}, \mathrm{JOHN}\end{array}\right.$
$32-41$

FARADAY EFFECT IN MERCURY $(1-X)$

CADMIUM $(X)$ TELLURIDE AT CARBON

DIOXIDE LASER WAVELEHGTHS.

J. APPL. PIYYS.

V. 49 , P :2265-7': 1978 AHRENKIEL, RICFARD $K$. ( $(L-Q)$ WEISS, PAUL B. (L-9)
WATKINS, DAVID $w$. (TD-2) GULATI, S. ${ }_{\dot{w}}$.

$32-42$

FIBER OPTICS AND MICRCPROCESSORS - A

CONTROL - SYSTEM SOLUTIO! EOR THE.

LASER - FUSION ENVIROMMEITT.

TIC
1978 THUOT, MICHAEL E. $(\mathrm{E}-4)$

FI'-42-43 OPTICS ANIC MICROPROCESSORS. A CONTROL - SYSTEM SOLUTION FOR THE LASER - FUSICN ENVIRONMENT.

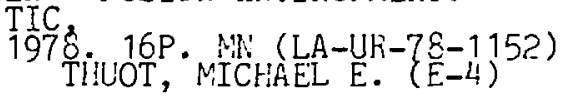

$32-44$

GENERAL ICF REACTOR DESIGN

CONSIDERATIONS.

INERTIAL COIFINEMENT FUSICN.

1978. TCPICAL MEETING,

SAN' DIEGO, CA. DIGE.ST' $C F$

TECHNICAL PAPERS P $\because C 2-1$.

$O S A, 1977 .(537 / 142 \dot{C})$

BOHACHEVSKY I BOTH, LAWEEICE $A$ : $\{L-5\}$

FRANK, THLRMA! C." (L-5)

HAFER', JCFH F. $(\dot{L}-5)$

PENLERGRASS, JOHE: H. (L-5)

HIUH - EFFICIE:ICY PULSED

10.6 MICROMETER PHASE - CClJUGATE

REFLEC'IIOH: VIA TEGEMERATTE

FCUR - WAVE VIXING.

VPT. LETT. F.82-4. 1978

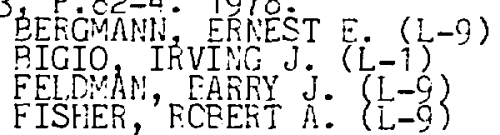

$32-46$

HIGH - ENEFGY, THELVF - CHAME LASES FACILITY (DELFIN) FOH SPILTI TCAI

IRRADIATION: OF THERMOUCLEA TAFCETS. BY N.G. EASCV, ET AL. USSR ACADEYY OF SCIENCES, P.N.LEEEEEV PHYSICS

INSTITUTE. PREPFINT 1.0 .74 . LASL,

1978: 26P. (LA-7114-TR)

BCHACHEVSKY, I. C. (L-5) TFAM:SLAT 
$32-47$

HIGH - RESOLUTION SPECTROSCOPY USING

TUNABLE DIODE LASERS - TECHNIQUES AND APPL IC ATIONS.

T977. 9P. MN (LA-UR-77-2222)

ALSO PUBLISHED IN:

ELECTRO-OPTICS/LASER 77

CONFERENCE AND EXPOSITION.

1977 . ANAHEIM, CA. PROC.,

P.589-96. INDUSTRIAL ANL

SCIENT IFIC CONFERENCE MANAGEMENT,

1977. (535/E380L 1977)

MC DOWELL, RCBIN S. (AP-4)

$32-48$

HYBRID METHOD FOR THE INUMERICAL

SOLUTION CE THE ELECTRON TRANSPORT

EQUATIOI: - THE REDIJCED SOURCE

METHOD

LASL,

1978: 10P: (LA-7410-4S)

LEE KEIMETH

STROSCIO, FICHAEL A. (L-6)

$32-49$

INJECTION OF LASER FUSION: PELLETS.

PART T. ACCURACY REQUIRED,

DEFLECTIONS.

LASL

1978: 12P. (LA-7477-MS)

DEVANEY, JCSEPH J. (L-5)

INIEEISE HIGH - PRESSURE SEQUENCE - EAID

CAREON DIOXIDE LASER.

OPT. LETT. $2.16-8.1978$.

FELDMAN, EARRY J. $(L-9)$
FISHER, ROBERT A. $(\mathrm{L}-9)$
POLLOCK, CLIFFOR̃ R. $(\mathrm{L}-9)$
SIMOIS, SCOTT W. (L-9)
TEFCOVICH, RENATO G. (L-9)

32-51

INTENSITY DEPENDENCE OF INUERSE

BREMISSTRAHLUNG ABSORPTION IN AN

IMHOMOGENEOUS STANDING WAVE.

PHYS. FLUIDS,

V.21 P.793-7. 1978

FAEHL, R. J. $(T-15)$

RODERICK, NORMAN F.

$32-52$

INTERFEROMETRIC MEASUREMENT OF CRYOGENIC

LASER EUSION TARGET UN IFORMITY.

APPL. 17 P $852-3.1978$.

MOLLID; JON E. (L-10)
IUNEOS.

EQUATIOH-OF-STATE ROUTINE.

LASL

1978: 10P (LA-7313-MS) CRANFILL CHARLES $W .(L-6)$ MORE, RICHARD

\section{$32-54$}

LAFGE - AFERTURE DISCHARGES IN E-BEAM SUSTA INED CARBON DIOXIDE AMPL IF IERS.

ENGINEERING PROELEMS OF ELSION

RESEARCH, 1977. SYMP. TTH,

KNOXYILLE TN. PROC, Y.1,

IEEE, 1977 . (537/S989EN 1977)

ALSO' PUELISHED IN:

TIC77. 11P. MN (LA-UR-77-2451)

LELAND; WALLACE T ( $1-10)$

GANLEY, JAMES T. $(L-10)$

XIRCHER, MARY $(\mathrm{L}-10)$

YORK, G. $(\mathrm{L}-10)$

LASER - FUSION PROCRAM AT LOS ALATOOS.

TIC

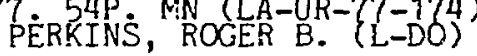

LASER EUSION PROGRAM AT LASL JANUARY 1 - JUNE 30, 1977.

LASL, 155P. (LA-6982-PR)

SKOBERNE, FREDERICK (L-DO) COMP STARK, EUGENE E. (L-5) COMP.

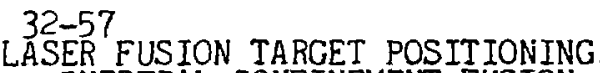

INERTIAL CONEINEMENT FUSION.

1978. TOPICAL MEETING,

SAN DIEGO, CA DIGEST OF

TECHNICAL' PAPERS P. THE10-1.

OSA 1977. ( $537 / 142 \dot{C}$

MILLER JOAN R. (L-7)

DAY ROBERT D. $(\mathrm{L}-1)$

HANSEN, WILFRED G. (L-1)

TUCKER', HOWARD E. $(\mathrm{L}-1)$

$32-58$

FUS INDUCED FUSION

IEEE MINICOURSE. 2ND, TROY, NY.

PROC. G. H. MILEY, ED. 10.1977.

(621.484/F993 1977) GITOMER, STEVEN J. (T-6) 


\section{$32-59$}

LASERS AND POWER SYSTEMS FCR

INERTIAL CONF INEMENT FUSION

REACTORS.

TIC
1978. 24P. WN $(L A-U R-7 \varepsilon-1350)$
STARK, EUGENE E. $(L-5)$

\section{$32-60$}

\section{LASL EIGHT BEAM LASER TARGET}

INSERTION SYSTEN

INERTIAL CONF INEMENT FUSION.

1978. TOPICAL MEETING,

SAN DIEGO, CA. DIGEST OF

TECHNICAL' PAPERS P. TUC6-1.

DAY ROBERT D. (E-4) $(w X-4)$

\section{2-61}

LASL 100-KJ CAREON DIOXIDE LASER

FOR ICF RESEARCH. ANTARES

INERTIAL CONF INE!JENT FUSION.

1978. TOPICAL MEETING,

SAN DIEGO, CA. DIGEST' OF

TECHNICAL PAPERS P. TUC7-1.

OSA 1977 (537/142C)

STRATTON THOMAS F. $(L-10)$

DURHAM, FRANKL IN $\mathrm{P}$ : $(\mathrm{L}-\mathrm{DO})$

JANSEN, HANS JORG $(\mathrm{L}-10)$

LELAND; WALLACE T. $(L-10)$

REICHELT, WALTER H. $(L-10)$

ZEIGNER, VERNON L. $(L-10)$

MASS 32 EJECTION FROM SHOCKED SURFACES

OF LEAD.

INERTIAL CONF INEMENT FUSION.

1978. TOPICAL MEETING,

SAN DIEGO, CA. DIGEST' OF

TECHNICAL' PAPERS P. TUD 15-1.

OSA $1977 .(537 / 42 \dot{C})$

MAH, RICHARD (CMB-6)

\section{$32-63$}

MEASUREMENT OF ALIGNMENT ACCURACY FOR LASER FUSION EXPERIMENTS.

APPL. OPT 17 P.160. 1978.

BENJAMIN ROBERT F. (L-4) WHITE, LARRY $(L-4)$

\section{$32-64$}

MICRORADIOGRAPHY OF LASER FUSION

TARGETS WITH MONOCHROMATIC X RAYS.

INI.RTIAL CONFINEMENT FUSION.

1978 TOPICAL MEETING,

TECHNICAL' PAPERS P. TUE12-1.

$O S A$ 1977. (537/142C)

STUPIN, DAVID M ( $(\mathrm{L}-7)$

DAY ROBERT H. ( J-14)

WRITMAN, ROLLIN L (M- $(M-8)$
$3 \hat{c}-65$

MINIMLM RELEASE, HICH PRESSURE DT FILL SYSTEN:FCR LASER FUSION TARGETS

INERTIAL COLFINEMENT FUSION.

1978. TOPICAL MEETING,

SAI! DIEGO, CA. DIGEST' OF

TECHNICAL PAPERS $P$ P TUE5-1. MARNTMU, EUGENE H. (L-7)

32-65

MIRFOR PCSITION DISPLAY EQUIPMENT

FOR THE TAFGET CHAMBER MIRROR MOUNTS OF THE 8-BEAM LASER FUSION RESEARCH FACILITY.

CUBE SYMPOSIUM. 1978. LOS ALAMOS, NM. ABST. J.J. RUMINER COMP.
P.81-2. LASL 1978 . (LA-7417-C)
WELLS FRANK D. (E-5) WELLS, FRANK D. (E-5)
REMINGTON, DAVID $(E-4)$

$32-67$

MODEKN ULTRAFAST STREAK CAMERA DEVELOPMENT AID APPLICATION REVIEW.

1977. 7P. MN (I.A-UR-77-2474)

ALSO PUBLISHED IN:

ELECTRO-OPTICS/LASER 77

CONFERENCE AND EXPOSITION.

1977. ANAHEIM, CA. PROC.,

P. 336-2. INDUSTRIAL AND

SCIENT IFIC CONFERENCE MANAGEMENT, 1977 (535/E380L 1977 LIEBER, ALBERT (J-14)

$32-68$

NANOSECOND PULSE AMPLIFICATION IN CARBON DIOXIDE AT EFFICIENCIES EXCEEDING 20 PERCENT

INERTIAL CONFINEMENT FUSION.

1978. TOPICAL MEETING,

SAN DIEGO, CA. DIGEST' OF

TECHNICAL PAPERS, P. WC14-1.

OSA, 1977 . (537/142ं

STARK, EUGENE E. ( $L-5)$

LELAND, WALLACE T. $(\mathrm{L}-10)$

VOLKIN, HCWARD C. (L-5)

$32-69$

NEW METHOD FOF PRODUCING

CRYOGENIC LASER FUSICN TARGETS.

ADVANCES IN CFYOGENIC

ENGINEERING, V.23. P.669-75.

K.D. TIMNESFALS, EL. PLEALA, MILLER, JCHN R. (L-7) 
32-70

NEW SATURABLE ABSORBER FCR THE DAREON DIOXI

$$
\begin{aligned}
& \text { APPL. PHYS. LETT. } \\
& \text { V. } 33 \text {. P. } 705-7.91978 . \\
& \text { AHRENKIEL, RICHARD K (L-9) } \\
& \text { FIGUEIRA J JOSEPH F. (L-9) } \\
& \text { DUNLAVY, DONALD J' (E-10) } \\
& \text { THOMAS, SCOTT J. ( } L-9) \\
& \text { SIEVERS, A. J. }
\end{aligned}
$$

32-71

NUMERICAL COMPUTATION OF THE DEMSTTY PROFILE PRODUCED BY 10.6 MICROMETER IRRADIATION OF A SILICON DIOXIDE

MICROBALLOON

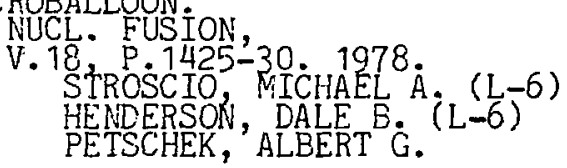

32-72 THE LASL EIGHT BEAM LASER FUSION SYSTEM

INERTIAL CONFINEMENT FUSION.

1978 TOP DICAL MEETING, $O$

TECHNICAL'PAPERS P. TUC4-1.

$$
\text { OSA, } 1977 \text {. } 537 \% \text { T2 } 425)(\mathrm{C}-1)
$$

OPTICALLY PUMPED MIDDLE INFRARED

LASERS

IASTER AND ELECTROOPTICAL SYSTEMS.

1978 OSA/IEEE CONEERENCE,

TECHNICAL PAPERS, NO. THJJ2,

P. 102-4. CSA, 1978

(621.36/L343E 1978)

EUSHNELL ANDREW H.

GUNDERSEN, MARTIN

BUCHWALD, MELVIN I. (AP-2)

$32-74$

CVERVIEW OF LASER FUSION.

NASL -REVIEW. 1978. 4P. (LASL-77-24)

STARK, ELGENE E. (L-5)

PLASMA POLYHERIZED FILNS AS

LASER FUSION TARGETS.

INERTIAL CONFINEMENT FUSION,

1978. TOPICAL MEETING,

SAN DIECO, CA. DIGEST' OF

IECHNICAL'PAPERS P. THE5-1.

COWE ARTHUR T $(L-7)$
PLASMA POLYMERIZED P-XYLENE. AS A

LASER FUSION TARGET.

SLIRF. SCI

V.76; P. $242-56.1978$

LOWE, ARTHUR T. (L-T)

FRIES, RALPH JAY (L-7)

32-77

LASER - FUSION TARGETS.

INERTIAL CONFINEMENT FUSION.

1978. TOPICAL MEETING

SAN DIEGO, CA. DIGEST'OF

TECHNICAL PAPERS F. THE 8-1.

OSA $1977 .(537 / 142 C)$

CATLETT, DUANE S. (CMB-6)

32-78 8 RPARATION OF ULTRATHIN

POLYETHYLENE FOILS BY FILM CASTING.

REV. SCI, INISTRUM.

V. 49 CRANE ILL, BETTY S. (L-7)

32-79

SULF IDE APPLICABLE TO FARADAY

ROTATORS FOR CARBON DIOXIDE

LASER SYSTEMS.

IEEE TRANS: MAGN.

V. $M A G-14, \dot{P} .454-6$ ' 1978. AHRENKIEL, RICHARD K. (L-9)

$32-80$

PKOPOSAL FOR STUDY OF VACUUM ADIABAT COMPRESSION OF A RELATIVISTIC ELECTR BEAM GENERATED BY A FOILLESS DIODE. LASL

1978. 15P. (LA-7169-P)
THODE, LESTER E. (T-15)

32-81 1 TOTY ANTARES POWER AMPLIFIER MODÚLE.

INERTIAL CONFINEMENT FUSION.

1978. TOPICAL MEETING,

SAN DIEGO, CA. DIGEST' OF

TECHNICAL PAPERS P TUC8-1.

GANLEY, JAMES T. (L-10)

LELAND, WALLACE T. $(\mathrm{L}-10)$

SWANSON, DAVID A. (L-10)

YORK, G. (L-1O)

RECENT X-RAY ANALYSIS OF LASER - FUS PLASMA

HIGH-TEMPERATURE PLASMA DIAGNOSTIC 1978 2ND TOPICAT CONF SANTA FE
NM. ABSTRACTS P. 32. (LA-7160-C) NM. ABSTRACTS ${ }_{\mathrm{LEE}} \mathrm{P}, \mathrm{PING}$ 
$32-83$

REQUIREMENTS FOR COMMERCIALIZATION

OF INERTIAL CONEINEMENT FUSION.

INERTIAL CONF INEMENT FUSION:

1978. TOPICAL MEETING,

SAN DIEGO, CA. DIGEST OF

TECHNICAL PAPERS P. WC1-1.

$O S A$ 1977. (537 $142 \mathrm{C})$

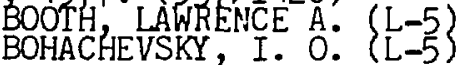

32-84

SECONDARY LASER CAVITY FOR

WEAK ABSORPTIONS.

APPL. OPT $17.29-60.1978$.

KCHUSTER, BURTON G. $(\mathrm{H}-5)$

32-85

PRESSURE CARBON DIOXIDE DISCHARGE. LASER AND ELECTROOPTICAL SYSTEMS.

1978. OSA/IEEE CONEERENCE,

SAN DIEGO, CA. DIGEST OF

TECHNICAL'PAPERS, NO.WEE1,

P.48-9. OSA 1978.

$$
\begin{aligned}
& \text { FELDMAN, BARRY J. }(L-9) \\
& \text { FISHER, ROBERT A. }(\mathrm{L}-9) \\
& \text { POLLOCK, CLIFFORD R. } \\
& \text { SIMONS, SCOTT W. } \\
& \text { TERCOVICH, RENATO G. (L-9) }
\end{aligned}
$$

SIM-86 MODEL FOR EXPLODING PUSHER

TARGETS.

$$
\begin{aligned}
& \text { LASL; } 16 \mathrm{P} \text { (LA-7218-MS) } \\
& 1978 \text { GIOVANIELLI, DAMON V. (L-4) } \\
& \text { CRANE ILL, CHARLES } W .(\mathrm{L}-6)
\end{aligned}
$$

$32-87$

SPACE - CHARGE EFFECTS ON PARTICLE

DIAGNOSTICS OF LASER - PRODUCED

PLASMAS.

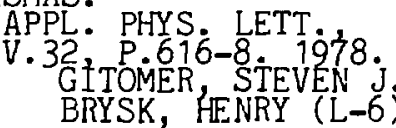

$32-88$

SPACE CHARGE EFFECTS IN LASER

PLASMA PARTICLE DIAGNOSTICS

HIGH-TEMPERATURE PLASMA DIAGNOSTICS.

NM. ABSTRACTS P.40. (LA-7160-C) GITOMER, STEVEN J
32-89

STABILIZATION OF HIGH - GAIN

MULTIPASS POWER AMPLIFIERS USINC

SATURABLE ABSCRBERS. EXPERIENCE ON:

THE. LASL EIGHT - BEAM SYSTEM.

APPL. PHYS. LETT 1 . MONTCOMERY MICHAEL D, (L-i) CARLSON RANDOLPH L. (L-1)

CASPERSON, D. E. (L-1) CZUCHLEWSKI STEPHEM! J. (L-9) FIGUEIRA, JOSEPH E. $\left\{\begin{array}{l}\mathrm{L}-9 \\ \mathrm{LACLUND}\end{array}\right.$ LADISH, JOSEPH'S. (L-1) NINARE, ANCREN V V $(L-9)$

32-90

OF HIGH CAIN

MULTIPASS POWER AMPLIFIERS

USING SATURABLE ABSORBERS.

EXPERIENCE ON THE LASL EIGHT

BEAM SYSTEM.

INERTIAL CONF INEMENT FUSION.

1978. TOPICAL MEETING,

SAN DIEGO, CA. DIGEST OF

TECHNICAL' PAPERS P. TUC 3-1.

OSA 1977 ( $537 /$ I $42 \mathrm{C})$

MONGOMERY MICHAEL D. $(L-1)$

CARLSON RANDOLPH $L .1(L-1)$

CASPERSCH: D. E. (L-1)

FIGUEIRA, JOSEPH E. $(\mathrm{L}-3\}$

HAGLUND R F F. JR.

LADISH, JOSEPH S. $(L-1)$

NOWAK, ANDREN V. $(L-G)$

THOTOA SOHN G. $\left(\mathrm{L}^{-1}\right)(\mathrm{L}-1)$

TIMIERMAIN!, ELGENE $\mathrm{L}$. $(\mathrm{L}-1)$

$32-91$

STREIUTH CPTTMIZATION OF COATINGS USI

MATERIAL LAMINATE TECHNIQUES

INERTIAL COIIFINEMENT FUSION.

1978. TOPICAL MEETING,

SAN DIEGO, CA. DIGEST' OF

TECHIIICAL PAPERS P. THE6-1.

$O S A, 1077 .(537 / 142 \dot{C})$ SPRINGER, RCBERT W. (CH-6)

$32-92$

SYNTHETIC FUEL PRODUCTION

FROIN ICF SOURCES.

INERTIAL CONFINEMENT FUSION.

1978. TOPICAL MEETING,

SAN DIEGO, CA. DIGEST'OF

OSA 1977 . (537) I $42 C$

EOOTH, LAWRENCE A. $(\mathrm{L}-5)$ 
$32-93$

THRESHOLD OF DETECTION FOR VARIOUS

MATERTALS AT 10.6 MICROMETERS.

APPL. OPT

V. $17.2 .2670-1.1978$.

SOLLID, JON E. (L-10)

FOLEY, EDWARD (L-9)

PHIPPS, CLAUDE R., JR. (L-9)

32-94

TRITIUM PROCESSING SYSTEMS FOR

INERTIAL CONF INEMENT FUSION

FACILIT'IES.

INERTIAL CONFINENENT FUSION.

1978. TOPICAL MEETING,

SAN DIEGO, CA. DICEST'OF

TECHUICAL'PAPERS P. WC9-1. PENDERCRASS, JOHN H. (L-5)

TUA:AELE LASERS FOR WASTE MANAGEMENT

PHCTCCHEMISTRY APPLICATIONS.

$$
\begin{aligned}
& 1978,56 P \text { (LA-71 13-MS) } \\
& \text { FINCH, FRANCIS I. (AP-DOT) COMP. }
\end{aligned}
$$

$32-96$

TWO- DIMENSIONAL COMPUTER MODELING AND

AIALYSIS OF THIN - WALL MICROSPHERES.

INERTIAL CONFINEMENT FUSION.

1978 'TOPICAL MEETING,

TECHNICAL PAPERS P. TUE 13-1.

OSA, 1977 . $(537 / 42 \dot{C})$

WHITMAN, ROLLIN L $(M-8)$

KRUCER, RICHARD $(M-8)$
DAY, ROBERT H. $(\mathrm{j}-14)$

STUPIN, DAVID M. (L-7)

ULTRAS

T-KAY CALIBRATION OF

$X$-RAY - DIODES EOR LASER FUSION

PLASMA DIAGNOSTICS.

HIGH-TEMPERATURE PLASMA DIAGNOSTICS.

1978. 2ND TOPICAL CONF SANTA FE,

NM. ABSTRACTS P $\cdot 35 \cdot(\mathrm{j}-14-7160-C)$

DAY, ROBERT $\mathrm{H}_{\mathcal{j}}$ )

ELESEERRY, THOMAS L. $(j-14)$

$32-98$

USE OF PYROELECTRIC VIDICONS

FOR MEASUREMENTS ON CARBOH

DIOXIDE LASER SYSTEMS.

INERTIAL CONF INEMENT FUSION.

1978 TOPICAL MEETING,

TECHNICAL'PAPERS P. TUC 13-1.

OSA, 1977 . (537/142C)

WOHLER, DAVID R, $(\mathrm{L}-4)$
$32-99$

VACUUM UV IMAGING OF A LASER

PRODUCED PLASYMA

INERTIAL CONF INEMENT FUSION.

1978. TOPICAL MEETING,

SAN DIEGO, CA, DIGEST'OF

TECHNICAL'PAPERS P. WD8-1.

EHLER, A. WAYNE $(L-4)$

CARPEVTER, JAMES P. (L-1)

$32-100$

VARIABLE PULSE WIDTH GENERATION

FOR CARBON DIOXIDE LASERS IN

THIE 100-PS TO 1-ISS RANGE.

INERTIAL CONF INEMENT FUSION.

$197{ }^{\circ}$ TOPICAL MEETING,

TECHNICAL'PAPERS, P. TUC11-1. OSA 1977 (537/142C) MC LELLAN, EDWARD J. (L-9)
FIGUEIRA, JOSEPH F. (L-9)

$32-101$

WORKING WITH SAPPHIRE AIND RUBY.

LASER FOCUS

V. 14 NO.8, P.75-8. 1978. LAZAZZERA, VITO J. (A.P-2)

32-102
1/4 JOULE DISCHARGE PUMPED
KRYPTON ELUORIDE LASER.
EEV. SCI. INSTRUM
V. 49 P. T72-4. 1978 .
SLE ROBERT C (AP-2)
SCOTT, PETER B. (AP-2)

32-103

1800 TORR LARGE APERTURE DOUBLE DISCHARGE CARBON DIOXIDE

LASER AMPLIFIER.

INERTIAL CONF INEMENT FUSION.

1978. TOPICAL MEETING,

SAN DIEGO, CA. DIGEST OF

TECHNICAL'PAPERS P. TUC 12-1.

OSA, 1977 ( $537 / 142 \dot{C})$

BROWN J. C

FELDMAN, BARRY J. $\{L-9\}$

SIMONS' SCOTT $W$. TERCOVICH, RENATO G. (L-9) 
33. INSTRUMENTS

$33-1$

AN ATMOSPHERIC OBSERVATORY.

LASL, 4P. (LASL-78-99)

SANDFORD, MAXWELL T
HORAK, HENRY G. $(J-9)$

A 33-2 2 LANCHE TRANSISTOR PULSER FOR FAST GATED OPERATION OF MICROCHANNEL PLATE IMAGE - INTENSIF IERS.

TIC 77 . 8P. MN (LA-UR-77-2402)

ALSO PUBLISHED IN:

IEEE TRANS. NUCL: SCI.

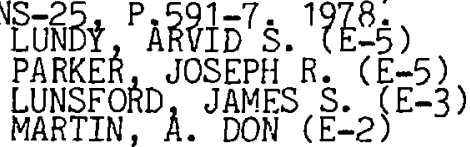

$33-3$

CODED APERTURE IMAGING. PREDICTED

PERFORMANCE OF UNIFORMLY REDUNDANT ARRAYS

APPL. OPTICS,

V. 17 FENIMORE, EDWARE $(\dot{\mathrm{P}}-4)$

$33-4$

INDUSTRIAL WORKSHOP ON LASL

SEMICONDUCTOR RADIATION-DETECTOR

RESEARCH AND DEVELOPMENT.

LASL, 31P. (LA-7408-C)

ENDEBROCK, MARJORIE (E-10) COMP.

INFARED DETECTORS FOR USE AT 10

MIC ROMETERS.

1978

(LA-7276-MS)

JARMIE', NELSON (P-7)

LASL LENS DESIGN PROCEDURE - SIMPLE, FAST PRECISE, VERSATILE.

1978 ,

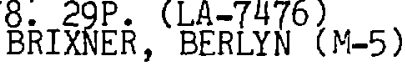

$33-7$

RMS IMAGE SPOT SIZE AND RMS

OPTICAL PATH DIFFERENCE.

APPL. OPT

V. 17 P. $715-6.1978$.

BRIXNER, BERLYN (M-5)
$33-8$

NATIONAL LIVESTOCK ELECTRONIC

IDENTIFICATION SYSTEM.

LASL

MINI-REVIEW. 1978,4 4P; (LASL-78-16)
HOLM, DALE M. (H-6)

NONDESTRUCTIVE IMSULATOR TESTING

SYSTEM

LASL, 11P. (LA-7558-MS)

FULLBRIGHT, HENRY $\mathrm{J}_{0}$, III $\left(\mathrm{N}_{-}-1\right)$

NONS-10

AMALYSIS PROGRAMI FOR CONCRETE

CONTATNMENTS UNDER STATIC,

DYNAMIC, AND LONG - TERM LOADINGS.

LASL,

1978. 131P. (LA-7496-MS) ANEERSON, CHARLES A. (Q-13)

$33-11$

PROCEEDINGS OF THE FUEL CELL IN TRANSPORTATION APPLICATIONS WORKSHOP AUGUST 15-17, 1977 , LOS ALAMOS SCTENTIF IC LABOKA TORY,
LOS ALAMOS, MM 87545 .

LASL, 138P. (LA-7270-C)

MC CORMICK, J. EYROII (E-4) ED. BOBBETT RCNAL MC BREEN, JIMI

$33-12$

PROXIMITY-FOCUS. THE NEW

GEIEERATION STREAK TUEE.

TIC 8 \& 8 , MN (LA-UR $-78-1436)$
LIEBER, ALBERT $(P-3)$

33-13

QUAFTZ CRYSTAL MICROBALANCES. A REFINED TECHNIQUE FOR DETERMINING CHANGES IM MASS LOADING FROM

FREQUENCY SHIFTS.

LASL, 13P. (LA-7106-MS)

FOWLER, MALCOLM N. (CNC-11)

SEDLACEK, WILLIAN A. (CNC-11) 
$33-14$

RESONANTLY ENHANCED FOUR - WAVE

MIXING.

PATENTED

JUNE 12 T 1978, FILED 1977.

BEGLEY, FICHARD F. (AP-4)

SCANNING ELECTRON MICROSCOPE.

LASL

MINI-REVIEW 1978. 4P.

(LASL-78-17)

33-16

TILTED MIRROR UNSTABLE

IEEE J. QUANTUM ELECTRON.

V.QE-14 P. $103-7.1978$

KURNIT, NORNAN A. (AP-2) 
34. ISOTOPE SEPARATION

$34-1$

CARBON DIOXIDE LASER INDUCED

政

J. CHEM. PHYS,

V.68, P:1671-6! 1978

LEARY, KEVIN M. (AP-4)

LYMAN, JOHN L. (AP-4)

ASPREY, LARNED E. (CNC-4)

FREUND, SAMUEL M: (AP-4)

FILLING IN THE GAPS. SHG IN AD-P.

ELECTRO-OPTICS/LASER 77
CONFERENCE AND EXPOSITION.

1977. ANAHEIM CA. PROC.,

P.92-5. INDUSTRIAL AND

SCIENTIEIC CONFERENCE MANACEMENT,

1977. (535/E380L 1977) BALOOG, GEORGE (AP-2)

\section{4-3}

HETEROGENEOUS CONDENSATION OF

BORON TR ICHLORIDE IN THE PRESENCE

OF CARBON DTOXIDE LASER IRRADIATION.

\section{OPT. LETT.}

V.3. P.10-32. 1978

AEE, JAMES T., JR. (AP-1)

$34-4$

HIGH ENERGY LASING OF XENON BROMIDE IM

AN ELECTRIC DISCHARGE.

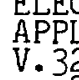

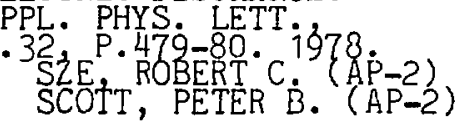

$34-5$

INTRACAVITY INFRARED SECOND

HARMONIC GENERATION USING CADMIUN:

TELLURIDE PLATES.

ELECTRO-OPTICS/LASER 77

CONFERENCE AND EXPOSITION.

1977. ANAHEIM CA. PROC.,

SCIENTIFIC CONFERENCE MANAGEMENT,

1977. $(535$ /E380L 1977$)$

ECKHARDT, ROGER C. (AP-2)

HINSLEY, RONALD (AP-2)
$34-6$

ISOTOPE SEPARATICN IN CARBON

DISULF IDE. AN ARGON FLLUORIDE

LASER APPLICATION.

LASER AND ELECTROOPTICAL SYSTEMS.

1978. OSA IEEE CONFERENCE,

SAN DIEGO, CA. DIGEST OF

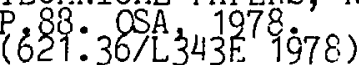

LOREE, THCMAS R. (AP-2)

CLARK, JOHN H. $(A P-3)$

BUTTERF IELD, KEINETH B. (AP-2)

LYMAN, JOHN'L. $(A P-3)$

ENICLEMAN, ROLF, JR. (CME-1)

$34-7$

KILOMETER PATH LOW TEMPERATURE

MULTIPLE REFLECTION CELL FCR

LASER SPECTROSCOPY USING TUNABLE

SEMICONDUCTOR DIODES.

APPL. OPT

V. 17. P. $25311-5.1978 ;$

CRIGGS, JANES E. (AP-1)

PERSCN, WILLIS B. (AP-4)

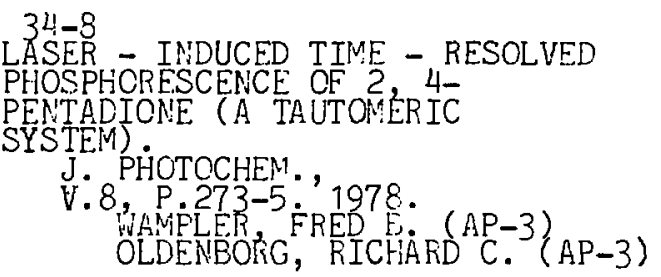

$34-9$

LASEP INDUCED TIME RESOLVED

PHOSPHORESCEHCE OF OXALYL FLUORILE

SSUP $3 / A / S U B$ U/ MOLECULES.

$$
\begin{aligned}
& \text { J.9 PHOTOCHEN.' } 1907-9 \text {. } 1978 . \\
& \text { KAKL ROT-9. 1978. (AP-3) }
\end{aligned}
$$

LASER ISOTOPE SEPARATION

$$
\begin{aligned}
& \text { LASERS IN CHEMISTRY. T977. } \\
& \text { CONF. THE ROYAL INSTITUTION, } \\
& \text { LONDON PROC.; } 184-94 . \\
& \text { N.A. WEST, ED'; ELSEVIER, } 1977 . \\
& \text { (54 } 1 \text {. 35/L343C) } \\
& \text { ROEINSON C. PAUL (AP-DO) } \\
& \text { JENSEN, REED J, (AP-DO) } \\
& \text { CANTRELL, CYRUS D., III (T-12) }
\end{aligned}
$$


$34-11$
LASER SYNTHETIC CHEMISTRY.

TIC, 11P. MN (LA-UR-77-2790)
1977. ${ }^{2}$ (ARY, KEVIN M. (AP-3)
CLARK, JOHN H. (AP-3)

34-12

LIFETIMES OF ELECTRONICALLY EXCITED

URANIUM HEXAFLUORIDE IN THE

PRESENCE OF INORGANIC QUENCHERS.

J. PHOTOCHEM.,

V.9, P 473-9:'1978.

WLDENBORG RICHARD C -3$\}$ AP-3)

RICE, WALTER W. (AP-2)

34-13

MULTIPLE PHOTON ISOTOPE

SEPARATION IN MOLYBDENUM

HEXAFLUOR IDE

CHEM. PHYS. LETT.

FYMUND, SAMUEL MOH $(A P-3)$

OPTICAL Q-BRANCH PUMPING OF THE

P-AND R- BRANCH TRANSITIONS IN

THE NU MODE OF SULFUR

HEXAFLUORTDE.

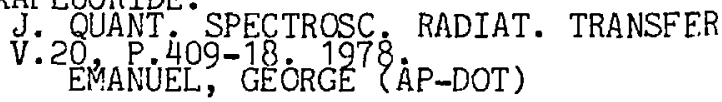

$34-15$

PHOTOC̈HEMICAL REDUCTION OF URANYL

TRI-N-BUTYL PHOSPHATE.

II. TEMPERATURE DEPENDENCE AND

QUANTUM YIELD.

J. INOFG IUUCL. CHEM.,

V. 40, $1895-7 \cdot 1978.2 \quad(A P-4)$

ROFER-DE POORTER, CHERYL K. (AP-4)

$34-16$

PHOTOLYTIC SEPARATION OF D FROM H IN

CRYOGENIC SOLUTIONS OF FORMALDEHYDE.

J. CHEM. PHYS.

V. 69: $1961-\dot{5}$ ' 1978.

MIIER, WILLIAM B., II (AP-4)

FREUND, SAMUEL $\mathrm{N}_{0} \cdot\left(\begin{array}{l}\mathrm{AP}-4 \\ \mathrm{AP}-4\end{array}\right.$

BEATTIE, WILLARD H. (AP-4)

34-17

PURIFICATION OF MATERIALS USING

INFRAPED LASERS. REMOVAL OF

DIBORANE FROM SILANE

ELECTRO-OPTICS/LASER 77

CONFERENCE AND EXPOSITION.

1977. ANA HEIM, CA. PROC.,

P.609. INDUSTRIAL AND

SCIENTIFIC CONFERENCE MANAGEMEN",

$1977.535 / E 380 \mathrm{~L} 1977$

FREUND, SAMUEL M: (AP-3)
34- 18

QUANTITATTVE DETECTION OF TRACE

IMPURITIES IN GASES BY INFRARED

PECTROMETRY OF CRYOGENIC SOLUTIONS

ANAL. CHEM

V. 50, P. $1260^{\circ}-2.1978$.

FREUND, SAMUEL M. (AP-4)

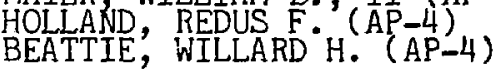

SILANE PURIFICATION VIA LASER APPL. PHYS. LETं

V. 32, P. 46-9. 1978

ANDERSON, ROBERT G. (AP-3)

$34-20$

SPACE CHARGE IN LINEAR

ACCELERATORS WORKSHOP.

LASL, 168P. SPACE CHARGE

If LINEAR ACCELERATORS. 1977.

WORKSHOP LOS ALAMOS NM.

1978. (LA-7265-C)

JAMESON, ROBERT A. (MP-9) ED.
SHREFFLER, L.

$34-21$

SPECTRAL TUNING OF ARGON FLUORIDE AI KRYPTON FLUORIDE DISCHARGE LASERS.

APPL. PHYS LETT 32 P 17 - 19 .

LOREE THOMAS R. (AP-2)

BUTTERFIELD, KENNETH B. (AP-2)

BARKER, DEAN L. (AP-2) 


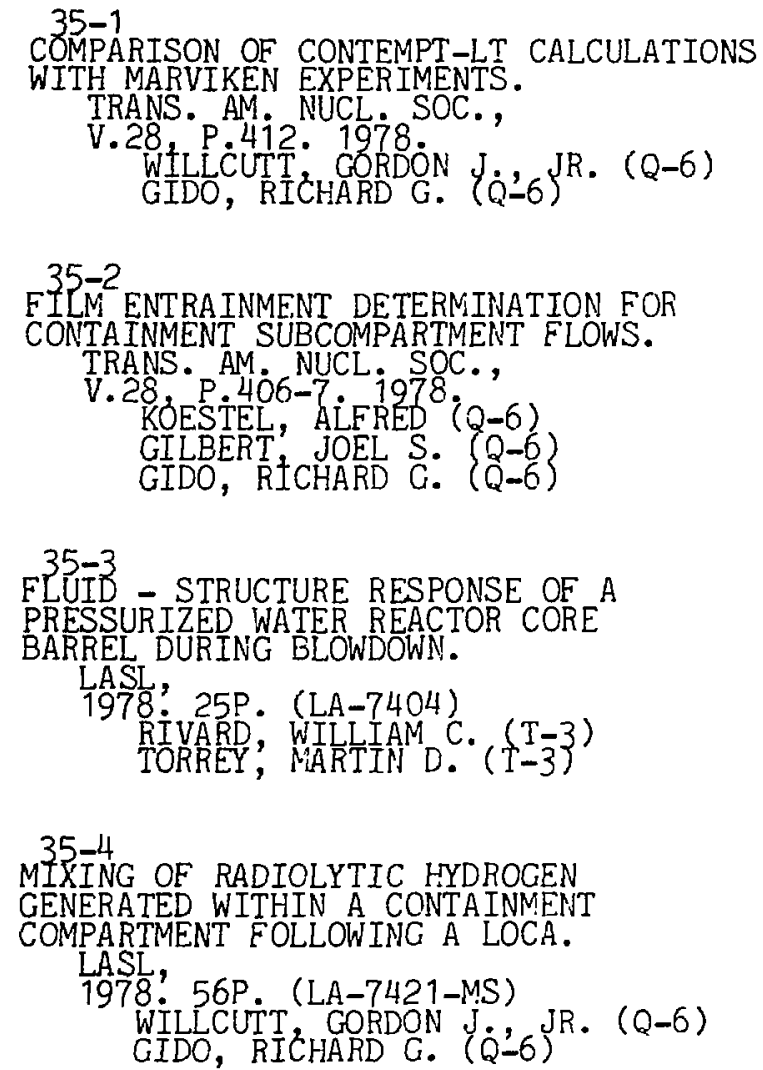

FLUID

PRESSURTZTRUCTURE RESPONSE OF A

BARREL DURING BLOWDOWN.

$$
\begin{aligned}
& \text { LASL, 25P. (LA-7404) } \\
& \text { 1978 }(T-3) \\
& \text { RIVARD; WILLIAM C. }(T-3) \\
& \text { TORREY, MARTIN D. }(T-3)
\end{aligned}
$$

$35-4$

MIXING OF RADIOLYTIC HYDROGEN

GENERATED WITHIN A CONTAINMENT

COMPARTMENT FOLLOWING A LOCA.

$$
\begin{aligned}
& \text { LASL, 56P. (LA-7421-MS) } \\
& 1978 \text {. 56. (Q-6) } \\
& \text { WILLCUTT CORDON J J JR. (Q-6) } \\
& \text { GIDO, RICHARD G. (Q-6) }
\end{aligned}
$$

\section{$35-5$ TCAL STUDY OF APPARATUS}

SCALING IN A PRESSURIZED WATER

REACTOR. A STATUS REPORT - MAY 1978.

LASL; 36P. (LA-7387-SR)

DALY, BART J. $\mathrm{J}(\mathrm{T}-3)$

\section{5-6 \\ PORTABLE ACTIVE NEUTRON INTERROGATION SYSTEM FOR LIGHT - WATER REACTOR FUEL ASSEMBLIES. \\ LASL, \\ 1978 ' 13 P (LA-7528-M) \\ MENLOVE, HOWARD O ${ }^{(Q-1)}(\mathrm{Q}-1)$ \\ (Q) $(Q-1)$ \\ MEDINA, EVITA G. (Q-1)}

$T^{35}-7$ (TRANSIENT REACTOR ANALYSIS

CODE) HYDRODYNAMICS, 7078 . LOS ALAMOS,

M. AOAST. J.J RUMINER, COMP LILES, DENNIS
MAHAFY JOH
WILLIAMS, KEN A:

$(\mathrm{Q}-6)$

$\left(\begin{array}{l}0-6 \\ Q-6\end{array}\right)$

TRAC-81 - AN ADVANCED BEST

ESTIMATE COMPUTER PROGRAM FOR PWR LOCA ANALYSIS 1. METHODS, MODELS, USEF INFORMATION, AND PRCGRAMMING DETAILS. LASL

1978: 385P. (NUREG/CR-0063)

(LA-T2TO-MS, VOL. 1 ) THERVIAL KEACTOR SAFETY GROUP

35-9

TRAC-P 1 POST TEST ANALYSIS OF

U.S. STAMDARD PROBLEM 7 . TRANS. AM N NUCL SCC. PYUN JUNG.JO (Q-6) WILLIAMS, KEN $A .(Q-6)$ 
$36-1$

APPLICATION OF THE SIMMER-1 CODE TO

LOF ACCIDENTS IN AN LMFBR.

LASL, 19P (LA-7194-MS)

HENNINGER, FUDOLPH J. (T-1)

ALCOUFFE, RAYMICND E. $(T-1)$

$36-2$

ENDE/E-IV, LIE-IV, AND THE CSENG

BENCHMARKKS.

LASL: 50P. (LA-7355-MS)

KIDMAN, RUSSELL B. (T-2)

$36-3$

EVALUATION OF HODOSCOPES FOR FUEL MOTION MEASUREMENT IN MULTIPIN

BUNDLES.

TRANS. AM. NUCL SOC. ,

V. $30, P .465-6.1978$.

EVANS, ALEERT E., JR $(Q-14)$

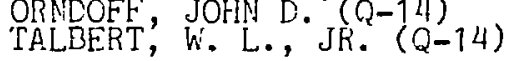

$36-4$

LINEAR, CONTINUOUS TRIANGULAR MESH

DIFFERENCF SCHEME FOR TRANSPCRT

TRAंNS. AM NUCL SOC. ,

V. 28 P. $257-8$ i978

WALTERS, WALLACE F. (T-1)

$36-5$

LOS ALAY:OS FAILLRE MCDEL (LAFM)

A COCE FOR THE PREDTCTION OF LUFER

FUEL PIN FAILURE.

LASL,

978: $43 \mathrm{P}$ : (LA-7161-MS) 
37. LMFBR--FUELS AND MATERIALS ENGINEERING AND DEVELOPMENT:BASE TECHNOLOGY

$37-1$ COMPUTER ANALYSIS AND CONTROL FOR NON DESTRUCTIVE GAMMA SCANNING OF LMFER FUEL PINS.

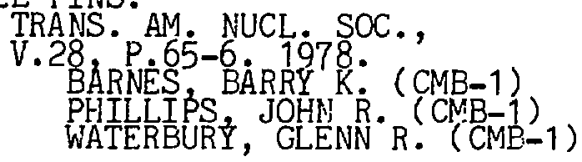

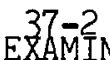

EXAMINATION OF FAST REACTOR FUELS,

FBR ANALYTICAL OUALTTY ASSURANCE

STANDARDS AND METHODS, AND

ANALYTICAL METHODS DEVELOPMENT -

IRRADIATION TESTS, JANUARY 1-

MARCH 31, 1978.

LASL, 9P. (LA-7266-PR)

BAKER; RICHARD D. (CMB-DO) COMP.

\section{$37-3$}

EXAMINATION OF FAST REACTOR FUELS,

FBR ANALYTICAL QUALITY ASSURANCE

STANDARDS AND METHODS, AND

ANALYTICAL METHODS DEVELOPMENT -

IRRADIATION TESTS, APRIL 1-

JUINE $30,1978$.

1978. 11P. (LA-7430-PR)

BAKER, RICHARD D. (CMB-DO) COMP.

$37-4$

EXAMINATION OF FAST REACTOR FUELS,

FBR ANALYTICAL QUALITY ASSURANCE

STANDARDS AND METHODS, AND

ANALYTICAL METHODS DEVELOPMENT -

IRRADIATION TESTS

SEPTEMBER 30, 1978.

1978

BAKER, RICHARD D. ( CMB-DO) COMP.

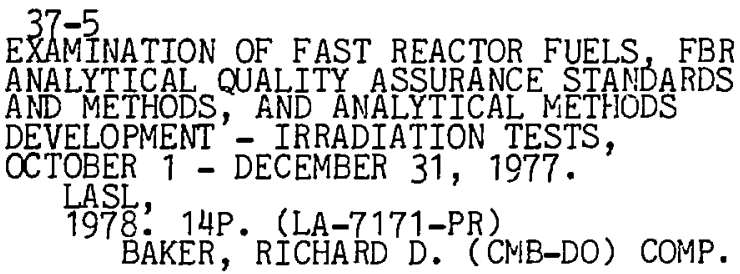

$37-6$

RESULTS OF RESEARCH INTO SUPERCONDUCTI SWITCHES WITH HIGH SPECIFTC

SWITCHING CAPACITY TRANSLATION OF

GLUKHIKH, V.A. ET AL. REZLL'TATY

ISSLEDOVANII SVERKHPROVODIASHCHIKH

KLIUCHEI S VYSOKOI UDEL'NOI

RAZRYVNOI MOSHCHNOST' IU.

LASL, 8P. (LA-TR-78-13)
1977 MACHALEK, MILTON D.' (CTR-2) TRAN 
38. LMFBR--SAFETY TECHNOLOGY

38-1

APPLICATION OF A NONREDUNDANT

PINHOLE ARRAY TO A TEST FUEL ELEMENT

AT TREAT.

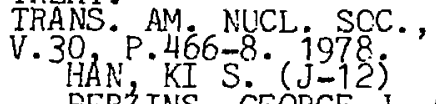

BERŻINS, GEORGE J. (J-12)

$38-2$

COMPUTER SIMULATION OF LMFBR

ACCIDENT SEQUENCES BY SIMMER-II

CUBE SYMPOSIUM. 1978. LOS ALAMCS,

NM. ABST. J.J. RUMINER, COMP

P. 100-1. LASL 1978. (LA-7417-C)

BOHL; WILLIAM R. $(0-7)$

EXPLOZRATORY COMPARISON OF METHODS

FOR COMBINING FAILURE-RATE DATA

FROM DIFFERENT DATA SOURCES.

LASL, 31P. (LA-7556-MS)

MARTZ, HARRY F. JR J $(S-1)$

$38-4$

EXTENDED LIKELIHOOD INFEFENCE IN

RELIABILITY.

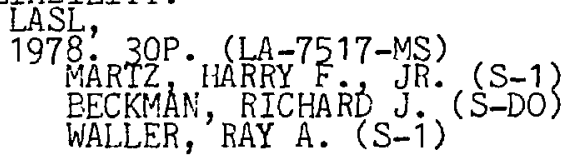

$38-5$

NUCLEAR REACTOR SAFETY QUARTERLY

PROGRESS REPORT, JULY i-SEPTEMBER 30, 1977. LASL,

1978: 77P. (LA-7039-PR)

JACKSON JAMES E (Q-DO) COMP
STEVENSOH, MICHAEL G. (Q-DO) COMP.

$38-6$

ROLE OF MECHANISTIC ANALYSIS IN

PROVIDING PAHR INITIAL CONDITIONS.

TIC
197 JACKSON, MAMES F. (LA-UR

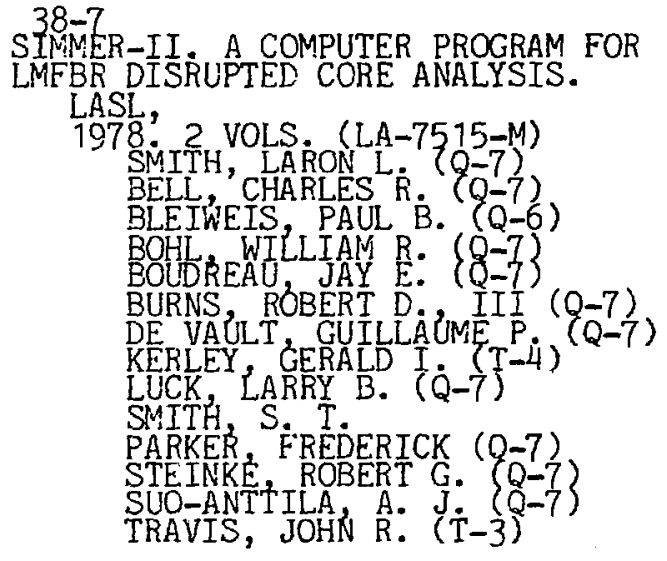

38-8

SIMPLE COMPUTATIONAL MODEL FOR THE PREDICTION OF FUEL PIN FAILURE IN THE TRANSIENT OVERPOWER ACCIDENT.

TRANS. AM. NUCL SOC.,

V.28, P. 474-5. 1978.

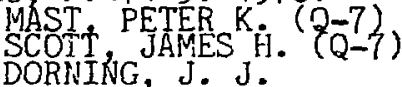

DORNING, J. J.

38-9

STATISTICAL ANALYSIS OF SIMMER-II

RESULTS

TRANS. AM. NUCL SOC., BURNS, ROBERT D.
LUCK, LARRY BI $(Q-7)$

$38-10$

TWO - PHASE MOMENTUM EXCHANGE EXPERIMENT AND ANALYSIS.

TRANS. AM NUCL. SOC.,

V.28, P. 445-6. 1978 . REXROTH, PAUUL E. (Q-7)

STARKOVICH, VICTOR S. (Q-8) 
CÅLCU

CALCULATIONS OF THE EFFECTS OF

INCOMPLETE PRETONIZATION IN

HIGH VOLTAGE THETA PINCHES.

PHYS. FLUTDS,
V.21 P. 1410-6. 1978;
SGRO, ANTHONY 6 . (CTR-6)

CLิด-2 2 IFICATION OF BE I-LIKE AND

B IELTKE IRON AND VANADIUM

$$
\begin{aligned}
& \text { J. } 68 \text { P P. } 48-51 \text {. } 1998 . \\
& \text { BROMAGE, G. E. } \\
& \text { COWAN ROBERT D. } \\
& \text { FAWCETT, B. } \dot{A} \text {. }
\end{aligned}
$$

\section{cón-3}

THERMONUCLEAR NEUTRONICS DATA.

LASL,

1978. 235P. THESIS, GEORGIA

INST: CF TEECHNOLOGYे, ATLANTA, 1977.

(LA-7067-T)

REUPKE, WILLIAM! A. (T-2)

$39-4$

ENERGY - EALAICE AND ECONOMIC

CONSIDERATIONS FOR FUSION THERMOCHEMICAL

HYDROGEN PRODUCTION.

TRANS. AM. NUCL. SOC.,

V. $30, P .4 \dot{2}-3.1978$.

KRAKOWSKI; ROBERT A. (CTR-12)

$39-5$

EXPERIMENTAL AREA AND REMOTE

HANDLING FACILITIES OF THE JET

TARGET INTENSE NEUTRON SOURCE.

TIC 1978 . 17P. MN (LA-UR-78-1229)

MEIER, KARL L. $(\mathrm{P}-14)$

$39-6$

EXTRACTICN OF TRITIUM FROM HELIUM

STREAMS USING LANTHANUM 5.25 NICKEL. 1978: 9P. (LA-7209-MS) CARSTENS, DEAN H. W. (CMB-3)

3EAT TRANSFER IN THE LITHIUM - COOLED BLANKET OF A PULSED FUUSION REACTOR. TIC 1978 . 7P. MN (LA-UR-77-1754) CORT GEORGE E. ( $W X-4$ ) KRAKOOWSKI, ROBERT A. (CTR-DO)
$39-8$

HYDROGEN ISOTOPE DIST'ILLATION FOR THE TPITIUM SYSTEMS TEST ASSEMBLY. TIC

1978 . 7P. MN (LA-UR-78-1325) EARTLIT, JOHN R. $(\mathrm{Q}-10)$ SHERMAN, ROBERT $\mathrm{H}:\left(\begin{array}{c}Q-10 \\ 0-10\end{array}\right)$

$30-9$

LASL CONTROLLED THERMONUCLEAP.

RESEARCH PROGRAM, JARUARY - DECEMEER 1976 LASL, 1978, 220P. (LA-7082-PR)

$39-10$

MEASUREMENT OF NON - MAXWELLIAN ELECTRON VELOCITY DISTRIBUTIONS IN A REFLEX

SCHARGE

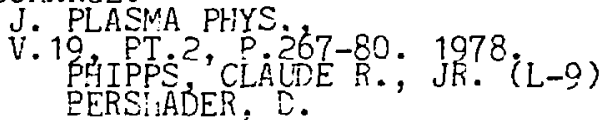

MULTIDIMENSIONAI QUASINEUTRAL PLASMA STMULATION VOLEL.

V. 29 COMPUT PHYS. 1978.

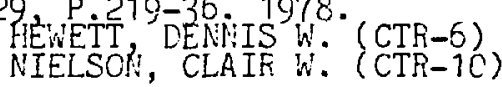

$39-12$

NOVEL INTERFEROMETER FCR THE

MEASLREMENT OF FLASMA DEISITY.

REV. SCI. INSTRU:

V. 49 P. $318-20.1978$. JACCBSCN, ABRAM $R$ : (CTR-?)

CALL, D.' L. $(L-10)$

$39-13$

NUCLEAR MATTER DISTRIBUTICNS NITH COMMENTS NUCL. PART.

PHYS., 1978, .8 , 1:. 3, P. $75-88$. JOHNSON MIKKEL E.
EETHE, HANS $A .(T-D O)$

$39-14$

PRCPOSAL FOR THE THEORETICAL

INVESTIGATION OF THE RELATIVISTIC

BEAM-PLASMA INTERACTION WITH

APPLICATION TO THE PROOF-OF-

PRINCIPLE ELECTRON BEAM-HEATED

LINEAR SOLENOIDAL REACTOR.

LASL, 18P. (LA-7359-P)
1978 THODE, LESTER E. (T-15) 
39-15

RECENT W'ORK ON NORMAL AMD

SUPERCONEUCTING INDUCTIVE ENERGY

STORAGE AND SWITCHING AT THE

EFREMOV INSTITUTE, LENINGIRAD,

USSR.

ENGINEERING PROBLEMS OF FUSION

KESEARCH. 1977 . SYMP. 7TH,

P.907-8. M.S. LUBELL' ED.

IEEE, 1977 (537/ S9898N 1977)

ALSO PUBLISHED IN :

TIC

1977. 3P. M: (LA-UR-77-2390)

MACHALEK, MILTON D. (CTR-7)

$39-16$

SECOND - ORDEF THEORY FOR

K PARALLEL TO B O ELECTROMAGINETIC

INSTABILITIES.

PHYS. FLUIDS,

V.21 P. $92-80.1978$.

GAPY S. PETER $(\dot{P}-4)(P-4)$

39-17

SHIELDING CALCULATIOHS FOR THE

INTENSE NEUTROH SOURCE FACILITY -

FINAL REPORT.

LASL,

1978: 19P. (LA-7332-MS)

BATTAT VORRIS E. $(T-1)$

HENAINGER, RUDOLPH $\mathrm{J} .(\mathrm{T}-1)$

MAC DONALE, JAMES L: $(T-1)$
DUDZIAK, DONALE J. $(T-1)$

$39-18$

STIMULATED BFILLOUIN BACKSCATTER

DETECTION FROM A THETA - PINCH PLASMA. V. APFL. PIYS. ARMSTRONG, WILLIAN T. (CTR-3)

SITDY OF EQUILIERIUN AIID STAEILITY OF

HICH-BETA TCKAMAKS SURROUNCED

BY FORCE-FREE FIELDS.

STICHTING VOCR FUINDAMENTEEL

ONDERZOEK DER MATERIE, JUTPHAAS

(NETHERLANDS). IIISTITUUT VOOR

PLASMA-FYSICA. RIJNEUIZEN REPOFT

78-108. 120P 1978 .

D'LPPOLTTO, D. A.

GOEDBLOED; J. P.

REV, J.

SYSTEM OF NOILINEAR PARTIAL DIFFERENTIAL EQUATIONS DESCRIBING CYLINDRICAL PLASMA COLLAPSE.

1978: 28P. (LA-6824)

CARASSO, ALFRED S.
SUYDAM, BERGEN R. (T-7)
$39-21$

TECHHOLOCY PROBLEMS IN THETA PIHCH REACTORS.

TIC THOHASSEN, KEITH I. (CTR-DO)

$39-22$

THREE - DIMENSIONAL MAGNETOHYDRODYNAMIC TUREULENCE IH CYLINDRICAL GEOMETRY. PHYS. FLUIDS

V. $21 ;$ P $757-64.1978$. MONTGOMERY DAVID TURNER, LEAF (CTR-6) VAYIALA; GEORGE

$32-23$

TRIDENT-CTR. A TWO-DIMENSIONAL TRANSPORT CODE FOR CTR APPLICATIONS. TIC 197 SESP. MN (LA-UR-78-1288)

$39-24$

TRITIUM SYSTEMS TEST ASSEMRLY AT THE LOS ALAMOS SCIENT IF IC LABORATORY. TIC

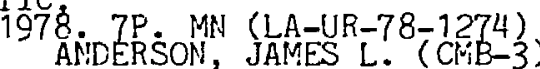

USE OF BINAFY ALLOYS OF THE LANTHAIIDES FOR TRITIUMA RECOVERY. TRANS. AM. PUCL. SOC., V. $28, P .202$. 1978 .

ALSO PUBLISHIED IN:

TIC

1978 . 5P. MN (LA-UR-78-1231) CARSTENS, DEAN H.W. (CMB-3)

VACUUM INTERRUPTERS USED FCR THE

INTERRUPTION OF HIGH DC CURRENTS, ENGINEERING PROBLEMS OF FUSION RESEARCH. 1977. SYMP. 7TH, KNOXVILLE, TR. PROC.; V.2, P. $1774-8$. M.S. LUEELL, ED' IEEE, 1977 ( $537 /$ S989EN 1977) ALSO PUBLISHED IN:

TIC,
1977 GP, WN (LA-UR-77-2356)
WARRN, ROGER W. CTR-9)

$27-40$ ADMINISTRATIVE PLAN. LASL, 20P. (LA-7425-MS) THOMAS, KEITH S. (CTR-7) COMP.

$27-40$ TOROIDAL REVERSED - FIELD

PINCH.

IASL

MINI-REVIEW, 1978. 4P.

(LASL-78-18) MILTON D. (AT-4) 
40. MATERIALS

40-1

ABSORPTION OF DEUTERIUM BY CERIUM CONTAINING BINARY ALLOYS.

J. NUCL. MATER .

V.73 PARTENS, DEÂN H. W. (CMR-3)

\section{0-2 - GAMMA PHASE TRANSITIONS IN \\ CERIUM - THORIUM ALLOYS UNDER HIGH PRESSURE.}

TIC 267 MN (LA-UR-77-2153)

ALSO PUELISHED IN:

HIGH PRESSURE AND LOW TEMPERATURE

PHYSICS. 1O77. INTRNATIONAL

CONEERENCE CLEVELAND. PROC.,

P. 169-86. C. W. CHAN, ED.

PLENUM 1978

HUANG, CHAO-YUAN $(Q-10)$

SMITH, JAMES L. ( CMB-5)

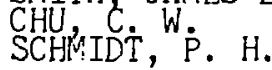

40-3

TO ION SCATTERING FPOM SOME

ACT INIDE MATERIALS

J. VAC. SCI. TECHNOL.,

$\mathrm{V} 15$, P. $679-83$. 1978 .

ALSO'PUBLISHED IN:

TIC,

23P. MN (LA-UR-77-2O72)
ELIIS, WALTON P. (CME-8)
TAYLOR, THOMAS N. (CME-8)

40-4

BRAZ ING MOLYBDENUM AND TLNGSTEN FOR

HIGH TEMPERATURE SERVICE.

WELDING J. RES.

$$
\begin{aligned}
& \text { LUNDBEERG, LYNN B. }(Q-13) \\
& \text { TURNER, WILLIAM C. }(1 .-10) \\
& \text { HOFFMAN, CARRCLL G. }(L-7)
\end{aligned}
$$

$40-5$

BRITTLE FRACTURE IN IRIDIUM.

$$
\begin{aligned}
& \text { METAIL. TRANS. } \\
& \text { V. } 9 A \text { P } 481-8.1978 . \\
& \text { HECKER, SIEGFRIED S. (CMB-5) } \\
& \text { ROHR, DANA L. (CMB-5) } \\
& \text { STEIN, D. F. }
\end{aligned}
$$

$40-6$

CERAMIC MATERIALS APPLICATIONS IN FUSION

REACTORS.

CERAM: IND,

V. 110, NO. 4,4 PAGES. 1978.

HURLEY, GEORGE F. (CMB-5)
40-7
CESIUM SOLUBILITY, DIFFUSION,
AI!D PERMEATION IN'ZIRCONIUM CAREIDE.

$$
\begin{aligned}
& \text { J. NUCL. MATER. ' } \\
& \text { V.73 P } 169-79.978 . \\
& \text { STAFK, WALTER A., JR. (CME-3) }
\end{aligned}
$$

40-8

COMPARISON OF INITIAL DAMAGE RATES

USING NEUTRON AND ELECTRON

IRRADIATIONS.

LASL

1978, THESIS, STATE U. OF NY,

STONY BFOOK, 1978. 137P.

GOLDSTONE, JOYCE A. ( CMB-8)

$40-9$

DAMAGE ENERGY FUNCTIONS FOF COMPOUNDS AND ALLOYS.

TRANS. AM. NUCL. SOC.,

ALSO' PUELISHED IN:

TIC77. 6P. MN (LA-UR-77-1444)

PARKIN, DON

COULTER, C. ALTCN (MP-DO)

40-10

DENSITY OF STATES CF ECC

NICEIUM (3) GOLD - A CCMPARISON WITH

A-15 NIOEIUM(3) SOLD.

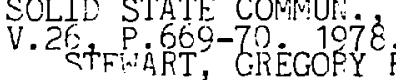

GIORCI, AREC

40-11

DIFF ICULTIES III PRODLCIIG PURE

ALPHA - PHASE PLUTOR:IUH.

J. NUCL. MATER.

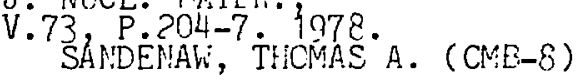

$40-12$

DYNAMIC PLASTIC INSTAEILITIES IN

STRETCHING PLATES AND SHELLS.

$$
\begin{aligned}
& \text { J. } 45 \text { PPP. MECH' } 105-10 \% \text { ' } 1978 \\
& \text { TAYLOR, JOHN W. (N-DO) } \\
& \text { HARLON, FRAICIS H., JR. (T-3) } \\
& \text { AMSLEN, AMTHONY A.' (T-3) }
\end{aligned}
$$


$40-13$

FABRICATION OF AN ALUMINA TORUS FOR THERMONUCLEAR FUSION

CONTAIMMENT.

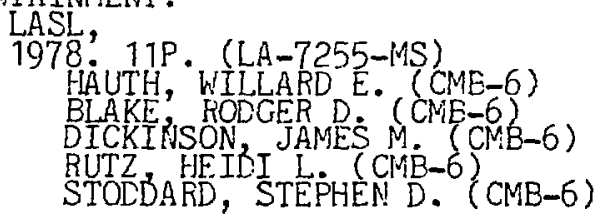

$40-14$

HANDBOOK OF FTLLERS ALD

REINEORCEMENTS FOR PLASTICS.

VAN NOSTRAND REINHOLD

1978. 652P. (668.4/K19H)

KATZ, HARRY S.
MILEWSKI, JOHN V.

ED.

ED.

4-. 15

HIGH CONDUCTIVITY, HIGH SPEC IF IC

IIEAT COPPER FOR CRYOGENIC

APPLICATIONS.

J. AQPL. PHYS. STEYERT, WLLLIAM $A$. JR. (0-10)

$40-16$

HICH FIELD PROPERTIES OF

CVD-PREPARED MIORIUM(3)GERMANIUM.

EULL. AM, PHYS, SOC.,

V.23, P. 322.1978

THOMPSOH JOE D. $(Q-10)$

MALEY MARTIN P: $(Q-10)$

NE'KIRK, LAWREICE R. (CMB-3)

40-17

INJECTION MOLDAELE CEFAMICS.

1978: 10P. (LA-6960)

NEWFIELL STEPHEN E. (CMB-6)
GAC, FRAIIK D. (CME-6)

4C-18

INVESTICATION OF COMPOSITES

W'TILIZING LOW - COST SMALI

DIAMETER SPHERES AS A FILLER.

REINFORCED PLASTICS COMPOSITES

INSTITUTE. TECHNICAL CONE 1978.

33RD ANNUAL, WASHTNGTON, $\dot{D} . C$.

PROC., SECT'2-A, P.T-5.' SPI,

1978 .

EHRENREICH, LEO C.

MATZ, HARRY S, JOHI $\mathrm{V}$.

40-19

ION - SCATTER ING STRUCTURE STUDIES

OF URANIUM DIOXIDE SIJREACES.

SURF: SCI $75.279-86.1978$.

ELLIS, WALTON $\mathrm{P}$ (CMB-8)
$40-20$

IT INERANT ANT IFERROMAGNETISM

OF TIBE (2) -

LETT V':39, P.L-441-2. 1978.

MATTHIAS, B. T.

GIORGI ANGELO L. (CME-3)

STRUEB NNG VERNON O (CMB-5)
SMITH, JAMES L. (CMB-5)

40-21

LEED STUDY OF URANIUM

OXIDE (APPROXIMATELY 100) VICINAL

SURFACES.

SURF. SCI.

V. $\left.77^{\circ}, \mathrm{P} \cdot 3 \dot{2}\right\}-36.1978$. TAYLOR, THOMAS N. (CMB-8)

ELLIS, WALTON P. (CME-8)

$40-22$

MAGNESIUM OXIDE AND SPINEL PARTS

FABRICATION.

LASL

1978: $5 P$ (LA-7289)

SHEINBEKG, HASKELL (CMB-6)

GAC FRALK D. (CMB-6)

HAUTH, WILLARD E. (CME-6)

40-23

MAGNETIC AND SUPERCONDUCTING

TRANSITIONS IN GD $(X) E R(1-X) R H(4) B(4)$

AND GD $(X) Y(1-X) R H(4) B(4)$.

1977. 11P. MN (LA-UR-77-2372)

ALSO PUELISHED IN:

J. APPL. PHYS,

V. 49 P. 1392-4' 1978. WANG RUN-HAN (Q-10)

LASKO'WSKI R. J. $(Q-10)$

HUANG, CHAO-YUAN $(Q-10\}$

SMITH:, JAMES L. (CMB-5)

CHU, C. W. (Q-io)

40-24

MELTING POINT OF YTTRIA.

HIGH TEMP. SCI.' 1977. SKAGGS, SAMUEL R. (CMB-3)

METAL - CAREON BOND DISSOCIATION

ENERGIES AND ENTHALPIES OF

FORMATION FOR GASEOUS METAL

CARBONYLS

J: IESS-COMMON METALS,

58 BEHRENS, ROBERT
G. (CMB-3)

$40-26$

MICROWAVE RESISTIVITIES OF TM/SUB Y/ TMA SYU Y SUB $1-Y$ Y $/ S$ AND $1-Y / T E$.

V. 41, PHY . SOC. JAPAN, 19076. SUANG CHAO-YUAN (Q-26) 
40-27

PHASE INVESTIGATIONS OF THE PLUTONIUM AND PLATINUM PLUTONIUM RHODIUM, SYND PMS

J. NUCL. MATER.

V.75. P. $262-73$ :' 1978

LAND, CLETIS C. (CMB-5)

PETERSON, DEAN E (CME-5)

ROOF, RAYMOND BRADLEY, JR. ( CMB-5)

$40-28$

POINT DEFECT DISLOCATION SINK STRENGTH.

J. NUCL. MATER. 3978.

GREEN, WALTER V. (CMR-8)

WEERTMAN, JOHANNES (CMB-8)

$40-29$ PROCESSESS OF PLASTIC DEFORMATION, PROPERTIES OF THE F INAL PRCDUCT. TRANSLATION OF N'EKOTORYE STORONY PROBLEMY POLUCHENIIA

SVERKHPROVODIASHCHIKH MATER IALOV BY

I. M. PAVLOV ET AL. PLASTICHESKAIA

DEFORMATISIIA NERIADNOVYKH

METALLICHESKIKH MATER IALOV,

P. 184-92. 1976.

LASL,

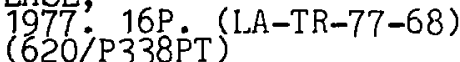

MANCA, VAFLERIE M. (ISD-4) TRANSLATOR

40-30

QUANTITATIVE COMPARISON OF THE

DOUBLY INTEGRATED KLL AUGER

SPECTRA OF MAGNESIUM, ALUMINUM

AND SILICON WITH THEIR OXIDES.

PROGRESS IN QUANTITATIVE SURFACE

ANALYSIS. 1977 SEYMP SOANTITATIVE

SURFACE ANALYSIS OF MATERIALS.

N.S. MCINTYRE ED. P.64-71. ASTM,

SPRINGER, ROBERT W. (CMB-6)

GAAS T. ${ }_{\text {GRANT }}{ }^{\mathrm{T}} \mathrm{T}$.

40-31

RATE AND PRESSURE DEPENDENCE OF

CONTAMINANTS IN VACUUM DEPOSITED

ALUMINUM FILMS.

J. VAC SCI. TECHNOL.,

V. $15, P .210-4.1978$.

SARINGER, ROBERT W (CMB-6)

$40-32$

RE-ENTRANT SUPERCONDUCTIVITY AND

MAGNETIC TRANSITIONS IN TMX ER1-X RH4B4.

BULL. AM PHYS. SOC.,

V. 23 SMITH, JAMES 19

ROOF, RAYMOND BRADLEY, JR. (CMB-5)

STRUEBING, VERNON 0. (CMB-5)
40-33

REPLY TO A CRITICISM OF A PAPER REGARDING MARTENSITIC BEHAVIOR OF ALPHA-PHASE URANIUUM.

SCRIPTA MET. ' 1978 SAMDENAW; THOMAS A. (CME-8)

40-34

RESOLUTION FACTORS IN THE USE OF A DOUBLE - PASS CMA FOR ISS.

J. VACUUM SCI. TECH.,

$\mathrm{V} \cdot 15, \mathrm{P} \cdot 1769-70.1978$ ELLIS, WALTON P ( $M$ ME-8)

$40-35$

REVIEW OF SOME PAST AND

PRESENT POWDER METALLUFGY

PROGRAMS AT THE LOS ALAMOS

SCIENTIE IC LABORATORY.

TIC77. 38P. NN (LA-UR-77-719) SHEINBERG, HASKELL (CME-6)

$40-36$

SOME CORFOSION TESTS OF MATERIALS

IN! UFANIUM HEXAFLLIORIDE.

LASL,

1973̈. 19P. (LA-7327-MS)

FLOORIN, ALAN E.

40-37

SOME MICFOSTRUCTURES DEVELOPED IM 303 STAILLESS STEEL WHICH HAS DEEN SHOCK LOADED TO THE MECABAF RANGE. T977. 14P. M! (LA-UR-77-1875) SANDSTROM, DONALD J. (CME-6)
JONES, THOH:AS I., JR. (CME-6)

40-38

SPECIFIC HEAT OF $A-15$ AND BCC MOLYEDENLM $(0.4)$ TECHMETIUM $(0.6)$. PHYS REV.

V. 17B, P: 3534-40. 1978. STEWAET, GREGORY R ( $(\mathrm{CMB}-3)$
GIORGI, ANGELO L. (CME-3)

40-39

SPECIFIC HEAT OF SINGLE PHASE

NIOBIUM (3) GERMANIUM.

SOLID STATE COMMUN

V. 26 P P $417-20$ - 1978 . SEWAR, GREGOR, $(\mathrm{CM} B-3)$ NEWKIRK, LAWRENCE R. ( $\mathrm{CME}-3)$

$40-40$

SPECIFIC HEAT OF YTTRIUM/SUB $0.7 /$

THORIUM/SUE 0.3/CARBON/SUB 1.58\%.

SCLID STATE COMMUN.

V. 27, P. $413-6.1978$. STEWART, GREGORY R, (CMB-3) GIORGI, ANGELO L. $\left\{\begin{array}{c}\text { CMB-3 } \\ \text { KRUPKA, NILTON C. } \\ \text { CME-3 }\end{array}\right\}$ 


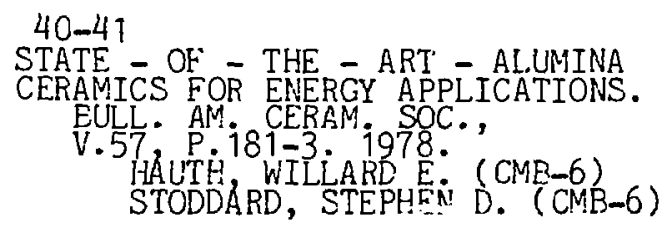

$40-43$

STRUCTURE AND MECHANICAL PROPERTIES

OF ALUMINLM - ALUMIMUM $(X) O X Y G E N(Y)$

VACUUM DEPOSITED LAMINATES.

IIC 1978 . 26F. MN (LA-UR-78-384)

SPRINGER, ROBEKT W, (CME-6)

CATLETT, DUANE S. (CNB-6)

$40-44$

SUPERCONDUCTIVITY OF AMERICIUM.

SCIEHCE

SMITH, JAMES L. (CMB-5)

HAIRE, RICHARD $G$.

$40-45$

TEIEORETICAL STUDY OF THE EFFECT

OF PRESSURE ON DIFFUSION IN

ALUMINUM.

J. NUCL. NATER

V. 69 \& 70, P. $528-32.1978$.

$40-46$

UNUSUAL SUPERCONDUCTING BEHAVIOR

OF THE MOLYDDENUM - TECHNETIUM

SYSTEM

PHYS. REV. $1760-2.1978$.

GIORCI ANCELO L. (CNE-3)

MATTHIAS, E. T.

$40-47$

VARIABLE LOW - TEMPERATURE MARTENSITIC

BEHAVIOR OF ALPHA - PHASE URANIUM.

SCRIPTA MET 11.1978.

SANDENAW, THOMAS A. (CMB-8)

$40-48$

WIISKERS AND MICROFIBERS.

ADDITIVES FOR PLASTICS.

R. E. SEYMOUR ED. V.1. P $79-122$.

ACADEMIC, 1978 J $(668.4 /$ A227P $)$ 
41. MATHEMATICS AND COMPUTERS

\section{'SENISING' THE ELLIPTICAL TORUS. \\ LASL, 6P. (LA-7187-MS) CASHWELL, EDMOND D. (TD-6)}

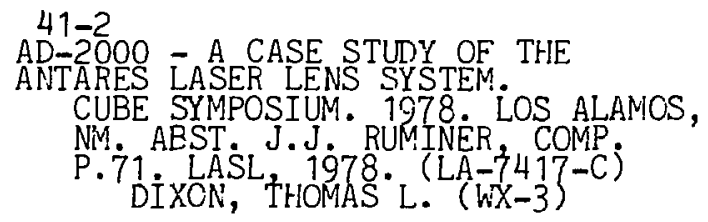

\section{1-3}

APPLICATION OF ARTIFICIAL INTELLIGENCE TECHNIQUES TO THE ACCELERATION OF MONTE CARLO TRANSPORT CALCULATIONS. 19A8L: 71P. (LA-7475-MS) MAC DONALD, JAMES L. (T-1)
CASHWELL, EDMOND D. (TD-6)

$41-4$

APPLICATION OF COMPUTED TOMOGRAPHY BOUNDARY DETECTION, AND SHADED GRAPHICS RECONSTRUCTION TO INDUSTRIAL INSPECTION.

MATER. EVAL. 6 PACES, 1978. KAUGER, RTCHARD (M-Z

$41-5$

APPLICATICN OF COMPUTERS AND GRAPHICS TO PHYSICAL INSPECTION

PROBLEMS.

CUBE SYMPOSIUM. 1928. LOS ALAMOS, NM. ABST. J.J. RUMINER, COMP. P.72-3. LA SL, 1978 PENTECOST, BENLEE A. (LX-317-C)

41-6

BAYESIAN MODEL FOR DETERMINING THE OPTIMAL TEST STRESS FOR A SINGLE TEST UNIT. TE.CHNOMETR ICS

V.20 P.179-85. 1978. MARTZ HARRY F JR. WATERMAN, MICHAÉL S: (2-12)

$41-7$

CAD-CAM INTERACTIVE GRAPHICS SYSTEM DESICNED BY USERS. TIC 200 . MN (LA-UR-78-179)
LAUER, DONALD J. (WX-3)
CAMAC INTERFACE MODULE FOR PACE ADC SYSTEM. METHODS, NUCL INSTRUN, METHODS, DALTCN CHARLIE G: (MP-4) MISCHKE, RICHARD E. (MP-4) SCOTT, D.T.

41-9

CERTIFICATION REPORT ON 'EFFICIENT FORTRAN SUEPROCRAMS FOR THE SOLUTION OF ELLIPTIC PARTIAL DIFFERENTIAL EQUATIONS' BY PAUL SWARZTRAUBER AND ROLAND SWEET NATIONAL CENTER FOR ATMOSPHERIC RESEARCH, EOULDER, COLORADO. LASL, 1978. 17P. (LA-7524-MS) STEUERWALT, MICHAEL (C-3)

41-10

CHARACTERISTICS OF COMPUTED TOMOGRAPHIC RECONSTRUCTION NOISE AND THIEIR EFFECT ON: DETECTABILITY.

TIC, 97 4P. MN (LA-UR-77-2409)
HANSON, KENNETH M. (MP-3)
BOYD, D. P.

41-11

CLASS OF NEAR-PERFFCT CODED APERTURES. TIC, $7 P$ M M (LA-UR-77-2369)
CANNOA THOMAS M- $(M-8)$
FENIMORE, EDWARD $(P-4)$

$41-12$

CODED APERTURE IMAGINC WITH UNIFCRMLY REDUNDANT AFRAYS.

APPL 17 PPT $332-47,1978$ FENIMORE, EDWARD (P-4) CANNON, THOMAS M. $(M-8)$

$41-13$

COMIVENTS ON "NONSTATIONARY ASSUMPTIONS

FOR GAUSSIAN MODELS IN IMACES." IEEE TRANS. SYST MAN CYBERN., V.SMC-8, P. 579-82. 1973 TRUSSELL HENRY $\mathrm{J}(\dot{\mathrm{N}}-3)$
KRUGER, RICHARD $(\mathrm{M}-\mathrm{B})$ 
41-14

COMMON FILE SYSTEM

SPECIF ICATIONS,
SEPTEMBER 1977 .

LASL, 24P. (LA-7065-MS)

ALSO PUBLISHED IN:

TIC , 28P. MN (LA-7065-MS)

BLOOD, PHILIP M. (C-4)

CHIRISTMAN, RONALD D. (C-4)

COLLINS, WAURICE W. $(C-4)$

COMPARISON OF IMAGE

RESTORATION METHODS.

APPL. OPT.

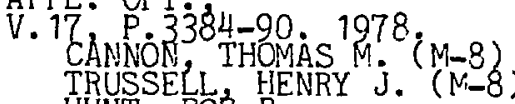

HUNT, BOB $\mathrm{R}$.

41-16

COMPUTER - GENERATED MOVIES AS

AN ANALYTIC TOOL.

TIC, 678. $6 \mathrm{P}$. MN (LA-UR-78-66)

ELLIOTT, RAYVIOND L. (C-6)

COMPUTER ALGEBRA AND ITS APPLICATIONS.

CUBE SYMPOSIUM. 1978 . LOS ALAMCS,

NM. ABST. J.J FUMINER, COMP.

P.68. LASL, 1978 . (LA-7417.-C) HOWELL, JO ANN $(\mathrm{C}-3)$

41-18

COMPUTER APPLICATIONS ANNUAL REPORT

JULY 1, 1976-SEPTEMBER 30, 1977.

LASL,

1978. 24P. (LA-7213-PR)

SANDERS, WILLIAN MORT $(H-6)$

LESTER, JEAN $V$.

CAMPBELL, CALVIN L

SMITH, WILLIAM ROSS, JR.

TAYLOR, PAUL $M$

WEATHINGTON CARL

PETERSEN, PAMELA M. $(\mathrm{H}-6)$

PAYNE, ROBERT J. $(\mathrm{H}-6)$

41-19

COMPUTER APPLICATIONS IN THE

ENGINEERING DESIGN OF THE W8O

WAREEAD.

CUBE SYMPOSIUM. 1973. LOS ALAMOS,

NM. ABST. J.J. RUMINER, COMP.

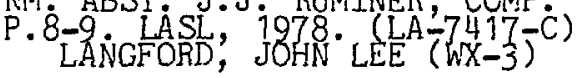

$41-20$

COMPUTER GRAPHICS - AN ESSENTIAL COMMUNICATIONS TOOL

CUBE SYMPOSIUM. 1978. LOS ALAMOS,

MM. ABST. J J. RUMINER, COMP.

KRUGER, RICHARD (M-8)

CANNON, THOMAS M, (M-8)

LUNDY' ARVID S. $(E-5)$

41-21

COMPUTER GRAPHICS FOR EXTRACTING INFORMATION FROM DATA.

TIC.
1978.

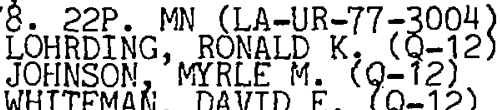

JOHNSON
WHITEMAY,, DAVLE M. $(Q-12)$
E $(Q-12)$

41-22

COMPUTER MODELING OF SCALE FORMATION IN GEOTHERMAL FLUID HEAT EXCHANGE EQUIPMENT

CUBE SYMPOSIUM. 1978. LOS ALAMOS,

NM. ABST. J.J. RUMINER, COMP

P. i02-3. LASL 1978. ( [A-7417-C)

MERSON, THOMAS J. (WX-4)

FEBER, ROY C. JR. (CME-8) PARKINSON, WILLIAM J. (WX-4) BACA, G. A.

41-23

COMPUTER SCIENCE AND SERVICES

DIVISION ACTIVITIES AND PLANS.

1978: 26P (LA-7093)

DORR, FRED $W$. (C-DO)

41-24

COMPUTER USE FOR PREDICTING

TORNADO-INDUCED FLOW AND

RE-ENTRAINMENT IN NUCLEAR

FACILITIES.

CUBE SYMPOSIUM. 1978. LOS ALAMOS,

NM. ABST. J.J. RUMINER, COMP.

P.65. LASL, 1978. (LA-7417-C)

ANDRAE, RICHARD W. (WX-8)

DUERRE; KENNETH H: $(W X-8)$

GREGORY, WILLIAM S. $(W X-8)$

MARTIN, RICPARD A. (WX-8)

41-25

COMPUTING AT LASL IN THE 1940"S

AND 1950 "S.

1978: 26P. (LA-6943-H)

VAZARUS ROGER B. (C-3)

WELIS MARK B. (T-7) (C-DO) 
41-26

CONDITIONED RANDOIM WALK WITH

APPLICATIONS.

LASL, 18P. (LA-6990-MS )
1978.
BEYER, WILLIAM A. (T-7)
WATERMAN, MICHAEL S. (Q-12)

$41-27$

CONTROLLING A FAST SOLID-STATE

CAMERA WITH A MICROCOMPUTEP

CUBE SYMPOSIUM. 1978. LOS ALAMOS,

NM. ABST. J.J. RUMINER, COMP

P.22. LASL, 1978 . (LA-74 $417-\mathrm{C})$

$41-28$

CRASHC - A TWO-DIMENSIONAL CODE

TO COMPUTE THE RESPONSE OF

AXISYMMETRIC SHIPPING

CONTAINERS TO END-ON IMPACTS.

CUBE SYMPOSIUM. 1978 . LOS ALAMOS,

NM. ABST. J.J. RUMINER COMP.

P.96-7. LASL, 1978. (LA-7417-C)

BUTLER, THOMAS A $(w X-8)$

ENDEBROCK, ELTON G. (WX-8)

41-29

ERY -1 ERROR RECOVERY.

VECTOR AND PARALLEL PROCESSORS.

1978. WORKSHOP LOS ALAMOS, NM. B.L.

BUZBEE, COMP. PROC., P. 11. LASL,

1978. (LA-7491-C)

MARUSAK, ALEX L. (C-3)

CRITICAL INDICES FROM PERTURBATION

ANALYSIS OF THE CALLAN-SYMANZIK

EQUATION.

PHYS. REV.

V. $17 \dot{B}, \stackrel{P}{P} 1365-74.1978$.

BAKER, GEORGE'A., JR. (T-11)

NICKEL, BERNIE G'

${ }^{41-31}$ CUBE (COMPUTER USE BY ENGINEERS)

SYMPOSIUM ABSTRACTS. OCTOBER 4-6, 1978.

LOS ALAMOS SCIENTIFIC LABORATORY

LOS ALAMOS, NM.

LASL,

1978, 103P. (LA-7417-C)

CUBE 11 SYMPOSIUM PROGRAM, OCTOBER 4-6, 1978 LOS ALAMOS SCIENTIFIC

LABBORATORY.

1978: 38P. (LASL-78-29)

LOS ALAMOS SCIENTIF IC LABORATORY
41-33

DAISY - A DATA ACQUISITION AND

IO CONTROL SYSTEM.

CUBE SYMPOSIUM: 1978. LOS ALAMOS,

NM. ABST. J.J. RUMINER, COMP. P. 59. LASL, 1978. (LA-7417-C) ROBINSON, JOEEL H. $(E-5)$ SCHNEIDER, J. DAVID $(P-14)$

BARNETT, WILLIAM (E-5)

$41-34$

DATA MANAGEMENT AND HANDLING FOR THE HYDROGEOCHEM ICAL AND STREAM SEDIMENT
RECCONNA ISSANCE PROGRAM AT THE

LOS ALAMOS SCIENTIFIC LABORATORY. LASL

1978̈: 3P. (LA-7128-MS)

CHEADLE, JESSE M. (C-5)

DEBUGGING AIDS

CUBE SYMPOSIUM. 1978. LOS ALAMOS,

NM. ADST, J.J. RUMINER COMP:

P. 66. LASL, $1978 . \quad(L A-7417-C)$
KAZEK, CHESTER S., JR. (C-5)

41-36

DEMOS USER'S GUIDE.

LASL, PAGED IN SECTIONS,
(LA 8-5525-M, VOL.7, REV.)
C-2 GROUP

41-37

DYNAMIC COMPUTER SIMULATION OF $A$

TRITIUM SYSTEM TEST ASSEMBLY IN

SUPPORT OF FUSION REACTORS.

CUBE SYMPOSIUM. 1978 . LOS ALAMOS,

NM. ABST J.J. RUMINER COMP.
P.91-2 LASL, 1978. (LA $-7417-\mathrm{C})$
GRUNDMANI!, JACK G. (E-4)

41-38

ENERGY FLCW DIAGRAMS.

CUBE SYMPOSIUM. 1978 . LOS ALAMOS,

NM ${ }^{2}$ ABST J. J R RUMINER, COMP. KOENIG, DANIEL R. $(0-13)$

41-39

ESTIMATION OF PROBABILITY

DENSITIES BY EMPIRICAL DENSITY

FUNCTIONS

$$
\begin{aligned}
& \text { J. OPTIMIZATION THEORY AND APPL., } \\
& \text { V.24 P. } 5-27 \text { 1978. } \\
& \text { WATERMAN, MICHAEL S. (Q-12) }
\end{aligned}
$$


$41-40$

EXAMPLES OF MAXIMUM LIKELIHOOD SPATIAL FILTERING.

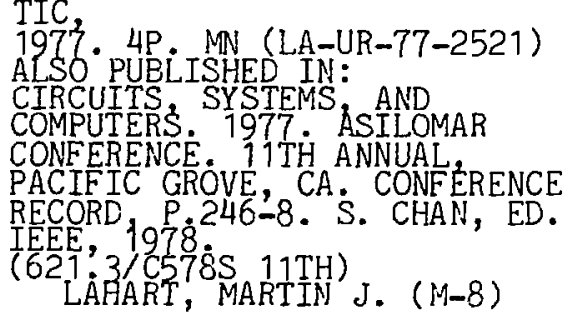

$41-41$

EXPERIENCES WITH FINITE ELEMENT

PROGRAMS ON THE CRAY-1 COMPUTER.

CUBE SYMPOSIUM. 1978. LOS ALAMOS,

NM. ABST J.J RUMINER, COMP OAKES WILLIAM R, JR, $(W X-3)$

$41-42$

FAST FOURIER TRANSFORM BY

POLYNOMIAL EVALUATION.
$J$. APPL. MATH. PHYS.

V.29 P. $387-94$. $1978^{\circ} \cdot(\mathrm{C}-3)$

$4 i-43$

FEASIBILITY STUDY FOR A POSTACCIDENT HEAT REMOVAL FACILITY.

\section{8}

978: 28P. (LA-7133-MS)

BARTS, EDWARD W. $(Q-7)$
APPERSON, COURTNEY E., JR. (Q-13)

DUNWOODY, WADE E. $(Q-13)$

BENNETT, JOEL G. $(Q-13)$

$41-44$

FINITE ELEMENT LINEAR MODELS FOR

REVOLUTION

CUBE SYMPOSIUM. 1978. LOS ALAMOS,

NM. AEST, J.J ROMINER COMP
P. 52 L LASL, 1978. (LA-7417-C)
COOK, WILLIAM A. (WX-8)

$41-45$

FIRE CONTROL SYSTEM.

CUBE SYMPOSIUM. 1978. LOS ALAMOS,

NM ABST J.J RUMINER COMP

$41-46$

FLOATING POINT HARDWARE EMULATOR

FOR RSX-11D.

T977. 5P. MN (LA-UR-77-2649)

KELLOGG, MARTIN P. (MP-1)

LONG, MARSHALL
41-47

FORECASTING METHODS FOR COMPUTER TECHNOLOGY.

1978. 18P. MN (LA-UR-78-1180) WORLTON, WILLIAM J. ( C-DO)

$41-48$

FORTRAN EXTENDED INTERACTIVE DEBUGGING AID.

LASL.

1978: 11P. (LA-7467-MS) KAZEK, CHESTER S., JR. (C-5)

$41-49$

FORTRAN 7 ?

COMMUN, ACM,
V.21, $806-20,1978$.
BRAINERD, WALTER S.

$\mathrm{ED}$

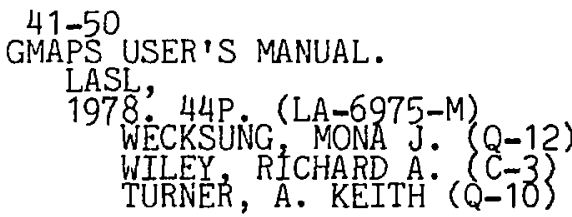

41-51

HIGH - PERFORMANCE GRAPHICS

SYSTEM FOR THE CRAY-1.

VECTOR AND PARALLEL PROCESSORS. 1978. WORKSHOP LOS ALAMOS, NM. B.L.

BUZBEE, COMP, PRO

EWALD, ROBERT H. (C-6)

MAAS, LYNN D. $(\dot{C}-6)$

$41-52$

HIGH PERFORMANCE GRAPHICS SYSTEM FOR THE CRAY-1.

1978. 7P. MN (LA-UR-78-1449) EWALD, ROBERT ${ }_{\text {MAAS, }}(\mathrm{C}-6)$

$41-53$

HYDRODYNAMIC CALCULATION OF JET

FORMATION BY HEMISPHERICAL - SHAPED CHARGES WITH TANTALUM LINERS. CUBE SYMPOSIUM. 1978. LOS ALAMOS, NM. ABST. J.J RUMINER COMP.

P. 12. LASL, 1978 (IA-7417-C) FORT GEORGE E. (WX-4

41-54

IMPLEMENTATION OF THE ACM/SIGGRAPH PROPOSED GRAPHICS STANDARD IN A MULTISYSTEM ENVIRONMENT. 1978 8. 5 MN (LA-UR-78-1427)
KELLNER, RICHARD G (C-6)
REED, THEODORE N. (C-6) 
41-55

INGEN. A GENERAL - PURPOSE MESH

GENERATOR FOR FINITE ELEMENT CODES. LASL, 58P. AND MICROFICHE: (LA-7 T35-MS) -
COOK, WILLIAM A. (Q-13)

$41-56$

INTERACTIVE GRAPHICS ON THE CRAY-1

SUPERCOMPUTER.

T1C78. 19P. MN (LA-UR-78-309)

MWALD, ROBERT $H .(C-6)$

$41-57$

INTRODUCTION TO Q.

LASL,

1978. 26P. (LA-7001-M)

KELLOGG, MARTIN P. $(M P-1)$

MINOR, MICHAEL M. (P-2)

SHLAER, SALLY

SPENCER, NANCY $C$.

THOMAS, RICHARD $\dot{F}$. JR.

VAN DER BEKEN, HENRI

$41-58$

K-TIF, A TWO - FLUID COMPUTER PROGRAM

FOR DÓWNCOMER FLOW DYNAMICS.

LASL,

1978: 130P. (LA-6994)

AMSDEN, ANTHONY A. $(\mathrm{T}-3)$

\section{$41-59$}

LAPU2. A LASER PULSE PROPAGATION

CODE WITH DIFF FACTION.

(2A-6955). AND MICROFICHE.

GOLDSTEIN JOHN C.: $\left.\begin{array}{l}\mathrm{L}-6 \\ \mathrm{~L}-5\end{array}\right\}$

\section{$41-60$}

LIBRARY OF PORTABLE MATHEMATICAL

\section{FUNCTIONS}

CUBE SYMPOSIUM. 1978. LCS ALAMOS,

NM. ABST. J.J RUMINER COMP.

P.66. LASL 1978. (LA-3417-C)

\section{1-61}

LINPACK WORKING NOTE NO 11 . LASL,

1978.' 18P. (LA-7389-MS) DONGARRA, J. J.

\section{1-62}

MAPPE

CUBE SYMPOSIUM. 1978. LOS ALAMOS,

NM. ABST. J.J. RUMINER, COMP.

P.94-5. LASL, 1978. (LA-7477-C)

DAHL, DAVID A. (H-8)
41-63

MASS STORAGE AT LASI

MASS STORAGE WORKSHOP. 1977. BOULDER, CO. PROC. K. D. FRIEDMAN, ED. P. $95-112$. NCAR, 1978 . $(621.381 / \mathrm{M} 414 \mathrm{~S}$ COLLLINS, MAURICE $w .(C-4)$

\section{1-64 \\ MAXIMUM ENTROPY ANALYSIS FOR SOME ONE- AND TWO - DIMENSIONAL PROBLEMS. 197

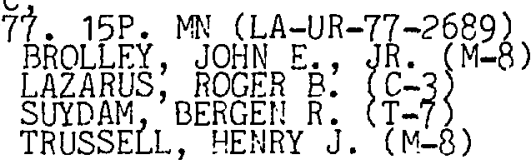

$41-65$

MAXIMUM LIKELIHOOD ESTIMATION

FOR THE BETA DISTRIBUT ION.

J. STATIST. C.OMPUT. SIMUL.,

V.7, P. $253-8.1978$.

BECKMAN, RICHARD J $(Q-1 \hat{Q})$
TIETJEN, GARY $(\dot{Q}-12)$

41-66

METHOD FOR ACCELERATING THE.

ITERATIVE SOLUTION OF A CLASS OF FREDHOLY INTEGRAL EQUATIONS.

$J$ OPTIMIZATION THEORY AND
APPL. V.24 P - -27 : 1978 .
ALIEN, RICHARD C (C-DIV)

ALLEN, RICHARD C (T (C-DI)

41-67

MICROPROCESSOR - CONTROLED CAMAC

DATA LINK MODULE.

LASL,

1978: 17P. AND APPEND.

$(L A-7299-M)$

POTTER, JERRY M. (MP-1)

41-68

MICROPROCESSOR CONTROLLED DUAL

PARAMETER ADC SYSTEM WITH A

CAMAC INTEREACE.

NUCL. INSTRUM. METHODS,

V. 154 P. $589-94.1978$. PERRY, LENIIS G. (MP-1) NICKELL, J. DONALD, JR. (E-5)

$41-69$

MICROPROCESSOR DATA - INPUT - CONTROL UN IT FOR MARK CENTURY NUUMERICAL CONTOURING CONTROL.

1978 ETHRIDCE, C. DWAYNE $(E-5)$
SEIFERT, $h I L L I A M$ M. $(E-5)$ 
41-70

MODELING THE MK 12A/W78

IMPULSE RESPONSE

CUBE SYMPOSIUM. 1978. LOS ALAMOS,

M. ABST. J.J RUMINER, COMP. SHAEER, BARRY P. $(W X-1)$

41-71

MONTE CARLO ASPECTS OF CONTRIBUTONS.

NUCL. SCI. ENG. 1978.

DUEI ARIE $(T-1)$

GERSTL, S. A. W' ${ }^{(T-1)}$

41-72

WEW PROBABILITY DISTRIBUTION WITH

APPLICATIONS IN MONTE CARLO STUDIES.

LASL,

1978: 10P. (LA-7095-MS)

JOHASON, MARK E $(0-12)$

41-73

NOTES ON LINEAR IMAGE

RESTORATION BY MAXIMIZING

THE A POSTERIORI PROBAABILITY. IEEE TRANS. ACOUST. SPEECH,

SIGNAL PROCESSING, V.ASSP- 26 ,

P. 174-5 1978 HENRY J. (M-8)

41-74

NUMERICAL SIMULATION OF THREE -

DIMENSIONAL FLOW PAST BLUFF BODIES. COMPUT. METHODS APPL. MECH.

ENG. V. $14, P$. 93-124: 1978 .

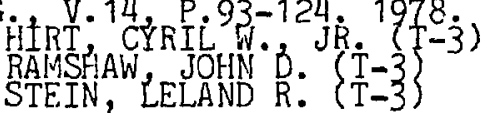

41-75

ON A CLASS OF LINKED

DIAGRAMS, I. ENUMERATION

J. COMEINATORIAL THEORY,

V.24 P. $\left.357-66 .{ }^{1978} \cdot 7\right)$

41-76

ON A CLASS OF LINKED DIAGRAMS.

II. ASYMPTOTICS

DISCRETE MATH.

V.21 P. 309-18', 1978

STEIN PAUL R. $(T-7)$

41-77

ON A QUADRATIC RECURRENCE RULE

F FALTUNG TYPE

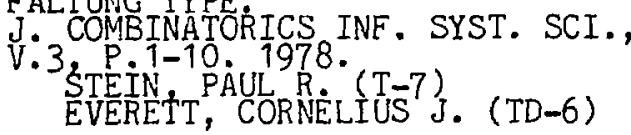

41-78

ON BLOCK RELAXATION TECHNIQUES.

WISCONSIN U. MADISCN

MATHEMATICS RES. CENTER.

TECH. SUMMARY REPT 10. 1860.

1978: (MRC-TSR-1860)

BOLEY, D. LUCIUS

BUZEEE, EILLY L. (C-3)

PARTER; S. V.

ON 1 CHERNOFF FACES.

TIC
1978 BRUCKNER, LAWRENCE A. (Q-12)

ON THHE LATTICE OF FACES OF THE

BULL. AM. MATH. SOC. ,

.84 P.284-6. 1978 .
METROPOLIS, NICHOLAS C. (T-7)
ROTA, GIAN-CARLO

ON THE STRUCTURE OF COMPUTER

CODES FOR SOLVING PARTIAL

DIFFERENTIAL EQUATIONS

CUBE SYMPOSIUM. 1978. LOS ALAMOS,

NM. ABST J.J. RUMINER COMP.

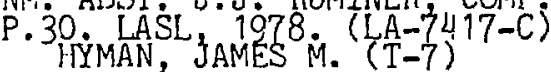

41-82

OVERVIEW OF CTR-DIVISION CONTROL

AND DATA ACQUISITION COMPUTER USAGE AT

THE LOS ALAMOS SCIENT IF IC LABORATORY.

CUBE SYMPOSIUM. 1978. LOS ALAMOS,

NM. ABST J.J. RUMINER COMP

CHANDLER, GEORGE I. (CTR-8)

WILLBERG, JOHN W. (CTR-8)

41-83

PARTITIONS INTO CHAINS OF A CLASS

OF PARTIALLY ORDERED SETS.

PROC. AM MATH. SOC.

MÉTROPOOLIS; NICHOLLAS C. (T-7)

ROTA, GIAN-CARLO

STREHL, VOLKER

WHITE, NEIL

41-84

PDP $11 / 03$ BASED, DUAL PARAMETER

DATA ACQUISITION SYSTEM.

DIGTTAL EQUTP

V.4 P. 59 i-5. 1977.

ALSSO PUB́LISHED IN!

TIC77. 6P. MN (LA-UR-77-2642)

GIESLER GREGG CARL (CNC-11) 
$41-85$

PEBBLE - A THERMAL - HYDRAULIC ANALYSIS CODE FOR AXISYMMETRIC PEBBLE BED NUCLEAR REACTOR CORES. CUBE SYMPOSIUM. 1978 . LOS ALAMOS, NM. ABST. J.J. RUMINER, COMP 1978 . (LA-7417-C) STROH, KENNÉTH R. $(Q-13)$

41-86

PERM, CORRECTIONS TO THE K-FIX CODE.

1978 , 4P (LA-NUREG-6623, SUPPL.)

RIVARD, WILLIAM C. (T-3)'
TORREY, MARTIN D. (T-3)

PIM-87. PROGRAMMER'S INFORMATION

MANUAL. GUIDE TO THE PROGRAM

LIBRARY AND ABSTRACTS.

1978: LOOSELEAF. (LA-5525-M, VOL.2) SOFTWARE DOCUMENTATION GROUP

\section{$41-88$}

PIM-3. PROGRAMMER'S INFORMATION

MAN'JAL. THE LASL FORTRAN

EXTLNDED REFERENCE MANUAL

LASL,

1978' LOOSELEAF. (LA-5525-M, VOL. 3) SOFTWARE DOCUMENTATION GROUP

FIM-49. PROGRAIMMER'S INFORMATION

MANUAL, LASL GUIDE TO NOS. LAS.,

1978. LOOSELEAF. (LA-5525-M, VOL.4) SOFTWARE DOCUMENTATION GROUP

\section{1-90}

PLI FORTRAN PROGRAMMING FOR

URANIUM DATA BASES AT LASL. 1977. 12P. MN (LA-UR-77-2023) CHEADLE, JESSE M. $(Q-12)$

PORTÅBLE GRAPHICS SYSTEM.

TIC,

1978́. 17P. MN (LA-UR-78-384)

KELLNER, RICHARD G, $(8-6)$

REED, THEODORE N. (C-6)

SOLEM, ANN (C-6)

\section{1-92}

PRELIMINARY CODE DEVELOPMENT FOR SEISMIC SIGNAL ANALYSIS RELATED TO TEST BAN TREATY QUESTIONS. 1977 BROLLEY, JOHN E., JR. (M-8)
$41-93$

PREPROCESSING OF PHERMEX FLASH RADIOGRAPHIC IMAGES.

LASL

1978; 18P. (LA-7105-MS) BROLLEY, JOHN E., JR. (N-8)

41-94

PREPROCESSING OF PHERMEX FLASH

RADIOGRAPHIC IMAGES WITH HAAR

AND ADAPTIVE FILTERING.

LASL,

1978, 6P. (LA-7561-MS) BROLLEEY, JOHN E., JR. (M-8)

41-95

PREPROCESSING OF SEISMIC SIGNALS FOK PATTERN RECOCNITION.

TIC, 1978 19P. MN (LA-UR-78-1507)
BROLLEX, JOHN E.., JR. (M-8)

41-96

PREPEOCESSING OF SEISMIC SIGNALS BY HAAR DECOMPOSITION AND ADAPTIVE FILTERING FOR PATTERN RECOGNITION. LASL 978: 15P (LA-7541 -MS) BROLLEY, JOHN E., JR. (M-8)

PROCEEDINGS OF THE 1978 LASL WORKSHOP ON VECTOR AND PARALLEL PROCESSORS, HELD AT LOS ALAMOS SCIENT IF IC

LABORATORY, LOS ALAMOS, NEW MEXICO, SEPTEMBER 20-22, 1978.

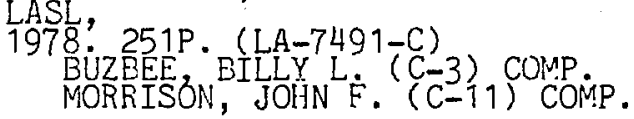

41-98

PRODUCT FORMULAS AND NICHOLSON

TYPE INTEGRALS FOR JACOBI

FUNCTIONS. I. SUMMARY OF RESULTS.

SIAM J. MATH. ANAIYSIS, V. 9 P P $76-86$. 1978.
DURAND, LOYAL (T-DO)

41-99

PROGRAM NICOLET TO INTEGRATE ENERGY LOSS IN SUPERCONDUCTING COILS. LASL, 6P. (LA-7428-MS VOGEL; HERBERT F. (CTR-9)

41-100

PROSPECTS FOR NUMERICAL SIMULATION

OF BLUFF BODY AERODYNAMICS

AERODYNAMIC DRAG MECHANISMS OF BLUFF BODIES AND ROAD VEHICLES, G. SOVRAN ET AL., EDS. P.313-55. PLENUM, 1978. HIRT CYRIL W. JR. $(T-3)$ 
41-101

QUANTITATIVE UNIVERSALITY FOR A

CLASS OF NONLINEAR TRANSFORMATIONS.

V. 19 TATIST: PHYS

FEIGENBAUM, MITCHELL J. (T-DOT)

41-102

REZONING - AN ADAPTIVE REFINEMENT

TECHNTOUE.

CUBE SYMPOSIUM. 1978. LOS ALAMOS,

NM. AEST. J.J. RUMINER, COMP.

P.51-2. LASL, 1978 . (LA-7417-C)
GUERRA, FRANCISCO M. $(W X-3)$

41-103

SCIENTIFIC CALCULATING SYSTEM

WITH A NUMBER - ORIENTED

MICROPROCESSOR SEQUENCED BY A

SINGLE CONPONENT MICROCOMPUTER.

TIC

1977. 5P. MN (LA-UR-77-2383)

ALSO PUBLISHED IN:

IEEE TRANS NUCL SCI

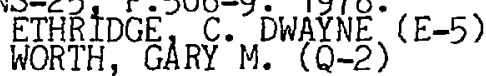

$41-104$

SECSIM - A COMPUTER SECURITY

SIMULATION CODE.

CUBE SYMPOSIUM. 1978. LOS ALAMOS,

NM. ABST. J.J. RUN INER COMP.

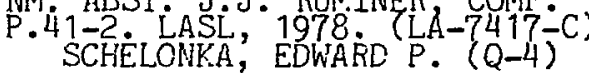

$41-105$

SECTIONED METHODS FOR

IMAGE RESTORATION

IEE TRANS. ACOUST. SPEECH,
SIGHAL PROCESSING, V.ASSP-26,

P. 157-64. 1978.

HUNT, BOB R.

41-106

SECTIONING IN IMAGE PROCESSING.

TIC, 797 PP. MN (LA-UR-77-259Q)

41-107

SIGNIFICANCE ARITHMETIC.

APPLICATION TO A PARTIAL

DIFFERENTIAL EQUATION.

IEEE TRANS. COMPUT.

V.C $-26, P .639-42.1977$.

ALSO PUBLISHED IN:

COMPUTER ARITHMETIC. 1975.

SYMPOSIUM. 3RD, SOUTHERH?

METHODIST UNIV'. DALLAS, TX.

PFOC P $64-6.1 \mathrm{IEE}, 1975$.

$13 / C 7380^{\circ} 1975$

BIVINS FOBERT L. (C-3)
METROPOLIS, NICHOLAS C. (T-7)
41-108

SIMULATION MODEL FOR BOOM TOWN HOUSING.

1973

PI $68 \mathrm{P}$. (LA-7324-MS)

FORD; ANDREW (S-2)

41-109

SOME CONDITIONS FOR WEAK

CONVERGENCE TO EQUILIBRIUM OF

NONLINEAP CONTRACTION SEMIGROUPS.

J. DIFFERENT. EQUA.

V. 29 PIMBLEY, $269-77$. 1978 .

NICHOLS; BASIL (T-7)

41-110

SOME L.INPACK TIMINGS ON THE

CRAY -1 .

VECTOR AND PARALLEL PROCESSORS.

1978. WORKSHOP LOS ALAMOS, NM

BUZBEE, COMP. PROC., P.58-75. LASL,

1978 DONGARPA, JACK J.

41-111

SOME TECHNIQUES FOR DIGITAL PROCESSING

DISPLAY AND INTERPRETATION OF RATIO

IMAGES IN MULTISPECTRAL REMOTE SENSING OPTICAL COMPUT ING CONFERENCE.

1977 INTERNATIONAL SAN DIEGO

IMÄGE PRỎCESSING. A.G. TESCHER,

ED. P. 47-54. SPIE, 1977 .

(621.3 A I610 1977 )

TIC,

1977 9P. MN (LA-UR-77-1877) WECKSUNG, GEORGE W. $(M-8)$
BREEDLOVE, JAMES R. $(M-8)$

41-112

SOME UNIQUE HEAT TRANSFER SOLUTIONS

IN WEAPON SYSTEM DESIGNS.

CUBE SYMPOSIUM. 1978 . LOS ALAMOS,

NM. ABST. J.J. RUMINER COMP.

JAEGER, 'DWIGHT L. (WX-3)

41-113

SPECTRAL ESTIMATION ACCURACY WITH AREITRARY TRUNCATING FUNCTIONS.

TIC, 11P. MN (LA-UR-77-2671)

LAHART, M. J. (M-8)

$41-114$

STANDARD FILE TRANSPORT PROTOCOL. LASI 1978: 25P. (LA-7388-MS) CHORN GRANVILLE E $(\mathrm{C}-4)$ CHRISTMAN, RONALD D. $(\mathrm{C}-4)$
KLINGNER, C. THOMAS $(\mathrm{C}-4)$ 
41-115

STUDY OF DATA COMMUNICATIONS AT THE LOS ALAMOS SCIENTIFIC LABORATORY, 1977 LASL,

1978: 22P. (LA-7361-MS)
LEWIS, RICHARD E. (C-DO-R)
GORE; RAYMOND A. (E-DO)

41-116 S2D - THE SESAME EQUATION OF STATE CUBE SYMPOSIUM. 1978. LOS ALAMOS, NM ABST. J.J. RUMINER COMP

P. 12. LASL, 1978 (LA-74 $47-\mathrm{C})$
JOHNSON, JAMES D. $\left(T^{4} 4\right)$

$41-117$

TERMS OF AN ARITHMETIC SEQUENCE

PRIME TO M.

$$
\text { 1978. 7P. (LA-7353-MS) }
$$

41-118

THERMAL ANALYS $\perp D$ OF A ROCKY FLATS EXPERTMENT.

CUBE SYMPOSIUM. 1978. LOS ALAMOS,

NM: ABST J.J RUIINER, COMP

vígIL, ARMÁNDO S. ( $W X-3)$

41-119

TIME - RESOLVED PHERMEX IMAGE

RESTORATIONS CONSTRAINED WITH AN

ADDITONAL MULTIPLY - EXPOSED IMAGE.

LASL

1978

KRUGER; RICHARD (N-8)

BREEDLOVE, JAMES R. $\left(M_{-}-8\right)$

$41-120$

TIME EVOLUTION OF ALMOST PERIODIC

SOLUTIONS OF THE KDV EQUATION.

ROCKY MT. J. MATH.

V.8,P. 95-104. 1078 .

41-121

TRANSFORMATIONS OF THE MULTIVARIATE

NORMAI DISTRIBUT IOH WITH

APPLICATIONS TO SIMULATION.

T1978. 4P. MN (LA-UR-77-2595) JOHNSON, MARK E. (Q-12) RAMBERG; JOHN

UNDERSTANDING MASS STORAGE.

MASS STORAGE WORKSHOP. '1977.

ED. P $15-34$ NCAR, 1978.

$\left(621.381 / N_{4} 14 S\right)$ WORLTON, WILLIAM J. (C-DO)
$41-123$

UNIFIED TIME DOMAIN ELECTROMAGNETIC

AND CIRCUIT ANAIYSIS CODE

CUBE SYMPOSIUM. 1978. LOS ALAMOS,

NM. ABST. J.J. RUMINER COMP

P. 57 LASL 1978 (LA $1417-C)$
LANDT, JEREMY A. (E-DOR)

UNIFi 24 MI.Y REDUNDANT ARRAYS.

T1977. 15P. MN (LA-UF-77-2683)

FENIMORE, EDWARD $(P-4)$
CANINON, THOMAS M. (M-8)

$41-125$

USER'S MANUAL FOR SIN - A ONE-

DIMENSIONAL HYDRODYMAM IC CODE FOR

PROBLEMS THAT JHCLUDE CHEMICAL

REACTIONS, ELASTIC - PLASTIC FLOW

SPALLING, PIASE TRANSITONS, MELTING,

FOREST FIRE, DETONATION! BUILD-UP, AID

SESAME TABULAF EQUATION OF STATE.

1978

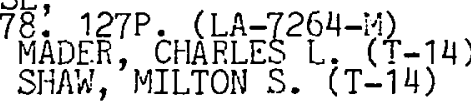

41-126

USING A MICKOCONPUTEP SYSTEM TO

CONTROL DATA COLLECTION IMSTRUMENTATION

OF A LASER DAMAGE EXPEFTMENT

CUBE SYPVOSIUM. 1978 . LOS ALAMOS,

NM. ABST. J.J. RUMINER CONP.

P.60-1. LASL, 1978 . (LA-7417-C)

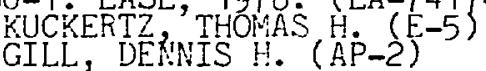

$11-127$

USING SYSTEM 2000 WITH PROPOSED AHSI STANDARDS FOR DATA EXCHANGE. TIC

1077 . 27P. M: (LA-UR-77-2'739)

BENKOVITZ CARMEN M.
WIIEY, RICHARD A. $(\dot{C}-3)$

$41-128$

VARIATIONAL DERIVATION OF A

CONTRIBUTION MONTE CARLO TECHIHOUE.

TRANS. AN . NUCL. SOC.

30 $P .587-8$. 1978

GERSTL S. A. IT $(T-1)$

DUEJ ARJE $(\bar{T}-1)$

POR'RANING, GERALD C.

41-129

VECTORIZATION FRON A LAEEE CONE

POINT OF VIEY

VECTOF AND PAEALIEL PSCCESSCFS.

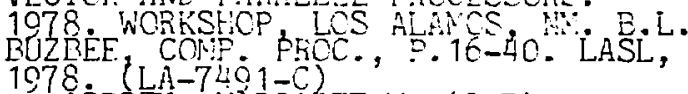
ASFEEY, $X$ KGASET $\because .(6-7)$ 
41-130

VECTORIZATION OF BLOCK RELAXATION

TECLINIQUES - SOME NUMERICAL

VECTOR AND PARALLEL PROCESSORS.

1078. WORKSHOP, LOS ALAMOS, NM. B.L.

BUZBEE, COMP PROC., P. 166-73. LASL,

1978. (LA-7491-C)

41-131

VECTORIZED PIC SIMULATION CODES

ON THE CRAY - 1 .

VECTOR AND PARALLEL PROCESSORS.

1978. WORKSHOP LOS ALAMOS, NM. B.L.

SUZBEE, COMP. PROC., P.41. LASL,

1978. (LA-7491-C)

FÖRSLUND, DAVID W. $(\mathrm{L}-6)$

41-132

VISUAL AIDS BY COMPUTER GRAPHICS.

CUBE SYMPOSIUM. 1978 . LOS ALAMOS,

NM ABST, J J RUMINER, COMP.

EWALD, ROBERT H. (C-6) 
43. NUCLEAR PROPULSION SYSTEMS AND AERCSPACE SAFETY

43-1

NUCLEAR REACTORS FOR SPACE ELFCTRIC

WER

1978: 36P. (LA-7290-SR)

BUDEN, DAVID (Q-DO)

ST3-2

REACTORS FOR SPACE ELECTRIC POWER. TIC

1978. 14P. MN (LA-UR-78-1306)

BUDEN, DAVID (Q-DO) 


\section{4-1}

GEOSTATISTICS PROJECT OF THE

NATIONAL URANIUM RESOURCE EVALUATION

PROGRAM, JANUÁRY - MARCH 1978.

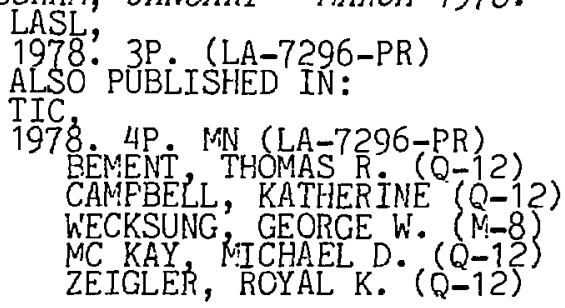

$44-2$

GEOSTATISTICS PROJECT OF THE

NATIONAL URANIUM RESOUPCE

EVALUATION PROGRAM, APRIL - JUNE 1978 .

LÁSL

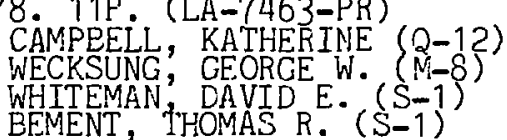

44-3

CEOSTATISTICS FROJECT OF THE NATIONAL URANIUM RESOURCE EVALUATION PFOGRAM, OCTOBER-DECEMBER 1977.

LASL

1978. 4P. (LA-7168-PR)

BEMENT, THOMAS R. $(Q-12)$
MC KAY' MICHAEL D $(q-12)$
WECKSUNG, GEORGE $\cdot(q-8)$

44-4

NDA TECHNOLOGY FOR URANIUM RESOURCE

EVALUATION, JULY 1-SEPTEMBER 30, 1977. LASL,

1978' 23P . (LA-7139-PR)

EVANS, MICEIAEL L. $(Q-1)$ COMP.

N4-5 TECHNOLOGY FOR URANIUNY RESOUPCE

EVALUATION OCTOBER 1-DECEMBER 31,

1977

LASL

1978: 10P. (LA-7251-PR)

MENLOVE, HOWARD O. (Q-1) COMP.

44-6

HON-DESTRUCTIVE FIELD MEASUREMENT

OF THE RATIO URANIUM -235\%

URANIUM $-23 E$ IN DEPLETED TO

MODERATELY ENRICHED URANIUM.

TIC, 10P. MN (LA-UR-77-2850)

BÁLAGNA, JOHN P: JR: (CNC-11)
COWAN, GEORGE A:'(CHC-DO) 
45-1

CHARACTERISTICS OF WASTES AND

SOILS WHICH AFEECT TRANSPORT OF

RADIONUCLIDES THROUGH THE SOIL AND

THEIR RELATIONSHIP TO WASTE MANAGEMENT JULY 1, $197 \overline{6}$ - SEPTEMBER 30, 1977.

LASL,

FOWLER, (LA-7311-PR)

PSSINGTON, EDWARD H. $(\mathrm{H}-12)$

DISP-20ิSAL OF HAZARDOUS WASTES.

ENVIRON. SCI. TECHNOL.,

BARNHART, BENJAMIN J. (H-9)

$45-3$

EXPERTMENTAL STUDIES OF THE

DEGRADATION OF RAD-WASTES FOR THE

SANDIA LABORATORIES WASTE ISOLATION

PILOT PROJECT (WIPP), OCTOBER 1 -

DECEMBER 31, 1977 .

$$
\begin{aligned}
& \text { LASL, } 5 \text { P (LA-7246-PR) } \\
& \text { ZERWEKH, AL (CMB-1) } \\
& \text { KOSIEWICZ, STANLEY (CMB-1) }
\end{aligned}
$$

45-4

EXPERIMENTAL STUDIES OF THE

DEGRADATION OF RAD-WASTES FOR THE

SANDIA LABORATORIES WASTE ISOLATION

PILOT PROJECT (WIPP), JANUARY 1 -

MARCH 31, 1978.

$$
\begin{aligned}
& \text { 1978: 9P (LA-7259-PR) } \\
& \text { ZERTEKH, AL CMB-1) } \\
& \text { KOSIEWICZ, STANLEY (CMB-1) }
\end{aligned}
$$

\section{5-5}

EXPERIMENTAL STUDIES OF THE

DEGRADATION OF RAD-WASTES FCR THE

SANDIA LABORATORIES WASTE ISOLATION

PILOT PROJECT (WIPP), APRIL 1 -

JUNE $30,1978$.

LASL,

1978: 5P. (LA-7478-PR)

ZERWEKH, AL (CMB-1)

$\left.\begin{array}{l}\text { KOSIEWICZ, STANLEY } \\ \text { BARRACLOUGH, BRUCE } \\ \text { CME-1 } \\ \text { CMB-1 }\end{array}\right\}$

45-6

GEOLOGIC DESCRIPTION OF CORES

FROM HOLES P-3 MHI- 1 THROUGH P-3 MH-5, AREA G, TECHNICAL AREA 54.

LASL, 13P. (LA-7308-MS)

PURTYMUN, WILLIAM D. $(\mathrm{H}-8)$

WHEELER, MERLIN L. (H-8)

ROGERS, MARGARET ANNE (H-8)
45-7

LASL MODELS FOR ENVIRONMENTAL TRANSPORT OF RADIONUCLIDES IN FORESTS.

$$
\begin{aligned}
& \text { TIC } \\
& \text { 1978. 9P. MN (LA-UR-78-1097) } \\
& \text { GALIEGOS, ANTHONY F ( } 1-12) \\
& \text { SMITH, W. JOHN, II (H-12) } \\
& \text { JOHNSON, LAMAR J. (H-DO) }
\end{aligned}
$$

45-8

LOW - LEVEL SOLID RADIOACTIVE WASTE DISPOSAL.

LASL

MINI-REVIEW. 1978. 4P.

(LASL-78-5) JOHN LAWRENCE (H-7)

MOVEM-9ENT OF FLUIDS AND PLUTONIUM

FROM SHAFTS AT LOS ALAMOS, NEW MEXICO.

1978: 6P. (LA-7379-MS)

PUPTYMUN WILLIAM D; $(\mathrm{H}-8)$

GARDE, RAYMOND $(\mathrm{H}-1)$

PETERS, RICHARD $\mathrm{J}$. $(\mathrm{H}-8)$

45-10

NUCLEAR WASTE MANAGEMENT TECHNOLOGY DEVELOPMENT PROCRAM, JANUARY DECEMBER 1977.

LASL

1978. 29P (LA-07501-PR) JOHNSON, LAMAR J- (H-DO) COMP.

45-11

PROCESSING AND DISPOSAL OF

FADIOACTIVE WASTE BY THE WASTE

MANAGEMEITT GROUP AT LASL.

LASL

MINI-REVIEW. 1978. 4P. (LASL-78-83) KEENAN, THOMAS K. $(H-7)$

45-12

STRATIGRAPHY OF THE BANDELIER TUFF IN THE PAJARITO PLATEAU.

APPLICATIONS TO WASTE MANAGEMENT.

LASL,' 57P. (LA-7225-MS)

CROWE, BRUCE M. $\left(\begin{array}{c}G-5 \\ G-6\end{array}\right.$

LINN, GEORGE W, $(G-6)$

BEVIER, MARY LOU 
45-13

WASTE MANAGEMENT CAPABILITIES FOR ALPHA

BEARING WASTES AT THE LOS ALAMOS

SCIENTIFIC LABORATORY.

TTC
1977 . 12P. MN (LA-UR-77-2686)
KEENAN; THOMAS K. $(\mathrm{H}-7)$

WASTE MANAGEMENT PROGRAMS AND

CAPABILTTIES WITHIN THE LOS ALAMOS

SCIENTIF IC LABORATORY.

LASL

1978: 57P. (LA-7548-MS)

ERDAL BRUCE R. (CNC-11)

HOFFMAN, DARLEANE C. (CNC-11)

LUTH, W. C.

GRAVES, GLEN A. (DIR OFF)

JENSEN, REED J.ं (AP-DO)

ZERWEKH, AL (CME-1)

ERDAL GRUCE R. (CNC-11)

GERMAIN, LAWREMCE S. (J-DOT)

JOHNSON, LAMAR J. (H-DO)

KILLIAN; BARBARA GERMAIN (J-9)

WALTON, ROCDY B. $(Q-1)$

MREIWALD JOYCE:G: $(\mathrm{H}-8)(\mathrm{T}-1)$ 
46-1

CHARACTERIZATION OF DEVONIAN SHALES

USING LASER PYROLYSIS - GAS

CHROMATOGRAPHY.

TIC7 11P. MN (LA-UR-77-2106)

VANDERBORGH, NICHOLAS E, (CMB-8)

WILLIAMS, JOZEL M. (CMB-8)

WEWERKA, EUGENE M. (CMB-8)

BERTINO; JAMES P. ( CME-8)

$46-2$

DATA - B'SE MANAGEMENT SYSTEM FOR

SPATIAL DISPLAY OF FEDERAL OFFSHORE OIL ANL GAS LEASE DATA.

TIC,

PHILLIPS, RICHARD

LOHRENZ, JOHN

$46-3$

EFFEETS OF VARIOUS PYROLYSIS

PRODUCTS FROM GREEN RIVER CIL

SHALE.

1978: 99P. THESIS, UNM,

ALBUQUERQUE, NM, 1977.

FRITZ, GEORGIA T. (WX-2)

46-4

EXPLOSIVELY PRODUCED FRACTURE

OF OIL SHALE, JANUARY - MAFICH 1978.

LASL

1978: 14P. (LA-7338-PR)

CARTER, WILLIAM J. (G-7) COMP.

$46-5$

EXPLOSIVELY PRODUCED FRACTURE

OF OIL SHALE, APRIL - JUNE 1978.

LASL,

1978: 26P. (LA-7438-PR)

CARTER, WILLIAM J. (G-7) COMP.

46-6

EXPLOSIVELY PRODUCED FRACTURE OF OIL SHALE, OCTOBER - DECEMBER 1977.

1978: 45P. (LA-7164-PR)
CARTER, WILLIAM J. (G-7) COMP.

46-7

EXPLOSIVELY PRODUCED FRACTURE OF OIL SHALE APRIL - JUNE 1977.

1978: 47P. (LA-6901-PR)

CARTER, WILLIAM J. (G-7) COMP.
EXP-E SIVELY PRODUCED FRACTURE OF OIL ¿ULY - SEPTEMBER 1977.

1978 CARIER, WILLIAM J. (G-7) COMP.

46-9 SHALE APRIL, 1977 - MARCH, 1978. 1978; 108P. (LA-7357-PR) CARTER, WILLIAM J. (G-7) COMP.

46-10

MAPPING OFFSHORE OIL LEASES. TIC. 1978 SP. MN (LA-UR-77-2892)

46-11

PERMEABIJITY ENHANCEMENT USING

EXPLOSIVE TECHNIOUES EHERGY CONVERSION ENGINEERING

CONFERENCE. 1978 (AUG.) 13TH

INTERSOCIETY, SAN DIECO, CA. PROC., ÁLSO PÜBLISHED IN!

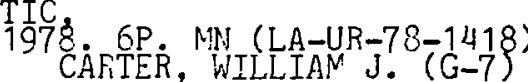


47-1

ANNOTATED BIBLIOGRAPHY OF LAMPF

RESEARCH AND DEVELOPMENT.

LASL, 261P. (LA-7431-MS

JAMESON, ROBERT A (MP-9)

ROYBAL, ELIZA U. (MP-9)

47-2

ANYOTATED BIBLIOGRAPHY ON

HIGH - INTENSITY LINEAR

ACCELERATORS.

1978: 290P. (LA-7124-MS)

JAMESON, ROBERT A. (MP-9)

ROYBAL, ELIZA U. ( $M P-9)$

BEAM PROF ILE MONITOR FOR SECONDARY BEAMS.

WUCL. INSTRUM. METHODS,

V. 156 P $365-7.1978$.
KRAUSSE GEORGE J. I III (MP-4)
GRAM, PETER A. M. (MP-4)

$47-4$

BEAM TOMOGRAPHY OR ART IN

ACCELERATCR PHYSICS.

LASL

1978. 21P. (LA-7498-MS)

FRASER, JOHN S.

$47-5$

BEAM VOLTACE AND CURRENT

PARAMETER STUDY FOR THE

AUTORESOH:ANT ACCELERATION

PROOF-OF-PRIHICIPLE EXPER IYENT.

1978. 8P. (LA-7568-MS)

GODFREY BRENDAN $\mathrm{E}$; $(\mathrm{T}-15)$

47-6

COLLECTIVE IOIN ACCELERATION

THROUGH TEMPORAL MODULATION OF

RELATIVISTIC ELECTRON BEAM ENERGY.

PHYS. REV. LETT.

V. 40 P. 1137-40. 1978

FADHLEY, ERENDAN $\mathrm{B}$. (T-15)

47-7

COMPACT $250 \mathrm{KV}$ INJECTOR SYSTEM FOR

PIGNI

BULLL. AN ${ }^{\text {PHYS }}$ SOC.,

HAMM, ROBERT W. (MP-12)

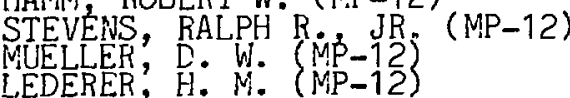

47-8 8 STUDY FOR ACHROMATIC AND ISOCHRONOLS 180 DEGREE BENDING SYSTEMS FCR PIGMI.

$$
\begin{aligned}
& \text { LASL, } 20 P \text {. (LA-7406-IMS) } \\
& \text { INOUE,H. DONALD A. (AT-1) } \\
& \text { SWENSON, DONALD }
\end{aligned}
$$

47-9

DEVELOPMEIT OF SAMARIUM - COBALT

QUADRUPOLE LENSES FOR PARTICLE

ACCELERATOR APPLICATIONS.

LASL,

1978 14P. (LA-7232-MS)

BUSH, EDGAR D. JR. $($ MP-8)
SWENSON, DONALD A. (AT-1)

$47-10$

EXPERIENCE WITH THE LAMPF MINERAL INSULATED MAGNETS.

TIC, 7P. MN (LA-UR-77-1789)

ALSO PUBLISHED III:

MAGNET TECHNOLOGY.

1977 (AUG.29-SEPT 2). INTERNATIONAL

CONFERENCE 6TH, BRATISLAVA

CZECHOSLOVAKIA. 'PROC, P.551-6.

ALFA BRATISLAVA 1978 ' HARVEY, ALEXANDER (MP-13)

47-11

EXPERIMENTAL PROGRAM TO VALIDATE

ANALYSES OF ACCELERATOR BREEDER CONCEPTS.

TIC,

1977 . 32P. IN (LA-UR-77-2552)

TALBERT, $N$. L. JR. $(Q-14)$

RUSSELL; GARY $\}$ (P-11)

MALENFANT, RICHÄRD E. (Q-DO)

$47-12$

LAMPF (1975-1976) RESEARCH

HIGHLIGHTS AT THE CLINTON P.

ANDERSON MESON PHYSICS FACILITY.

LASL

1978. 34P. (LA-7201-MS)

BOWMAN, J. DAVID (MP-4) COMP.

$47-13$

LAMPF 201 . 25-MHZ LINAC FIELD

DISTRIBUTION.

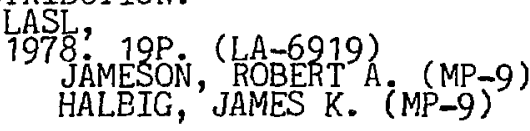


47-14

LONGITIDINAL TUNING OF THE LAMPE

201 . 25-MHZ LINAC WITHOUT SPACE CHARGE.

1978

78. 65P. (LA-6863)

JAMESON, ROBERT A. (M:P-9)

JULE, WTLLARD

MILLS, RENE SCOTT (MP-9)

BUSH, EDGA D. JR. (MP-8)

GLUCKSTERN, ROBEERT L. (MP-8)

47-15

MAGNETIC REFRIGERATOR DEVELOPMENT
DECEMBER
1,1977 - FEBRUARY 28, 1978.

1978: 11P. (LA-7257-PR)

BARCLAY, JOHN A. $(Q-10)$ COMP.
STEYERT, WILLIAM A., JR. $(Q-10)$ COMP.

$47-16$

MECHANICAL DESCRIPTION OF PIGMI.

BULL. AM PHYS SOC.,

HANSBOROUGH; LASH D. (AT-1)

BUSH, EDGAR'D. JR. (MP-8)

HART, VALGENE E. (MP-8)

47-17

MEDIUM - ENERGY PHYSICS PROGRAM,

AUGUST 1-OCTOBER 31, 1977.

1978: 81P. (LA-7268-PR)

VAN DYC , OLIN B. (MP-13) COMP.
DUNN, ELEANOR D. (MP-DO) COMP.

MEDIUM - ENERGY PHYSICS PROGRAM, MAY 1JULY $31,1977$.

LASL,

VAN DYCK, OLIN B. (MP-13) COMP.

DUNN, ELEANOR D. (MP-DO) COMP.

47-19

MEDIUM-ENERGY PHYSICS PROGRAM,

NOVEMBER 1, 1977 - JANUARY 31, 1978.

LASL

1978: 80P. (LA-7457-FR)

VAN DYCK, OLIN B. (MP-13) COMP.

DUNN, ELEANOR D. (MP-DO) COMP.

$47-20$

MICROPHYSICS OF CLOUDS AND

PRECIPITATION

REIDEL, 1978

(551.5\%PQ72M)

PRUPPACHER, HANS R.

KLETT, JANES D.

MUON SPECTRA FROM PION PRODUCTION

TARGETS.

LASL, $30 \mathrm{P}$ (LA-7222-MS)
TSCHALAR, C.
$47-22$

NEW DEVELOPMENTS IN ACCELERATCR

TECHNOLOGY AT LASL.

BULL. AM P PHYS SOC.

STOVALL, JAMES $\dot{E} .($ AT-1)

OPTIMIZATION OF THE DISK AHD WASHER ACCELERATING CAVITIES.

LASL

1978. 14P. (LA-7407)

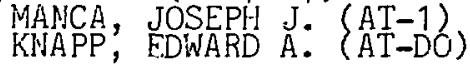

OVERVIEW OF LAMPF AND ITS ACCOMPLISHMENTS.

LASL,
1978 ROSEN, LOUAS (NP--DO) 47-25
POSTTION - SENSITIVE GAS PROPORTIONAL
CHAMBERS.

${ }_{197}^{T}$ T 4P MN (LA-UR-77-2438)
MORRIS CHR ISTOPHER L $(M P-10)$
THIESSEN, HIERY A $(M P-10)$

$47-26$

SHEAP 77. SYMPOSIUM OF MORTH-EASTERN ACCELERATOR PEFSOHNEL HELL AT THE

LOS ALANOS SCIENTIFIC LABCRATORY,

LOS ALAMOS NEN MEXICO,

LASL

1978 WOODS, RICHARD $(P-9)$ COMP.

47-27

SOME NEW ACCELERATIHG STRUCTURES FOR HIGH CURRENT INTENSITY ACCELERATORS.

1978. \&P. (LA-7157-MS)

MANCA, JOSEPH J. (P-5)

$47-28$

TMO1 MODE ACCELERATING CAVITY

OPTIMIZATION.

LASL

1978. 23P. (LA-7323)

MANCA, JOSEPH J. $\left(\begin{array}{l}A T-1 \\ \text { KINAPP, EDWARD }\end{array}\right.$

47-29

TRANSIENT THERMAL STRESS ANALYSIS

OF A PROPOSED PION PRCDLICTION

TARGET.

1978 .

LINDQUIST, LLOYD O. (MP-7)
SCARBROUGH, EDWAFD C. (MP-7) 


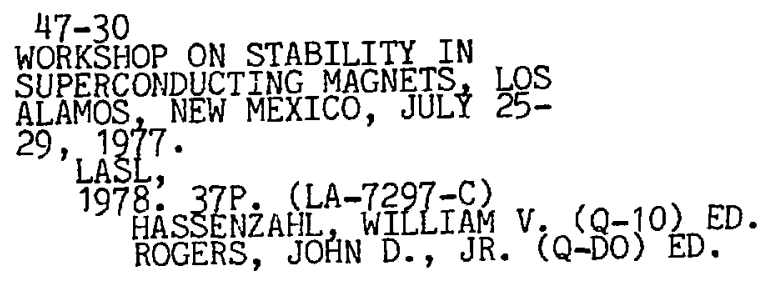


48-1

COLOR MOVIE SIMULATION OF A GAS - WATER RESERVOIR.

TIC78́. 9P. MN (LA-UR-77-2447)

ALSO PUBLISHED IN:

SIMULATION SYMPOSIUM.

1978. 11TH ANNUAL TAMPA, FL-102.

THE SYMPOSTUM 1978 .

JOHNSON, MARK E. (Q-12)

MONASH,'ELLIS A.

48-2

EVALUATION OF METHODS FOR

STIMULATION AND CHARACTERIZATION

OF EASTERN GAS SrALES, APRIL-

JUNE 1977 .

LASL,

1978.: 23P. (LA-7094-PR)

CARTER, WTLLIAM J. (G-7) COMP.

VANDERBORGH, NICHOLAS E. (G-7) COMP.

GAS-3 STIMULATION STUDIES AT LASL.

TIC $77^{2}$. 27P. MN (LA-UR-77-1937)

CARTER; WILLIAM J.' $\left(\mathrm{N}_{\mathrm{j}}-6\right)$

OLINGER, BARTON W: $(M-6)$

VANDERBORGH, NICHOLLAS E $($ CME-8)

48-4

HYBRID COMPUTERS FOR SIMULATION OF GAS DIFFUSION IN FRACTURED SHALE.

TIC,

11P.MN (LA-UR-77-2066)

48-5

INVESTIGATION OF THE LOGNORMALITY

OF OFFSHORE OIL AND GAS LEASE

BIDDING DATA. CRAMER-VON MISES

ONE - SAMPLE TEST.

LASL

1978: 27P. (LA-7339-MS)

GENTER, F. C.ं. LANENCE A. (S-1)

MER-6 (MAXIMUM FFFICIENT RATE) USING A

GAS RESERVOIR MODEL WITH WATERFLOODING.

\section{LASL}

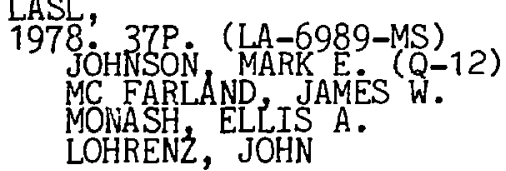

48-7

MODELING AND OPTIMIZING A GAS WATER RESERVOIR.

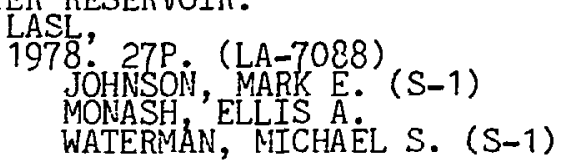

LASL,

1978: 27P. (LA-7088)

JOHNSON, MARK E. (S-1)

MONASH, ELLIS A.

WATERMAN, MICHAELL $S .(S-1)$

48-8

ON THE PFOBABILITY DISTRIBUTTON OF BIDS ON OUTER CONTINENTAL SHELF OIL AND GAS LEASES.

LASL, 20P. (LA-7190-MS)

$$
\text { BOHCKSER, MAWRENCE A. }(Q-12)
$$

48-9

SIMULATION STUDY OF MER FOR GAS - WATER RESERVOIRS.

LASL,

1978. 34P. (LA-6866-MS)

MP FARLAND JAMES W. $(E-4)$

MONASH, ELLIS A.

LOHRENZ, JOHN

$48-10$

STIMULATION AND CHARACTERIZATION

OF EASTERN GAS SHALES,

OCTOEER-DECEMEER 1977?

LASL, 18P. (LA-7383-PR)

SCHOTT; GARRY

VANDERBORGH, NICHOLAS E. (G-7)

48-11

STIMULATION AND CHARACTERIZATION OF

EASTERN GAS SHALES, JULY -

SEPTEMBER, 1977.

LASL,

1978 'ं 4OP. (LA-7320-PR)

SCHOTT, GARRY L. (G-7) COMP.

CARTER' WILLIAM J J (G-7)
VANDERBORGH, NICHOLAS E. (G-7)

48-12

STIMULATION AND CHARACTERIZATION OF EASTERN GAS SHALES,

$$
\begin{aligned}
& \text { LASL, } \\
& \text { 1978, OP (LA-7564-PR) } \\
& \text { SCHOOTT, GARRY L- }(\mathrm{G}-7) \text { COMP. } \\
& \text { VAMDERBORGH, NICHOLAAS E. (G-7) }
\end{aligned}
$$


$50-1$

AB INITIO CALCULATION GF THE

VIERATIOHAL EIVERGY TRAISFER RATE

OF IYYDROGEN IN ARGON USINC YONTE

CARLO CLASSICAL TRAJECTORIES AND

THE FORCED QUANTUM OSCILLATOR

MOLEL.

J. 'CHEM. PHYS.' 1978.

TLAIS WOEMAND C $(\mathrm{CNC}-2)$

$5 \mathrm{C}-2$

$A B$. INITIO EFFECTIVE CORE

POTENT IALS FCR MOLECULAR

CALCULATIONS. II ALL AND

MODIFICATIONS OF THE PROCEDURE.

$$
\begin{aligned}
& \text { J. CHEM. PHYS } \\
& \text { V. } 68 \text { P } 3059-66.1978 . \\
& \text { HAY P P JEFFREY (T-12) } \\
& \text { WADT, WILLARD R. (T-12) } \\
& \text { KAHIN, LUIS R. }
\end{aligned}
$$

$50-3$

AB IHITIO STUDJES OF GOLD

HYDRIDE, GOLD CHILORIDE, MERCURY

HYDRIDE AND MERCURY CHLORIDE

USING RELATIVISTIC EFFECTIVE

CORE PCTENTIALS.

J. CHEM. PHYS: 1978 .

WADT, WILLARD R. (T-i2)

KAHIN, LUIS $R$.

BOBRÓWICZ, FRANK W.

$50-4$

AB INITIO STUDIES OF THE METAL - METAL

BOND IN DIRHEHIUN: OCTACHLORIDE.

J. AM. CHEM SOC. 100, P. $2897-8.978$.

HAY, $P$. JEFFमEY $(T-12)$

$50-5$

ABSOLUTE INTEGRATED INTENSITIES

OF THE INFRARED ABSORPTION BANDS

OF TITANIUM TETRACHLORIDE.

J. CHEM. PHYS. ,

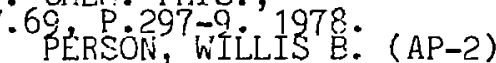

MAIER, WILLIAM B., II (AP-4)

$50-6$

ABSOLUTE OSCILLATOR STRENGTHS FOR

NEUT RAL ATOMIC URANIUM.

$$
\begin{aligned}
& \text { J. OPT SOC. AM. } \\
& \text { V.68 P. } 1173-81 \text { ' } 1978 . \\
& \text { BIENIEWSKI, THOMAS M. (CME-1) }
\end{aligned}
$$

AC CONDUCTIVITY OF A ONE-

DIMENSIONAL SITE - DISORDERED

LATTICE.

$$
\begin{aligned}
& \text { BULL: AM PHYS, SOC., } \\
& \text { ALBERS ROBERT C. (T-11) }
\end{aligned}
$$

$50-8$

AC CONDUCTIVITY OF A ONE-

DIMENSIONAL SITE - DISORDERED

LATTICE.

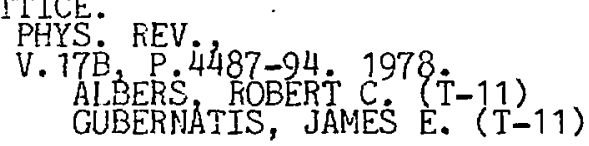

$50-9$

ACCURATE HYDROGEN (SUB 3) DYNAMICS ON AN ACCURATE HYDROGEN (SUB 3) POTENTIAL SUPFACE.

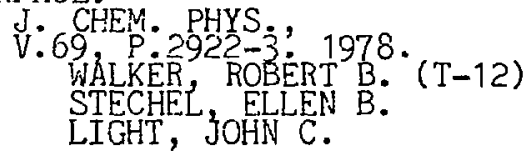

$50-10$

ANALYSIS OF 3 NU/SUB 3/ IN

SULFUR HEXAFLUORIDE.

J. CHEM. PHYYS: 1978.

GALBRA ITH, HAROLD W. $(T-12)$
ACKERHALT, JAY R. $\left(T-1 \frac{T}{2}\right)$

$50-11$

ANISOTROPIC POTENTIALS AND THE

DA NPING OF RAINBOW AND DIFFRACTION

CSCILLATIONS IY DIFFERENTIAL CROSS

SECTIONS.

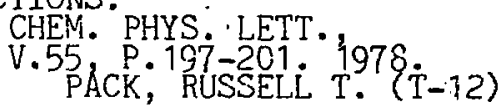

$50-12$

CALCULATIONS OF THE ELECTRONIC STRUCTUR AND TRAISITIONS OF ACTINIDE METAL HEXAFLUORIDES

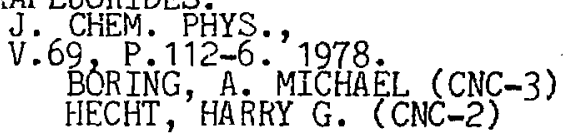


$50-13$

CALCULATIONS OF THE TEMPERATURE DEPENDENCE OF THE MULTIPHOTON ABSORPTION SPECTRUM OF SULFUR HEXAFLUORIDE.

OPT. LETT.

V. 3ं P $10 \dot{9}-11.1978$. ACKERHALT, JAY R. (T-12)

$50-14$

CARBON -13 RELAXATION TIMES IN A

HYDROCARBON CHAIN UNDERGOING

GAUCH-TRANS ISOMERISM.

BIOMOLECULAR STRUCTURE AND

FUNCTION. 1977 (MAY) SYMP.

UNIV. OF MISSOURT, COLLUNEIA' PROC.

P.F. AGRIS, ED ${ }_{19}$ ' $79-35$. ACADEMIC, LONDON ROBERT E. ( CNC-4)

AVITABILE, JOHN W., JR. (T-10)

COL-15

INFRARED PHOTON ABSORPTION

IN SULFUR HEXAFLUORIDE.

OPT. LETT
V. 3 Q $106-8.1978$,
QUIGLY', G. P. (AP-3)

$50-16$

COLLISIONLESS MULTIPLE PHOTON

EXCITATION OF SUFUR HEXAFLUORIDE. A COMPARISON OF ANHARMONIC

OSCILLATORS WITH AND WITHOUT

OCTAHEDRAL SPLITTING IN THE

PRESENCE OF ROTATIONAL EFFECTS.

J. CHEM. PHYS.,

V. 69 P. $1200-6: 1978$.

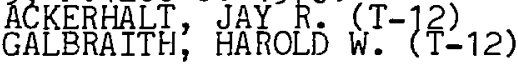

COMMENT ON "THEORY OF RESONANCE

FLUORESCENCE"

PHYS 17 AEV P. $471-2.1978$.

ACKERHALT, JAY R. (T-12)

$50-18$

COMPARISON OF MULTIPLE - PHOTON

EXCITATION MODELS.

OPT. LETT.
V. 3 P $15 \dot{2}-3 ; 1978$.
GALBRAITH, HAROLD W. $(T-12)$
ACKERHALT, JAY R. (T-12)

$50-19$

CONVERGENCE STUDIES OF LOW ENERGY STATIC E-NITROGEN COLLISIONS.

$$
\begin{aligned}
& \text { J. PHYS. B, } \\
& \text { V. 10 P L 19-23. } 1977 \text {. (T-12) } \\
& \text { COLRISON, MICHAEL A. LEE A. }
\end{aligned}
$$

$50-20$

CORRELATIONS AND SELF-COHSISTENCY

IN PION SCATTERING. (I)

NUCL V. PHYS

JOHNSON, MIKKEL (MP-DO)

BETHE, HANS A.

$50-21$

COVALENT AND IONTC STATES OF

THE RARE GAS MONOFLUORIDES.

$$
\begin{aligned}
& \text { J. CHEM. PHYS. } \\
& \text { V.69 P P } 134-49.1978 . \\
& \text { DUNNING, TEOMAS H. JR. (CNC-2) } \\
& \text { HAY, P. JEFFREY (T'-12) }
\end{aligned}
$$

$50-22$

COVALENT AND IONIC STATES OF THE XENON HALIDES.

$$
\begin{aligned}
& \text { J. CHEN. PHYS. } \\
& \text {-69 PAY } 2209-20 \text {. } 1978 . \\
& \text { DUNAIING, THOMAS H., JK. }
\end{aligned}
$$

$50-23$

DETAILED QUANTUM TRANSITION STATE THEORY APPLICATION TO COLLINEAR REACTIONS.

TIC7 8 . 48P. MN (LA-UR-78-649)

50-24
DIATOMIC - MOLECULE VIBRATIONAL

POTENTIALS - ACCURACY OF

REPRESENTATIONS

J. CIIEM. PHYS.

V. 68 ERGELKE, RAY $\left(M_{1}-3\right)$

50-25

DIFFUSION TENSOR FOR ATCHIC

MIGRATION IN CRYSTALS.

P. $18 \dot{8}$. KE $20350-9,1978$ LARSEN, ECWALD W. $(\mathrm{T}-1)$

$50-26$

DIHEDRAL ANGLE DEPENDENCE OF

GEMINAL, SCALAR COUPLING CONSTANTS

II CARBON: $-13(1)$ AMINO SUGARS.

CARBOHYDR. RES

V.60 P.9-18. 1978. WALKER, THOMAS E. $(\mathrm{CNC}-4)$

BARKER ROBERT

MATWIYÓFF, NICHOLAS A. (CNC-4)

50-27

DIODE LASER MEASUREMEITS OF AMMONIA

ABSORPTION LINES AROUND 10.6

MICROMETERS

J. MOL. SPECTROSC

V. 69 PER $489-93$ S $1 \dot{\mathrm{G}} 78 .(\mathrm{AP}-4)$ 
$50-28$

DIPOLE INTERACTIONS AND TRAPPING EFFECTS

OF POSITIVE MUDNS IN FCC AND ECC METALS. J. NUCL, MATER
V.69 \& 70, P. $13-5.1978$.
STRAUE, GALEN K (T-4)
PARKIN, DON M. (CMB-8
LYNN, K. G.

50-29

DISSOCIATION OF NITROGEN TRIFLUORIDE

IN SHOCK WAVES.

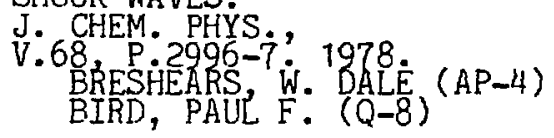

$50-30$

DISSOCIATIVE ATTACHMENT GF ELECTRONS

TO FLUORINE

APPL. PHY S. LETT
V. 33 P $56 \dot{9}-71$ Tó
SCHNEIDER BARR $(T-12)$
BRAU, CHARLES (AP-2)

50-31

DYNAMIC ELECTRON SCREENING IN NUCLEAR AMD MESONIC ATOM E 1 TRANSITIONS.

NUCL. PHYS

V.A2 $98, P .333-43.1978$.

LEOH, RYLVIN (MP-3)

$50-32$

EFFECTS OF MOLECULAR ROTATION

AND VIBRATION ROTATION INTERACTION

ON! COLLISIONLESS MULTIPLE

PHIOTON EXCITATION OF SULFUR

HEXAFLUORIDE.

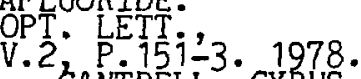

CANTRELL, CYRUS D, III $(\mathrm{T}-12)$

$50-33$

ELECTRON TRANSFER IN COLLISIONS

BETWEEN ATOMIC IONS AND RARE-GAS

ATOMS FOR PRIMARY-ION ENERGIES BELOW 200 EV. II.

J. CHEM: PHYS

V. 69 PAIER, WILLIAM $30 .$, II (L-3)

$50-34$

ELECTRON TRANSFER IN COLLISIONS OF DOUBLY CHARGED ATOMIC IONS WITH

RARER - GAS ATOMS FOR PRIMARY - ION

ENERGIES BELOW 100 EV.

BULL. AM PHYS. SOC.

V.23. P. 149-50. 1978 .'

II $(\mathrm{AP}-4)$
ELECTRON TRANSFER IN COLLISTONS OF DOUBLY CHARGED ATOMIC IONS WITH RARE GAS ATOMS FOR PRIMARY - ION ENERGIES BELOW 100 EV.

J. CHEM. PHYS

V.68, P: 4228-33. 1978 MAIER WILLIAM BA. II (AP-4)

$50-36$

ELFCTRONIC STATES OF ARCON FLUORIDE AND KRYPTON FLUORIDE.

J. CHEM. PHYS, 3 WADT, WILLARD R. ('T-12) HAY,'P. JEFFREY $(T-12)$

ELEC 37 TRONIC STATES OF CHROMIUM CARBONI J.: AM. CHEM SOC.' 1978 HAY,$\dot{P}^{-}$JEFFREY $(T-12)$

$50-38$

ELECIRONIC STRUCTURE AND RADIATIVE PROPERTIES OF THE RARE GAS HALIDES. VULL. AM. PHYS. SOC., HAY $P 2$.JEFFREY $(T-12)$ DUNHING, THOMAS H., JR. (CNC-2)

$50-39$

ELECTRONIC STRUCTURE AND SPECTRAL PROPERTIES OF RARE - GAS DIMER

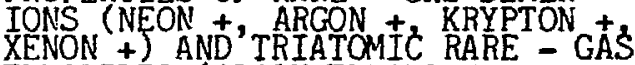
FLUORIDES (ARGON FLUORIDE, KRYPTON FLUORIDE)

BULL: AM PHYS: SOC.,

WADT, WILLARD CARTWIGHT DAVID C. (T-12)

COHEN, JAMES S. $\left\{\begin{array}{l}\mathrm{T}-12 \\ \mathrm{~T}-12\end{array}\right\}$

$50-40$

EIIERGY SPECTRUM OF STOPPING NEGATIVE MESONS AND THE CONCENTRATION DEPENDENC OF CAPTURE ERACTIONS.

PHYS. REV. $112-5 \cdot 1978$;
V. T7A, P. $112-5$.
LEON, MELVIN (MP-3)

$50-41$

EPR STUDIES OF VALENCE FLUCTUATING

CERIUM ( $1-X)$ THORIUM $(X)$.

V. $23, P .232,1978$

SUGAWARA, K.

SCHMIDT, $P$. $H$. 
$50-42$

ERRATUM. CARBON -13 NMR OF CARBON

MONOXIDE. I. PRESSURE. DEPENDENCE OF

TRANSLATIONAL MOTION IN BETA CO.

$$
\begin{aligned}
& \text { J. CHEM. PHYS. } \\
& \text { V. } 68 \text {, POZ2. 1978 } \\
& \text { FUKUSHIMA; EIICHI (CNC-4) } \\
& \text { GIBSON, A. A. V. } \\
& \text { SCOTT,T. A. }
\end{aligned}
$$

$50-43$

EVIDENCE FOR A RESONANCE IN

E+ -H S-WAVE SCATTERING.

PHYS. REV. LETT

V. 40: P. 3 $3-5$. 1978

DOOOLEN, GARY D. (TD-2)

NUTTALL, J. $\mathrm{J}$.
WHERRY, C.

$50-44$

EXCHANGE IN LOW - ENERGY ELECTRON MOLECULE SCATTERING. FREE - ELECTRON GAS MODEL EXCHANGE POTENTIALS AND APPLICATIONS TO E-HYDROGEN AND

E-NITROGEN COLLISIONS.

PHYS. REV. $17 \AA$ 18-38. 1978.

MORRIŚSN, MICHAEL A $(T-12)$

COLLINS, LEE A. (T-4)

$50-45$

EXCITONS IN GALLIUM MONOSELENIDE.

BULL. AM PHYS. SOC.,

V.23, P. 426. 1978 .

ALDRICH, CHARLES H. (M-6)

$50-46$

EXISTENCE OF THE DIELECTRIC CONSTANT IN

FLUIDS OF NONLINEAR RIGID POLAR

MOLECULES

J. CHEM. PHYS

V.68 RAMSHAW, JOHN D. 51998 -

$50-47$

EXTREME BACK ANGLE NEUTRON

DEUTERON ELASTIC SCATTERING

AT 794 MEV.

PHYS. REV.

V. 17 C BONNER $671-5$. 1978 (P-DOR)

SIMMONS JAMES E. (P-DOR)

EVANS, MICHAEL L: (Q-1)

GLASS, G. (P-DOR)

HIEBERT J. C.

JAIN, MAHAVIR (Q-1)

NORTHCLIFFE, LEE CONRAD

BJORK, C. W.

RILEY'PA PIS, CONSTANTINE G.
$50-48$

FRAME TRANSFORMATION RELATIONS AND

MULTIPOLE TRANSITIONS IN SYMMETRIC

POLYATOMIC MOLECULES.

REV. MOD. PHYS.

V. 50 HARTE $37-83.1978$.

PATTERSON, C.W. ( $T-7$ )
DA PAIXAO; FERNANDO J.

$50-49$

GAMMA SPECTRA FROM URANIUM -233

URANIUM -235 AND PLUTONIUM -239

1978: 13P. (LA-7451-MS)

BENDT, PHILIP J. $(\mathrm{P}-2)$

50-50

GENERALIZED LANGEVIN THEORY OF GAS / SOLID - SURFACE DYAMICS. A FORMULATION FOR THERMAL DESORPTION.

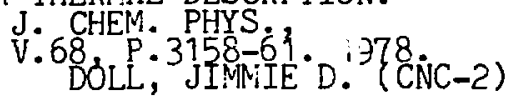

50-51

HIGH RESOLUTION SPECTROSCOPY OF THE OSMIUM TETPAOXIDE STRETCHING FUNDAMENTAL AT 961 CM $/$ SUP $-1 \%$.

$$
\begin{aligned}
& \text { J. CHEM. PHYS. } 1513-21.1978 . \\
& \text { MC DOWNELL ROBBIN S: (AP-4) } \\
& \text { RADZIEMSKI, LEON J., JR. (AP-4) } \\
& \text { FLICKER, HERBERT J.' (AP-DOT) } \\
& \text { GALBRAITH, HAROLD W }(-12) \\
& \text { KENNEDY, R. CRAIG (AP-3) } \\
& \text { NERESON' NORRIS G. (AP-4) } \\
& \text { KROHN, BURTON J' }(\mathrm{T}-12) \\
& \text { KING, JOE DAVID (AP-4) } \\
& \text { FOX, KENNETH }
\end{aligned}
$$

$50-52$

HIGH-J ASSIGNMENTS IN THE 10.5

MICROMETER SULFUR HEXAFLUORIDE
SPECTRUM. IDENTIFICATION OF THE

SPECTRUM. IDENTIFICATION OF THE

$P(12)$ AND $P(22)$.

OP. 2 PETT. $97-9.978$.

$M C$ DOWELL, ROBIN S. (AP-4)

GALBRAITH, HAROLD W. (T-12)

CANTRELL, CYRUS D., III $(T-12)$

NERESON,' NORRIS G.' (AP-4)

MOULTON, PETER F.

HINKLEY, E. DAVID

$50-53$

IMPROUED CALCULATION OF THE

MUONIC HELIUM LAMB SHIFT.

$$
\begin{aligned}
& \text { V.18A, P. 324-7. } 1978 . \\
& \text { RINIEER, G. GEORGE A., JR. (L-6) }
\end{aligned}
$$


$50-54$

IMPROVED PAULI HAMILTONIAN FOR LOCAL

POTENTIAL PRCRLEMS.

V. $18 \dot{8 B}, \stackrel{R E V}{\mathrm{P}} \cdot 2701-11.1978$.

WOOD JOHN H. (CMB-5)

$50-55$

INFRARED SPECTROSCOPY IN SUPERSOIIC

MOLECULAR BEAMS. NU 3 BAND OF SULFUR

HEXAFLUORIDE AT 10.6 MICROMETERS.

APPL. SPECTROSC.

V. 32. P. 287-9. j978-i)
KINI, KYU C. (AP-4)
HOLLAND, REDUS F- AF-4)
FILIP, HENRY (AP-4)

$50-56$

INHIBITION OF HOMOGENEOUS CONDENSATION OF SULFUR HEXAFLUORIDE BY VIBRATICHAL EXCITATION.

CHEM. PHYS. LETT.

KIM "KYU C (AP-4)

FILIP, HENRY (AP-4)

$50-57$

INTER-VALENCE-BAND ABSORPTION

IN ELECTRON-HOLE DROPLETS.

$$
\begin{aligned}
& \text { PHYS. REV LETT. } \\
& \text { V. } 41 \text { P } 1249-53 \text {. } 1978 . \\
& \text { SILVER, RICHARD N }(T-11) \\
& \text { ALDRICH, CHARLES H. }(M-6)
\end{aligned}
$$

$50-58$

IMTERMCLECULAR POTENTIAL ENERGY

SURFACES FROM ELECTRON! GAS METHODS

III, ANGLE, DISTANCE, AND VIBRATIONAL

DEPENDEIICE OF THE ARCON -

CAKBON MONOXIDE INTERACTION.

$$
\begin{aligned}
& \text { J. CHEM. PHYS } 1978 . \\
& \text { BARKER GREGORY A. (T-12) }
\end{aligned}
$$

$50-59$

INVESTIGATION OF THE MUITIPLE - PHOTON DISSOCIATION OF SULFUR CHLORIDE

PENTAFLUORIDE WITH A REAL TIME

$$
\text { CILORINE - ATDN DIAGNOSTIC. }
$$

J. CHEM. PHYS.

$$
\begin{aligned}
& \text { V.59 P. } 1196-\dot{9} \text { ' } 1978 . \\
& \text { LYMAN, JOHN L. (AP-3) }
\end{aligned}
$$

$50-60$

INVESTIGATTON OF URANIUM PLASMA

EMISSION FROM 1050 TO 6000 ANGSTROMS.

LASL, 152P. THESIS, UNIV.

1977. (LA-J101-T)
$50-61$

IOHIZATJON ENERGY LOSS OF

RELATIVISTIC ELECTRONS IN!

THIN SILICON DETECTORS.

PHYS. REV. LETT

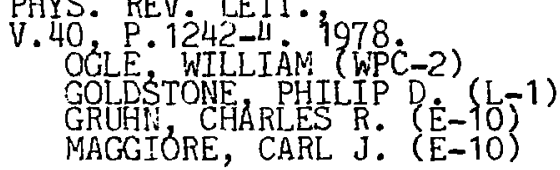

$50-62$

LASER - TNDUCED TIME - RESCLVED EMISSION OF ELECTRONICALLY - EXCITED URANIUM

HEXAFLIIORIDE.

CHEM. PHYS. LETT

V. $54, P .554-6.1978$.

WAMPLER FRED B: (AP-3)

OLDENBORG RICHARD C $(A P-3)$

RICE, WALTER $W$. (AP-2)

$50-63$

LASER INDUCED FLUORESCENCE OF GASEOUS URANIUM HEXAFLUORIDE IN THE A-X BAND

J. CHEM. PHYS.

V:69, P. $2181-7$ : 1978 OLDENBORG RICHARD C. (AP-3) RICE, WALTER W. (AF-2)
WAMPLER, FRED B. (AP-3)

$50-64$

LIFETIME AND OUENCHING RATE

CONSTANTS FOR KR (2) F AND KR(2). APPL. PHYS. LETT. V. 32, P. $649-5 \%$. 1978 GUIGLEY, G. P. $(\AA \dot{P}-3)$ HUGHES, WILLIAM M. (Q-8)

$50-65$

LIFETTME SATUFATION OF SULFUR DIOXIDE (3B1) MOLECULES. EVALUATION OF CHEVITCAL AND PHYSICAL QUENCHING CHANNELS IN ALKANES.

$$
\begin{aligned}
& \text { J. CHEM. PHYS } \\
& \text { - WAMPL } 2569-73.1978 \text { FRED } \\
& \text { OLDENBORG, KICHARD C. (AP-3) } \\
& \begin{array}{l}
\text { RICE, WALTER W. } \\
\text { KARL, ROBERT } \\
\text {. AP-2 }
\end{array}
\end{aligned}
$$

$50-66$

LINKED - CLUSTER PERTURBATION THEORY FOR CLOSED AND OPEN - SHELL SYSTEMS. DERIVATION OF LFFECTIVE

PI - ELECTRON HAMILTONIANS.

ELECTRONIC STRUCTURE AND

PROPERTIES OF POLYMERS 1977.

NATO ADVANCED STUDY INSTITUTE,

THEORY OF POLYMERS, P': 137-67.

D. REIDEL, 1978 BRD H. (T-11) 
$50-67$

LOCAL METHOD FOR DIGITALLY

RESTORING MOTION - BLURRED IMAGES.

IEEE, TRANS. ACOUST SPEECH,

P.256-63. 1978 .

HYMAN JAMES M.

$50-68$

LOW - LYING STATES OF ARGON +

KRYPTON + XENON +. I. POTENTIAL

CURVES WITH AND WITHOUT SPIN -

ORBIT COUPLING

J. 6 CHEM P. POYS 14.1978

WADT, WILLARD R. (T-12)

$50-69$

LOW ENERGY E-DIATOMIC MOLECULAR

SCATTERING. AN EXACT STATIC -

EXCHANGE CLOSE-COUPLING METHOD.

BULL. AM. PHYS SOC.

ROBLLINS, LEE A. (T-4)

50-70

LOW ENERGY E-MOLECULAR ION

SCATTERING. STATIC EXCHANGE AND

COUPLED ELECTRONIC STATE

CALCULATIONS.

BULL. AM ¿PHYS SOC. ,

COLLINS. LEE A. $(\mathrm{T}-4)$

$50-71$

MEASUREMENT AID MODEL INDEPENDENT

ANALYSIS OF THE X RAYS OF

SAMARIUM -152 .

PHYS. REV.

V. 18 Y

HOEHN' MARTHA V. $\left(\begin{array}{l}P-2 \\ P-2\end{array}\right)$

STEFFEN, ROLF M.

MO-72

EMISSION OF A TWO PHOTON EXCITED

AMMONIA LASER.

$$
\begin{aligned}
& \text { J. APPL. PHYS. } \\
& \text { V. } 49 \text {, } 2983 \text { \% } 978 \text {. } \\
& \text { FLICKER, HERBERT J (AP-DOT) } \\
& \text { HOLLAND, REDUS F. (AP-4) } \\
& \text { BISCHEL, W. K. } \\
& \text { PLUMMER, H. } \\
& \text { RHODES, CHARLES K. }
\end{aligned}
$$

$50-73$

MEASUREMENTS OF SOME LINE POSITIONS AND STRENGTHIS IN THE NU -4 REGION OF SULFUR HEXAFLUOFIDE.

$$
\begin{aligned}
& \text { J. CHEM. PHYS } \\
& \text { V.69 P } 1764-9 \text { ' } 1978 . \\
& \text { PERSON WILLIS B }(T-12) \\
& \text { KIM, KYU C. (AP-4) }
\end{aligned}
$$

$50-74$

WECHANISM AND RATES OF ROTATIONAL RELAXATION OF CARBON DIOXIDE (OOT) IN HELIUM AND ARGON.

$$
\begin{aligned}
& \text { J. CHEM. PHYS } \\
& \text { V.69 P } 2823-33.1978 . \\
& \text { PRESTON, PICHARO K. } \\
& \text { PACK, RUSSELL T. (T-12) }
\end{aligned}
$$

\section{$50-75$}

METHOD OF GENERALIZED COORDIIIATES AND AN APPLICATION TO RAYLEIGH-TAYLOR IMSTABILITY

$$
\begin{aligned}
& \text { PHYS. FLUIDS, } \\
& \text { V.21 P.736-44. 1979. } \\
& \text { DIENES, JOHN K. }
\end{aligned}
$$

$50-76$

MOBILITY OF ALPHA PARTICLES IN

PHYS. REV.

V. $18 \dot{A}$, P. $1009-11,1978$.

WADEHRA, JOGINDRA ( $\dot{T}-4)$

COHEN, JAMES S. $(T-12)$

$50-77$

WOLECULAR BEAM PHOTOIONIZATION

STUDY OF ACETONE AND ACETONE-D6.

J. CHEM!. PHYS, 1978 IROTT, WAYNE M. (C) BLAIS, NORMAND C. ( CNC-2) WALTEKS, EDWARD A.

MOLECULAR DYNAMICS OF SHOCK WAVES IN ONE - DIMENSIONALL CHAIMS.

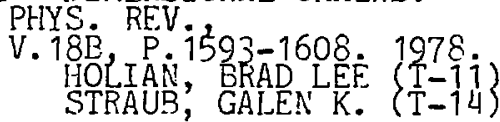

$50-79$

NITROGEN -15 MAGNETIC RESONANCE OF AQUEOUS IMIDAZOLF. AND Z INC (II) IMIDAZOLE COMPLEXES. EVIDENCE FOF HEXACOORDINATION!

$$
\begin{aligned}
& \text { INORG. CHEM } \\
& \text { ALEI, VCHANMED, JR. (CNC-4) } \\
& \text { WORGAN, LEON O }{ }^{(C N C-4)}
\end{aligned}
$$


$50-80$

NONLINEAR OPTICAL PROPERTIES OF

INDIUM ANTIMONIDE - HOT -ELECTRON

EFFECTS.

PHYS. REV.

V. $17 \dot{\mathrm{B}}, \mathrm{P}, 3336-45.1978$.

CANTRELL" CYRUS D., III (L-DOT)

SCOTT, J MARLAN $O$.

$50-81$

NOVEL ZERO TIME DETECTOR FOR HEAVY ION SPECTROSCOPY

NUCL INSTRUM. METHODS,

V.148, P. 503-9. 1978.

BOWMAN, J. DAVID (MP-4)
HEFFNER, ROBERT H. (MP-3)

$50-82$

NU /SUB 4/FUNDAMENTAL OF

THREE ISOTOPIC SPECIES OF

CARBON TETRAFLUORIDE.

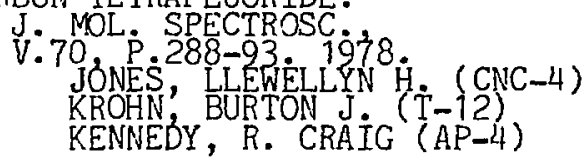

$50-83$

NUCLEAR CHARGE DISTRIBUTIONS

DEDUCED FROM THE MUONIC ATOMS

OF THORIUM -232 URANIUM -235
URANIUM -238 , AND PLUTONIUM -239.

PHYS. REV'

V. $17 C_{\text {P }} \cdot 1433-55.1978$.

CLOSE: DONALD A. $(Q-1)$

MALANIFY, JOHN J. $(P-11)$

DAVIDSON, JOHN P.

$50-84$

NUCLEAR MAGNETIC RESONANCE STUDIES

ON BACTERIAL DIHYDROFOLATE

REDUCTASE CONTA INING METHYL-

BIOCHEMISTRY,

V. 17 , P. $2284-93.1978$.

COCCO LENNIE

LONDON, ROBERT E. (CNC-4)

MATWIYOFF, NICHOLAS A. (CNC-4)

$50-85$

ON MAGNETIC TRANSITIONS AND THE

INTERPRETATION OF THE PARTIAL WAVE

PARAMETER IN THE CS AND IOS

APPROXIMATIONS IN MOLECULAR

SCATTERING THEORY.

$$
\begin{aligned}
& \text { J. CHEM. PHYS. } \\
& \text { V. } 99 \text { P. } 4419-30.1978 . \\
& \text { KHARE, V. } \\
& \text { KOURI, D. J. } \\
& \text { PACK, RUSSELL T. (T-12) }
\end{aligned}
$$

$50-86$

ON THE CONSTRUCTION OF THE R-MATRIX FOR THREE-DIMENSIONAL REACTIVE ATOM - DIATOM SCATTERING.

APPLI $\bar{C} A T I O N$ TO $\mathrm{H}+\overrightarrow{\mathrm{H}}$.

$$
\begin{aligned}
& \text { TIC, } \\
& 1977 \text {. } 42 P \text {. MN (LA-UR-78-966) } \\
& \text { STECHEL, ELLEN B. } \\
& \text { WALKER, ROBERT B. (T-12) } \\
& \text { LIGHT, JOHN C. }
\end{aligned}
$$

50-87

ON THE INFLUENCE OF MOLECULAR STRUCTURE UPON THE COLLISIONLESS LASER PHOTODISSOCIATION OF

SULFUR HEXAFLUORIDE. MULTIPHOTON PROCESSES, 1977 ' INTERNATIONAL CONF , UNI - OF J.H. EBERIY ED. WILEY, 1978 . $541.35 / \mathrm{Mg} 1 \mathrm{P}$ ) CYRUS D. III (T-12) GALERAITH, HAROLD W. (T-12) ACKERHALT, JAY R. (T-12)

$50-88$

ON THE RATE AND ACTIVATION ENERGY OF THE BROMINE ATOM + BROMINE MOLECULE ATOM - EXCHANGE REACTION. CHEM. PHYS. LETT

V. 55, P. $424-7$. 1978. THOMPSON, DONALD L. (CNC-2)

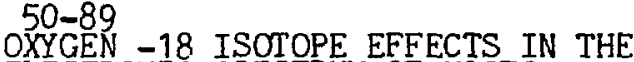
ELECTRONIC SPECTRUM OF UO2F2.

II. TEMPERATURE EFFECTS. INORG. NUCL. CHEM. LETT., V.14, P. 333-5. 1978. DE POORTER GERALD L. (AP-3)

$50-90$

PHENOMENOLOGICAL CONSTRAINTS ON BROKEN SUPERSYMMETRY.

PHYS. LETT.

V.78̈, $\mathrm{P}, 103-5.1978$.

GOLDMAN, TERRENCE J. (T-8)

$50-91$

PION - INDUCED SPALLATION OF COPPER ACROSS THE $(3,3)$ RESONANCE.

PHYS. REV ${ }^{\text {P. }}$ 1426-35. 1978.

ORTH, CHARLES J (CNC-11)

DROPESKY, BRUCE' J. (CNC-11)

WILLIAMS', ROBERT A. (CNC-11) GIESLER, GREGG CARL (CNC-11\} HUDIS, JEROME

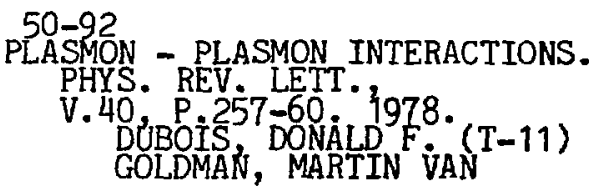


$50-93$

POLYHALIDE PHOTOFRAGMENT SPECTRA.

II ULTRAVTOLET PHOTODISSOCIATTON

DYNAMICS OF URANIUM HEXAFLLORIDE.

J. CHEM. PHYS
V. 8 P P. $4195-4201.1978$.
KROGER, PETER M.
RILEY, STEPHEN J
KWEI, GEORGE H. (CNC-2)

$50-94$

POSITIVE MUONS IN ALUMINUM. SELF-

TRAPPING AND DIEFUSION.

BULL. AM PHYS. SOC: ,

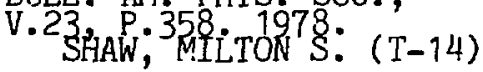

50-95 5 TAL CONSTANTS AND

DISSOC IATION PATHWAYS.

$$
\begin{aligned}
& \text { J. MOL. STRUCT. } \\
& \text { V.46, } .35-42 \text {. } 1978 . \\
& \text { JÓNES, LLEWELLYN }{ }_{\text {H. }}(\mathrm{CNC}-4) \\
& \text { RYAN, ROBERT R. (CNC-4) }
\end{aligned}
$$

$50-96$

POTENTIAL CONSTANTS OF CARBON

TETRAFLUOR IDE

$$
\begin{aligned}
& \text { J. } 6 \text { CHEM. PHYS. , }
\end{aligned}
$$

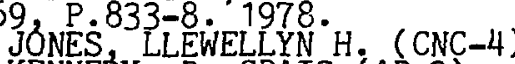

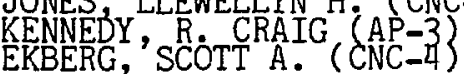

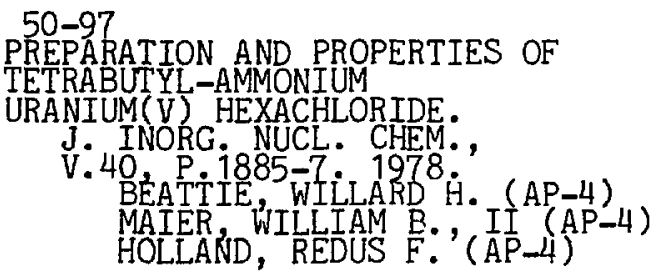

$50-98$

Q-BRANCH SPECTRUM OF THE 011DO-0000 TRANSITION OF 13C CARBON DIOXIDE.

J. MOL. SPECTROSC

$$
\begin{aligned}
& \text { V. } 6 \text { q P } 330-2 \text { 1978 } \\
& \text { FLISEELD, MERTERT J. (AP-3) }
\end{aligned}
$$

50-99

QUASICLASSICAL TRAJECTORY STUDY OF THE ENERGY TRANSFER IN CARBON

DIOXIDE - RARE GAS SYSTEMS.

J. CREM. PHYS. $455-72$ '. 1978.

SUZUKAWA, HENRY ${ }^{-}$JR. JR.

WOLFSBERG, MAX (CNC-2)
50-100

QUENCHING OF ELECTRONICALLY EXCITED URANIUM HEXAFLUORIDE

EMISSION BY SELECTED ORGANICS.

CHEM. PHYS. LETT.

V. 54 P. $557^{\circ}-9.928$

WAMPLER, FRED E. (AP-3)

OLDENBORG, RICHARD C $(A P-3)$

RICE, WALTER W. (AP-2)

50-101

QUENCHING OF ELECTRONICALLY - EXCITED URANIUM HEXAFLUORIDE BY SELECTED

INORGANICS

CHEY. PHYS. LETT.
V. 54 P $560-1978$.
WAMPLER FRED B. (AP-3)
OLDENBORG, RICHARD C $($ AP-3)
RICE, WALTER W. (AP-2)

50-102

QUENCHING OF ELECTRONICALLY EXCITED

URANIUM HEXAFLUORIDE.

AMERICAN CIIEMICAL SOCIETY.

NATTONAL MEETTNG. 1978 (MAR.)

$175 \mathrm{TH}$ ANAHEIM CA. ABSTRACTS OF

PAPERS, PHYS 144

WAMPLER, FRED E. (AP-3)

OLDENBORG RICHARD $C$-3) (AP-3)

RICE, WALTER W. (AP-2)

$50-103$

R MATRIX METHOD "APPLICATION

PHYSICS OF ELECTRONIC AND

ATOMIC COLLISIONS. $1977.10 \mathrm{TH}$

INTERNATIONAL CONFERENCE, PARIS.

PROC. ELECTRONIC AND ATOMIC

COLLISTONS P. $231-55$. ROBB, h. DEREK $(\dot{T}-4)$

$50-104$

R-MATRIX METHOD. APPLICATIONS

TO ELECTRON - MOLECULE COLLISIONS.

PHYSICS OF ELECTRONIC AND

ATOMIC COLLISIONS. $1977.10 T \mathrm{H}$

INTERNATTONAL CONFERENCE PARIS.

PROC. ELECTRONIC AND ATOMIC

COLLISIONS P. 257-69. SCHNEIDER,' BARRY' $(\mathrm{T}-12)$

$50-105$

R-MATRIX SOLUTION OF COLPLED

EQUATIONS FOR INELASTIC SCATTERING. J. 69 PHEM. PHYS. $13518-31.1978$. STECHEL, ELLËN B. WALKER, ROBERT B. (T-12) LIGHT, JOHN C. 
$50-106$

RADIATIVE LIFETIME AND QUENCHING

OF KRYPTON FLUORIDE.

APPL. PHYS. LETT.

QUIGLEY, G: P. (AP-3)

HUGHES, WILLIAM M. $(Q-8)$

$50-107$

RAPID ACCURATE CALCULATION OF

JWKE PHASE SHIFTS.

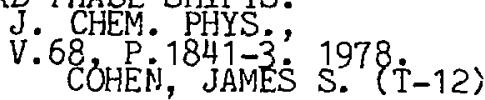

$50-108$

RATE COEFFICIENTS AND INELASTIC MOMENTUM TRANSFER CROSS SECTIONS FOR ELECTRONIC EXCITATION OF NITROGEN BY ELECTRONS.

J. APPL. PHYS.
V. 49 PARTWRIGHT, DAVID $197 .(T-12)$

$50-109$

RELATIVISTIC AND NONRELATIVISTIC

EFFECTIVE CORE POTENT IALS FOR XENON.

APPLICATIONS TO XENON FLUORIDE,

XENON AND XENON +.

V. CHEM:PHYS: 1752 : 1978.

WADT, WILLARD R. (T-12)

KAY P PUTS REREY

$50-110$

RELATIVISTIC FFFECTS IN AB INITIO

EFFECTIVE CORE POTENTIALS FOR MOLECULAR CALCULATIONS. APPLICATIONS TO THE URANIUM ATOM.

$$
\begin{aligned}
& \text { J. CHEM. PHYS } \\
& \text { V. } 68 \text { P P } 2386-97.1978 . \\
& \text { KAHN, LUIS R. } \\
& \text { HAY P. JEEREY (T-12) } \\
& \text { COWAN, ROBERT D. (T-4) }
\end{aligned}
$$

$50-111$

RESONANT PULSE EXCITATION LEADING

TO IONIZATTON.

PHYS. REV.

V. $17 \dot{A}$, P. $\dot{9} 33-9,1978$

ACKERHALT, JAY R. (T-12)

$50-112$

ROTATIONALLY AND VIBRATIONALLY INELASTIC SCATTERING IN THE ROTATIONAL IOS

APPROXIMATION. ULTRASIMPLE CALCULATION

OF TOTAL (DIFEERENTIAL INTEGRAL AND

TRANSPORT CROSS SECTIONS FOR

WONSPHER ICAL MOLECULES.

$$
\begin{aligned}
& \text { J. CHEM. PHYS } \\
& \text { V.68 P. } 1585-i 601.1978 . \\
& \text { PARKER GREGORY A } \\
& \text { PACK, RUSSELL T. (T-12) }
\end{aligned}
$$

$50-113$

SCATTERING THEORY AND EFFECTIVE MEDIUM APPROXIMATIONS TO HETEROGENEOUS

TIC

1977. 14P. MN (LA-UR-77-2063)

ALSO PUBLISHED IN:

ELECTRICAL TRANSPORT AND OPTICAL

PROPERTIES OF INHOMOGENEOUS MEDIA.

1977. CONF, OHIO STATE UNTV

PAPERS, P. 84-98. J.CC GARLAND, ED.

AIP GUBERNATIS, JAMES E. (T-11)

SELF 50 - CONSISTENT FIELD ELECTRONIC STRUCTURE CALCULATIONS FOR COMPRESSED MAGNESIUM OXIDE

J. PHYS. CHEM. SOLIDS,

V. 39 P P. $255-7$. $1978 \dot{1}$. (T-4)

$50-115$

SINGLE PHOTON TRANSITION MOMENTS IN EXCITED STATES OF SPHERICAL TOP

OPT. LETT

V. 3 GALBRAITH; HAROLLD W. (T-12)

50-116

SORTING OF A MURINE GRANULOCYTIC

PROGENITOR CELL BY USE OF LASER LIGHT SCATTERING MEASUREMENTS. TIC

$$
\begin{aligned}
& \text { TISP. MN (LA-UR-78-953) } \\
& \text { CISSER, JAN W. M. } \\
& \text { CRAMTIN SCOTT (H-10) } \\
& \text { MARTIN, JOHN C. (H-10) } \\
& \text { SALZMAN GARY C. }(H-10) \\
& \text { PRICE, BRANDON J. }(\mathrm{H}-10)
\end{aligned}
$$

ST-117 OF THE PH DEPENDENCE OF CARBON - 13 SHIFTS AND CARBON - CARBON COUPLING CONSTANTS OF (URANIUM CARBON -13)ASPARTIC AND

-GLUTAMIC ACIDS.

J. AM. CHEM. SOC.

V. $100, P .3723-9 \cdot 1978$ LONDON, ROBERT E. $\left\{\begin{array}{l}C N C-4 \\ \text { WALKER, THOMAS EN }\end{array}\right\}$ KOLLMAN VICTOR H. $(\mathrm{H}-11)$ MATWIYOFF, NICHOLAS A. (CNC-4)

50-118

STUDY OF THE NICKEL-59 (TRITON, PROTON) NICKEL-61 REACTION. PHYS REV $\mathrm{P}$.ंड $11-19.1978$. NAWN,
FLYNN, EDWARD R. $(\mathrm{P}-7)$
HANSON,
RAMAN
DAVID L. 
$50-119$

STUDY OF USES OF A PROTON

STORAGE RING.

LASL, 42P (LA-7490-MS) NAGLE, DARRAGH E. (MP-DO) COMP.

$50-120$

SU $(2 S+1)$ TIMES ZETA (R) AND

SPIN STATISTICAL WEIGHTS FOR

TETRAHEDRAL XY (4), TRICONAL

BIPYRAMIDAL XY (5),

OCTAHEDRAL XY (6).

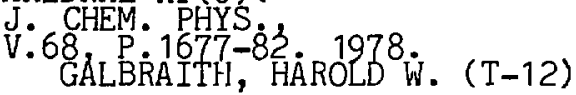

50-121 MECHANICS OE ELECTRONS AND HOLES IN SEMICONDUCTORS.

BULL. AM PHYS. SOC. ,

SILVER,
ALICHICH, CHARLES
H.

$50-122$

THEORETICAL PREDICTION OF

VIBRAT IONAL ENHANCEMENT FOR

DISSOCIATIVE CHARGE TRANSFER IN THE

IONIZED HELIUM HYDROGEN SYSTEM.

J. CHEM. PHYS. ' 1978

PRESTON, RICHARD K. (T-12)

THOMPSON, DONALD L: $($ CNC-2)
MC LAUGHLIN, DONALD R. (CNC-2)

$50-123$

THEORETICAL STUDIES OF THE LOW -

LYING ELECTRONIC STATES OF

EXTRAPOLATION TO INDIUM KRYPTON

AND THALLIUM KRYPTON.

J. CHEM. PHYS.

V.69, P. 2672-83 1978.

DUNNING, THOMAS H.; JR. ( CNC-2)

VALLEY, MARCY

TAYLOR, HOWARD $S$.

$50-124$

THEORY OF INELASTIC COLLISIONS BETWEEN

LOW - LYING EXCITED AND GROUND - STATE

NEON ATOMS

PHYS. REV

V. $17 \dot{A}$. P . $1343-56.1978,12)$

COL.LINS LEE A. $(\mathrm{T}-4)$

LANE, NEAL F.

$50-125$

THEORY OF SPIN - ORDER - DEPENDENT

HOPPING ACTIVATION ENERGY IN

NICKEL OXIDE.

BULL. AN . PHYS. SOC.

V.23 BRANDOW, BAIRD H. (T-11)
$50-126$

VARIATIONAL CALCULATIONS OF RESONANT STATES OF H2-

V. 11 PHYS. $\frac{\mathrm{B}}{\mathrm{P}} 95-54,1978$. COHEN, JAMES S. $(\mathrm{T}-12)^{-4}$

$50-127$

VELOCITY DEPENDENCE OF ANGULAR DISTRIBUT IONS IN GAS/SOLID -

SURFACE COLLISIONS. RELATIONSHIP

TO THE INTERACTION POTENTIAL.

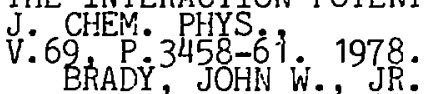

BRADY, JOHN W., JR. ( CNC-2)

DOLL, JIMMIE D' (CNC-2)

$50-128$

VIBRATIONAL ANALYSIS OF SULFUR HEXAFLUORTDE SPECTRA AND

AND IMPLICATIONS FOR COLLISSIONLESS

MULTIPLE PHOTON EXCITATION

MODELLING.

BULL. AM PHYS SOC.

GALERAITH, HAROLLD W. (T-12)

$50-129$

VIBRATIONAL ANHARM:ONICITY CONSTANTS FOR

SULFUR HEXAFLUORIDE. I. A 16 MICRON

DIODE LASER STUDY OE TRANSITIONS IN

THE NU -4 REGION.

$$
\begin{aligned}
& \text { J. CHEM. PHYS } \\
& \text { V. } 69 \text { PER } 2117-2 S^{2} 178, \\
& \text { KIM, KYU C. (AP-4) }
\end{aligned}
$$


$51-1$

ADJOINT MONTE CARLO TREATMENT OF

THE EQUATIONS OF RADIATIVE

TRANSFER FOR POLARIZED LIGHT.

$$
\begin{aligned}
& \text { J. COMPUT P PHYS. } 1978 . \\
& \text { CARTER, IELAAND L. (TD-6) } \\
& \text { HORAK, HENRY G. ( J-10) } \\
& \text { SA NDFORD, NAXWELL T. }(J-10)
\end{aligned}
$$

$51-2$

ANOMALOUS CEPHEID MASSES.

SKY TELESCOPE,

V.55, P. T15-8: 1978 .
COX, ARTHUR N. (T-DOT)

CEPHEID MASSES AND CEPHEID WINDS.

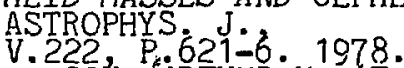

COX, ARTHUR N. (T-DOT)

MICHAUD, GEORGES

HODSON, STEPHEN W. (T-DOT)

$51-4$

CHARACTERISTIC ELECTRON VARIATIONS ACROSS SIMPLE H!ICH-SPEED SOLAR WIND STREAMS

$$
\begin{aligned}
& \text { J. GEOPHY . RES. ' } 1978 . \\
& \text { FELDMAN WILIIAM C: }(P-4) \\
& \text { BAME SAPUEL J, JR. }(P-4) \\
& \text { GOSLING, JOHN T? }(P-4)
\end{aligned}
$$

$51-5$

CHARACTERISTICS OF ENERGETIC

ELECTRONS IN THE VICINITY OF

THE BOUNDARY LAYER AT VELA OREIT.

J. GEOPHYS. RES., 1978.

PALMER, IATI D.

HONES, EDWARD W., JR. (P-4)

$51-6$

CHARACTERISTICS OF LATE - TIME

STRIATIONS OBSERVED DURING

OPERATION STRESS.

TIC

$$
\begin{aligned}
& \text { 8. 14P. MN (LA-UR-77-2835) } \\
& \text { WOLCOTT, JOHN H. }(\mathrm{J}-10) \\
& \text { EASTMAN TIMOTHY E. } \\
& \text { FITZGERÁLD, THOMAS JOSEPH }
\end{aligned}
$$

51-7

COMMENT ON "ON HOT TENUOUS PLASMAS, FIREBALLS, AND BOUNDARY LAYERS IN THE EARTH'S MAGNETOTAIL BY L. A. FRANK K. L. ACKERSON AND $\dot{R}$. P. LEPPING.

J. GEOPHYS. RES.

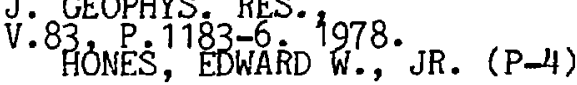

DETECTTION OF NUCLEAR EXPLOSIONS FROM SATELLITES AND SPACE PHYSICS RESEARCH.

$$
\begin{aligned}
& \text { LASL } \\
& \text { MINI-REVIEN, 1977. 4P. } \\
& \text { (LAS-77-222) } \\
& \text { CONNER, JERRY P. (P-4) }
\end{aligned}
$$

DOUBLE - MODE CEPHEID PERIOD RATIOS FROM LINEAR AND NONLINEAR THEORY .

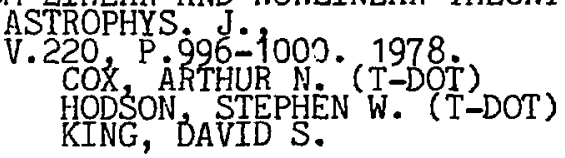

$51-10$

EFFECTS OF A LONG ENTRANCE APERTURE UPON THE AZIMUTHAL RESPONSE OF SPHERICAL

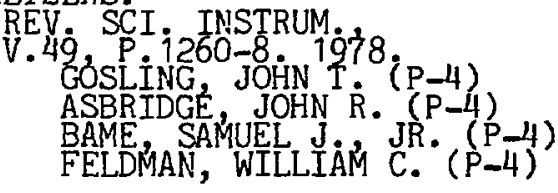

$51-11$ EFFECTS OF ROTATION AND CONVECTION ON DOUBLE MODE AND BUMP CEPHEIDS. ASTROPHYS. J. 2 . 1978.
V. 22 3 . P 982 .
DEUPRE, ROBERT G: (J-15)

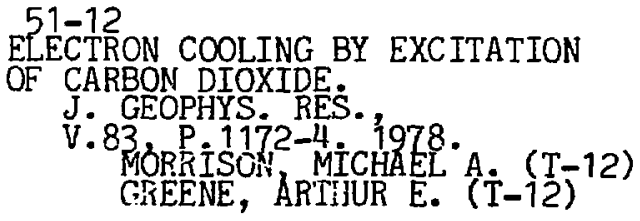


$51-13$

ELECTRON HEATING WITHIN INTERACTION ZONES OF STMPLE HIGH-SPEED SOLAR

J. GEOPHY S. RES.

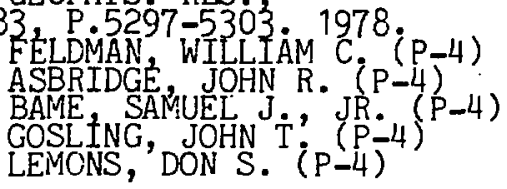

51-14

ENERGETIC PLASMA IONS WITHIN THE

EARTH'S MAGNETOSHEATH.

$$
\begin{aligned}
& \text { GEOPHYS RES. LETT., } \\
& \text { ASBRIDGE JOHI R. (P-4) } \\
& \text { GOSLING, JOHN T } \\
& \text { SCKOPKE, N. }
\end{aligned}
$$

$51-15$

ENHANCEMENT OF SOLAR CORONA AND

COMET DETAILS.

$$
\begin{aligned}
& \text { OPT. ENG } \\
& \text { V. } 17 \text { P } 6 \text { 1-5. } 1978 \text {. } \\
& \text { MATUSKA, WALTER ( J-15) } \\
& \text { JANNEY, DONALD H (N-8) } \\
& \text { FARRELL, JOHN ALDEN (AT-2) } \\
& \text { KELLER,' CHARLES F. (J-15) }
\end{aligned}
$$

$51-16$

EVOLUTION OF THE SOLAR "CONSTANT" .

URANIUM MILL TAILINGS

MANAGEMENT 1978 SYMP 11 FORT

COLLINS, CO. PROC. P. $111-39$;
NEWMAN, MICHAEL'J. (T-DOT)

$51-17$

FINAL REPORT. NEW RESEARCH INITIATIVES PROGRAM, PROJECT W-219, CHEMISTRY IN COMET COMAE.

1978 : 6P. (LA-7096-MS)

HUEBNER, WALTER F. (T-4)

$51-18$

FLUCTUATIONS OF QUASARS AND

OTHER ASTRONOMICAL SOURCES.

INITIATIVES PROGRAM W-210.

LASL,

1978: 28P. (LA-7092-MS)

$51-19$

HELIUM ABUNDANCE IN GALACTIC

GLOBULAR CLUSTERS

PUBL. ASTRON. SOC. PACIFIC,

V. 90 : P.53-6: 1978

DEUPREE ROBERT' G. (T-DOT)

EOLL JOHN G. (TD-2)

HODSON, STEPHEN W. (T-DOT)
51-20

HIGH - RESOLUTION ENERGETIC PARTICLE MEASUREYENTS AT 6.6 RE, 2 HIGH -

ENERGY PROTON DRIFT ECHOES.

$$
\begin{aligned}
& \text { J. GEOPHY S RES. } \\
& \text { V.83, NO.A 10 P P } 4857-62.1978 . \\
& \text { BELIAN, RICHARD D }(P-4) \\
& \text { BAKER, DANIEL N. (P-4) } \\
& \text { HIGBIE, PAUL R. (P-4) } \\
& \text { HONES, EDWARD W., JR. (P-4) }
\end{aligned}
$$

$51-21$

HIGH - RESOLUTION ENERGETIC PARTICLE MEASUREMENTS AT 6.6 RE, 1. ELECTRON MICROPULSATIONS

$$
\begin{aligned}
& \text { J. GEOPHYS. RES. 4851-5. } 1978 . \\
& \text { HIGBIE, PAUL } R \text {. }(\mathrm{P}-4) \\
& \text { BELIAN, RICHARD D, }(P-4)
\end{aligned}
$$

$51-22$

HIGH - RESOLUTION ENERGETIC PARTICLE MEASUREMENTS AT 6.6 RE, 3 . LON ENERTY E TERM SUBSTORM PREDICTIONS.

J. GE.OPHYS. RES. $4863-8.1978$.

BAKER, DARIEL N. (P-4)

HIGBIE, PAUL R. ( $P-4)$ BELIAH, RICHARD D. $(\dot{P}-4)$

$51-23$

INTERSTELLAR COLONIZATION.

J. BR. INTERPLANET. SOC. ,

V. 31 JO̊NES, ERIC 1978 .

ION-24 ACOUSTIC - LIKE INSTAEILITIES

IN THE SOLAR WIND

J. GEOPHYS. RES. ,

V. 83 GARY, $2504-10 .{ }^{2} 1978$.

ISEE-C SOLAP WIND PLASMA EXPERIMENT. IEEE TRANS. GEOSCI ELECTRON. , BAME SAMUEL J JR: $(P-4)$ ASERTDGE, JOHN R. $(P-4)$ FELTHAUSER, HARRY E

HAWK, H. L.

CHAVEZ, J. 
51-26

ISEE-1 AND ISEE-2 FAST PLASMA

EXPERIMENT AND THE ISEE-1 SOLAR

WIND EXPER IMENT

IEEE TRANS. GEOSCI. ELECTRON.,

V.GE-16, P.216-20. 1978.

BAME' SAMUEL J. JR. $(\mathrm{P}-4)$

ASERTDGE JOHN R. $(P-4)$

GLORE, JAMES $P$. $(P-4)$

PASCHMANN, GOTZ

HEMMERICH', $\mathrm{P}$.

LEHMIANN, k.

ROSENBAUER, $\mathrm{H}$.

$51-27$

KIMBERLITES ON MARS.

KIMBERLITE CONFERENCE. 1977.

2ND INTER RATIONAL SANTA FE, MM.

THE CONFERENCE, 1977.

MC GETCHIN THOMAS $R_{2}(G-6)$

$51-28$

LAGOPEDO - TWO F-REGION IONOSPHERIC DEPLETION EXPERIMENTS.

197

$$
\begin{aligned}
& \text { PONGRATZ, MORPIS B. (J-10) } \\
& \text { SMITH, GORDOH M. (J-10) } \\
& \text { SUTHERLAND, C. DEXTER (J-10) } \\
& \text { ZINN, JOHN' (J-10) }
\end{aligned}
$$

\section{$51-29$}

SOLAR WIND PROPERTIES. IMP 6,7

AND 8 RESULTS.

J. GEOPHYS: RES.

V. 83. NO.A5, P. $1177-89 \cdot 1978$.

ASBRIDGE, JOHN R. ( $P-4)$

BAME SAMUEL J ' JR. (P-4)

GOSLTNG, JOHN T'. ( $\mathrm{P}-4$

\section{$51-30$}

MAGNETOSHEATH DISTORTION OF

PITCH AIILE DISTRIBUTIONS OF

SOLAR PROTONS.

J. GEOPHYS. RES.

FILEIE, PAUL $\dot{R} .(\mathrm{P}-4)$

MODEL OF COMET COMAE, I. GAS PHASE CHEMISTRY IN ONE DIMENSION.

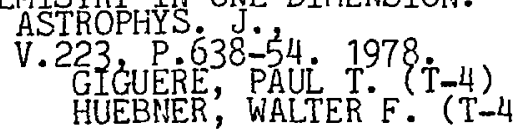

$51-32$

MODEL OF THE FORMATIOH OF ELLIPTICAL GALAXIES

ASTROPHYS, AND SPACE SCI., BROWN, WILBUR $\mathrm{K}$. (TD-7)

$51-33$

OBSERVATIONS OF TWO DISTINCT

POPULATIONS OF BOW SHOCK IONS IN THE UPSTREAM SOLAR WIND.

GEOPHYS. RES. LETT. AOSLING JOHN T: $(\mathrm{P}-4)$ ASME, SAMUEL J., JR. $(\mathrm{P}-4)$ PASCAMANN,

OBSERVATC

SERVATORY REPORT.

BULL. AM ASTRON SOC.,

COX, ARTHUR N. (T-DOT)

$51-35$

PHOTOGRAPHS OF THE ORION NEBULA

IN HYDROGEN ALPHA HYDROGEN BETA

AND HELIUM I LAMBDA 10830.

ASTRON. ASTROPHYS

V. 67 P. $435-6.1978$

GO'W CHARLES E. ( $J-9)$

SANDFORD, MAXWELL T. ( J-9)

HONEYCUTT, R. KENT

$51-36$

PHOTOMETRIC STUDY OF THE M51

SYSTEM.

ASTROPHYS. J.

SUPPL- SERIES V. 38 ,

BURKHEAD, MARTIN S. (J-15)

51-37

PLASMA SHEET PRESSURE ANISOTROPIES.

$$
\begin{aligned}
& \text { J. GEOPHYS RES.' } \\
& \text { V. } 83 \text {, } 3166-72.1978 . \\
& \text { STILES, GARDINER S. } \\
& \text { HONES, EDWARD W., JR. }(P-4) \\
& \text { BAME SAMUEL J.' JR. }(P-4) \\
& \text { ASBRTDGE, JOHN R. }(P-4)
\end{aligned}
$$

POWER SPECTRUM OF THE SOLAR WIND SPEED FOR PERIODS GREATER THAN 10 DAYS.

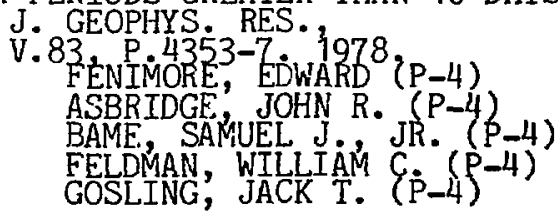




\section{1-39}

PROCESSES AT THE MAGNETOTAIL

BOUNDARY - COMMENTS ON ION HOT

BOUNDARY LAYERS' IN THE EARTH'S

MAGNETOTAIL ' BY L.A. FRAMK

K.L. ACKERSON, AND R.P. LEPPING.

J. 83 NO.A5, RES $2316-27.1978$. HONES, EDWARD W., JR. (P-4)

$51-40$

PULSATION MODAL BEHAVIOR OF CLASSICAL

CEPHEIDS IN THE HR DIAGGAM.

INTERNATIONAL ASTRONOMICAL UNION.

SYMPOSIUM NO.80. HR DIAGRAM.

1977 SYMPOSIUM, WASHINGTON, D.C.

PROCEEDINGS, P. $337-40$.

A. G. DAVIS PHILIP, ED.

COX, ARTHUR N. (T-DOT)

HODSON, STEPHEN W. (T-DOT)

$51-41$

REMARKABLE EXTENT OF THE

CIRCUMSTELLAR GAS SHELL SURROUNDING

BETELGEUSE.

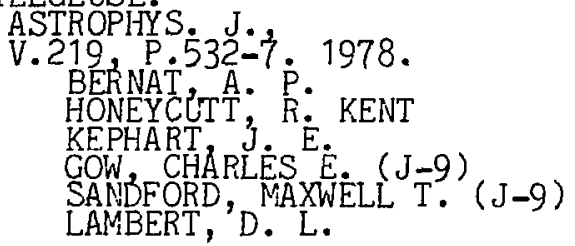

ASTROPHYS. J.

HONEYCUTT, R. KENT

KEPHART J E E.

SANDFORD, MAXWELL T. ( J-9)

LAMBERT, D. L.

$51-42$

SHOCK - ASSOCIATED ENERGETIC PROTON

EVENTS AT LARGE HELIOCENTRIC DISTANCES.

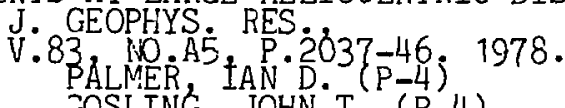

JOSLING, JOHN T. (P-4)

$51-42$

SOLAR OPACITY AND EQUATION OF STATE.

TIC. 27P. MN (LA-UR-78-196)

HUEBNER, WALTER F. $(\mathrm{T}-4)$

$51-44$

J. GEOPHY STRAM INTERFACES.

J. GEOPHYS. RES. ' 1978.

GOSLING, JOHN T $(\mathrm{P}-4)$

ASBRIDGE, JOHN R. (P-4)

BAME, SAMUEL J.'JR. JR $(\mathrm{P}-4)$

\section{$51-45$}

SOME CHARACTERISTICS OF PAPIDLY FLOWING MAGNETOTAIL PLASMAS - FURTHER COMMENTS ON 'ON HOT TENUOUS PLASMAS FIREBALLS, AND BOUHDARY LAYERS IN THE EARTH'S MAGNETOTAIL SY L.A. FRANK, K.L. ACKERSON, AND R.P. LEPPING. J. GEOPHYS. RES. V.83. P. 3358-64.'1978.j. (P-4)

$51-46$

SOME INITIAL SIGNS IN THE PLASMA SHEET J. GEOMAG. GEOELECTR., V. 30 HONES, ${ }^{191}$ EDWARD 1 i. , JR. (P-4)

$51-47$

SOME OBSERVATIONS OF A SUBSYNOPTIC SCALE DISTURBANCE. LASL,

1978: 12P (LA-7394-MS)

BARR, SUMNER (G-8)

CLEMENTS, WTLLIAM E, $(G-8)$

$51-48$

SUPERMOVA RADIO PULSE. SEARCHES

AND POSSIELE IMEROVENENTS IN

SENSTTIVITY

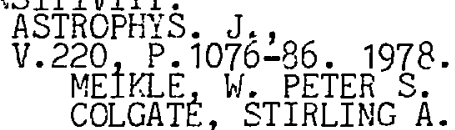

( T-DCT)

51-49

THEORETICAL LICHT AND VELOCITY CURVES FOK CEPHEID VAFIABLES.

LASL

1978: 13P. (LA-7599-ViS)

ADAMS, THONAS F. (J-15)

DAVIS, CECIL G. $(J-15)$

KELLER, CHARLES F. (J-15)

$51-50$

THEORETICAL MODELS OF 10-DAY

CEPHEIDS

ASTROPHYS. J.

V.221 P.929-36. 1978.
DAVIS, CECIL G. $(j-15)$
DAVISON, DAVID $K$.

$51-51$

THREE-DIMENSIONAL DYNAMICS CF PROTOSTELLAR EVOLUTIOR.

$$
\begin{aligned}
& \text { ASTRCPHYS, J., } \\
& \text { V.225,P. 1005-20. } 1978 \text {. } \\
& \text { COOK, THONAS L (J-O) } \\
& \text { HARLOW, FRANCIS H. }(\mathrm{T}-3)
\end{aligned}
$$


TORDO I POLAR CUSP BARIUM PLASMA

INJECTTON EXPERIMENT.

3. CEOPHYS. RES.

V. 83 P. $1565-75$.' 1978.

NESCOTT EUGENEM.

STENBAEK-NIELSEN, H. C.

JEFFRIES, ROBERT A $(\mathrm{J}-10)$
ROACH, WILLIAM H. $(\mathrm{J}-12)$

TRIPLE MODE CEPHEID MASSES.

A. VTROPHYS J.3. 1978.

COX, ARTHUR N. (T-DOT)

HODSON, STEPHEN W. (T-DOT)

$51-54$

UNUSUAL ASPECT OF SOLAR WIMD

SPEED VARIATIONS DURING SOLAR

CYCLE 20

J. GEOPHYS. RES.

V.82 P. $3311-4$ '1977. $19-4)$

ASBRIDGE, JOHN $\dot{R}$. $(P-4)$

BAME, SAMUEL J., JR. (P-4)

VIBRATIONAL POPULATIONS OF THE

EXCITED STATES OF NITROGEN

UNDER AURORAL CONDITIONS.

V.. GEOPHYS. RES. 1978.

CAKTWRIGHT, DAVID C. (T-12) 
ALGORITHM FOR THE DISCRETE REZONING

OF LAGRANGIAN MESHES.

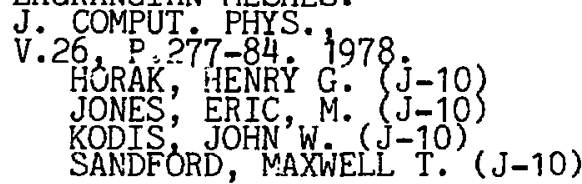

$52-2$

ANALYSIS OF A RESONANT - CAVITY

MEASUREMENT OF THE VELOCITY OF LIGHT.

STANFORD UNIV., CALIF

MICROWAVE LAB: '1956. $133 \mathrm{P}$

M.L. PEPORT NO. 343. (AFOSR TN-56-325) JANNEY, DONALD $\dot{H}$.

$52-3$

AMOMALOUS RESISTIVITY MAXIMA

IN SOME MAGNETIC SYSTEMS.

SOLID STATE COMMUN.

V. $26, P .831-4.1978$ '

HUANG, CHAO-YUAN (Q-10)

$52-4$

BREAKUP OF AN ACCELERATED SHELL

OWING TO RAYLEIGH - TAYLOR

INSTABILITY.

$$
\begin{aligned}
& \text { LASL, 13P. (LA-7291-MS) } \\
& 1978 \text { SUYDAM; BERGN R. (T-7) }
\end{aligned}
$$

$52-5$

BROADENING OF A SPECTRUM LINE BY

FINITE SPECTROMETER RESOLUTION.

1978

ENGLEMAN, ROLF, JR. (CMB-1)

$52-6$

CÁSE OF THE PHANTOM PHASES IN

SOLID HYDROGEN.

J. LOW TEMP: PHYS.

V. 31 P. $423-8.197^{\circ}$

MÍLLS, ROBERT L. (Q-10)

CAT-7 PATTERNS.

$$
\begin{aligned}
& \text { LASL, } \\
& 1978 \text { : 4OP, (LA-7384-MS) } \\
& \text { CAMPBELL LAURENCE J (Q-10) } \\
& \text { ZIFF, ROBERT M. (Q-10) }
\end{aligned}
$$

CHEMICAL TRACERS IN HYBLA GOLD WORKING ROOM. FINAL REPORT.

LASL,

1978, 12P. (LA-7097-MS)

MILLIAN, BARBARA GERMAIN (J-9)

$52-9$

COST OF SPLITTING IN MONTE CARLO TRANSPORT.

LASL,

1978: 9P. (LA-7189-MS)

CVERETT, CORNELIUS J (TD-6)

52-10

DERIVED THERMODYNAMIC PROPERTIES

FOR FLUID N-T2 IN THE RANGE 75$300 \mathrm{~K}$ AND 2-20 KBAR.

LASL

$$
\begin{aligned}
& \text { (LA-7071-MS) } \\
& \text { LIEBENBERG DONALD H. }(\mathrm{Q}-10) \\
& \text { MILLS, ROBERT L. (CMB-5) }
\end{aligned}
$$

52-11

DIFFUSION COEFFICIENT DETERMINATION USING A SIZE VARIATION TECHNIQUE.

J. APPL. PHYS.
V. 49 P. $4583-5.1978$. STARK, WALTER A., JR. (CMB-3)

$52-12$

DIRECT MEASUREMENT OF CESTUM

ABSORPTION ISOBARS ON

POLYCRYSTALLINE TANTALUM.

J. APPL. PHYS 49 ' 1978.

COLLIER, ROBERT K. (Q-11)

JACOBSÓN, D. L.

DISTRIBUTED APERTURE EFFECT

IN LASER RODS WITH NEGATIVE LENSES. APPL. OPT.

V. 17. $1537-40,1978$. BAREES, :ORMAM P. (AP-2)

$52-14$

EFFECT OF ELECTRICAL STATE ON MECHANICAL AND ELECTRICAL

RESPONSE OF A FERROELECTRIC CERAMIC PZT $95 / 5$ TO IMPACT LOADING.

$$
\begin{aligned}
& \text { J. APPL. PHYS, } \\
& \text { V. } 49 \text { P P } 494-8: 1978 . \\
& \text { DICK JERRY JOEL (M-3) } \\
& \text { VORTMMAN, J. E. }
\end{aligned}
$$


$52-15$
ELECTRON TRANSPORT CODE

THEORETICA:. BASIS.

LASL,

1978. 8P. (LA-7227-MS)

DUBI, ARIE $(\mathrm{T}-1)$

$52-16$

EPK PARADOX REVISITED.

PHYS. REP.

V. $43.9 .49 \dot{9}-508 \cdot 1978$.

SCULEY, PHARLAN D." (L-DO) (L-DOT)

52-17

EQUATION OF STATE OF FLUID

TRITIUM DEDUCED FROM P-V -T AND

ULTRASONIC-VELOCITY MEASUREMENTS

ON HYDROGEN AND DEUTERIUM

TO $20 \mathrm{KBAR}$.

J. APPL: PHYS,

MILLS ROBERT 1978.

LIEEENBERG DONALD $H$ H
BRONSON, JOHN C. $(Q-10)$

$52-18$

EQUATION OF STATE OF FLUID N-D2 FRON,

P-V-T AND ULTRASONIC VELOCITY

MEASUREMENTS TO $20 \mathrm{KEAR}$.

$\mathrm{J}: 6 \mathrm{CHEM}_{\mathrm{P}}: 26 \mathrm{PHS}^{3}, \mathbf{8}: 1978$

MILLS ROEERT L (Q-10)

LIEBENBERG DONALD H H $(Q-10)$

$52-19$

EXACT WAVE - FUNCTION

NORMALIZATION CONSTANTS FOR THE

B TAMH Z - U/(COSH Z)-SQUARED

POTENTIAL AND POSCHL-TELLER

POTENTIALS.

PIYS. REV $1273-83.1978$.

NIETO; MICHAEL MARTIN (T-8)

$52-20$

FINITE DIFFERENCE METHODS IN

MULT I-DIMENSIONAL TWO - PHASE

FLOW.

TIC, $19 \mathrm{P} . \mathrm{MN}$ (LA-UR-77-2378)
TRAVIS; JOHN R. (T-3)

$52-21$

GEMINATE RECOMEINATION OF ALPHA PARTICLE EXCITED CARRIERS IN

LIQUID ARGON.

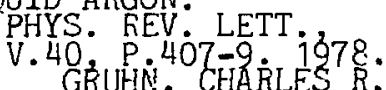

GRUH CHARLES R: (E-10)

EDMISTON, NICHAEL (E-10)
52-22

EXAMPLES OF FREE DRAINAGE OF NEWTONIAN AND NON-NEWTONIAN EILMS WITH TIME -

DEPENDENT RHEOLOGICAL PARAMETERS.

$$
\begin{aligned}
& \text { TIC } \\
& 1977 \text {. 25P. MN (LA-UR-77-2014) } \\
& \text { PENDERGRASS, JOHN H. (L-5) }
\end{aligned}
$$

HIGH -23 PRESSURE GASES IN

DIAMOND CELLS.

PHYS. LETT: $162-4.1978$. IEEBENBRG, DONALD H. ${ }^{(Q-10)}$
MILLS HOBERT L. $(Q-10)^{(2-10}$
BRONSON, JOHN C. $(Q-10)$
SCHMIDT, LEROY C. $(S D-5)$

$52-24$

HIGH BANDWIDTH RADIATION DIAGNOSTICS WITH SCINTILLATORS AND FIBER OPTICS

LASER AND ELECTROOPTICAL SYSTEMS.

1978. OSA/IEEE CONFERENCE,

SAN DIEGO, CA. DIGES I OF

TECHNICAL PAPERS, NO. TUGG4,

P. 20. OSA 1978 . GOLOB, JOHN E. E-3)

LIEBER, ALBERT $(J-14)$

LOONEY, LARRY D. $(\mathrm{J}-14)$

LYONS, PETER B. $(\mathrm{J}-14)$

FRANKS, L.

LUTZ S. $S . A$.

ROBICHAUD; R. E.

$52-25$

HIGH ENERGY NEUTRON DOSIMETRY

FOR THE FUSION PROGRAM.

REACTOR DOSIMETRY . 1977.

(OCT.) ASTM-EURATOM SYMP. 2ND,

PALO ALTO. PROC. DCSIMETRY

METHODS FOR FUELS, CLADDING,

AND STRUCTURAL MATERIALS.

P. 1059-68. (NUREG/CP-0004 V.3)

BARR DONALD W. $($ CNC-11)
NORRIS, ANDREW! E. (CNC-11)

$52-26$

HOMOGENEOUS NUCLEATION - $A$

PROBLEM IN NONEQUILIBRIUM QUANTUM

STATISTICAL MECHANICS.

LASL,

1978: 277P. THESIS, UNIV OF NM,

ALBUQUEROUE, 1978 ' (LA-7441-T) LARSON, ALVIN R. (L-5)

52-27

HYDRODYNAMIC APPROACH TO ELECTRON HOLE DROPLET NUCLEATION AND STABILITY. VHYS. REV. $3955-71,1978$. SILVER, RICHARD N. (T-11) 
$52-28$

HYDRODYNAMIC MODEL FOR ELECTRON HOLE DROPLETS

SOLID STATE COMMUN.

V. 25 SILVER, RICHARD $\dot{\mathrm{N}} .(\mathrm{T}-11)$

$52-29$

INITIIAL VALUE PROBLEM FOR RAVLEIGH TAYLOR INSTABILITY OF VISCOUS FLUIDS. PHYS. FLUIDS,

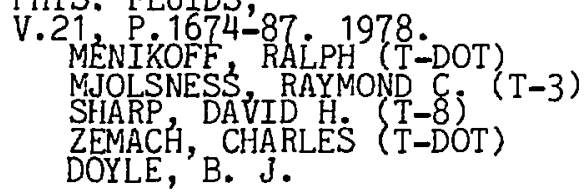

$52-30$

INTERACTION OF EXPLOSIVE - DRIVEN AIR SHOCKS WITH WATER AND PLEXIGLAS. LASL

1978: 22P. (LA-7454)

SLAUGHTER, IILTON D. (T-8)

OLINGER BARTON W. $(M-6)$

MADER CHARLES L.. $(T-14)$

BOWMAN , ALLEN L. (T-14)

$52-31$

INVESTIGATION OF HYPERSCALING IN THE CONTINUOUS SPIN ISINC MODEL.

BULL. AM. PHYS. SOC.,

23 P. 341.1978.

KAKER GEORGE Á., JR ${ }_{\text {KINCAD, JOHN M. }}^{(T-11)}$

$52-32$

JET TARGET INTENSE NEUTRON SOURCE. TIC77. 6P. MN (LA-UR-77-2398)

ALSO PUBLISHED IN:

ENGINEERING PROBLEMS OF FUSION

RESEARCH. 1977. SYMP. 7TH, KNOXVILLE, TN. PROC.; V.2; P. 1435-99.M. S. LUBELL ED. MEIER, KARL L. (P-14)

$52-33$

ASL NIOBIUM( 3 )GERMANIUM

CONDUCTOR DEVELOPMENT, OCTOBER 1JECEMBER 31, 1977.

1978, 12P. (LA-7204-PR)

MALEY, MARTIN P. $(Q-10)$ COMP.

\section{$52-34$}

ASL NIOBIUM(3) GERMANIUM CONDUCTOR WEVELOPMENT JANUARY 1-

1978. 22P. (LA-7356-Fin)

MALEY, MARTIN P. $(Q-10)$ COMP.
52-35

LIMB-DARKENING OPACITY EXPERIMENT USING A LASER-HEATED PLASMA.

1978, 13P. (LA-7484-MS) HOFFMAN, NELSON M. (J-15) MALE, JOSEPH. M. , JR. (J-15)

52-315 OF VALIDITY FOR THE VAVILOV ENERGY STRAGGLING CALCULATION

COMPUT. PHYS, COMMUN. , JARM IE, NELSSON (P-9) PINDZOLA, MICHAEL S. BICHSEL, 'H.

LOW-37 TEMPEFATURE PHYSICS AND

ENGINEERING QUARTERLY PROGRESS

OCTOBER 1-DECEMBER 31, 1977.

LASL, 50P. (LA-7249-PR)

KÉLLER; WILLIAM E. (Q-10) COMP.

52-38

LOW TEMPEKATURE PHYSICS AYD

ENGINEERING QUARTERLY PROGRESS

REPORT, JULY 1-MARCH 31, 1978.

1978: 43P. (LA-7374-PR)

KELLER, WILLIAM E. (Q-10) COMP.

52-39 39 MPEFATURE PHYSICS AND

ENGINEERING QUARTERLY PROGRESS

REPORT, APRIL 1-JUHE 30, 1978.

1978. 49P. (LA-7448-PR)

KELLER; WILLIAM E. $(Q-10)$ COMP.

$52-40$

LOW TEMPERATURE PHYSICS AND ENGINEERING

QUAFTERIY PROGRESS REPORT, JULY 1 -

SEPTEMBER 30, 1977.

LASL

1978: 35P. (LA-7134-PR)

KELLER, WILLIAM E. (Q-10) CORP.

$52-41$

MARGINAL DENSITIES OF RADIALLY

SYMMETRIC DENSITIES IN TWO AND

THREE DIMENSIONS.

I.ASL

1978: 18P. (LA-7283-MS)

EVERETT, CORNELIUS J (TD-6)

CASHWELL, EDMOND D. (TD-6) 
$52-42$

MOMENT GENERATED RADIATIVE

TRANSFER FUNCTIONS FOR RELATIVISTIC

MAXWELLIAN ELECTRONS.

J. QUANT. SPECTROSC. RADIAT. TRANSFEF

V. 19 P. $163-8.1978$.

WIENKE, BRUCE R. (T-1)

$52-43$

MUTUAL (ISOTHERMAL) DIFFUSION

IN BINARY HARD SPHERE MIXTURES.

THE EFFECTS OF THE ONSAGER

RECIPROCITY RELATIONS.

PHYS, LETT

KINCAID, JOHN M. $(\dot{T}-11)$

$52-44$

NEW METHOD OF SAMPLING THE KLEIN NISHINA PROBABILITY DISTRIBUTION

FOR ALL INCIDENT PHOTON ENERGIES

ABOVE 1 KEV (A REVISED COMPLETE

ACCOUNT).

LASL,

1978: 13P. (LA-7188-MS)

EVERETT, CORNELIUS J (TD-6)

CASHWELL, EDMOND D. (TD-6)

$52-45$

NOTE ON THE ROUNDOFF ERROR IN THE

NUMEROV ALGORITHM.

J. COMPUT, PHYS
FRIAR, JAMES L. $(\mathrm{T}-5)$

52-46

INEQUALITY (A KNAPSACK PROBLEM).

LASL

1978: 8P. (LA-7287-MS)

EVERETT CORNELIUS J. (TD-6)

$52-47$

NUMERICAL CALCULATION OF HIGH -

SPEED MULTIPHASE FLOW.

BULL. AM PHYS SOC .

$$
\begin{aligned}
& \text { V.23, P. 1006. } 1978 . \\
& \text { COOK THOMAS L. }(J-15) \\
& \text { DEMUTH, RUTH B. }(T-3)
\end{aligned}
$$

HARLOW', FRANCIS $1 . .(T-3)$

$52-48$

NUMERICAL SIMULATION OF HYDRODYNAMIC

IMPACT LOADS ON CYLINDERS.

NUCL. SCI 1 ENG. 1978.

NICHOLS, BILL D. $(T-3)$
$52-49$

OBSERVATION OF VARIABLE MODES OF

HEAT TRANSFER TO LIOUID HELIUM

SUPERCONDUCTIVITY BY CURRENT.

EULL. AM. PHYS. SOC.,

WEINSTOCK, H.

OVERTON, WILLIAM

C., JR. (Q-10)

$52-50$

OBSERVATION OF WIDE N-S

INTERFACES DURING DESTRUCTION OF SUPERCONDUCTIVITY BY

CURRENT.

BULL. AM, PHYS, SOC.,

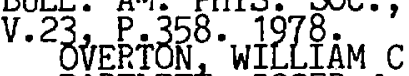

BARTLETT, ROGER J

WEINSTOCK, $\mathrm{H}$.

52-51

ON THE EXISTENCE OF COMPLEX RELAXATIO ENGTHS IN AN ABSORBING MEDIUM.

TRANSP. THEORY AND STAT.

PHYS., V.7, P.25-32.

LARSEN, EDWARD W. (T-1)

PERFORMANCE CHARACTERISTIC OF DISCHARGE EXCITED EXCIMER

LASERS.

TIC

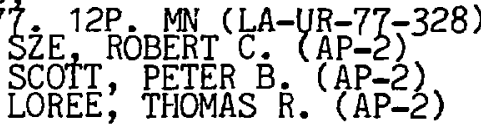

POLARIZATION POTENTIALS AND

ELEMENTARY EXCITATIONS IN LIQUID HELIUM -3 .

J. LOW TEMP. PHYS
V. P P $689-715$ ig78.
ALDRICH CHARLES H.
PINES, DAVID

$(M-6)$

52-54

POLARIZATION POTENTIALS AND ELEMENTARY EXCITATIONS IN LIQUID HELIUM -3 . BULL. AM. PHYS SOC.
V.23 P. $316-7$. 1978. ALDRICH, CHARLES H. (M-6)

$52-55$

POSITION SENSITIVE GAS

PROPORTIONAL CHAMBERS

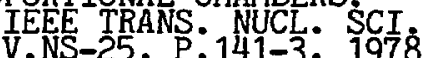

MORRIS, CHRISTOPHER L. (MP-10)

THIESSEN, HENRY A (MP-
HOFFMANN, G.W. (MP-10) 
$52-56$

PREDICTION OF MONTE CARLO ERRORS BY A

THEORY GENERALIZED TO TREAT TRACK

ENGTH ESTIMATORS.

NUCL. SCI. ENG.,

V. 65 , $\mathrm{P} .273-81: 21978$

AMSTER, THOMAS E. (TD-9)

52-57

PREIONIZATION OF PULSED GAS

LASERS BY RADIOACTIVE SOURCE. IEEE J QUANTUM ELECTRON. ,

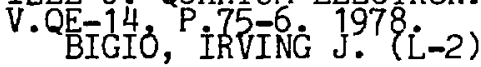

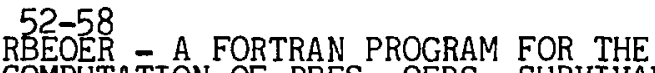

COMPUTATION OF RBES OERS SURVIVAL

RATIOS AND THE EFFECTS OF FRACTIONATION

USING THE THEORY OF DUAL RADIATION

ACTION.

LASL, 19P. (LA-7196-MS)
1978 19IDER; MARCO (MP-3). (MP-3)
DICELLO, JOHN F., JR. (MP-3)

RECENT DEVELOPMENTS IN THE SESAME

EQUATION - OF - STATE LIBRARY.

$$
\begin{aligned}
& \text { 1978. 19P. (LA-7130) } \\
& \text { BENNETT, BARD I., JR. (T-4) } \\
& \text { JOHNSON, JAMES D. }\left(\begin{array}{l}
\mathrm{T}-4 \\
\mathrm{~T}-4
\end{array}\right) \\
& \text { ROOD, GERALD T. (T-4) }
\end{aligned}
$$

52-60 RELATION IN THE ISING MODEL OF THE BEHAVIOR

J. APPL. PHYS, 1978.

BAKER GEORGE A., JR. (T-11)

MOUSSA, PIERRE

52-61

SHEAR-LAYER INSTABILITY IN

CYLINDRICAL IMPLOSIONS OF

ROTATING FLUIDS.

LASL,

78: 19P. (LA-7432-MS)

MJOLSNESS, RAYMOND C. $(\mathrm{T}-3)$

RUPPEL, HANS M. $(T-3)$

52-62

DERIVATION OF THE DIFFUSION

EQUATION FROM THE FOKKER-PLANCK

EQUATION USING PERTURBATION

METHODS

$$
\begin{aligned}
& \text { AM. J. PHYS. } \\
& \text { V. } 46 \text { P.392-3. } 1978 . \\
& \text { D ARR } \\
& \text { LARSEN, EDWARD W. (T-1) }
\end{aligned}
$$

52-63

SIMULTANEOUS MEASUREMENT IN FLUID N-D (SUB 2$)$ OF $P$ T $V$, T AND SOUND TIC 1977 . 16P. MN (LA-UR-77-739) ALSO PUBLISHED IN

THERMOPHYSICAL PROPERTIES.

1977. 7 TH SYMPOSIUM, GAITHERSBURG, MD. PROC. ' $P$.503-6. A. CEZAIRLIYAN,

$$
\begin{aligned}
& \text { LIEBENBERG DONALD H. }{ }^{(\mathrm{Q}-10)} \\
& \text { MILLS, ROBERT L. }(\mathrm{Q}-10)^{2} \\
& \text { BRONSON, JOHN C. }(\mathrm{Q}-10)
\end{aligned}
$$

52-64

STRUCTURE FACTOR OF A ONE - DIMENSIONAL SHOULDERED HARD - SPHERE FLUTD.

$$
\begin{aligned}
& \text { PHYS. LETT. } \\
& \text { V. } 5 \text { A P } 131-4.1978, \\
& \text { KINCAID JOHN } 19.11) \\
& \text { STELL, GEORGE }
\end{aligned}
$$

52-65

STUDIES OF LASER DRIVEN SHOCK WAVES IN ALUMINUM. PHYS. REV LETT. VEESER, IYNN R $(\dot{P}-3)$
SOLEM, JOHNDALE

52-66

SUPERFLUID DISPERSIONS. PHASE 1 PRELIMINAKY STUDY, OCTOBER 1976SEPTEMEER 1977.

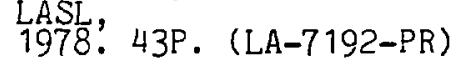

52-67

THEORETICAL ASSESSMENT OF RADIATION DAMIAGE EFFECTS IN A PROPOSED GAMMA RAY LASER SYSTEM.

$$
\begin{aligned}
& \text { LASL, 8OP. THESIS } \\
& 1978 \text { R } \\
& \text { RENSELAER POLYTECHNIC INST., } 1977 . \\
& \text { (LA-7099-T) } \\
& \text { SCHWENN, HAROLD R. (P-3) }
\end{aligned}
$$

52-68

THEORY OF AC AND DC ELECTRIC CONDUCTIVITY BY NONINTERACTING ELECTRONS IN CORRELATED ARPAYS OF FIXED SCATTERERS.

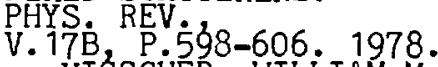
VISSCHER, WILIIAM M. (T-11)

52-69

THREE - DIMENSIONAL SHCCK - CHANGE RELATIONS FOR REACTIVE FLUIDS.

1978, 23P. (LA-7253) WACKERLE, JERFY D. $(W X-7)$ 
TIME DEPENDENCE OF THE COMPTON

CURRENT AND ENERGY DEPCSITION FROM

SCATTERED GAMMA RAYS.

$$
\begin{aligned}
& \text { 1978, 14P. (LA-7386-MS) } \\
& \text { MALIK, JOHN S. (J-DOT) } \\
& \text { CASHWELL, EDMOND D. (TD-6) } \\
& \text { SCHRANDT, ROBERT G. (TD-6) }
\end{aligned}
$$

\section{$52-71$}

VNAP - A COMPUTER PROGRAM FOR

COMPUTATION OF TWO-DIMENSIONAL,

TIME-DEPENDENT COMPRESSIBLE,
VISCOUS, INTERNAL FLOW.
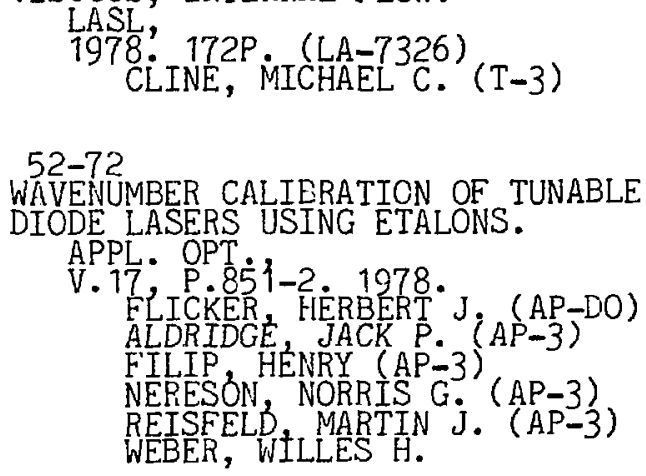


\section{3-1}

AMPLITUDE ANALYSIS OF PION-NUCLEUS

SCATTERING

P.Y 18

8C P. $2782-4.1978$.

GIBBS, WILLIAM $R$ ( $\mathrm{T}-5)$

GIBSON, BENJAMIN F. $(T-5)$

STEPHEISSON, GERARD J., JR. (T-5)

ANA-2

MCGRAW-HILL ENCYCLOPEDIA OF

SCIENCE AND TECHNOLOGY. 4TH ED. V. 1 , P. 406B-406D. MCGRAW-HILL, 1977.

(RE 503/M147E4)

STEIN, NELSON (P-9)

\section{$53-3$}

ANALYSIS OF ELASTIC SCATTERING

OF 0.8 GEV POLARIZED PROTONS FROM

TIN -116 AND TIN -124 .

PHYS. LETT

V.76B, $3.383-7.1978$.

HOFFMANN, G: W. (MP-10)

BILANPIED GARY S.

LIL JESTRAND, R.

RAY LANDON' (T-5) (MP-4)

THIESSEN, HENRY A. (MP-10)

HINTZ, N: M. OOTHOUDT,

BAUER, T.'S.

IGO, G.

PAULETTA, G.

WOUKUP, ${ }_{\text {WHITTEN, }}{ }^{\text {C. A. A JR. }}$

NANN, H?

SETH, KAMAL $K$.

MLASHAUSSER, $C$ DANIELS, DAVID $K$.

TINSLEY, J'

$53-4$

ANALYSIS OF 0.8 GEV POLARIZED,

PROTON ELASTIC SCATTERING FROM
LEAD -2OS, ZIRCONIUM -90, NICKEL -58

AND CARBON -12 .

PHYS. REV.

V. $18 \dot{C}, \quad$ P. $1756-60.1978$.

RAY LANDON (T-2)

HOFFMANN, G. W. $(M P-10)$

BLANPIED' GARY S. (MP-10)

COKER, WILLIAM R.

LILJESTRAND, R. (MP-10)
ANA-5 2 ING POWER IN PROTON NUCLEUS

ELASTIC SCATTERING AT 0.8 GEV.

PHYS. REV TETT. 1978

HOFFMANN, G: W. (MP-10)

BLANPIED' GARY S. (MP-10)

COKER, IILLIAM R.

HILJESTRAND,

OOTHOUDT, M. A.

BAUER, T': $S$.

PGOU GTTA, G.

SOUKUP, $J$.

WHITTER, $\dot{C}$. A., JR

MADLAND' DÄVID' G: $(T-2)$

RAY LANDON $(T-5)$

SPENCER, JAMES E. (MP-10)

THIESSEI, HENRY A. (MP-10)

IHANN, $\mathrm{H}$.

SETH, KAMAL K.

GLASHAUSSER, CC̈. DANIELS, DAVID K.

MINSLEY

VARGHESE, PHILIP

53-6

ANALYZING POWERS FOR D+D REACTIONS

FRON 13.39 TO $17.00 \mathrm{MEV}$.

LASL

1978, 10P. (LA-7545-MS)

EROWN, RONALD E. (P-7)

CORRELL, FRANC IS D. (P-7)

HAGLUND, R. F. JR. $(P-9)$

HARDEKOPF, FOEERT A. $(P-7)$

JARNIE, NELSON $(P-7)$

OHLSEN, GERALD G $(P-7)$

SCHMELZBACH, PIERRE

$53-7$

APPLICATION OF $\Lambda$ LIBRAFY OF

PROCESSED ENDF/B-IV FISSION - PRODUCT

AGGREGATE DECAY DATA IN THE

CALCULATION OF DECAY - EHERGY SPECTRA. LASL,

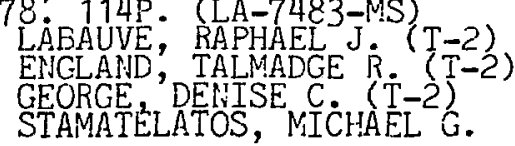

53-8

APFLIED NUCLEAR DATA RESEARCH

AND DEVELOPMENT, APRIL 1 - JUNE 30 , 1978.

1978: 45P. (LA-7482-PR)

BAXMAN' CLAIRE I. (T-2) COMP.
YOUNG, PHILLIP G. JR. (T-2) COMP. 
$53-9$

APPLIED NUCLEAR DATA RESEARCH AND

DEVELOPMENT, OCTOB

LASL,

1978:50P. (LA-7200-PR)

BAXMAN, CLAIRE I. (T-2) COMP. COMP.

$53-10$

APPLIED NUCLEAR DATA RESEARCH AND

DEVELOPMENT, JANUARY 1 - MARCH 31, 1978.

LASL, 28P. (LA-7301-PR)

BAXMAN, CLAIRE I. (T-2) COMP.

YOUNG, PHILLIP G., JR. (T-2) COMP.

$53-11$

BEAM PROF ILE MONITOR.

(LA-71 3 P2) AND PLATES.

KRAUSSE, GEORCE J. IIII $(M P-4)$

$53-12$

BISMUTH -210M (DEUTERON, PROTON)

BISMUTH - 211 REACTION AT $20 \mathrm{MEV}$.

PHYS. REV $1323-7.1978$.

HANSEN, OLE
COFFIN, J. P.
FLYNN, EDWARD R. (P-9)
STEIN, NELSON (P-9)
SHELINE, RAYMOND K.

$53-13$
CALCULATION OF NUCLEAR LEVEL

DENSITIES FOR IRON -56, CORALT -59,

COPPER -63; AND COPPER'-65.

NUCL. SCI. ENG.,

V. 66, P. 334-48:'1978.

FORD; GEORGE P! (CNC-11)

CALCULATION OF RADIATIVE SINGLE

CHARGE TRANSFER CROSS SECTIONS FOR

COLLISIONS OF MOLECULAR HELIUM ION 2+ WITH HELIUM AT LOW ENERGY.

PHYS. REV.

V. 18A, P. ióo4-8. 1978.

COHEN, JAMES S. $(\mathrm{T}-12)$

BARDSLEY, JAMES N. (T-4)

CALIBRATION OF A DETECTOR FOR PULSED

NEUTRON SOURCES.

LASL

1978: 8P. (LA-7022-MS)

VEESER, LYNN R $(P-3)(P-3)$

SHUNK, EDWARD R. $(P-3)$
$53-16$

-14 (TRITON, PROTON)

CAPBON - 16 REACTION AT $23 \mathrm{MEV}$.

V. 17C PE Pं $1919-23.1978$.

PETERSOl, $\dot{R}$.

FLYNA, EDWARD $\dot{R} .(P-7)$

53-17

CHARACTERISTICS, STABILITY, AND

SHORT-WAVELENGTH PHENOMENA IN

TWO-PHASE FLOW EQUATION SYSTEMS.

NUCL: SCI- ENG. ' 1978

RAMSHAW, JOHN D. (T-3)

TRAPP, JOHN A.

53-18

CHARGE AND DOUBLE CHARGE

EXCHANGE IN PION-NUCLEUS

SCATTERING.

HIGH ENERGY PHYSICS AND NUCLEAR

STRUCTURE. 1977 . INTERNATIONAL

CONFERENCE. TTH, ZURICH. PROC.,

PI 153-73. M. LOCHER ED. 1978.

(539.7/H638E 7TH) SPENCER, JAMES E. (MP-4)

CHA-19 ASYMMETRY IN HELIUM THREE HYDROGEN THREE AND THE NEUTRON PROTON MASS DIFFERENCE.

PHYS. REV $1456-60,1978$. GIBSON, BENJAMIN F. (T-5)

53-20

COMPARISON OF DISLOCATION PINNING BY NEUTRONS AND ELECTRONS.

BULL. AM. PHYS. SOC.,

V. 23 P. 289.1978.

GOLDSTONE, JOYCE A. (CMB-8)

PARKIN, DÓN M. (CMB-8)

SIMPSON, H. MITCHELL

COR-21 NUCL. PHYS.

V. 305A P. $461-84.1978$. JOHNSON, MIKKEL (MP-DO)

$53-22$

COULOMB ENERGIES IN S-SHELL NUCLEI

AND HYPERNUCLEI.

PHYS. REV.

GIBSON, BENJAMIN F. (T-5) 


\section{$53-24$}

CROSS SECTIONS FOR GAMMA - RAY NUCL. SCI. ENG. 1978. $\left.\begin{array}{l}\text { DRAKE DARRELL M: } \\ \text { ARTHUR, EDWARD D: } \\ \text { SILBERT, MYRON } \mathrm{G}-3 \\ \mathrm{P}-2 \\ \mathrm{P}-3\end{array}\right\}$

53-25

DEEP INELASTIC PION - INDUCED NUCLEAR REACTIONS IN THE ISOBAR MODEL

PHŸS. REV.

V. 17 GINOCCHIO, JOSEPH N. (T-5)

\section{$53-26$}

DETECTION OF THE NEW ISOTOPES, 12.3MINUTE MENDELEVIUM -258 .

\section{TIC}

HÖFFMÁ, MN (LA-UR-77-2901)

WILHELMY JERRY B. (CNC-11)

WEBER, JOSSEF (CNC-11)

DANIELS, WILLIAM R. (CNC-11)

HULET $E$. K.

LOANDRUM

WILD, J.'F.

53-27

DIFFERENCES BETWEEN LASL- AND ANL PROCESSED CROSS SECTIONS.

$$
\begin{aligned}
& \text { LASL, 161P. (LA-7197-MS) } \\
& 1978 \text { KIDMAN RUSSELL B. (T-2) } \\
& \text { MAC EARLANE ROBERT E. (T-2) } \\
& \text { BECKER, MARTIN }
\end{aligned}
$$

$53-28$

DIFFERENTIAL PRODUCTION CROSS

SECTIONS OF MULTIPLY CHARGED

FRAGMENTS IN 800 MEV PROTON INDUCED SPALLATION OF CARBON, ALUMINUM, AND NICKEL.

LASL

1978; 173P. THESIS, WASHINGTON

STATE UNTV, PULLMAN, 1978.

LUCKSTEAD, STEVEN C.

DYMAMICAL CONSTRAINTS ON THE

COUPLINGS OF THE GROUND STATE

MESONS. AN ESTTMATE OF THE PHI

YIELDS PI GAMMA DECAY.

PHYS. REV.

V. 17D P P . 1389-94. 1978.

RNED, JUNG S.

SLAUGHTER, MILTON D. (T-DO)

53-30

EFFECT OF PIOI! DISTORTION ON THE ASYMMETRY IN PRODUCTTON OF POSITIVENS ON LIGHT MUCLEI.

PHYS $\dot{17}, \stackrel{R E V}{P} \cdot \dot{8} 37-41.1978$. YOUNG, STEPHEN K. (T-5)
GIBBS, WLLLIAM R. (T-5)

53-31 OF SHORT-RANGE CORRELATIONS IN THE SECOND ORDER PION - CARBON OPTICAL POTENTIAL.

V.A3O2 P P $493-510.1978$ (T-5)
GARCILAZO, HUMBERTO

ELA-32 32 SCATTERING OF POSITIVE PIONS ON OXYGEN - 16 AT 40.0 AND 49.7 MEV. P. $17 \mathrm{C}$, REV

MALBROUCA 13901 . 1978.

DARDEN, C: W.

EDGE R. D.

MARKS, THOMAS

PREEDOM, BARRY $M$.

BURMAN ROBERT L. (MP-4)

MOINESTER, M. A. (MP-4)

REDh'TNE, ROBERT P. (MP-4)

BERTRAND $F$. E.

CLEARY, T. P.

GROSS, E. E.

GUDEMANA, C. A.

53-33 SCATTERING OF 162 MEV PIONS BY NUCLEI

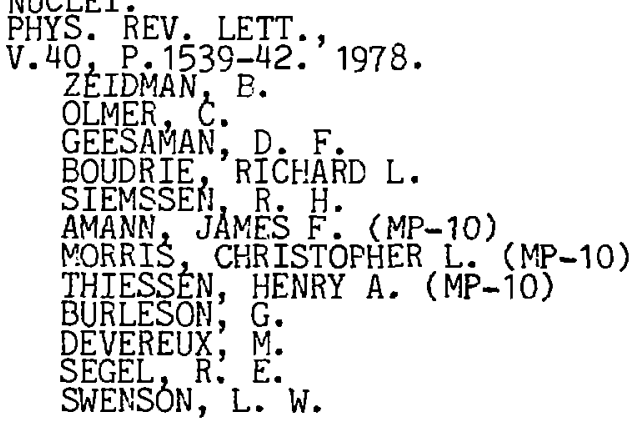


53-34

EMISSION OF SODIUM -24 FRAGMENTS IN THE INTERACTION OF GOLD -197 WITH INTERMEDIATE-ENERGY PIONS AND PROTONS.

PHYS: REV.

PORI $\dot{P}^{22} 31-40.1978$. DROPESKY, BRUCE J: ( CNC-11)

WILLIAMS; ROBERT $\dot{A}$. (CNC-11)

$53-35$

ENERGY LEVELS OF IRON -60

POPULATED BY THE IRON -58

(TRITON, PROTON) IRON -60 REACTION.

PHYS. REV.

V. 18C P. iỏ2-7. 1978.

MORMAN, ERIC $\mathrm{B}$.

MOSS, CALVIN E. (Q-2)

53-36

ENERGY RESOLUTION CONSIDERATIONS

IN LIQUID IONIZATION CHAMBERS.

TIC 77 . 5P. MN (LA-UR-77-2357)

ALSO PUBLISHED IN:

IEEE TRANS. WUCL. SCI, EDMISTON MICHAEL
GRUHN, CHARLES R.
E-10

ERT-37 THM THRESHOLD CHARGED PION PHOTOPRODUCTION AND RADIATIVE PION CAPTURE. II PI MINUUS PLUS DEUTERON GOES TO PHOTON PLUS TWO NEUTRONS.

(PHYS. REV. C 16, 327. 1977)

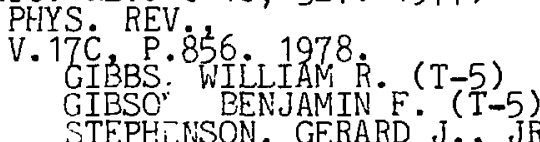

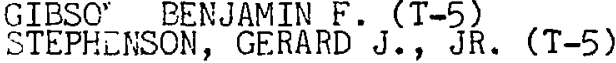

$53-38$

EVALUATION OF N PLUS PLUTONIUM -242

REACTIOIJS FROM $10 \mathrm{KEV}$ TO $20 \mathrm{MEV}$.

\section{LASL,}

1978

MADLAND (LA-7533-MS)

YOUNG, PHILLIP G., JR. (T-2)

53-39

EVALUATION OF THE LUTETIUM -175

(N, 2N)LUTETTUM -174 , LUTETIUM -175

(1N,2N)LUTETTUM $-174 M$, AND

LUTETIUM $-175(N, 3 N)$ LUUTETIUM -173

CROSS SECT
TO $20 \mathrm{MEV}$.

LASL,

1978. 31P. (LA-7236-MS)

YOUNG, PCILLIP G., JR. $(T-2)$
53-40

EVALUATION OF THE NEUTRON - INDUCED

REACTIONS FOR URANIUM -233 .

TRANS. AM. NUCL SOC.,

$8.721-2.1978$.

MADLAND, DENAD G. (T-2)

YOUNG, PHILLIP G., JR. (T-2)

EVENT MATRIX SYSTEM.

LASL,

1978. 3P. (LA-7143)

$53-42$

EXACT CALCULATION OF THE PENETRABILITY FOR A SIMPLE TWO - DIMENSIONAL HEAWY ION FUSION BARRIER.

PHYS. REV.

V. 17 KODAMA T11-7. 1978.

NAZARETH, R. A. M. S. MOLLER PETER $\{\mathrm{T}-\dot{9})$

$53-43$

EXTRACTING DELTAS FROM

THE DEUTERON.

V.A2 44 PHS. $293-304.1978$

COLDHABER, ALFRED S.' (T-5)

$53-44$

FABRICATION, CLADDING, AND HANDLING OF IRRADIATION TARGETS FOR ISOTOPE PRODUCTION AT LAMPE.

TRANS. AM. NUCL. SOC.

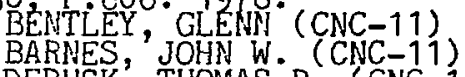

BARNES, JOHN W. (CNC-11)

DEBUSK THOMAS $P \dot{C}\left(\begin{array}{c}C N C-11 \\ C N C-11\end{array}\right)$

$53-45$

FAST NEUTRON CAPTURE CROSS SECTIONS OF THULIUM -169 , IRIDIUM -191 ,

IRIDIUM - 193 AND LUTETIUM -175 FOR 3 LESS THAN OR EQUAL TO E/SUB N/ LESS THAN OR EQUAL TO $2000 \mathrm{KEV}$. LASL

1978

MẢCKLIN, R. L T9-MS)

DRAKE DARRELL M. $\left(\begin{array}{l}P-3 \\ \text { MALANIFY, JOHN }\end{array}\right.$

FIS-46 AND FERMIUM.

$$
\begin{aligned}
& \text { TIC } \\
& 1978 \text {. 14P. MN (LA-UR-78-499) } \\
& \text { HOFFMAN, DARLEANE C. (CNC-11) }
\end{aligned}
$$


$53-47$

GENERALIZED REBALANCE. A COMMON FRAMEWORK FOR TRANSPORT

ACCELERATION METHODS.

NUCL. SCI ENG. ,

V.65, P.226-36.' 1978. MILLER, WARREN F.; JR. (T-1)

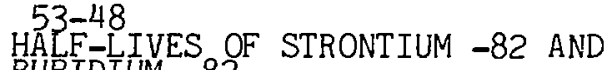

PHYS. REV

V. 18C P.2ं799-2800. 1978.

GRANT, PATRICK M, (CNC-11)

WHIPPLE, RICHARD E. (CNC-11)

DANIELS, REBECCA J. (CNC-11)

O"BRIEN, HAROLD A., JR. (CNC-11)

$53-49$

HYDRIDING OF TITANIUM CONES FOR A

SPUTTER ION SOURCE.

T978. 6P. MN (LA-UR-78-429)

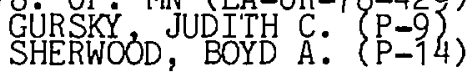

$53-50$

HYDROGEN -3 AND HELIUM -4 BINDING

ENERGIES IN THE "TRUNCATED

T-MATRIX" APPROXIMATION.

PHYS. REV. ${ }^{\circ}$ P. $1042-4.1978$.

GIBSON, BENJAMIN F: (T-5)

LEHMAN', DONALD R.

$53-51$

HYDROGEN AND HELIUM PRODUCTION CROSS

SECTIONS FOR ENDF/B-V

TRANS. AM NUCL SCC.,

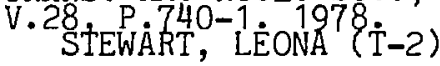

53-52 FORWARD AND BACKWARD SCATTERING OF

$\mathrm{N}-\mathrm{N}$ WITH SPIN.

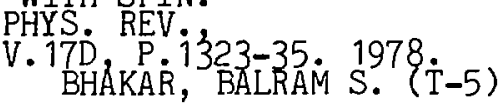

$53-53$

IMPORTANCE OF NONANALOG NUCLEON

CHARGE EXCHANGE TRANSITIONS IN PION KNOCKOUT REACTIONS.

PHYS. REV. $18 . \mathrm{C}, \mathrm{P} .2785-7.1978$.

SILBAR, RICHARD R. (T-5)

GINOCCHIO, JOSEPH N. (T-5)

STERNHEIM, MORTON M:
$53-54$

INTEGRAL DECAY - HEAT

MEASUREMENTS AND COMPARISONS TO

ENDF/B-IV AND V.

LASL

1978: 41P. (LA-7422)

ENGLAND, TALMADGE R. (T-2)

SCHENTER, R. E.

53-55

INTENSE NEUTRON SOURCE FACILITY -

OCTOBER 1 - DECEMBER 31, 1977.

LASL

1978: 55P. (LA-7156-PR)

BROWN, MARY C. $(\mathrm{P}-14)$ COMP.

53-56

INTENSE NEUTRON SOURCE FACILITY JANUARY 1 - MARCH 31, 1978.

LASL, 32P. (LA-7267-PR)

BROWN, MARY C. $(\mathrm{P}-14)$ COMP.

INTENSE NEUTROI SOURCE FACILITY, APRIL 1-JUNE 30, 1978.

1978. 14P. (LA-7473-PR)

BROWT, MARY C. $(P-14)$ COMP.

$53-58$

INTERACTION OF FAST NEUTRONS WITH

HELIUM -4, HELIUM -3 , AND

HYSROGEN -1. ADDITIONAL AND

IMPROVED DATA.

1978: 16P. (LA-7269-MS)
DROSG, MANFRED $(P-3)$

LITHIUM - 5 , TRITON REACTION ON MEDIUM MASS NUCLEI AND THE MASS AND SPECTRUM OF ZINC -61 .

$$
\begin{aligned}
& \text { PHYS. REV } \\
& \text { V. } 17 \dot{P} \text {. } 66-74.1978 . \\
& \text { WOODS, CLIFFORD W: (P-9) } \\
& \text { STEIN, NELSOI (P-9) } \\
& \text { SUNIER, JULES W. (P-9) }
\end{aligned}
$$

53-60

MEASUREMENT OF NP CHARGE EXCHANGE.

FOR NEUTRON ENERGIES 150-800 MEV.

PHYS. REV. LETT.
V. $41, P$. $1200-3.1978$,

BONNER, FILLY E. $(P-7)$

HOLLAS, C. L.

NEWSOM, C.

JAIN, MAIIAVIR (2-1) 
$53-61$

METHOD OF IMPROVING POSITION RESOLUTION OBTAINED FROM DELAY - LINE REACOUTS

$O F$ MNPC.

NUCL: INSTRUM METHODS,

MORRIS CHRISTOPHER L $L$ (MP-10)
HOFEMAN, G. W. (MP-10)

$53-62$

MODIFIED ONE-BODY NUCLEAR

DISSIPATION.

PHYS. REV

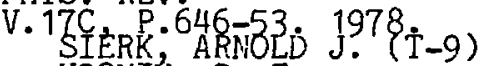

KOONIN S. S. $\stackrel{\text { E. }}{\text { NIX, JAME }}$. (T-9)

$53-63$

MODIFIED SURFACE ENERGY METHODS

FOR DERIVING HEAVY ION POTENTIALS.

HEAVY-ION ELASTIC SCATTERING.

1977 SYMPOSIUM UNIV. OF

ROCHESTER, WY. PAPERS, P.306-20.

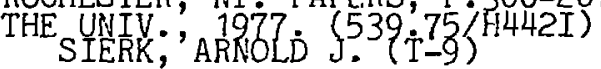

53-64

MULTIGROUP AND FEW - GROUP CROSS

SECTIONS FOR ENDF/B-IV FISSION

PRODUCTS. THE TOAFEW COLLAPSING

CODE AND DATA FILE OF 154 - GROUP

FISSION - PRODUCT CROSS SECTIONS.

LASL,

1978: 61P. (LA-7174-MS)

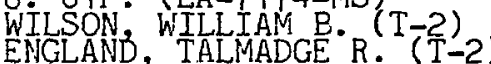

LABAUVE; RAPHAEL J. (T-2)

53-65

MUL TIPLE SCATTERING THEORY. I.

INTRODUCTION TO MULTIPLE

SCATTERING AND THE PION

NUCLEUS.

THEORETICAL METHODS IN

MEDIUM-ENERGY AND HEAVY ION

PHYSICS. K.W. MCVOY ED

GIBBS, WILLIAM R. $(\mathrm{T}-5)$

53-66

MULTIPLE SCATTERING THEORY. II.

FIXED NUCLEON APPROXIMATION.

THEORETICAL METHODS IN

PHYSICS, K.W. MCVOY ED.

P.510-31. PLENUM, 1978 (ं-5)
GIBBS, WILLIAM R. $(\mathrm{T}-5)$
MULTTIPOLE PAIRING STATES AND THE CORRESPONDENCE BETWEEN TWO - AND FOUR PARTICLE TRANSFER REACTIONS.

$$
\begin{aligned}
& \text { PHYS. LETT. } \\
& \text { V. T6B } P .47-50.1978 . \\
& \text { BETTS, RUSSELL R. } \\
& \text { STEIN, NIELSON (P-9) } \\
& \text { SUNIER, JULES W. (P-q) } \\
& \text { WOODS, CLIFFORD } w .(p-9)
\end{aligned}
$$

$53-68$

MUONIC ISOTOPE SHIFTS IN THE STABLE CALCIUM NUCLEI.

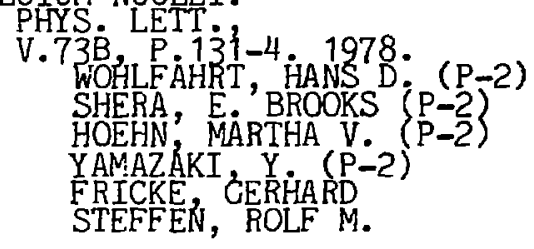

53-69

TE $\overline{\bar{R} B I U M}$ NUCLEI I I I DECAY OF $4.15-\mathrm{H}$ GADOLINIUM -149 .

PHYS. REV $1840-56.1978$

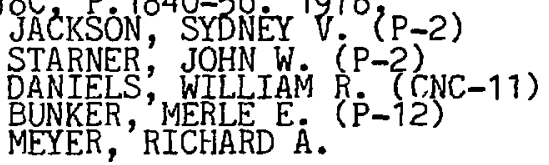

53-70

NEUTRON DEFORMATION PARAMETER

FROM COMPARATIVE STUDY OF

POSITIVE PION AND NEGATIVE

PION INELASTIC SCATTERING.

PHYS. REV. LETT 3978.

IVERSEN STEVEN (MP-10)

OBST, ANDREW (MP-10)

SET', KAMAL K. (MP-10)

THIESSEN, HENRY A (MP-10)

THIESSEN HERRY A $(M P-10)$
TANAKA, CHRISTOPHER L $(M P-10)$

SMITH, ELTON (MP-10)

AMANN, JAMES F. (MP-10)

BOUDRIE, RICHARD L.

BURLESON, G.

SWENSON, W.

VARGHESE, PHILIP

BOYER WITWAITE, WILFRED J.

COTTINGAME ${ }_{\text {MOORE, C. }}$. 
53-71

NEUTRON EMISSION FROM ACTINIDE

MUONIC ATOMS.

V. $18 \dot{C}$. P i 452-62. 1978.

WILCKE W. W. $\dot{M}$

SCHRODER, $\dot{W} . \dot{U}$.

PERRY, DENNIS $\dot{G}$. (MP-1)

53-72

NEUTRON MULTIPLICITY MEASUREMENTS

OF CALIFORNIUM AND FERMIUM

ISOTOPES.

$1977.21 P . M N$ (LA-UR-77-2916)

HOEFMAN DARLEANE C. (CNC-11)

FORD GEORGE P. (CNC-11)

BALAGNA, JOHN $P$. JR. (CNC-11)

VEESER, LYNN R.'(P-3)

$53-73$

NEUTRON SPECTRA CHARACTERISTICS

FOR THE INTENSE NEUTRON

SOURCE, INS.

TIC7'ं 17P. MN (LA-UR-77-1054)

EMIGH, $c$. ROBERT (P-14)

\section{$53-74$}

NEW UPPER LIMIT FOR MUON

DECAYING INTO AN ELECTRON

AND TWO GAMMAS.

PHYS. REV LETT

BOWMAN, J. DAVID (MP-4)

CHENC

LI LING-FONG

MATIS, H. S.

53-75 STNUCLEAR STATES AND THE

BETHE-SALPETER EQUATION.

PHYS. REV .

V. ${ }_{\text {TJON }}^{8 .}$, ${ }_{\text {JOHN }}^{2565-8}(\dot{T}-5)^{1978 .}$

NON-76 6 CAL CONTRIBUTIONS TO THE

RHO - OMEGA MIXING CHARGE

PHYS. REV. BREAKING FORCE.

V. $17 \dot{C}, 1752-62.1978$.

FRIAR, JAMES $\dot{L}$. (T- $-\dot{5})$

GIBSON, BENJAMIN $F$. $^{-5}(\mathrm{~T}-5)$

\section{3-77}

NUCLEAR DATA DEVELOPMENT AND SHIELD

DESIGN FOR NEUTRONS BELOW 60 MEV.

LASL: THESIS, TEXAS A \& $M$

UNIV: 1978 ' $115 \mathrm{P}$ (LA-7159-T)
WILSON, WILLIAM B. (T-2)
$53-78$
NUCLEAR

V. 40 ; REV $1605-8.97078$. DOOLEN, GARY D. (TD-2)

$53-79$

NUCLEAR STRUCTURE AND INELASTIC

ELECTRON SCATTERING REACTIONS.

THE SIGNIF ICANCE OF

HIGH-MOMENTUM-TRANSFER

DATA AND MESOH-EXCHANGE CURRENTS.

PHYS. REV. LETT.

V. 41 P P. 1453-7. 1978 .

HAITON, WICK C. (T-5)

53-80

NUCLEAR STRUCTURE OF PROMETHIUM -153

STUD IED WITH THE (TRITON, ALPHA) REACTION.

PHYS. REV BURKE D. G.

LOVHOTDEN
FLYNA
SUNIER, JUARD
JULES

53-81

OBSERVATION OF THE NEUTRON

PAIRING VIBRATIGN IN MERCURY - 206. PHYS, LETT $193-9.1078$ FLYNN EDWARD R. $\left\{\begin{array}{l}\mathrm{P}-7 \\ \mathrm{P}-7\end{array}\right)$ HANSON, DAVID L $\dot{P}_{-}(\mathrm{P}-7)$ ORBESEN, STUART D. (P-7) LIND, D. A.

53-82

ON CROSS SECTIONS FOR ELECTRON

IMPACT IONIZATION OF

MULTTCHARGED IONS.

IEEE TRANS. PLASMA SCI.

BARFIELD, $19.1978 \dot{\text { DAVID }}(\mathrm{T}-4)$

53-83

OSMIUM -188, $-190,-192$ (TRITON, PROTON) REACTION AT $15 \mathrm{MEV}$. PHYS. REV

V. $17 \dot{C}$, P. $581-7.1978$

ELYNN, EDWARD R. (P-9)
BURKE, D. G. 
PION -84

REMOYA INDUCED SINGLE - NUCLEON

PHYS. REV

V. $17 \dot{C}$ P. $237-36.1978$

MORRIS CHRISTOPHER L. (MP-10)

BOUDRIE RICHARD L.

KRAUSHAAR, J. J.

PETERSON, R. J.

RJSTINEN, R. A.

SMITH, GREGORY R.

BRA ITHWA ITE W. J.

MOORE, C. FRED

SMITH, L. E.

$53-85$

PION - NUCLEON FOFM FACTOR IN THE

CHEN-LOW THEORY.

PHYS. REV. $1747-58.1978$.

ERIST, DAVID J J (MP-DO)

53-86

PION DOUBLE - CHARGE EXCHAMGE ON

OXYGEN -16 AND OXYGEN - 18 .

PHYS. REV.

V. $17 \dot{C}$ P. $7774-86.1978$.

BURMAN, KOBERT L. (MP-4)

BAKER, MICHAEL P. $(Q-1)$

COOPER MARTIN D. $(M P-3)$

LEE, DAVID M. ( $M P-3)$

REDWINE, ROBERT P. (M:P-4)

SPENCER, JAMFS E. (MP-4)

MAFIKS, THOMAS

MALEROUGH, DONALD J.

PREEDOM, EARRY $M$.

HOLT, ROY J.

53-87

POSITIVE PION PLUS DEUTERON YIELDS PROT'ON PLUS PROTON REACTION AT 40, 50 , AND $60 \mathrm{MEV}$.

PHYS, REV.

$$
\begin{aligned}
& \text { V. 17̧ P P P } 1402-8.8978 . \\
& \text { DARDEN, } \mathrm{C} \text {. W. } \\
& \text { EDGE R. }{ }_{\text {MARKS }} \\
& \text { SALTMARSH, M. J. } \\
& \text { GABATHIULER, } K \text {. } \\
& \text { GROSS, E. E. } \\
& \text { LUDEMANH }{ }_{\dot{P}} \mathrm{C}_{\dot{Y}} A \text {. } \\
& \text { BLECIUR, } \dot{N}_{i} \\
& \text { GOTON, K. } \\
& \text { ALSTER, JONAS } \\
& \text { PERROUD, J } \mathrm{P} \text {. }
\end{aligned}
$$

$53-88$

PRESSURE GENERATION DUE TO A TEMPERATURE DISCONTINUITY AT LIQUTD - LIQUID PLANE INTERFACE. PHYS. FLUIDS

V.21, P. 334-46. 1978. COOPER BREWETI, PRETEICK J. $(\mathrm{Q}-7)$

5ROMPT NEUTRONS FROM NEUTRON - INDUCED FISSION OF NEPTUNIUM -237 .

PHYS, REV 17 C
VEESER, LYNN R.
R.

53-90

PROTON ELASTIC AND INELASTIC SCATTERING AT 0.8 GEV FROM CARBON

$-12,-13$ AND LEAD 208.

$$
\begin{aligned}
& \text { PHYS. REV } 1336-46.1978 \\
& \text { BLANPIED GARY S. (MP-10) } \\
& \text { COKER, WILLIAM R. } \\
& \text { HOFFMANN, G: W: }(M P-10) \\
& \text { RAY, LANDON (T-5) } \\
& \begin{array}{l}
\text { MADLAND, DAVID G. (T-2) } \\
\text { MORRIS, CHRISTOPHER L. (MP-10) } \\
\text { PRATT, JOHN C. (Q-2) }
\end{array} \\
& \begin{array}{l}
\text { SPENCER, JAMES E. }(M P-4) \\
\text { THIESSEN HENRY A. }(M P-10) \\
\text { KOZLOWSKI, THOMAS }(M P-1)
\end{array} \\
& \text { HINTE, N. M. } \\
& \text { OOTHOUDT, M. A. } \\
& \text { BAUER, T. S. } \\
& \text { ICO }, \mathrm{G} \text {. } \\
& \text { RIDGE, R. J. } \\
& \text { WHITTEN, C. A., JR. } \\
& \text { LANG, P. M. } \\
& \text { NANN, } H \text {. } \\
& \text { SETH, KAMAL K. }
\end{aligned}
$$

$53-91$

QUASIELASTIC CHARGE EXCHANGE IN NEUTRON DEUTERON YIELDS PROTON PLUS

NEUTRON PLUS NEUTRON AT $794 \mathrm{MEV}$.

PHYS. REV

V. $17 \dot{C}, P .6624-70.1978$.

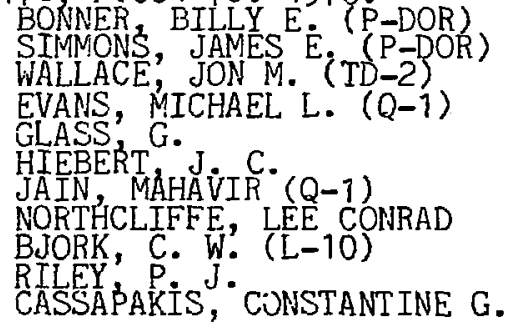


53-92

RAPID SPECTRAL AND DECAY - HEAT

CALCULATIONS USING PROCESSED ENDF/B

ISSION - PRODUCT DATA

TRANS. AM, NUCL. SOC.,

ENBAUVE, RAPHAEL $\dot{J}_{\dot{1}}(\mathrm{~T}-2)$

REIATIVE INTENSITIES OF GAMMA RAYS FKOM

HOLMIUM $166 \mathrm{M}$.

NUCL. INSTRUM. METHODS,

V. 150 P $361-3$ TH 1978.

RELATIVISTIC TWO - FLUID MODEL OF

NUCLEUS - NUCLEUS COLLISIONS.

PHYS. REV

V. 17C P $2080-96$ 1978
AMSDEN, ANTHONY A. (T-3)
GOLDHABER ALFRED S $(T-9)$
HARLOW FRANCIS H. $(\mathrm{T}-3)^{-9}$
NIX, JAMES R. (T-9)

53-95
REPLY TO "USE OF CHIRAL SYMMETRY IN PION CONDENSATION CALCULATIONS.

PHYS. REV

V. 18D, P. $1343-6.1978$.

CAMPBELL DA UID $K,(T-8)$
DASHEN ROGER F. $(T-8)$
MANASSAH, JAMAL T

53-96

SCATTERING OF POLARIZED

TRITONS BY BERYLLIUM -9 AND

CARBON -12 .

PHYS. REV

V.17C, P. 16-23. 1978

SCHMELZBACH: PIERRE

HARDEKOPF, ROBERT A. (P-Q)

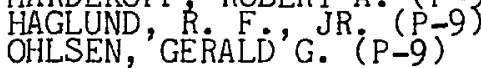

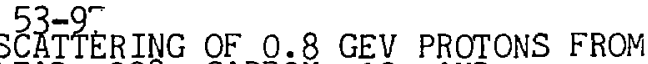

LEAD -208, CARBON -12, AND

CARBON -13 .

LASL,

1978: 131P. THESIS, UNIV. OF TEXAS

AT AUSTIN, 1978 . (LA- $7262-T)$
SHAPE TRANSITION BETWEEN $N=40$

AND 42 IN THE GALLIUM AND

GERMANILM ISOTOPES.

UNIV. PARIS SUD INST. DE

PHYSIQUE NUCLEATRE, ORSAY. 1977.

7P. AND PLATES. (IPNO-PHI-77-23)

VERCNES, M. N.

ROTEAPD, G.

ARDOUIN, $D$.

LEBRUN, C.
FLYNN, EDWARD R. $(P-9)$
HANSON, DAVID L.
ORBESEN, STUART D. (P-9)

$53-99$

SHIELDING FACTOR METHOD FOR

PRODUCING EFFECTIVE CROSS

SECTIONS. MINX/SPHINX AND THE

CCCC INTERFACE SYSTEM.

TIC 18 . 13P. MN (LA-UR-78-772) MÁC FARLANE, ROBERT E. (T-2) WEISBIN, $C$.' $R$. PAIK, N. C.

$53-100$

SPIN DETERMINATION OF RESONANCE

STRUCTURE. IN (URANIUM $-235+N$ )

BELOW 25 KEV.

PHYS. REV.

V. $18 \mathrm{C}, P$. $3328-48,1978$.
MOOKE, PICHAEL S.
MOSES, JOHN D. $(\dot{P}-3)$

KEYWORTH, GEORGE A. (P-3)

DABBS, J. W. T.

HILL, N.W.

STATES IN PLATINUM - 197 POPULATED BY THE (DEUTEROH, PROTOH) (DEUTEROH, TRITON) AND (NEUTRON, GAMMA)

REACTIONS.

PHYS. REV.

V. $17 \dot{C}, P$. $20861-70,1978$.

YAMAZAKI, $Y$. $(P-2)$

SHELINE, RAYMONE K.

STATUS OF THE ENDF/B SPECIAL APPLICATION FILES.

1977. 12P. MN (LA-UR-77-2140)

ALSO FUBLISHED IIV:

REACTOR DOSIMETRY
(OCT.) ASTM-E.URATOM SYMP. 2ND

(OCT.) ASTM-F.URATOM SYMP.

METHODS FOR FUELS, CLADDING,

AND STRUCTURAL MATERIALS.

P.843-53 (NUREG/CP -0OO 4 V.2)
STEWART, LEONA $(T-2)$ 
53-103

STRUCTURE OF GERMANIUM -78 FROM

THE GERMANTUM -76 (TRTTON, PROTON)

NANTES UNIV, FRANCE. INST. DE

PHYSIQUE. LAB. DE SPECTROSCOPIE

NUCLEA IRE AND LOS ALAMOS SCIENTIF IC

LABORATORY. RAPPORT INTERNE. 1978.

18P. (LSNN-78-02)

ALSO PUBLISHED IN:

PHYS. REV

V.18C P.1201-6. 1978.

ARDOUIN, D.

LEBRUN, C.

REMAUD, $B$;

FLYNN, EDWARD R. $(P-7)$

ORBESEN, STUART D. $(P-7)$

VERGNES, M. N.

ROTBARD, $G$

SY3-104

PRODUCTION BY 800 MEV PFOTONS

ON TARGETS WITH ATOMIC HUMBERS

FROM 27 TO 238.

PHYS. REV

V.18C, $P$, $1418-25.1078$.

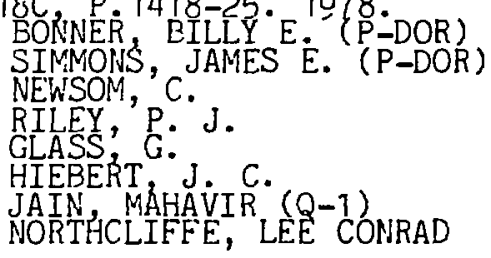

53-105

THIRESHOLD PION ELECTROPRODUCTION

ÂLD THE NUCLEAR RESPONSE SURFACE.

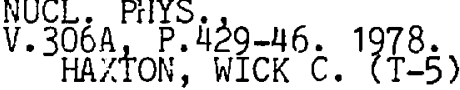

53-105

THRESHOLD PION PHOTOPRODUCTION

AND RADIATIVE PION CAPTURE. III.

HELIUM -3 GOES TO HYDROGEN -3 .

PHYS. FEV

V. 18. ${ }^{P}$. $T 761-3 .{ }^{1978}(T-5)$

GIBSOH, WENIAMIN $F$. (T $(T-5)$

STEPHENISON, GERARD J., JR. (T-5)

53-107

THRESHOLD PION PHOTOPRODUCTION IN

CARBON -12 AND THE $15.11 \mathrm{MEV}$

M1 FORM FACTOR.

PHYS. LETT
V.76̈ं
HAXTON, WIÉ
$53-108$

TRIDENT. A NEW TRIANGULAR MESH

DISCRETE ORDINATES CODE.

1978. 6P. MN (LA-UR-77-2511)

SEED THOMAS J. (T-1)

MILLER, WARREN F.', JR $(\mathrm{T})$ (T)

53-109

TRITIUM PRODUCTION IN A SPHERE OF (6)LID IRRADIATED BY 14 -MEV NEUTRONS.

LASL

978. 32P. (LA-7310)

HEMMENDINGER ARTHUR (P-3)

RAGAN, CHARLES E. (P...3)

ELLIS, ALEERT N: $(P-3)$

ANAYA, JOSE MANUEL $(P-3)$

$53-110$

UNCERTAINTIES IN NEUTRON DENSITIES

DETERMINED FROM ANALYSIS OF 0.8

GEV POLARIZED PROTON SCATTERING

FROM NUCLEI.

PHYS. REV

V. $18 \dot{C} \mathrm{C}^{2} \cdot \dot{2} 641-55.1278$;

RAY ROBERT L: (T-5)

COKER, WILLIAM R.

53-111

UNIFIED ABSOLUTE DIFFERENTIAL CROSS

SECTIONS FOR NEUTRON PRODUCTION BY T

HYDROGEN ISOTOPES FOR CHARGED PARTICL ENERGIES BETWEEN 6 AND 17 MEV.

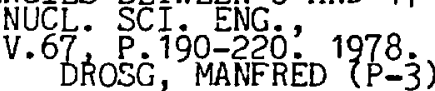

$53-112$

YETT MORE COMPLEXITY IN FISSION.

BARRIERS FOR NUCLEI WITH $\mathrm{N}=150-154$.

PHYS. REV LETT.

V. $40^{\circ}$ P $1010-3.3978$

BRITT, HAROLD C. (P-9)

GAVRON AVIGDOP I. ( $P-q)$

GOLDSTONE PHILIP D. (P-9)

SCHOENMACKERS, RUDI $(P-9)$

WEBER, JOE

WILHELIMY, JERRY B. (CNC-11)

53-113

4HE (D,D) 4HE SCATTERING FROM 12

TO 17 MEV.

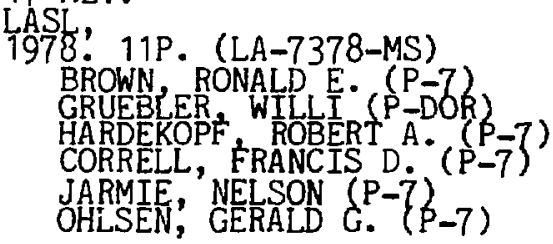


$54-1$

CALLORIMETRIC FISSION PRODUCT DECAY

HEAT MEASUREMENTS FOR

PLUTONIUM -239, URANIUM -233, AND

URANIUM -235 .

1978

YARNELL JOHN
BENDT, PHILIP J. $(P-8)$
$P-2$

$54-2$

CHANGES OE SYMMETRY IN WEAK INTERACTIONS

AT LARGE TRANSVERSE MOMENTA.

PHYS, REV $8379-83.1978$.

WEIEZER, SHALOM $\left(\begin{array}{l}\text { T-DO } \\ \text { WEINER, RICHARD }\end{array}\right.$

$54-3$

CHEMILUMINESCENT MEASUREMENT

SENSITIVITY OF A NON-UNIFORM

GAS.

J. QUANT - SPECTROSC RADIAT . TRANSFER,

V. 19. PAN 397-406. 1978 .

$54-4$

COHERENT STATES FOR GENERAL

POTENTIALS.

PHYS. REV. LETT.

V. 41 P. $207-10$. 1978.

NIETO NICHÄEL MART IN (T-8)

SIMMONS, LEONARD M. (T-DO)

\section{4-5}

DEPTH DEPENDENT AND TIME

AVERAGED DIFFUSION MODEL FOR

NITROGEN SATURATION/DESATURATION

PROCESSES.

INDIAN J TECHNOL

V. 15 P. 326-9.1977.

WIENKE, BRUCE R. (T-1)

\section{4-6}

DERIVATION OF THE VIRIAL

EXPANSION WITH APPLICATION TO

EUCLIDEAN QUANTUM FIELD THEORY.

J. MATH. PHYS. 19.1978

MENTKOFF RALPH $(\dot{T}-14)$
SHARP, DAVID H. $(\bar{T}-8)$

\section{4-7 7 OF CLASSICAL FIELDS IN MESON CORRELATIONS. \\ PHYS. REV. \\ V. 17D , P.3118-23. 1978.}

FOWLER, G. NAPAR (T-DO)
$54-8$

ESTIMATING POPULATION SIZES IN A MIXTURE OF TWO RADIOACTIVE POPULATIONS. LASL

978: 25P. (LA-7197)

QUEALR, WILLIAM A. $(T-7)$

EXA-2 CLASSICAL SOLUTIONS TO THE TWO

DINENSIONAL "SIGMA" MODEL - AN UNUSUAL APPLICATION OF INVERSE SCATTERING TECHNIQUES.

TIC

1976. 36P. MN (LA-UR-76-1924)

ALSO PUBLISHED IN:

ROCKY MT 37 J MATH 1978 .

CAMPBELL, DAVID K. (T-8)

54-10

FERMIONS AND BOSONS IN A UNIFIED

FRAMENORK. I. PHYSICAL FOUNDATIONS. PHYS. REV.

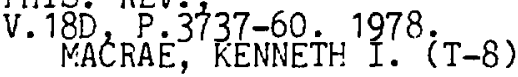

$54-11$

FERMIONS AND BOSONS IN A UNIFIED

FRAMEWORK. II. INTERACTING MODELS.

PHYS. REV $3261-76,1078$

MACRAEE, KENNETH I. (T-8)

$54-12$

INTERMULTIPLET MIXING OF THE

VECTOR MESONS IN A

NONPERTURBATIVE APPFOACH TO

BROKEN SU (4).

PHYS. KEV. LETT.

V. 39, P. 309-12: 1977 SLAUGHTER, MILTON D. (T-8)

54-13

IS THE FERMI THEORY OF WEAK

INTERACTIONS A YANG-MILLS THEORY IN DISGUISE?

PHYS. REV. LETT.

V. $40^{\circ}$ P. $1620-3.1978$ COOPER, FREDERICK"Y. (T-S) GURALNIK, G S.
SNYDERMAN, NEAL 
$54-14$

PATH INTEGRAL FORMULATION OF MEAN FIELD PERTURBATION THEORY.

ANN. PHYS.
V. 109, P $165-209 P .1977$.
BENDER, CARL M.
COOPER, FREDERICK M. (T-8)
GURALNIK, G. S.

$54-15$

STIMULATED EMISSION AND ABSORPTION

IN CLASSICAL SYSTEMS.

PHYS. REV.

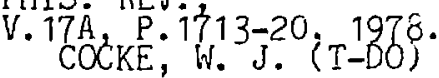

54-16

VARIATIONAL METHOD FOR BOSON

SCATTERING

$$
\begin{aligned}
& \text { PHYS. REV } \\
& \text { BOLSTERLI, MARK }(T-9)
\end{aligned}
$$

WILL' IHE AXION BE FOUND SOON?

PHYS. REV. LETT

V. 40 .

GOLDMAN, TERRENCE J $(T-8)$

HOFFMAN, CYRUS M. ( 
$55-1$

GENERAL - PURPOSE HEAT SOURCE

PROJECT, SPACE NUCLEAR SAFETY

PROGRAM' AND RADIOISOTOPIC

TERRESTRIAL SAFETY PROGRAM,

DECEMBER 1977.

LASL, 30P. (LA-7129-PR)
1978. BAKER, RICHARD D. (CMB-DO) COMP.

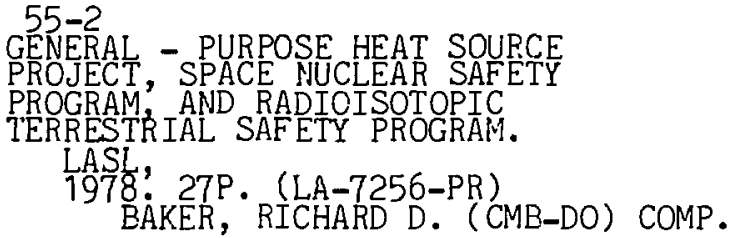

G5-3

DEVELOPMENT, PHASE 1 - DESIGN

REQUIREMENTS.

1978: 19P. (LA-7385-SR)

SNOW EDWARD CLARK (CMB-5)

ZOCHER, ROY W. (CMB-11)

$55-4$

GENERAL - PURPOSE HEAT SOURCE

PROJECT, SPACE NUCLEAR SAFETY PROGRAM AND RAD IOISOTOPIC TERRESTR IAL SAFETY

PROGRAM - JUNE 1978.

LASL, 35P. (LA-7416-PR)

BAKER, RICHARD D. (CMB-DO) COMP.

$55-5$

GENERAL - PURPOSE HEAT SOURCE

PROJECT, SPACE NUCLEAR SAFETY

PROGRAM!' AND RADIOISOTOPIC

TERRESTRIAL SAFETY HROGRAM,

JULY 1978 .

LASL

1978: 32P. (LA-7460-PR) BAKER, RICHARD D. (CME-DO) COMP.

GENERAL - PURPOSE HEAT SOURCE

PROJECT, SPACE NUCLEAR SAFETY

PROGRAM' AND RADIOISOTOPIC

TERRESTRIAL SAFETY PROGRAM,

AUGUST 1978.

$$
\begin{aligned}
& \text { LASL, } 51 \text { P. (LA-7519-PR) } \\
& \text { 1978 BAKER, RICHARD D. (CMB-DO) COMP. }
\end{aligned}
$$

GENE 5 -7

SPACE NUCLEAR SAFETY PROGRAM, AND

RADIOISOTOPIC TERRESTRIAL SAFETY

PROGRAM.

LASL, 33P. (LA-7381-PR)

BAKER, RICHARD D. (CME-DO) COMP.

$55-8$

GENERAL - PURPOSE HEAT SOURCE PROJECT, SPACE WUCLEAR SAEETY PROGRAM, AND RADIOISOTOPIC TERRESTRIAL SAFETY PROGRAM, JANUAKY 1978.

1978: 33P. (LA-7184-PR)

BAKER, RICHARD D. (CMB-DO) COMP.

$55-9$

GENERAL - PURPOSE IIEAT SOURCE PRCJECT, SPACE NUCLEAR SAFETY PROGRAM, AID RADIOISOTOPIC TERRESTRIAL SAFETY PROGRAM, FEBRUARY 1978.

LASL, 37P. (LA-7220-PR)
BAKER, RICHARD D. (C BAKER, RICHARD D. (CMB-DO) COMP.

$55-10$

GENERAL PURPOSE HEAT SOURCE

DEVELOPMENT. PHASE II - CONCEPTUAL DESIGNS.

LASL

1978. 20P (LA-7546-SR)

SHON, EDWAPD CLARK (CME-5)

ZCCLIER, ROY W.

HULEERT, L. E.

$55-11$

GENERAL PURPOSE HEAT SOURCE

PROJECT, SPACE NUCLEAR SAFETY

PROGRAM, AND RADIOISOTOPIC

TERRESTRIAL SAFETY PRCGRAM,

SEPTEMBER 1978.

LASL, 34P. (LA-7560-PR)

BAKER, RICHARD D. (CHE-DO)

$55-12$

GENERAL PURPOSE HEAT SOURCE PROJECT

SPACE NUCLEAR SAFETY PROGRAM, AND

RADIOISOTOPIC TERRESTRIAL SAFETY

PROGRAM, OCTOBER, 1978.

LASL, 35P. (LA-7601-PF)

BAKER, RICHARD D. (CMB-DO) COMP. 
$56-1$

ANALYTICAL METHODS FOR FISSIONABLE MATERIAL DETERMINATIONS IN THE

NUCLEAR FUEL CYCLE, OCTOBER 1, 1976-

SEPTEMEER 30, 1977.

LASL,

1978: 14P. (LA-7013-PR)

WATERBURY, GLENH R. (CMB-1) COMP.

CALIF 2 IFNIUM -252 BASED

NONDESTRUCTIVE ASSAY SYSTEM FOR

FISSILE MATERIAL.

NUCL. INSTRUM. METHODS,

V. $152, P .549-57.1978$.

MENLOVE HOWARD O; (Q-1)

$56-3$

COORDINATED SAFEGUARDS FOR MATERIALS

MANAGEMENT IN CHEMICAL SEPARATION,

CONVERSION, AND FUEL FABRICATION

FACILITIES

NUCL. MATER. MANAGEMENT,

V.7 ${ }_{\text {DAYEM, HASSAN } 126-3{ }^{8} \dot{Y}(Q-4)}$

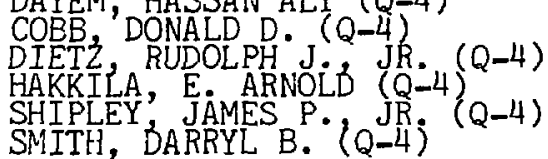

$56-4$

COORDINATED SAFEGUARDS FOR MATERIALS

MANAGEMENT IN A NITRATE-TO-OXIDE

CONVERSION FACILITY.

LASL, PAGED IN SECTIONS.
(LA-7011)

DAYEM, HASSAN ALY $(Q-4)$

COBB, DONALDD. (Q-4) $(Q-4)$

HAKKILA, E. ARNOĹ̉ $(Q-4)$

KERN, EDWARD A. (Q-4)

SHIPLEY JAMES P. JR; (Q-4)

SMITH DARRYL B. $(Q-4)$
BOWERSOX, DAVID F. (CMB-11)

56-5

CRITICAL REVIEW OF ANALYTICAL

TECHNIQUES FOR SAFEGUARDING

THE THORIUM-URANIUM FUEL CYCLE.

1978: 78P. (LA-7372)

HAKKILA, E. ARHOLD (Q-4)
DEC ISION ANALISIS FOR NUCLEAR SAFEGUARDS.

NUCLEAR SAFEGUARDS ANALYSIS.

NONDESTRUCTIVE AND ANALYTICAL

CHEMICAL TECHNIQUES. E. A. HAKKILA, ED P. $34-64$ ACS, 1978.

(621.48/N964SA)

SHIPLEY, JAMES P., JR. (Q-4)

$56-7$

DYMAC DEMONSTRATION PROGRAM. PHASE 1 EXPERIENCE.

1978 , 51P AND APPEND.

AUGUSTSON, RONALD H. (Q-3)

56-8

DYNAMIC MATERIALS CONTROL DEVELOPMENT

AND DEMONSTRATION PROGRAM.

NUCL. MATER MANAGEMENT,

V.7 PU 305-18. 1978 . H. (Q-3)
AUGUSTON, RONALD H.

EFFICIENT ANALYSIS OF DYNAMIC

MATERIALS ACCOUNTING DATA.

NUCL. MATER MANAGEMENT,

V.7 SHIPLEY, JAMES P., JR. (Q-4)

56-10

ASSAY MEASUREMENTS IN URANIUM

ENRICHMENT PLANTS.

LASL

1978: 51P. (LA-7212-MS)

WALTON, RODDY B. (Q-1)

$56-11$

HELIUM - 3 DETECTOR DESIGN FOR LOW LEVEL TRANSURANIC WASTE ASSAY.

TRANS. AM. NUCL SOC.",

HENDRICKS, JOHN $\dot{S}$. (TD-6)

CLOSE, DONALD A. ( $\mathrm{H}-\mathrm{T})$

$56-12$

INVENTORY AND VERIFICATION OF STORED NUCLEAR MATERIAL

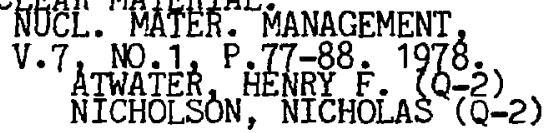


56-13

INVENTORY VERIFICATION IN LARGE

CRITICAL FACILITIES

NUCL MATER MANÄGEMENT,

V. $7, P .233-42.1978$. COBB, DONALD
SAPIR, JOSEPH L. $(Q-4)$
KERN, EDWARD A. (Q-4)

56-14

MORE ACCURATE THERMAL NEUTRON

COINCIDENCE COUNTING TECHNIQUE.

TIC
1978 BẢ $22 \mathrm{P} . M N$
BARON, NORTON (LA-UR-78-1444)

56-15

NEUTRON DETECTOR SUITCASE FOR THE

NUCLEAR EMERGENCY SEARCH TEAM.

1978: 6P. (LA-7108)

DOWDY, EDWARD J $(\mathrm{Q}-2)$

HENRY, CARL N. $(\mathrm{Q}-2)$

FRANCE, STEPHEN W. $(Q-2)$

56-16

NEUTRON INTERROGATOR ASSAY SYSTEM

PLANT WASTE CANISTERS AND SPENT

FUEL. PRELIMINARY DESCRIPTION AND

OPERATING PROCEDURES MANUAL.

LASI,

1978: 36P. (LA-7250-M)

MENLOVE, HOWARD $O$. (Q-1)

ECCLESTÓN, GEORGE W. $(Q-1)$

CLOSE, DONALD A $(H-1)(\mathrm{Q}-1)$

$56-17$

NON-DESTRUCTIVE ASSAY TECHNOLOGY AND AUTOMATED "REAL-TIME" MATERIALS CONTROL

NUCLEAR POWER AND ITS FUEL

CYCLE. 1977. INTERNATIONAL

CONF. SALZBURG, AUSTRTA. PROC. V.7. $397-614$. IAEA, 1977. 38/N964PP

KEEPIN, G. ROBERT (R-DO)

56-18

NONDESTRUCTIVE ASSAY METHODS FOR

IRRADIATED NUCLEAR FUELS.

LASL,

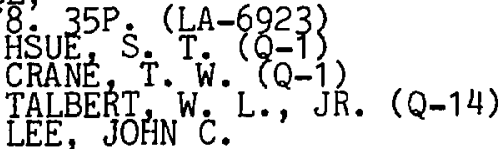

56-19

NONDESTRUCTIVE ASSAY OF HIGHLY

ENRICHED SPENT FUEL.

1978

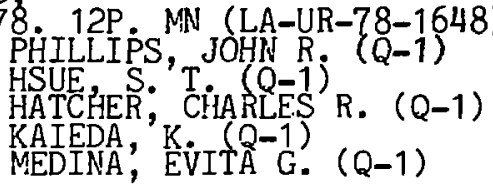

$56-20$

NUCLEAR SAFEGUARDS APPLICATIONS OF ENERGY-DISPERSIVE AESORPTION EDGE DENSITOMETRY.

AMERICAN CHEMICAL SOCIETY

NATTONAL MEETING. $197 \overline{8}$ (MAR.)

$175 \mathrm{TH}^{\circ}$ ANAHEIM, CA. ABSTRACTS OF

PAPERS, NUCL 21.

ALSO PUBLISHED IN:

NUCLEAR SAFEGUARDS ANALYSIS.

NONDESTRUCTIVE AND ANALYTICAL

CHEMICAL TECHNIQUES E.A. HAKKILA,

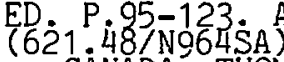
CANADA, THOMAS R. (Q-1)

LANCIER, $D$. $G \cdot\left\{\begin{array}{l}\dot{Q}-1 \\ Q-1\end{array}\right\}$

$56-21$

NUCLEAR SAFEGUARDS RESEARCH AND DEVELOPMENT PROGRAM STATUS REPORT MAY - AUGUST 1977.

LASL

1978: 86P. (LA-7030-PR)

SAPIR, JOSEPH L. (Q-DO) COMP.

$56-22$

NUCLEAR SAFEGUARDS, RESEARCH AND DEVELOPMENT. PROGRAM STATUS REPORT, LASL

1978: 88P. (LA-7211-PR)

SAPIR, JOSEPH L. (Q-DO) COMP.

$56-23$

ON-LINE ACCOUNTABILITY OF SPECIAL NUCLEAR MATERIAL (DYMAC).

CUBE SYMPOSIUM. 1978. LOS ALAMOS, NM. A1. LASL J.J. RUMINER COMP FÓRD, ROBERT $\dot{F}$. $(E-5)$

$56-24$

PRELIMINARY CONCEPTS - COORDINATED

SAFEGUARDS FOR MATERIALS MANAGEMENT

IN A THORIUM-URANIUN FUEL

REPROCESSING PLANT.

\section{LASL,}

1978'71P. (LA-7411-MS)

HAKKILA, E. ARNOLD (CMB-1)

BARNES,' JAMES $W$. (WX-4)

DAYEM, HASSAN ALY $(Q-4)$

DIETZ; RUDOLPH J., JR. (Q-4)

SHIPLEY, JAMES P.: JR. $\{Q-4\}$ 
$56-25$

RECONSTRUCTION OF AN ACCOUNT'S PAST.

NUCL. NATER MANAGEMENT,

WATERMAN, MICHAEL S. $\left\{\begin{array}{l}Q-12 \\ Q-12\end{array}\right.$

$56-26$

SAFETY ANALYSIS OF THE MAIN VAULT AT TA-4 1 SASL.

1978. 20P. (LA-6913)

EIRENKRAYZ, THEODORE E. ( $\mathrm{H}-3)$

DAHI DAVID A. (H-8)

POGNA, ROBERT (DIR-SEC)

$56-27$

STATISTICAL ASPECTS OF NUCLEAR SAFEGUARDS.

TIC
1977 IP. MN (LA-UR-7T-2420)
TIETEN, GARY L. $(Q-12)$

$56-28$

TRUCTURE OF SAFEGUARDS SYSTEMS.

1978: 17P. (LA-7337-MS)

SHIPLEY, JAMESP., JR. (Q-4)

$56-29$

SUMMARY OF INDICATORS OF NTH COUNTRY WEAPON DEVELOPMENT PROGRAMS.

LASL, 1978. (LA-6904-MS)

DÖUGHERTY, JOHN E. (TD-7)

$56-30$

TECHNICAL IMPLEMENTATION OF

SAFEGUARDS IN THE NUCLEAR FUEL CYCLE. TRAHS. AM NUCL SOC. ,

KEEPIN, G. ROBERT (Q-DO)

$56-31$

TITLES AND ABSTRACTS OF RECENT

SAFEGUARDS R\&D PUBLICATIONS AND

REPORTS.

NUCL: NATER. MANACEMENT,
V.6 NO.4 P. $31-2.1978$.
SAFECUARDS GROUPS
$56-32$

TRANSURANIC WASTE ASSAY

INSTRUMENTATION - NEW DEVELOPMENTS

SCIENTIFIC LABORATORY

NUCL. MATER. MANAGEMENT,

$V .7$ P $503-14.1978$.

CLOSE DONALD A. $(\mathrm{H}-1)$

UMBARGER ${ }^{C}$. JOHN $(\mathrm{H}-1)$

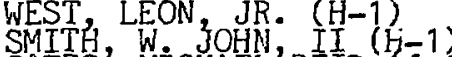

CATES, MICHAEL'REID $(\mathrm{J}-14)$

NOEL, BRUCE W. $(\mathrm{j}-8)$

FRANKS, L, A.

TRUNDLE, A. S. 
57. SOLAR THERMAL

\section{$57-1$}

AUGMENTED SOLAR ENERGY COLLECTTON USING VARIOUS PLANAR RETLECTIVE SURFACES. THEORETTCAL CALCULATIONS AND EXPERIMENTAL RESULTS. 197 1978:36P. (LA-07041) GRIMMER, DERRICK P. (Q-11) ZINN, KENNETH C. WOOD; B. E.

\section{$57-2$}

ELOOD LAMP SHAPED CONCENTRATING COLLECTORS FOR SOLAR THERMAL OR TOTAL SOLAR APPLICATIONS.

CONCENTRATING SOLAR COLLECTORS. 1977$. SEPT $)$ : ERDA CONE SÓNTRATING COLLECTORS. P $5-121-8$. THE CONF., GRIMMER, DERRICK P. (Q-11)

\section{$57-3$}

POSSIBLE SELECTIVE SOLAR

PHOTOTHERMAL ABSORBER. NICKEL DENDRITES FORMED ON

ALUMINUM SURFACES BY THE

CVD OF NICKEL CARBONYL.

V. $15, P .59-64.1978$.

GRIMMER, DERRICK P. (2-24)

HERR, KENNETH C.

MC CREARY, WILLIAM J. (CMB-6)

SOLAR

HYDROGEN FROM WATER.

1978 . 16P. MN (LA-UR-78-1052)

COX KENNETH E. (CMB-3)

BOWMAN, MELVIN ${ }^{\circ}$. ( $(C M B-D O)$

$57-5$

APPLICATIONS.

1978. 10P. MN (LA-UR-78-929)

COLLIER, ROBERT K. $(Q-11)$

$57-6$

SOLAR COLLECTORS, PART II. RECENT DEVELOPMENTS AND FUTURE PERFORMANCE DATA AND ECONOMIC ANALYSIS.

TIC. $26 \mathrm{CP}$. MN (LA-UR-78-1297)
TRE-7MENT OF MOLYBDENITE ORE USING A 2 KM SOLAR FURNACE.

1973 S. 17P. MA (LA-UR-78-1051) COUTURES, JEAN-PIERRE 
58-1

DENSITY GRADIENT DRIFT INSTABILITIES.

OBLIQUE PROPAGATION AT ZERC BETA.

$$
\begin{aligned}
& \text { PHY S. FLUTDS, } \\
& \text { V. } 1 \text { P P 1181-7 1978, } \\
& \text { GARY S. PETER (CTR-6) } \\
& \text { SANDERSON, JEFFREY J. (CTR-6) }
\end{aligned}
$$

\section{8-2}

EFFECTS OF AN AXIAL HEAT FLUX

LIMIT IN LINEAR THETA PINCHES.

PHYS. FLUTDS 1978.

GARY; S. PETER (CTR-6)

DE SILVA, ALAN W.

$58-3$

EMISSION COEFFICIENT FOR A SINGLY

IONIZED URANIUM PLASMA. EXPERIMENTAL

AND THEORETICAL TREATMENT.

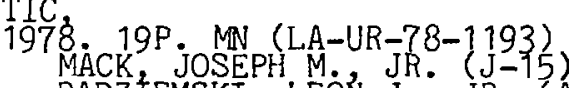

$$
\begin{aligned}
& \text { RADZIEMSKI, LEÓN J., JR. (AP-4) }
\end{aligned}
$$

$58-4$

ENERGY ANISOTROPY INSTABILITIES IN

HIGH - BETA PLASMAS. A COMPARISON

OF VARIOUS KINETIC AND FLUID

DESCRIPTIONS

PHYS. FLUIDS,

V.21:P.1790-1802. 1978.
CAYTON, THOMAS (CTR-6)

$58-5$

FINITE LARMOR RADIUS EQUATIONS

IN AN ARBITRARY NEAR - THETA

PINCH GEOMETRY.

PHYS. FLUIDS,

V.21 P. 1218-26, 1978.

FREIDBERG, JEFFREY P. (CTR-6)

58-6

INFRARED HETERODYNE INTERFEROMETER

FOR MULTICHORD PLASMA DENSITY

MEASURENENTS.

HIGH-TEMPERATURE PLASMA DIAGNOSTICS.

1978. 2ND TOPICAL CONF SANTA FE,

MM. ABSTRACTS P. 59 ' ( $L A-7160-C)$
JACOBSON, ABRAM'R. (CTR-2)
MI-7 7 WAVE ABSORPTION STUDIES

NEAR THE PLASMA FREQUENCY.

$$
\begin{aligned}
& \text { TIC } 1978 \text { 5P. MN (LA-UR-78-51) } \\
& \text { WRIGHT, BRADFORD L. (CTR-1) } \\
& \text { BANTON, MARTIN E. } \\
& \text { DRETCER, HARRY (CTR-DO) } \\
& \text { INGRAHAM, JOHN C. (CTR-1) }
\end{aligned}
$$

$58-8$

MULTIDIMENSIONAL THEORY OF THE

INHOMOGENEOUS BEAM - PLASMA

INSTABILITY

PHYS. FLUIDS

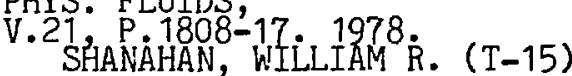

58-9

PARTICLE SIMULATION STUDIES OF THE LOWER HYBRID DRIFT INSTABILITY.

PHYS. FLUTDS,
V. 21 P $1017-25,1078$.
WINSKE, DAN (CTR-6;
LIEWER, PAULETTE C.

$58-10$

RELATIVISTIC DERIVATION OF - LASER

INDUCED PLASMA PROFILES.

PHYS. FLUIDS

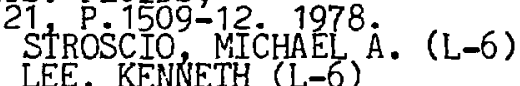

LEE, KENNETH (L-6)
LINDMAN, ERICK L. (L-6)

$58-11$

ROTATIONAL INSTABILITIES IN A

THETA PINCH.

PHYS. FLUIDS,

V.21: P.1207-17. 1978.

FREIDBERG, JEFFREY P. (CTR-6)

PEARLSTEIN, L. D.

SIMPLE MEASUREMENT OF LINE DENSITY.

LASL, 3P. (LA-7127-MS)

ARMSTRONG WILLIAM T. (CTR-3) 
59. TRANSPORTATION OF PROPERTY AND NUCLEAR MATERIALS

CÁRGO RESPONSE TO RAILCAR IMPACT AND TIEDOWN LOAD ANALYSIS.

1978, 54P. (LA-7469-MS)

BARTHOLOMEW, ROEERT J. (WX-8)

$59-2$

LINEAR AND NONLINEAR SYMMETRICALLY

LOADED SHELLS OF REVOLUTION

APPROXIMATED WITH THE FINITE

ELEMENT METHOD.

1978: 57P (LA-7538-MS)

COOK, WILLIAM A. (WX-8)

REUIEW OF THE BASES FOR REGULATIONS

GOVERNING THE TRANSPORT OF FISSILE

AND OTHER RADIOACTIVE MATERIAL.

T978. 5P. MN (LA-UR-78-1296)

SMITH' DAVID R. (Q-14)
THOMAS, JOE T.

SAFF-4 TEST OE AN IMPROVED

MULT THUNDRED WATT FSA. LAUNCH

ABORT SOLID PROPELLANT FIRE.

1978: 22P (LA-7314-MS)

SEABOURN, C. MELVIN (CMB-5) 


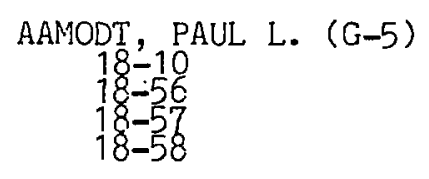

$\underset{21-2}{A A M O D T}, R$ LEE $(G-3)$

ABEELE, WILLY V. (H-8)

ABERIATTEY, ROBERT M. (CMB-1) $5-30$

ACKERHALT, JAY R. (T-12)

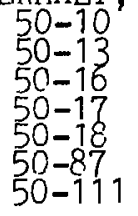

ADAMS, THOMAS F. $(J-15)$

ADAMS, WARREN H. (H-7) $18-68$
$18-69$

AGNEW, HAROLD M. (DIR) $28-2$
$28-6$

$\underset{18-31}{A H L Q U T S T}$ A. JOHN $(H-8)$

AHRENKIEL, RICHARD K. (L-9) $32-41$
$32-70$
$32-79$

AIKEN CARLOS L. V.

$\underset{\substack{5-1 \\ 5-4}}{\text { ALARID }}$, J. E. (CMB-1)

$\underset{50-7}{A L B E R S,}$ ROBERT C. $(\mathrm{T}-11)$
$50-8$

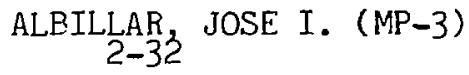

ALCOUFFE, RAYMOND E. (T-1)

$26-24$
$26-25$
$36-1$

ALDRICH, CHARLES H. $(M-6)$

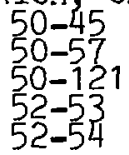

$\underset{52-72}{A L D R I D G E}$ JACK P. (AP-3)

$\underset{50-5 ;}{\operatorname{ALDRIDGE}}$, JACK P. (AP-4)

ALEI, MOHAMMED, JR. (CNC-4)

$5-19$
$5-26$
$50-79$

ALLEN ${ }_{41-66}^{\text {RICHARD C. (C-DIV) }}$

ALLRED, JOHN C. (MP-3)

ALSTER, JONAS
53-87

AMANN, JAMES F. (MP-10)

$53-73$

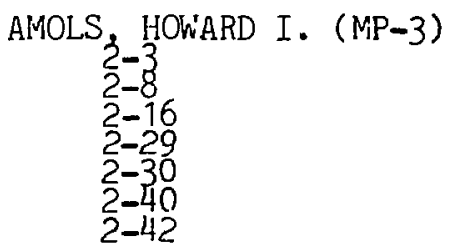

AMSDEN, ANTHONY A. (T-3)

$40-12$

$41-58$
$53-94$ 
AMSTER, $\underset{52-56}{\text { HARVEY J. }}$

ANAYA, JOSE MANUEL $(P-3)$

ANDERSON, CHARLES A. (Q-13)

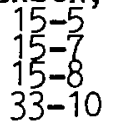

$\underset{2-71}{\operatorname{ANDERSON}} \underset{2}{\text { ERNEST C. (H-DO) }}$

$\underset{34-3}{\text { ANDERSON, GRAYDON K. }}(A P-3)$

$\underset{39-24}{\text { ANDERSON, JAMES L. (CMB-3) }}$

$\underset{34-19}{\text { ANDERSON, }}$ ROBERT G. $(A P-3)$

ANDRAE, RICHARD W. (WX-8)

$16-2$
$16-25$
$41-24$

APPERSON, COURTNEY E., JR. (Q-13) $25-7$

APT, KENNETH E. (CNC-DO)

$18-26$
$18-34$

ARCHULETA, JACOBO R. $(G-4)$

$23-1$
$23-2$
$23-4$

ARCHULETA, JOHN A. (H-8)

$18-22$
$18-37$
$51-47$

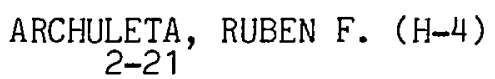

ARDOUIN, D.
$53-98$
$53-103$

ARMSTRONG, WILLIAM T. (CTR-11) 19-9

ARMSTRONG, WILLIAM T. (CTR-3)
$19-10$
$39-18$
$58-12$

ARTHUR, EDWARD D. (T-2)
$53-24$
$53-39$

ASERIDGE, JOHN R. (P-4)

$51-10$

51-13

$51-145$

$51-26$
$51-29$

$51-33$

$51-38$

$5:-54$

ASPREY, LARNED E. (CNC-4)
$34-9$

ASPREY, MARGARET W. (Q-7)

$\underset{56-12}{\text { ATWATER }}$ [IENRY F. (Q-2)

AUGLSTSON, RONALD $H .(Q-3)$
$56-7$
$56-8$

AVITABILE, JOHN N., JR. $(T-10)$
$50-14$

BABCOCK, RICHARD V.
$32-103$

BACA $\underset{41-22}{ }$ G.

BACKSEN, MICHAEL H. (E-5)

BACKUS, C. E.

BAIN, ELVIRA $(\mathrm{H}-10)$

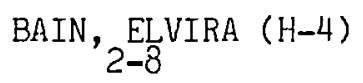

BAKER, DANIEL N. $(\mathrm{P}-4)$ 


$$
\begin{gathered}
51-20 \\
51=20 \\
5=22
\end{gathered}
$$

BAKER GEORGE A., JR. $(T-11)$
$41-30$
$52-31$
$52-60$

$\underset{53-86}{\text { BAKER }}$ MICHAEL P. (Q-1)

BAKER ${ }_{55-11}^{2}$ RICHARD D. (CMB-DO)

EAKER, RICHARD D. (CMB-DO) COMP.

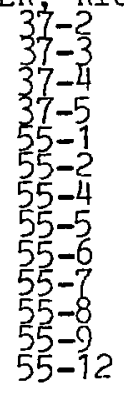

BALAGNA, JOHN P., JR. (CNC-11)

$18-34$
$44-6$

$53-72$

BALCOMB, J. DOUGLAS (Q-DO)

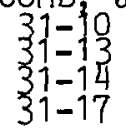

BALDWIN ${ }_{16}-13$ HOWARD A.

$\underset{34-2}{\text { BALOG, GEORGE }(A P-2)}$

BAME, SAMUEL J., JR. (P-4)

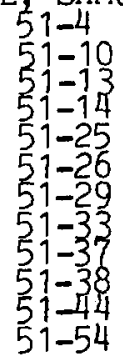

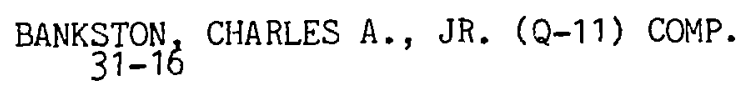

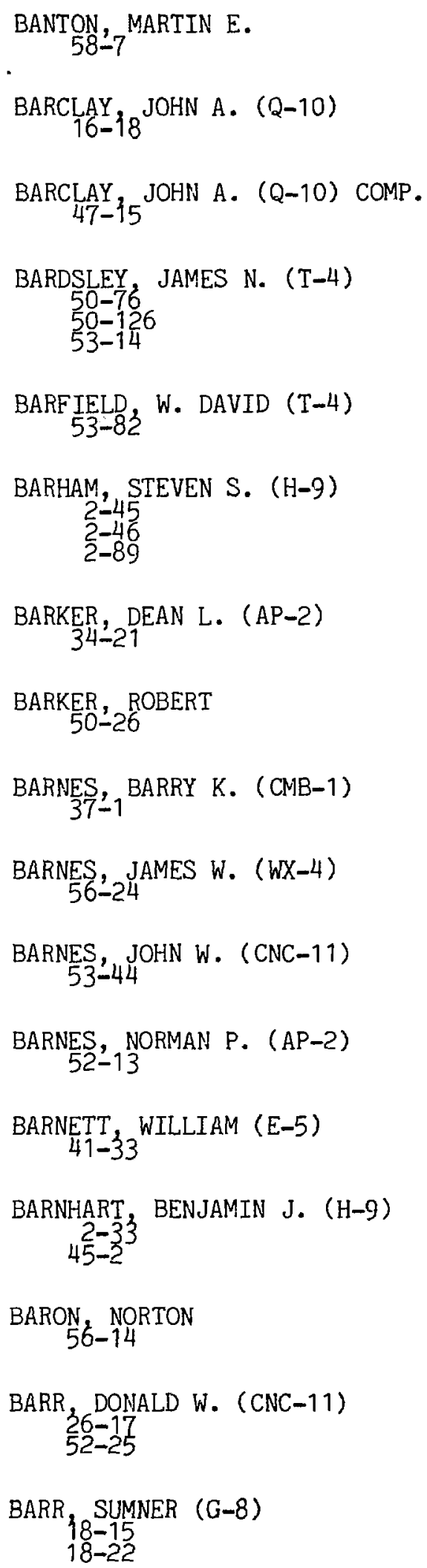




\section{$18-37$
$51-47$}

BARRACLOUGH, BRUCE (CMB-1)

BARTHOLOMEW, ROBERT J. (WX-3) $18-35$
$59-1$

BARTLETT
$2-79$

$\underset{52-50}{\text { BARTLETT ROGER J. }(Q-10)}$

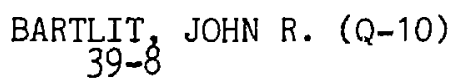

BARTS, EDWARD W. (Q-7)

BARTSCH, R. R. (CTR-3) $20=11$
$20-12$

BATTAT, MORRIS E. (T-1) 53-73

BAUER, T. S.

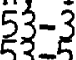

53-90

BAXMAN, CLAIRE I. (T-2) COMP. $53-8$
$53-9$
$53-10$

BAYTOS, JOHN F. $(W X-3)$

BEATTIE 3 WILLARD H. (AP-4)
$34-16$
$34-18$
$50-97$

BECKER, MARTIN $26-3$
$53-27$

$\underset{\substack{\text { BECKMAN } \\ 41-30 \\ 41-65}}{\text { RICHARD J. (Q-12) }}$

BECKMAN, RICHARD J. (S-DO)
BEGLEY, RICHARD F. (AP-4)

BEHRENS, ROBERT G. (CME-3)
$25-6$
$40-25$

BELIAN, RICHARD D. (P-4)

$$
\begin{aligned}
& 51-20 \\
& 51-21 \\
& 51-22
\end{aligned}
$$

${ }_{38-7}^{\text {BELL, }} \underset{3 \text { RLES }}{\text { R. }}(\mathrm{Q}-7)$

BELL, $\underset{5-25}{\text { GEORGE I. (T-DO) }}$

BELL, GEORGE I. (T-10)

BEMENT, THOMAS R. $(Q-12)$
$\substack{44-1 \\ 44-3}$

BEMENT, THOMAS R. $(S-1)$

BEN-DAVID, SHAUL
$31-9$

BENDER, CARL $M$.

BENDT, PHILIP J. (P-2)
$16-7$
$50-40$
$54-1$

BENJAMIN
$32-63$

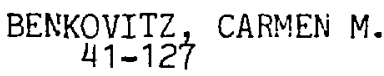

BENNETT $252-59$ BARD I., JR. (T-4)

BENNETT, JOEL G. (Q-13)

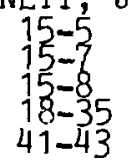

BENTLEY, GLENN (CNC-11) 


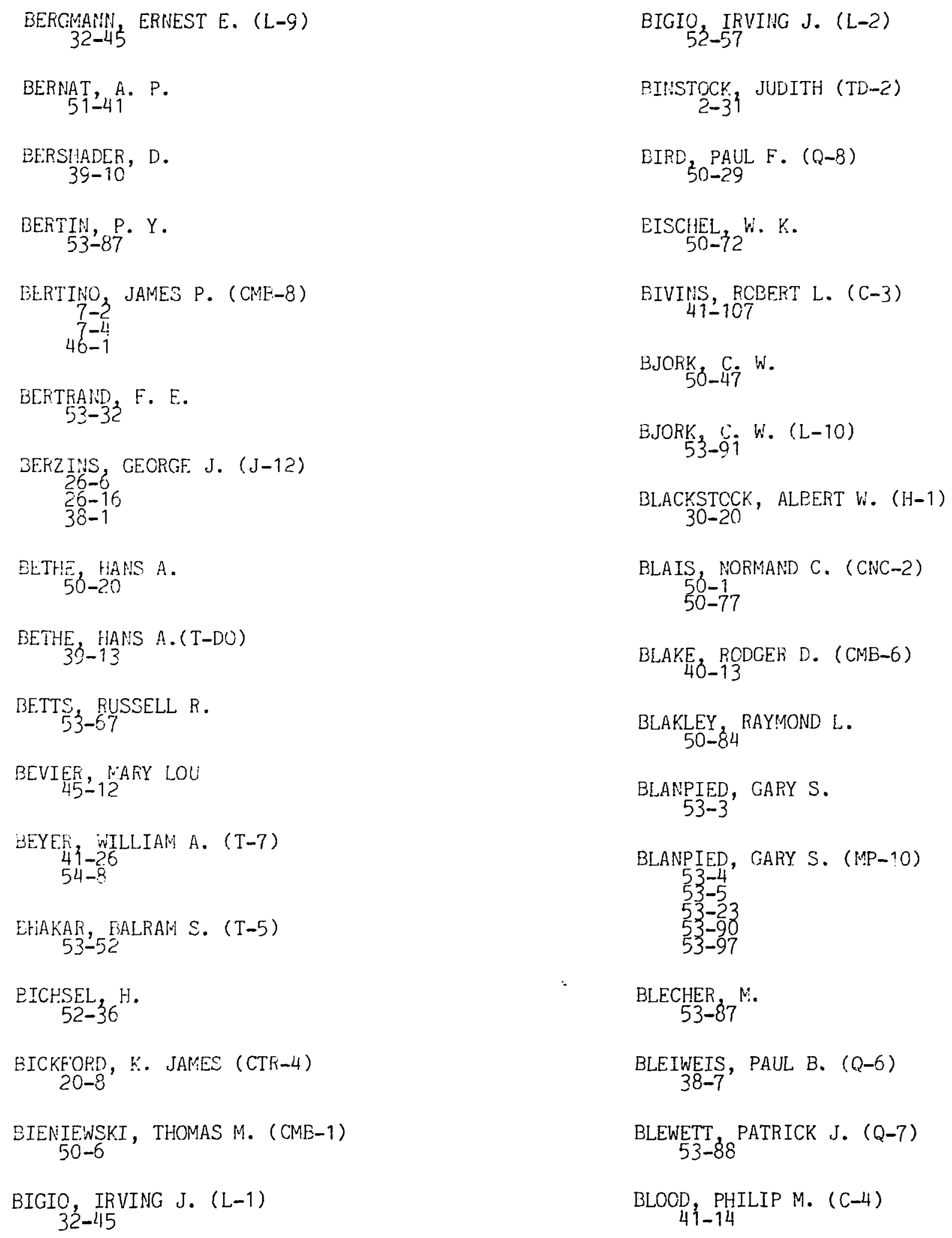




$$
\begin{aligned}
& \text { BOBEETT, RONALD E. (E-4) } \\
& \text { BOBEETT, RONALD E. (E-4) ED. }
\end{aligned}
$$

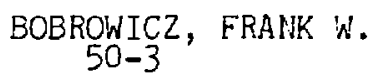

BOENIG, HEINRICH J. (Q-10)

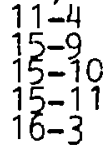

BOHACHEVSKY, I. O. $(L-5)$
$20-14$
$32-18$
$32-44$
$32-83$

BOHACHEVSKY, I. O. (L-5) TRANSLATOR $32-46$

BOHL, WILLIAM R. (Q-7)

$38-7$

BOICOURT, GRENFELL P. (CTR-11) $16-19$
$20-3$
$20-8$

BOLEY D. LUCIUS

$\underset{53-84}{\text { BOLGER, JOSEPH E. }}$

BOLIVAR, STEPHEN L. (G-5)

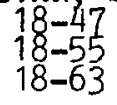

$\underset{26-1}{\operatorname{BOLSTAD}}$ JOHN W. $(Q-6)$

BOLSTERLI, MARK $(T-9)$

BONGIANNI, $\frac{\text { T. L. }}{32-10}$

BONNER, BILLY E. (P-DOR)

50-47

$53-91$
$53-104$
BONIER, ${ }_{53}-60$ ILLY E. (P-7)

BOOTH, LAWRENCE A. (L-5)

20-14

$32-7$
$32-39$
$32-44$

$32-83$
$32-92$

BOOTH, THONAS E. (TD-G)

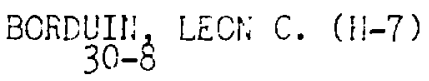

BORIE $50-53$

BORING, A. MICHAEL (CNC-3)

BORING, 4. NICHAEL $(T-12)$

BOSLER, GERALE E. $(T-1)$

$20-21$

53-108

BOSTICK, KENLETH V. $(H-3)$

BOLDREAU, JAY E. $(Q-7)$
$38-7$

BOUDRIE, FICHARE L.

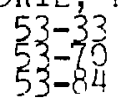

BOWERS, NCRMAN
$16-27$

BOWERSOX, DAVIL F. $(C N E-11)$
$26-4$
$56-4$

EOWMAN, ALLEN L. $(T-14)$
$52-30$

BOWMAM, J. DAVIZ ( $\because P-4)$ $50-31$
$53-71$

BOMMAS, J. DAVIL $(\mathrm{P}-4)$ COMP. 
BOWMAN, MELVIN G. (CMB-DO)

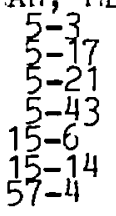

BCYD, $\underset{41-10}{D} P$.

BOYER, K.

BRALBUKY
$2-29$
$2-62$ JAMES N. (MP-3)

BRADLEY $5-46$. C.

BRADLEY
$30-26$
$30-28$ ORVIL D. $(\mathrm{H}-5)$

BRADY, $\underset{18-25}{D}$. N.

BRADY $\frac{\text { JOHN W. }}{50-127}$ JR. (CNC-2)

BRAINERD, WALTER S.

ED.

ERAITHWA ITE, W. J.

$\underset{53-70}{\text { BRAITHWA ITE, WILFRED J. }}$

BRANDENBERGER, JERRY D. (Q-1) 35-6

BRANDOW, BAIRD H. (T-11) $50-66$
$50-125$

BRASIER ${ }_{18-35}$ ROBERT I.

BRAU, CHARLES (AP-2)
$50-30$

BREEDLOVE, JAMES R. (M-8) $41-111$
$41-119$

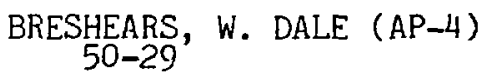 \\ BRITT, ${ }_{53-112}^{\text {HAROLD C. (P-9) }}$ \\ BRITT $_{2-25}$ WILLIAM E. (E-2) \\ BRIXNER, BERLYN (M-5)
$16-22$
$33-6$
$33-7$ \\ BROLLEY, JOHN E., JR. (M-8)
$41-64$
$41-92$
$41-93$
$41-94$
$41-95$
$41-96$
}

$\underset{39-2}{\text { BROMAGE }}$ G. E.

BRONSON, JOHN C. (Q-10)

15-10

$15-11$

$52-10$

$52-18$

$52-23$

BROUSSEA $20-1$, ARMAND T. (CTR-11)

BROWN, J. J ${ }_{3} \mathrm{C}$.

BROWN, MARY C. $(\mathrm{P}-14)$ COMP.

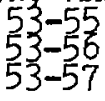

BROWN, RONALD E. (P-7)

$53-6$
$53-113$

BROWN ${ }_{51-32}^{\text {WILBUR K. }}$ (TD-7)

BROWNING, RICHARD V. (WX-3)

$16-27$
$41-41$

BROXTQN 16 DAVID E. (G-5)

$18-16$
$18-47$ 

BRUCKNER, LAWRENCE A. $(Q-12)$
$41-79$
$48-8$
$\underset{48-5}{\text { BRUCKNER, LAWRENCE A. }}$ (S-1)
BRYANT, $18-3 \frac{\text { ERNEST A. }}{4}(\mathrm{CNC}-11)$
BRYSK, HENRY (L-6)
$32-87$
BUCHWALD, MELVIN I. (AP-2)
$32-2$
$32-73$
BUDDING ${ }_{18-33}^{A} \cdot \mathrm{J}$.
BUDEN, DAVID (Q-DO)
$43-1$
$43-2$
BUNKER, MERLE E.
33- $9-12)$
BUNKER, MERLE E. $(P-2)$
BURKE, D. G.
$53-80$
$53-83$
BURKHARDT, LOUIS C. (CTR-2)
$20-23$
BURKHEAD, MARTIN S. (J-15)
$51-36$
BURLESON, G.
$53-70$
BURMAN, ROBERT L. (MP-4)
$53-32$
$53-86$
$53-87$
BURMAN, ROBERT L. (MP-4) COMP.
BUSHNELL, ANDREW $\mathrm{H}$.
$32-2$
$32-73$

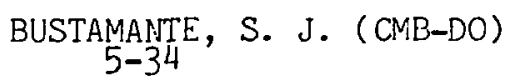
BUSTOS, JOSE M. $(\mathrm{H}-5)$
$30-27$
$\underset{32-17}{\text { BUTCHER }} \underset{\text { ROBERT R. }}{\text { R. AP-2) }}$
BUTLER, MILLARD T. $(\mathrm{H}-10)$
BUTLER, SUSANNAH（L-7)
BUTLER, THOMAS A. $(W X-8)$
BUTTERFIELD, KENNETH! B. (AP-2)
$34-6$
$34-21$
BUZBEE, $\frac{\text { BILLY L. }}{47-78}(\mathrm{C}-3)$
BUZBEE, RILLY L. (C-3) COMP.
BYERS $_{9-2}$ CLEO C. $(Q-14)$
C ? GROUP
CALHOUN $_{18-36}$ T. E.
$\mathrm{CALL}_{39-12} \mathrm{D}^{\mathrm{L}} \cdot(\mathrm{L}-10)$
CAMPBELL CALVIN L. 
CAMPEELL, DAVID K. $(\mathrm{T}-8)$
$53-95$
$54-9$

CAMPBELL, EVAN E. $(\mathrm{H}-5)$
$30-10$
$30-16$

CAMPBELL, KATHERINE $(Q-12)$
$\begin{aligned} & 44-1 \\ & 44-2\end{aligned}$

CAMPBELL, LAURENCE J. (Q-10)
$52-7.6$
$52-66$

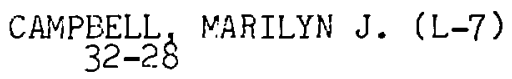

CAMPILLO, ANTHONY J. (AP-4)
$2-60$
$2-61$

CANADA, THOMAS R. (Q-1)

$5-24$
$56-20$

CANA, JOHN $R$.

$2-11$
$2-12$

CANNON, THONAS M. ( $\left.M_{-}-8\right)$

$41-4$
$41-11$
$41-12$
$41-15$
$41-20$
$41-124$

CANTRELL, CYRUS D, , III (L-DOT)
$50-8 O$ $50-80$
$52-16$

CANTRELL, CYRUS D., III $(T-12)$ $34-10$
$50-32$
$50-52$
$50-87$

CANTRELL, EMMETT L. (CTR-3) $20-11$

CARASSO, ALFRED S.

CARLOS, ROBERT C. $(\mathrm{J}-10)$
CARLSON, RANDOLPH L. $(L-1)$ $32-89$

CARLSON,
$52-50$

CARPENTER, JAMES P. $(L-1)$
32-99

CARPENTER, SUSAN G. $(\mathrm{H}-4)$

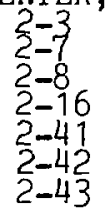

CARSTENS, DEAN H. W. (CMB-3)

$39-6$
$39-25$
$40-1$

CARTER, LELAND L. (TD-6)

CARTER, WILLIAM $\mathrm{J},(\mathrm{G}-7)$

46-11

48-10

$48-11$
$48-12$

CARTER, WILLIAM J. (G-7) COMP.

46-4

$46-5$

$46-6$

$46-7$

$46-9$

48-2

CAFTER, WILLIAM J. (M-6)

CARTWRIGHT, DAVID C. (T-12) $50-398$ $51-55$

CARWILE, CLIFTON

CASHWELL, EDMOND D. (TD-6)

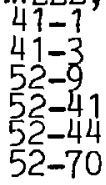




$$
\begin{aligned}
& \text { CASPERSON, D. E. }(L-1) \\
& 32-89 \\
& 32-90
\end{aligned}
$$

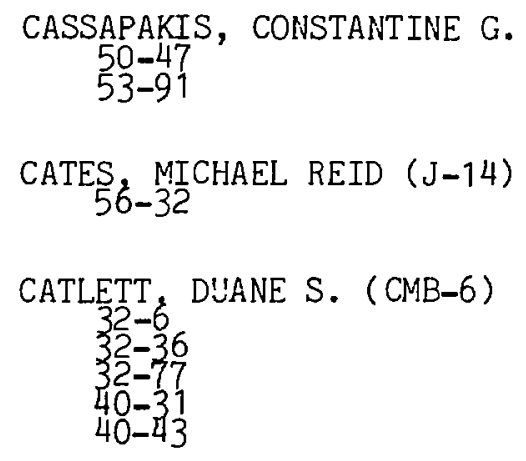

CASSAPAKIS, CONSTANTINE G. $50-47$
$53-91$

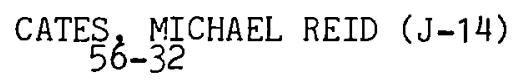

CATLETT, DUANE S. (CMB-6)

$$
\begin{aligned}
& 32-6 \\
& 32-36 \\
& 32-77 \\
& 40-31 \\
& 40-43
\end{aligned}
$$

CAYTON, THOMAS (CTR-6)
T8-4

CHANDLER, GEORGE I. (CTR-8)

$\underset{18}{\text { CHANEY, DAN }}$ S. $(G-5)$

CHAPIN, CHARLES E.

CHA IRMAN

CHARLES 34 ROBERT $W .(\mathrm{CNC}-11)$ $24-2$

29-1

CHAVEZ, J.

CHEADLE $\underset{41-34}{\text { JESSE M. }}$ (C-5)

CHEADLE, JESSE M. $(\mathrm{Q}-12)$

CHENG ${ }_{53-74}^{T}$

CHORN, GRANVILLE E. (C-4)

CHOWDHURI, P. (Q-10) COMP.

$\underset{11-5}{\text { CHOWDHURT, P. (Q-26) }}$

CHRISTMAN, RONALD D. (C-4)

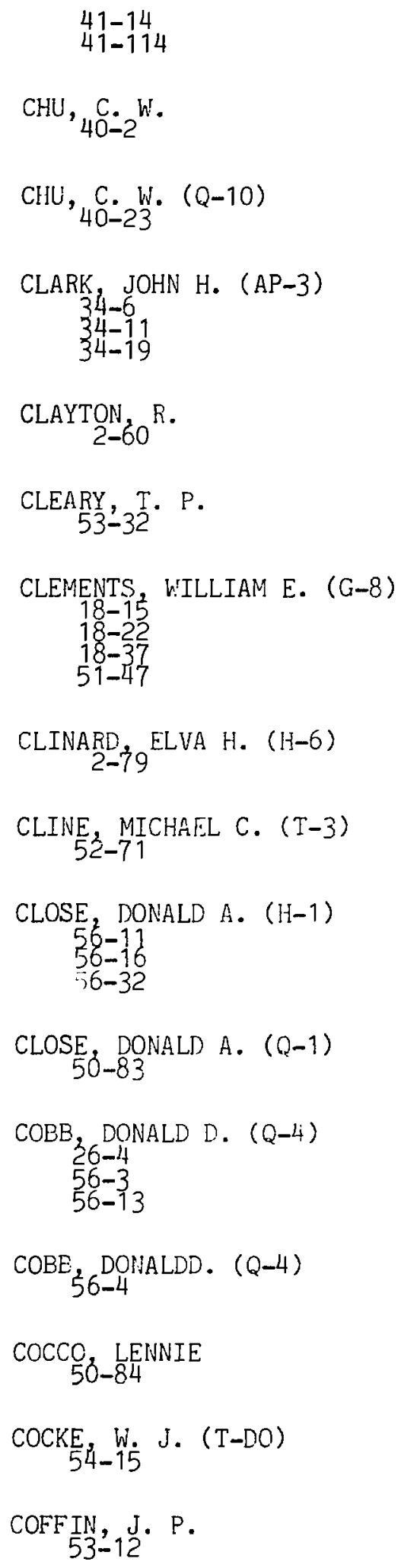


COHEN, JAMES S. (T-12)

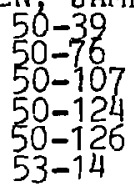

COKER, WILLIAM $R$.

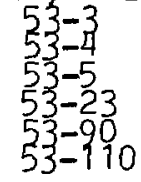

COLE
$\begin{gathered}30=15 \\ 20-28\end{gathered}$

COLGATE, STIRLING A. (T-DOT) $16-37$
$51-48$

COLLIER $\frac{1}{31-15}$ ROBERT K. (Q-11) $52-12$ $57-5$

COLLINS

COLLINS, LEE A. (T-4) $50-44$
$50-69$
$50-70$
$50-124$ COLLINS MAURICE W. (C-4)
$\begin{aligned} & 41-14 \\ & 47-63\end{aligned}$

COLYER, DUARD B. $(Q-10)$

COMLY, JACK C. $(T-6)$

COMMISSO, ROBERT J. (CTR-3)
$19-11$
$20-15$

CONNER, JERRY P. $(P-4)$

CONRAD, ROSEMARY (CTR-8)

$19=4$
$\mathrm{COOK}_{52-47}$ THOMAS L. $(\mathrm{J}-15)$

COOK $_{51-51}$ THOMAS L. $(J-9)$

COOK $\underset{41-55}{\text { WILLIAM A. }}(Q-13)$

COOK WiLLIAM A.
$\substack{41-44 \\ 59-2}$

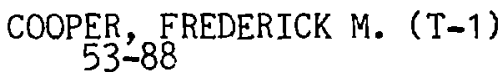

COOPER, FREDERICK M. (T-8) 54-13

COOPER, MARTIN D. $(M P-3)$

CORCORAN, WILLIAM F. (WX-8)

CORRELL, FRANCIS D. (P-7) $53-6$
$53-113$

CORT, GEORGE E. (WX-4)

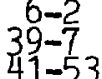

CORTEZ, JOSEPH R. (H-1) $30-6$

$30-20$
$30-22$

COTTINGAME, $W$. 53-70

COULTER $_{40-9}$ C. ALTON (MP-DO)

COUTURES, JEAN-PIERRE
$57-7$

COWAN, GEORGE A. (CNC-DO)

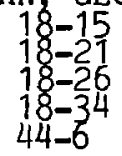

COWAN, GEORGE A. (CNC-DO) ED. 
COWAN, ROBERT D.

COWAN, ROBERT D. $(T-4)$

COWAN $_{5-34}$ ROBERT E. (E-2)

COX, ARTHUR N. (T-DOT)

$51-2$

$51-3$

$51-34$

$51-40$

COX, KENNETH E. (CMB-3)

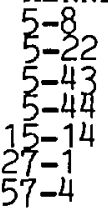

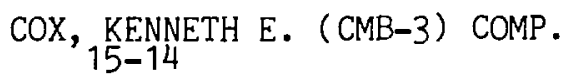

COX, ROBERT A. (H-10)

$2-41$
$2-42$

COY, $\underset{30-17}{\text { DALE E. (E-5) }}$

$\mathrm{CRAIG}_{3-2}$ BOBBY GLENN (E-5)

CRAIG $_{3-7}$ BOBBY GLENN (M-DO)

CRAM, L. SCOTT $(\mathrm{H}-10)$

$2-75$
$50-116$

CRANE, T. W. (Q-1)

$56-2$
$56-18$

CRANFILL,
$32-40$
$32-78$ BETTY S. (L-7)

CRANF ILL, CHARLES $W .(L-6)$
CRISCUOLO, ALPHONSE L. (E-5)
41-27

CRISSMAN, HARKY A. $(\mathrm{H}-10)$

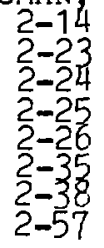

CROMER, DON T. (CME-5)

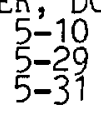

CROWE, BRUCE M. (G-6)

$18-42$
$45-12$

CROWE ${ }_{18-2}$ BRUCE M. (Q-21)

CROWELLL, JOHN M. $(H-10)$

$\underset{32-60}{\text { CUMNIINGS CHARLES E. }(W X-4)}$

CURTIS, DAVID B. $(\mathrm{H}-8)$

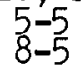

CZUCHLEWSKI, STEPHEN J. (L-9)

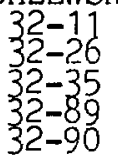

D'ANNA, JOSEPHI A. (H-9)

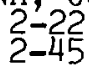

$\underset{52-62}{\operatorname{D} A R R U D A}$ JOSE

D'LPPOLITO, D. A.
$39-19$

D 'ANINA, JOSEPH A. (H-9)

DA PAIXAO, FERNANDO J. 


$$
\begin{aligned}
& \text { DABBS, J. W. T. } \\
& 53-100 \\
& \text { DAHL } \begin{array}{c}
\text { DAVID A. }(H-8) \\
41-62 \\
56-26
\end{array}
\end{aligned}
$$

DALTON, CHARLIE G. (MP-4) $41-8$
$53-41$

DALY $_{35-5}$ BART J. $(T-3)$

DANEN, WAYNE C. (AP-3)

DANIELS, REBECCA J. (CNC-11)
$2-72$
$53-48$

DANIELS, WILLIAM R. (CNC-11)

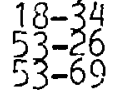

DARDEN, C. W. $53-32$
$53-87$

DASIIEN,

DATLA, ${ }_{19-17}^{R .} U$.

DALE, $\underset{5-41}{\text { GUIDO H. }}$

DAVID $_{5-17}$ MARY ANN (CMP-3)

DAVIDS, CARY $N$

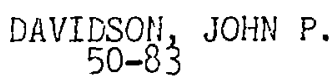

DAVIDSOH, KEITH V. (CMB-6)
$25-4$

DAVIE.S, T. J .

$$
\begin{aligned}
& 16-8 \\
& 16-9 \\
& 16-10
\end{aligned}
$$

DAVIS, CECIL G. (J-15)
$51-49$
$51-50$

DAVIS, Ti-5j NEIL

DAVIS, THOMAS O., JR. $(\mathrm{H}-5)$

DAVISON, DAVID $K$

DAY, $\underset{32-60}{\text { ROBERT D. }}(\mathrm{E}-4)$

DAY, $\underset{32-57}{\operatorname{ROBERT} D .(L-1)}$

DAY, ROBERT H. $(J-14)$

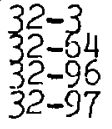

DAYEM, HASSAN ALY (Q-4)

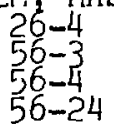

DE FIELD, JAMES D. $(\mathrm{H}-5)$

DE LISI 20 CHARLES P. $(T-10)$

DE POORTER, GERALD L. (AP-3) $4-1$
$50-89$

DE POORTER, GERALD L. (AP-4)

DE SILVA, ALAN! W.

DE VAULT, GUILLAUME P. (Q-7)

DEAN $_{30-21}$ BRENDA J. $(\mathrm{H}-5)$

DEAVEN, IARRY L. (H-9)

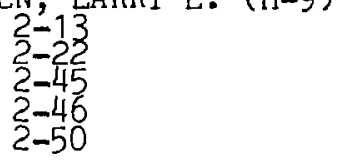


DEBUSK, THOMAS P. (CNC-11) $53-44$

$\mathrm{DEMBO}_{2-4 \mathrm{MICAH}}(\mathrm{T}-10)$ $2-44$

$2-83$

DEMPSEY $18-12$ THOMAS

DEMUTH, ANNE (AT-3)

$41-33$

DEMUTH, RUTH B. (T-3)

DENNIS, BERT R. (G-4)

$$
\begin{aligned}
& 21-3 \\
& 24-6
\end{aligned}
$$

DENNIS, BERT R. (Q-22)

DENTON, WILLIAM H. $(Q-10)$
$39-8$

DEPP $\underset{16-13}{\text { STEVEN W. }}$ (E-DO)

${ }_{27-16}^{\text {DEROUIN }}$ CHARLES (E-DO)

DEUPREE

DEUPREE $\underset{51-19}{\text { ROBERT G. (T-DOT) }}$

DEVANEY, JOSEPH J. (L-5)

$$
\begin{aligned}
32-39 \\
32-49
\end{aligned}
$$

DEVERALL, JOHN E. (Q-13)

DEVEREUX
$53-33$
$53-70$$\quad$.

DIAMOND, STEPHEN C. $(W X-4)$ $16-4$
$31-2$
$31-18$
DICELLO JOHN F., JR. (MP-3)
$2-8$ -
$2-29$
$2-40$
$2-49$
$2-74$
$52-58$

DICK, JERRY JOEL (M-3)

$52-74$

DICKINSON, JAMES M. (CME-6)
$20-7$
$40-13$

DICKMAN, DONALD O. (L-5)

DIENES, JOHN K. (T-3)

$18-39$
$50-75$

DIERCKX, R.

$17-2$
$53-73$

DIETZ, RUDOLPH J., JR. (Q-4)

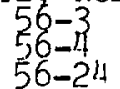

DIKE, ROBERT S. (CTR-4) $20-9$ 20-23

DINEGAR, ROBERT H. (WX-7)<smiles>[As]1[As][As]1</smiles>

$\underset{52-66}{\text { DISATNIK }}$ Y. (Q-10)

DIXON, THOMAS L. (WX-3)

DOLL, JIMMIE D. (CNC-2) $50-50$
$50-127$

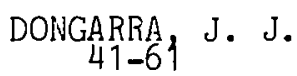

$\underset{41-110}{\text { DONGARRA }}$ JACK

DOOLEN, GARY D. (TD-2) 
$50-43$
$53-78$

DORNING 38 J. J.

DORR, $\underset{41-23}{\text { FRED W. }}$ (C-DO)

DOSS, JAMES D. (MP-3)

$2-32$
$2-85$
$2-87$

$\underset{56-29}{\text { DOUGHERTY, JOHN E. (TD-7) }}$

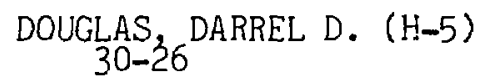

$\underset{30-27}{\text { DOUGLAS, DAREL D. }(H-5) \text { PROJECT MANAGER }}$

DCVE $_{\{6-2}$ RICHARD C. $(Q-13)$

DOWDY EDWARD J. (Q-2)

DOYLE, B. 32.

DRAKE, DARRELL M. (P-3) $53-45$

DRAKE 3 GLESSIE A. (H-4)

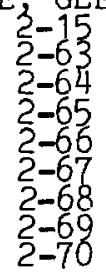

$\underset{18-14}{\operatorname{DREESEN}} \underset{14}{\text { DAVID R. }}(\mathrm{H}-12)$

DREESEN
$18-64$

$\underset{58-7}{\text { DREICER, HARRY (CTR-DO) }}$

$\underset{50-91}{\text { DROPESKY }}$ BRUCE J. (CNC-11)
$53-34$

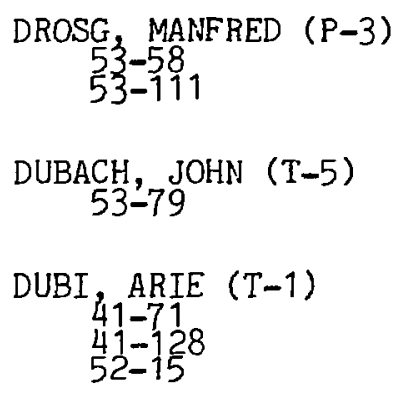

$\underset{26-23}{\text { DUDZIAK }}$ DONALD J. $(T-1)$ COMP. DUERRE, KENNETH H. (WX-8) $16-2$
$16-25$
$41-24$

DUFFY $\underset{18-65}{\text { CLARENCE J. (CNC-11) }}$

$\underset{32-70}{\text { DUNLAVY DONALD J. }}(E-10)$

DUNN , ELEANOR D. (MP-DO) COMP. $47-17$
$47-18$
$47-19$

DUNNING ${ }_{50-22}$ THOMAS H., JR.

DUNNING, THOMAS H., JR. (CNC-2) $50-21$
$50-38$
$50-123$

DUNWOODY WADE E. (Q-13) 


$$
\begin{aligned}
& \text { DURAND, LOYAL (T-DO) } \\
& \text { 41-98 } \\
& \text { DURHAM, FRANKLIN P. (L-DO) } \\
& \text { 32-61 } \\
& \text { EASTMAN, TIMOTHY E. } \\
& 51-6 \\
& \text { ECCLESTON, GEORGE W. (Q-1) } \\
& 56-16 \\
& \text { ECKHARDT, ROGER C. (AP-2) } \\
& 34-5 \\
& \text { EDESKUTY, FREDERICK J. (Q-10) } \\
& 11-1,15-4
\end{aligned}
$$

EDESKUTY, FREDERICK J. (Q-10) COMP. $11-2$
$14-1$

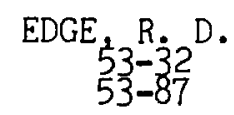

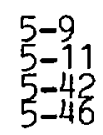

$\underset{32-8 .}{\text { ELLIOTT }}$ C. JAMES ( $\mathrm{L}-6)$

$\underset{15-1}{\text { ELLIOTT }}$ GUY R. B. (CNC-2)

ELLIOTT, GUY R. R. $(G-7)$

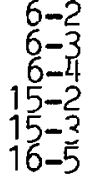

ELLIOTT ${ }_{41-16}^{\text {RAYMOND L. (C-6) }}$

$\underset{53-109}{\text { ELLIS, ALBERT N. }}(\mathrm{P}-3)$

ELLIS, WALTON P. (CMB-8) $40-3$
$40-39$
$40-21$
$40-34$

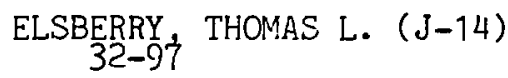

ELSNER, DORIS B. (G-3) COMP.

EMANUEL, GEORGE
$54-3$

EMANUEL, GEORGE (AP-DOT)

EMIGH $_{53-7} \underset{3}{C}$ ROBERT $(P-14)$

ENDEBROCK, ELTON G. ( $\mathrm{WX}-8)$ $18-35$
$18-40$
$41-28$ $\underset{33-4}{\text { ENDEBROCK, MARJORIE }}(E-10)$ COMP.

$\underset{50-24}{\text { ENGELKE }}$ RAY (M-3)

ENGLAND, TALMADGE R. (T-2) 


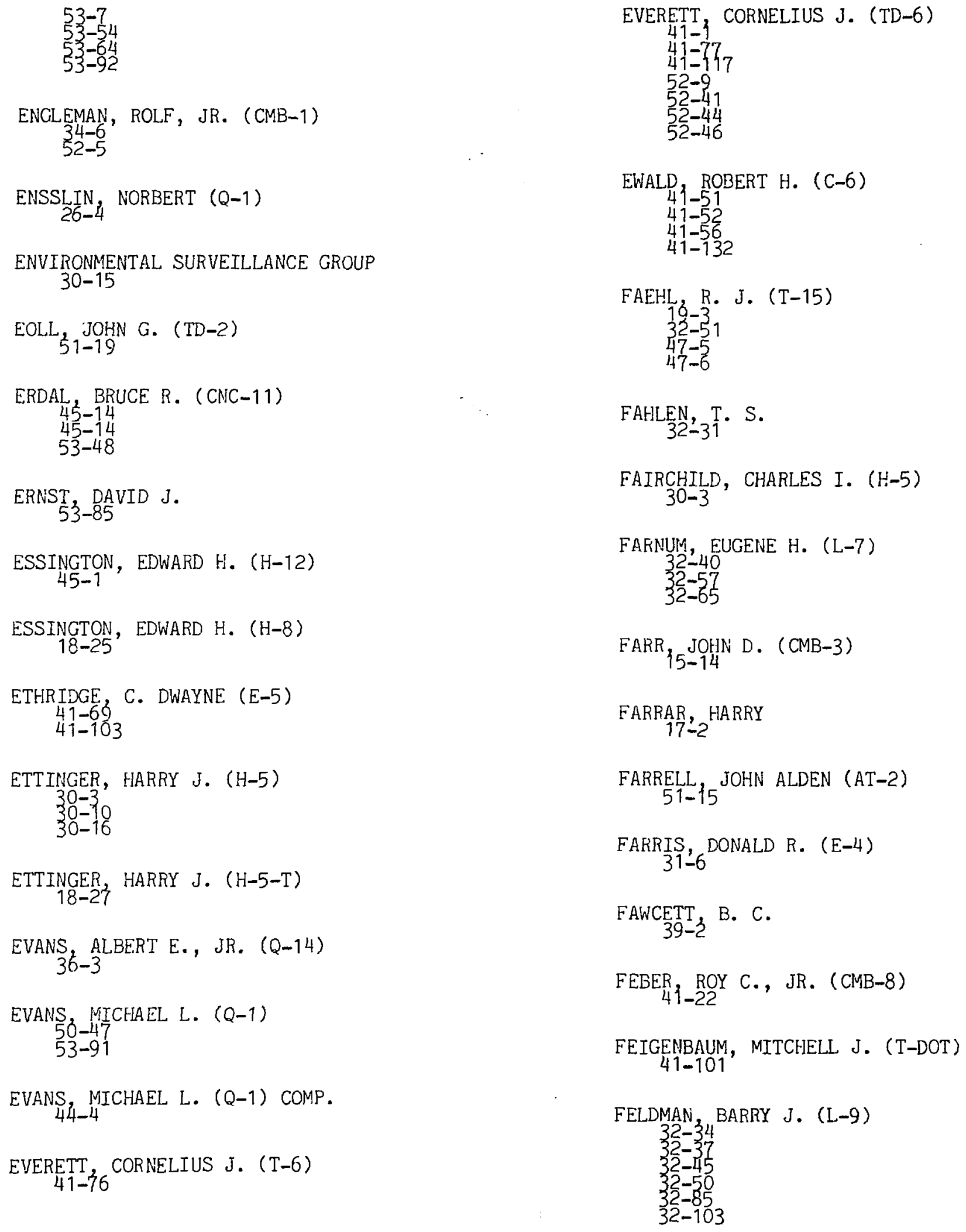


FELDMAN, WILLIAM C. $(P-4)$

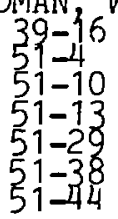

$50-98$

FLORIN, ALAN E.

FLYNN, EDWARD R. (P-7) $50-118$

FELTHAUSER, HARRY E. (P-4) $51-25$
$51-26$

FENIMORE, EDWARD (P-4)

$$
\begin{aligned}
& 33-3 \\
& 41-11 \\
& 41=12 \\
& 41-124 \\
& 51-38
\end{aligned}
$$

FLYNN, EDWAFD R. (P-9)

$$
\begin{aligned}
& 53-12 \\
& 53-83 \\
& 53-98
\end{aligned}
$$

FOLEY, EDW'AFD (L-9)

$$
\begin{aligned}
& 32-11 \\
& 32-26 \\
& 32-35 \\
& 32-93
\end{aligned}
$$

FICUEIRA, JOSEPH F. (L-9)

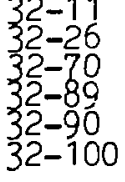

FILIP, HENRY (AP-3)

FILIP, HENRY (AP-4)
$50-55$
$50-56$

FINCH, FRANCIS T. (AP-DOT) COMP. $32-95$

FORD, AIILREN (S-2) $37-18$
$41-108$

FORD , GEORCE P. ( $\mathrm{CNC}-11)$ $53-13$

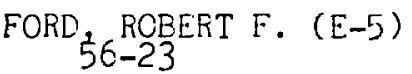

FOREST, ${ }_{3-1}$ CHARLES A. $(T-14)$

FINK, CONRAD F. (G-4)

$33-1$
$23-2$

FORSLUA:D, DAVIE $h \cdot(L-6)$

FISHER, ROBERT A. (L-9)

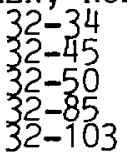

$\underset{2-75}{\text { FCRSLUND, }}$ CEAFi C. $(\mathrm{H}-10)$

FOWLER, ERIC [s. $(\mathrm{H}-12)$

FCWLER, ERIC B. $(H-8)$

FOWLER, ${ }_{54}$ G. N.

FOWLER, MALCOLM M. $\quad(\mathrm{CNC}-11)$

FOWLER, MALCOLW $\mathrm{M} .(\mathrm{CNC}-11)$ COMP. 
FOX, KENNETH

FOX, $\underset{50-32}{\text { KENNETH }}(\mathrm{AP}-4)$

FRALEY, GARY S. $(L-6)$

FRANCE, STEPHEN W. (Q-2)

FRANK $3-8.8 \cdot(H-10)$

FRALK, THURMAN G. $(L-5)$ $20-14$
$32-18$
$32-39$
$32-44$

FRANKS,

FRAYKS, L. A.
$56-32$

FRASER, JOHN S.

FREESE, KENNETH E. (CTR-8)

FREESE, KENNETH B. (CTR-8) COMP.

FREIDBERG, JEFFREY P. (CTR-5) $39-19$
$58-5$
$58-11$

$\underset{27-13}{\text { FREIWALE, DAVID A. (DIR OFF) }}$

FREIWALD, DAVID A. (DIR-O)

$$
\begin{aligned}
& 27-14 \\
& 28-5 \\
& 32-9
\end{aligned}
$$

$\underset{27-2\}}{\text { FREIWALD, DAVID A. (DIR-O) COMP. }}$

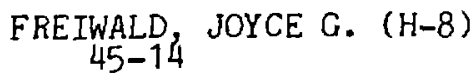

FREUND, SAMUEL M. (AP-3)
$34-17$

FREUND, SAMUEL M. (AP-4) $34-1$
$34-16$
$34-18$

FREYMAN $16-13$ ROBERT '. (TD-7)

FRIAR, JAMES 1. (T-5) $52-45$

$53-76$

FRICKE, GERHARD

FRIES, RALPH JAY (L-7) $32-75$
$32-76$

FRITZ, GEORGIA T. $(W X-2)$

FU, JERRY $\mathrm{Ji}-53(\mathrm{WX}-4)$

FUKA $\underset{15-10}{\text { LOUIS R. (Q-26) }}$

FUKUSHIMA, EIICHI (CNC-4)
$50-42$

FULLBRIGHT, HENRY J., III $(M-1)$

FULLERTON, L. WAYNE $(\mathrm{C}-3)$

$\underset{53-87}{\text { GABATHUL,ER, } K .}$

GAC, FRANK D. (CMB-6) $40-17$
$40-22$

GALBRAITH, HAROLD W. (T-12) 50-10 $50-13$ $50-18$ $50-52$
$50-87$ $50-115$

50-128 
GALLECOS, ANTHONY $\mathrm{F} .(\mathrm{H}-12)$

GANLEY, JAMES T. $(L-10)$

$\begin{array}{ll}3 & -5 \\ 32 & -54 \\ 32 & -81\end{array}$

GARCILAZO, HUMBERTO (T-5)

GARDE, RAYMOND $(\mathrm{H}-1)$

GARY S. $_{514}$ PETER (CTR-6)
$58-1$
$58-2$

GARY $_{39-16} S_{i 6}$ PETER $(P-4)$

GAVRON, AVIGDOR I. (P-9) 53-112

GEACINTOV, N. E.
$2-61$

GEDAYLOO, TEYMOOR

GEESAMAN, D. F. 53-33

GENTER, $F . C$. $2-5$
$48-5$

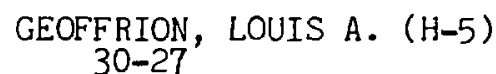

GEORGE, DENISE C. (T-2) 53-7

GERMAIN, LAWRENCE S. (J-DOT)

18-33- 43

GERSTL, S. A. W. (T-1)

$41-71$
$41-128$

GETTEMY DONALD J. $(\mathrm{H}-5)$

$$
2-39
$$

GIBBS, WILLIAM R. (T-5)

GIBSON, A. A. V.
$50-42$

GIBSON, BENJAMIN F. (T-5)

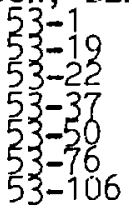

GIDO, RICHARE G. (Q-6)

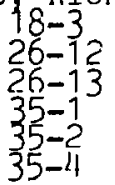

GIESLER,
$\begin{gathered}41-84 \\ 50-91\end{gathered}$

$\underset{51-31}{\text { GIGUERE }}$ PAUL T. $(T-4)$

GILBERT, JOEL S. (Q-6) $18-3$
$35-2$

$\underset{18-25}{\text { GILBERT }}$

GILL $\begin{aligned} & 32-20 N I S ~ H . \\ & 42-(A P-2) \\ & 41-126\end{aligned}$

${ }^{\text {GILL }} 16-i 4$

GTNOCCHIO, JOSEPH N. $(T-5)$ $53-25$
$53-53$

GIOPGI, ANGELO L. (CME-3)

40-10

40-20

$40-38$

$40-46$

$\underset{32-86}{\text { GIOVANIELLI, DAMON }}$ V. $(L-4)$ 
GITGMER, STEVEN J. (L-6) $32-87$
$32-8$

$\underset{32-5 \varepsilon}{\text { GITONER }}$ STEVEN J. $^{(T-6)}$

GLADNEY, ERNEST S. $(\mathrm{H}-8)$

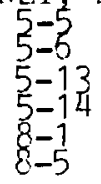

GLASHAUSSEF, $C$. $53-3$
$53-5$

GLASS, $\mathrm{G}$.

$53-60$
$53-91$
$53-104$

GLASS, ${ }_{50-47} \cdot(P-D O R)$

CLORE, JAMES P. (P-4) $51-25$
$51-26$

GLUCESTERN, ROBERT L. (MP-8)

GOAD, WALTER B., JR. $(T-10)$

GODFREY, ERENDAN B. $(\mathrm{T}-15)$ $19-3$
$47-5$
$47-6$

GODNIIN, ROBERT P. (L-4) $32-38$

$\underset{39-19}{\text { COEDBLOED, J. P. }}$

$\underset{18-29}{\text { GOFASFR F. }}(G-6)$

GOFORTII, JAMES $\mathrm{H} .(w \mathrm{~T}-7)$

GOLD, R.

GOLDHABER, ALFRED S. (T-5)
$53-43$

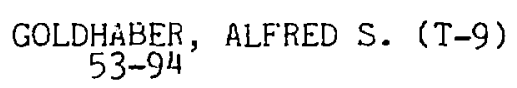

GOLDMAN WARTIN VAN
$50-92$

GOLDMAN, TERRENCE J. (T-8)

$50-90$
$54-17$

GOLDSTEIN, BYRON $(\mathrm{T}-10)$

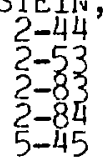

GOLCSTEIN, JOHN C. (L-6)

GOLDSTONE, JOYCE A. (CME-8)
$40-8$
$53-20$

GOLDSTOHE, PHILIP D. $(L-1)$
$50-61$

$\underset{53-112}{\text { GOLDSTONE, PHILIP D. }}$ (P-9)

$\underset{16-9}{\text { GOLOB }}$ JOHN E.

GOLOB $_{10-10}$ JOHN E. (E-DOR)

GOLOB, JOHN E. $(E-3)$
$16-8$
$52-24$

GOMEZ, MARY FRANCES (T 1$)$ $26-22$
$26-24$
$26-25$

GOMEZ, MARY FRANCES $(T-1)$ COMP.

GOOLEY, RONALD C. $(G-6)$

GORDON, GLEN E.

GORE, RAYMOND A. (E-DO) 


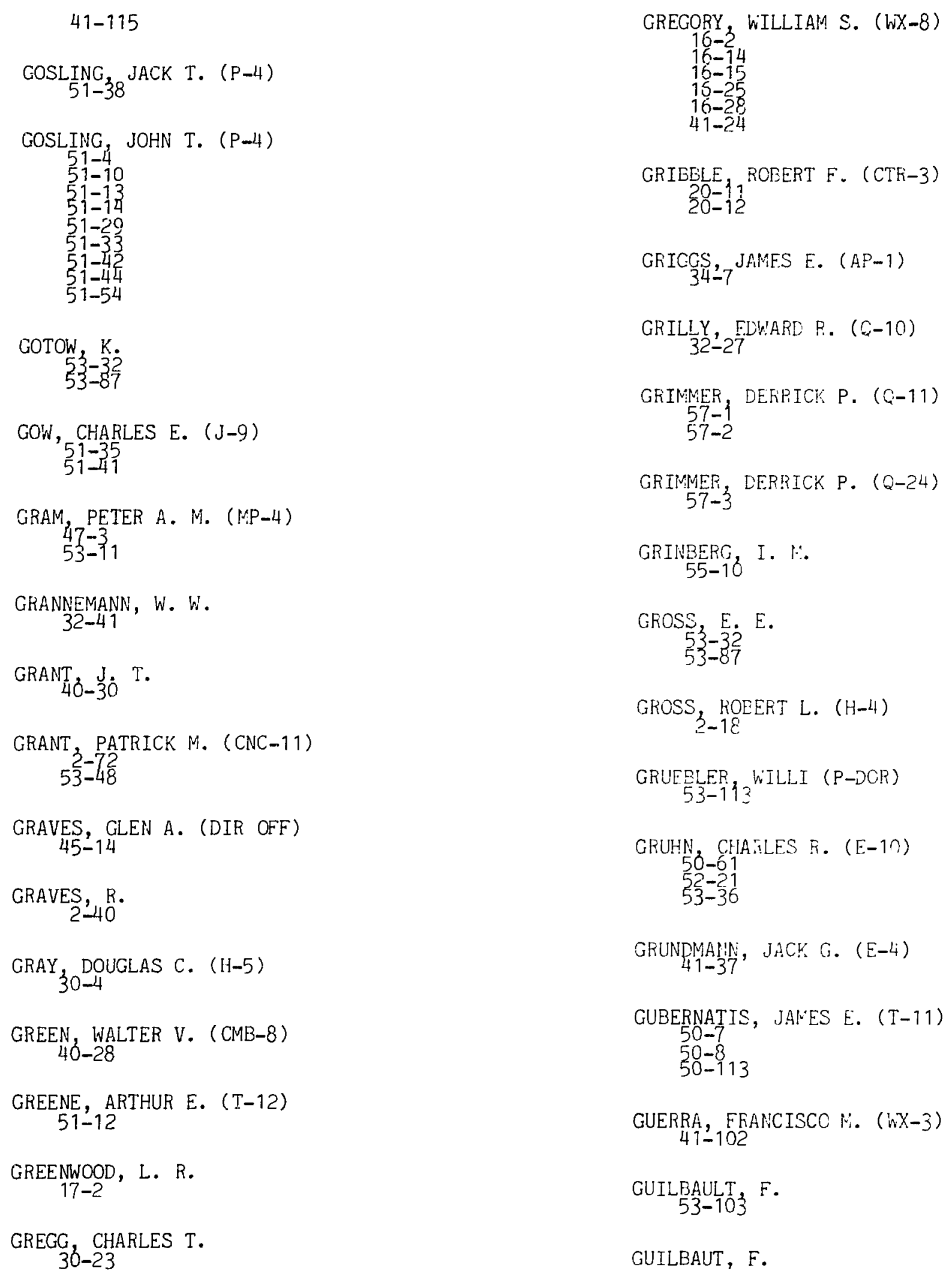




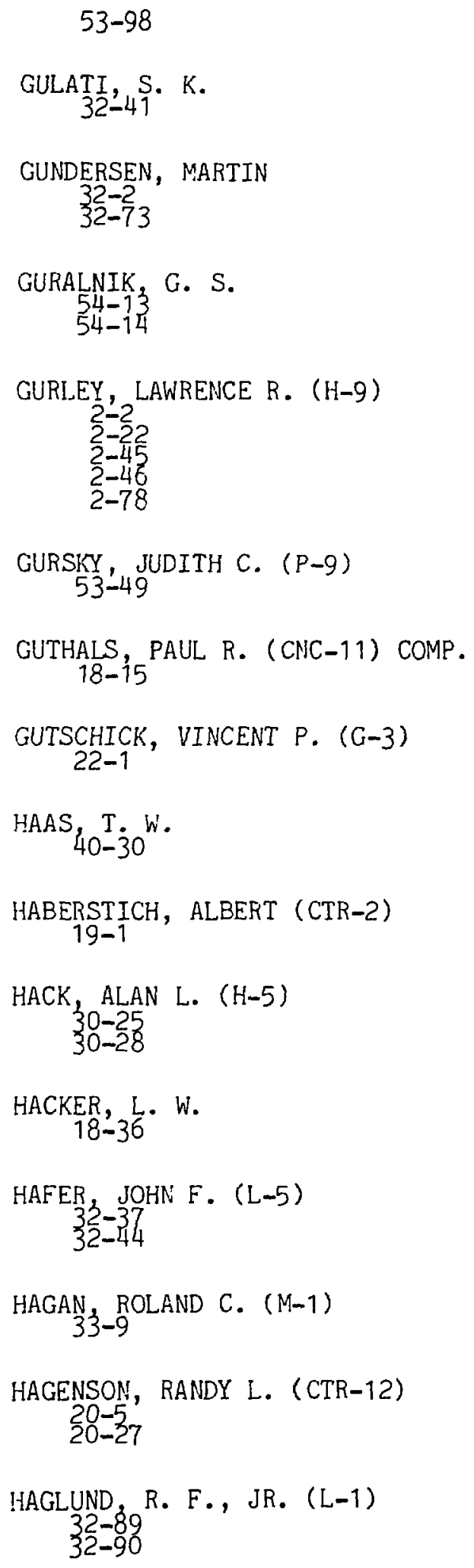

HAGLUND, R. F., JR. (P-9)
$53-6$
$53-96$

HAIRE, RICHARD G.

HAKKILA
$56-16$
$56-24$ . ARNOLD (CMB-1)

HAKKILA, E. ARNOLD (Q-4)

$56-3$
$56-4$
$56-5$

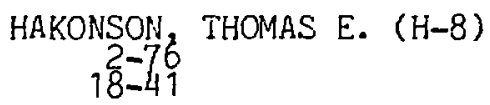

HALBIG, JAMES K. (MP-9)

HALL $_{27-11}$ JAMES W. (AADP-DO)

HALSTEAD, CORDON W. (CNC-4)

HAMM $\underset{47-7}{\text { ROBERT W. }}$ (MP-12)

HAMMEL, EDWARD F., JR. (DIR OFF)

HAMMER, CHARLES F. (CTR-4)

HAMMOND $\frac{5-1}{5-4}$ C. F. (CMB-1)

$$
\begin{aligned}
& 5-3 \\
& 5-4
\end{aligned}
$$

HAN, KI S. (J-12)

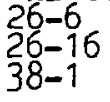

HANKS, KENNETH W. (CTR-4)

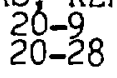

HANSBOROUGH, LASH D. (AT-1)

HANSBOROUGH, LASH D. (MP-14) $20-7$
$20-15$ 
HANSEL, JAMES M. ( CME-1) $5-15$
$5-16$

HANSEN, GORDON E. (Q-14) $9-2$
$26-17$

HANSEN, KAREN M. (H-10)

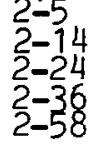

HANSEN, OLE

HANSEN, WILFRED G. (L-1) 32-57

HANSON, DAVID L. (P-7) $50-118$

$53-81$
$53-103$

HANSON, DAVID L. $(P-9)$

HANSON, KENNETH M. (MP-3)

$$
\begin{array}{r}
2-27 \\
2-73 \\
41-10
\end{array}
$$

HANSON, WAYNE C. $(\mathrm{H}-12)$ 18-11

HARDEKOPF, ROBERT A. (P-7) 53-6

HARDEKOPF, ROBERT A. (P-9) 53-96

HARLOW, FRANCIS H. (T-3)

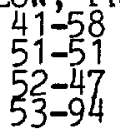

HARLOW, FRANCIS H., JR. (T-3)

HARMON, ANTHONY W. (CMB-1) $8-8$

HART, VALGENE E. (MP-8) 47-16
HARTER, WILLIAM G.
$50^{2}-48$

HARVEY, ALEXANDER (MP-13)

HASSENZAHL, WILLIAM V. (Q-10)

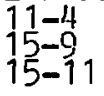

HASSENZAHL, WILLIAM V. (Q-10) COMP. $15-10$
$15-11$

HASSENZAHL, WILLIAM V. (Q-10) ED. $47-30$

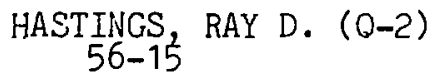

HATCHER $_{56-19}$ CHARLES R. $(Q-1)$

HAUTH, WILLARD E. (CMB-6) $40-13$
$40-22$

$40-41$

$40-42$

$\mathrm{HAWK}_{51-25} \stackrel{\mathrm{H} .}{\mathrm{L}}$.

HAXTON, LAURA K. (H-DO)
$30-30$

HAXTON, WICK C. (T-5)

$53-79$
$53-105$
$53-107$

HAY, P. JEFFREY (T-12)

$50-3$

$50-2$

$50-22$

$50-37$

$50-38$

$50=110$

HAYES, $F_{2-20}$ NEWTON (H-9)

HDR $\underset{24-5}{\text { PROJECT STAFF }}$ 
HEALY $\frac{30-24}{\text { JOHN W. }}$. (H-DO)

$\mathrm{HECHT}_{50-12} \underset{\mathrm{H}}{\mathrm{H} R R Y} \mathrm{G} \cdot(\mathrm{CNC}-2)$

HECKER, SIEGFRIED S. (CMB-5)

HEDSTROM, JAMES C. (Q-11)

$$
\begin{aligned}
& 31-4 \\
& 31-10 \\
& 31-11
\end{aligned}
$$

HEFFNER $\underset{50-\delta 1}{50 B E R T ~ H . ~(M P-3) ~}$

$$
\begin{array}{r}
50-81 \\
53-86
\end{array}
$$

HEIKEN, GRANT (G-6)

$$
\begin{aligned}
& 18-42 \\
& 45-12
\end{aligned}
$$

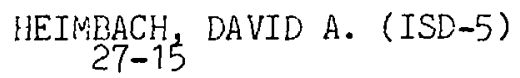

HEINRICH, R. R.

HELLAND $2-29$ JERONE A. (MP-3)

HELMICK 3 HERBERT H. (Q-3)

$$
\begin{aligned}
& 25-3 \\
& 26-9
\end{aligned}
$$

HEMMERIDINGER, AFTHUR (P-3)

$$
\begin{aligned}
& 53-15 \\
& 53-109
\end{aligned}
$$

HEIMERICH,
$51-26$.

HEMPELMANN, LOUIS H. (H-DO)
$30-30$

$\underset{32-71}{\text { HEIDERSON, DALE B. }(L-6)}$

HENDERSON, RICHARD W. $(H-1)$
$30-17$

HENDRICKS, JOHN S. (TD-6)
$56-11$

HENDRON, ROBERT H. (G-4)
HENNINGER, RUDOLPH J. (T-1)

$$
\begin{aligned}
& 36-1 \\
& 39-17
\end{aligned}
$$

HENRY $\underset{56-15}{\text { CARL N. }}$ (Q-2)

HENSLEY, WALTER $K \cdot(P-2)$
$5-11$
$8-11$

HERMES, ROBERT E. $(\mathrm{H}-5)$

HERCLD, R.

HERR, KENNETH C.

$$
\begin{aligned}
& 57-1 \\
& 57-3
\end{aligned}
$$

HERRICK $_{5-34}$ CLAUDE C. ( CMB-8)

HERRICK ${ }_{5-34}$ G. C.

HEWETT, DENNIS W. (CTR-6)

HIEBERT, J. C.
$50-47$
$53-97$
$53-104$

HIEBERT
$2-19$
$2-74$

$\underset{2-5}{\mathrm{HIEBERT}} \underset{2}{ }$ RICHARD D. (E-5)

HIGBIE, PAUL R. (P-4)

$$
\begin{aligned}
& 51-20 \\
& 51-21 \\
& 51-22 \\
& 51-30
\end{aligned}
$$

HILDEBRAND, CARL E. (H-9)

$$
\begin{aligned}
& 2-2 \\
& 2-45 \\
& 2-78
\end{aligned}
$$

HILL, DWIGHT E. (G-5)

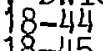


$\mathrm{HILL}_{24-8}$ JAMES H. (G-4)

$\mathrm{HILL}_{38-4}$ JAMES H. $(Q-22)$

HILL $_{53-100} \mathrm{~N}$

HINKLEY, E. DAVID
$50-52$

HINMAN, GEORGE W.
$18-28$

HINSLEY, RONALD (AP-2)

HINTZ, N. M.

$53-3$
$53-5$
$53-90$

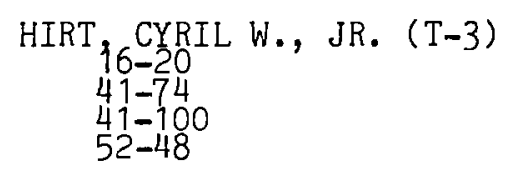

HOARD 3 DONALD E. (H-9)

HODSON, STEPHEN W. (T-DOT)

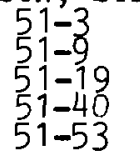

HOEHN, MARTHA V. (P-2)

$50-71$
$53-68$

HOFFMAN, CARROLL G. $(\mathrm{L}-7)$

HOFFMAN, CYRUS M. (MP-4)
$54-17$

HOFFMAN, DARLEANE C. (CNC-11)

$45-14$
$53-26$

$53-26$
$53-72$

$\underset{52-35}{\text { HOFFMAN }}$ NELSON M. $(\mathrm{J}-15)$ $\underset{2-25}{\text { HOFFMAN }_{3} \text { ROBERT A. }}(\mathrm{H}-10)$

HOFEMANN, G. W. $(M P-10)$

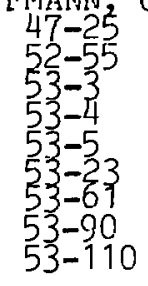

HOHMANN, PHILIP G. $(\mathrm{H}-9)$
$2-45$
$2-78$

HOLIAN, BRAD LEE $(T-11)$
50-78

$\underset{25-2}{\text { HOLLABAUGH, CHARLES M. (CMB-3) }}$

HOLLAND, LAURENCE M. (H-4)

$$
\begin{aligned}
& 2-63 \\
& 2-64 \\
& 2-67 \\
& 2-77
\end{aligned}
$$

HOLLAND REDUS F. (AP-4)
$34-16$
$34-18$
$50-55$
$50-72$
$50-97$

HOLLAS, C. L.

HOLLEN, $\underset{5-20}{\text { ROBERT M. }}$ (CMB-1)

HOLLEY, CHARLES E., JR. (CNC-2)

$$
\begin{gathered}
5-2 \\
5-18 \\
16-5
\end{gathered}
$$

HOLLSTEIN, ULRICH

HOLM $_{33-8}$ DALE. M. $(H-6)$

HOLMES, VANNER H., JR. $(J-12)$

HOLT $_{53-86} \mathrm{ROY} \mathrm{J}$ 
HOMUTH, EMIL F. (G-2)

HONES, EDWARD W., JR. (P-4)

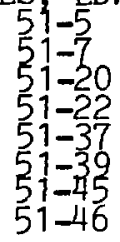
HONEY $5 \delta_{-32}$ FRANCIS $\mathrm{J} \cdot(\mathrm{J}-8)$

HONEYCUTT, R. KENT $51-35$
$51-41$

$\mathrm{HONG}_{32-32}$ JUNG PYO (L-10)

$\underset{7-4}{H O P K I N S}$ D. N. (G-7)

HORAK, HENRY G. $(J-10)$ $33-1$
$51-1$
$52-1$

HORAK, HENRY L. (WX-8) $16-14$
$16-15$

HORAN $_{3-57}$ PAUL K. $(\mathrm{H}-10)$

HORNE, JOHN LOUIS $(\mathrm{H}-10)$ $3-25$
$2-26$

HOROWITZ, Y. S.

HORTON, EVERETT H. $(\mathrm{G}-4)$

HOUSEMAN, BARTON L.

HOWARD, J.

HOWELLL, JO ANN (C-3)

HOWELL, ROBERT B. (CTR-2)
$19-1$
$19-6$

HSUE $_{\substack{56-j 8 \\ 56-19}} \mathrm{~S} \cdot(Q-1)$

HUANG, CHAO-YUAN (Q-10) $40-2$
$40-23$
$50-41$
$52-3$

HUANG, CHAO-YUAN (Q-26)

HUDIS, JEROME
$50-91$

HUEBNER, WALTER F. $(T-4)$
$51-37$
$51-31$

$\underset{51-43}{\text { HUEBNER, WALTER F. }(T-4)}$

HUGHES, WILLIAM M. (Q-8) $50-64$
$50-106$

$\underset{53-7\}}{\text { HUIZENGA, }}$ J. R.

HULBERT $55-10$ L. E.

HULET $\frac{53}{53-26}$ K.

HULL, $\underset{5-34}{\text { DONALD E. (CMB-7) }}$

HUNGERFORD, E. V.

HUNN, BRUCE D. $(W X-4)$

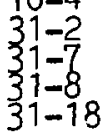

HUNT $\begin{gathered}41-15 \\ 41-105\end{gathered}$

HUPF, $\underset{2-77}{\mathrm{H}}$. $\mathrm{B} \cdot(\mathrm{CNC}-11)$ 
HURLEY, GEORGE F. (CMB-5)

HURSTHOUSE, M. B.

HUTCHINSON, WILLIAM B. (CMB-1) 5-16

HUTSON, RICHARD L. (MP-3) 2-32

HYER, RONALD C. (AP-4)

HYMAN, JAMES M.
$50-67$

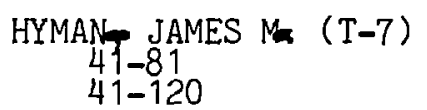

INGRAHAM, JOHN C. (CTR-1)

INOUE, $\underset{47-8}{H}$

$\underset{53-70}{\text { IVERSEN, STEVEN }}(M P-10)$

$\underset{5-20}{\text { JACKSON, DARRYL D. (CMB-1) }}$

$\underset{2-67}{\text { JACKSON }} \underset{\text { DOUGLAS E. }}{(\mathrm{H}-10)}$

$\underset{38-6}{\text { JACKSON, JAMES F. (Q-DO) }}$

JACKSON, JAMES F. (Q-DO) COMP. $26-15$
$38-5$

JACKSON $26-14$ JAMES F . (Q-DO) COMP.

$\underset{53-69}{\text { JACKSON, SYDNEY V. }}$ (P-2)

JACOBSON, ABRAM R. (CTR-2)

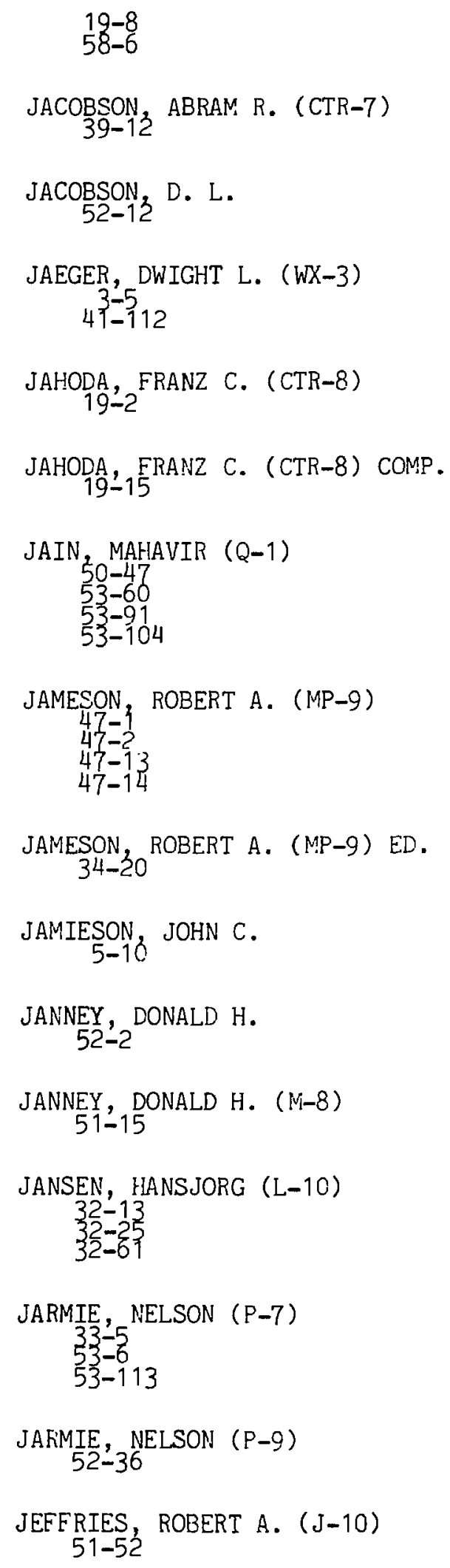




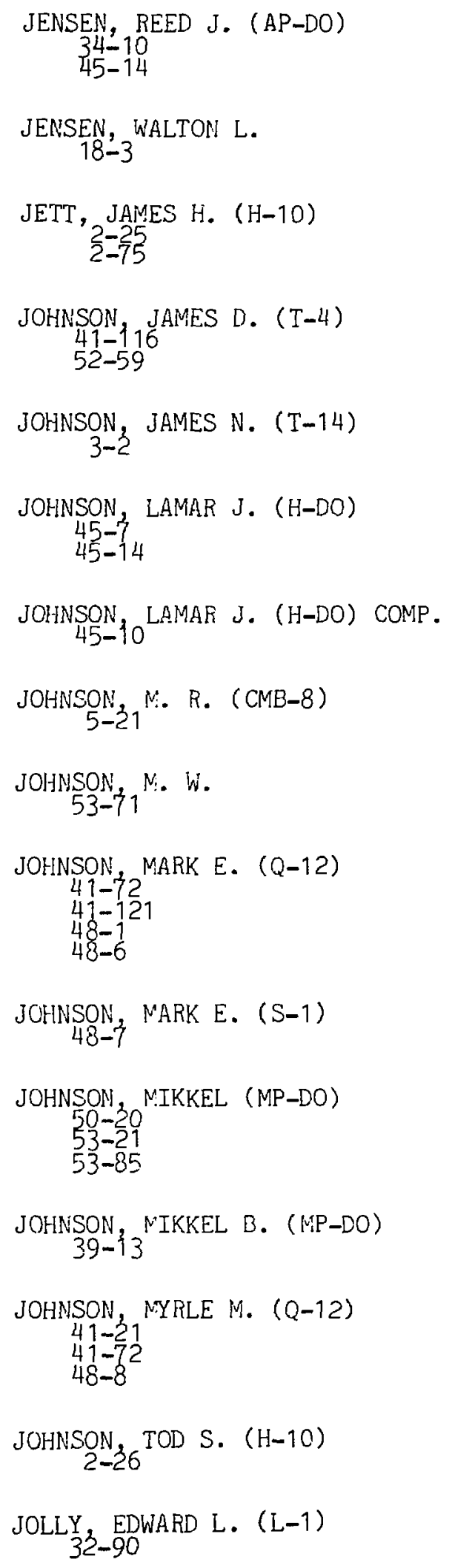

JONES, LARRY A. $(\mathrm{P}-3)$ $19-17$

JONES, LLEWELLYN H. (CNC-4)

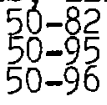

JONES, THOMAS I., JR. (CMB-6)

JONES, WESLEY $M_{15} \cdot 14$ (CME-3)

JOST, $2-17$

JULE ${ }_{47-14}$ WILLARD E.

JURNEY, EDWARD T. (P-DO) $50-49$

$\underset{41-42}{\text { KAHANER }}$ DAVID K. (C-3)

KAHN, LUIS R.<smiles>C1=C[As]2C[As]1C2</smiles>

$50-109$

50-110

$\underset{56-19}{K A I E D A}, \quad(Q-1)$

KARL, ROBERT R. (AP-3)

$34-9$

$50-65$

KARR $\underset{99-6}{\text { HUGH }} \mathrm{J} \cdot$ 
KARR $\underset{19-1}{\text { HUGH }}$ J. (CTR-2)

KATZ $\underset{40-18}{\text { HARRY S. }}$.

KATZ, ${ }_{40-14}^{\text {HARRY }} \mathrm{S}$.

ED.

KAZEK, CHESTER S., JR. (C-5) $41-35$
$41-48$

KEATON, P. W., JR. (E-DO) $27-3$

KEENAN, THOMAS K. (H-7) $45-11$
$45-13$

KEEPIN, G. ROBERT (Q-DO)
$56-30$

KEEPJN, G. ROBERT (R-DO)
$56-17$

$\underset{53-21}{\text { KEISTER }}$ E. D. (NP-DO)

KELLER, CHARLES F. $(J-15)$

$$
\begin{aligned}
& 51-15 \\
& 51-49
\end{aligned}
$$

KELLER, WILLIAM E. (Q-10)

$$
\begin{aligned}
& 11-3 \\
& 11-6 \\
& 11-8 \\
& 15-13
\end{aligned}
$$

KELLER, WILLIAM E. (Q-10) COMP.

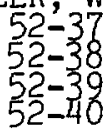

KELLEY, PATRICIA A. (E-DO) COMP. $27-3$

KELLNER, RICHARD G. (C-6)

$$
\begin{aligned}
& 41-54 \\
& 41-91
\end{aligned}
$$

KELLOGG, MARTIN P. (MP-1)

$41-46$
$41-57$
KEMP $\underset{\substack{16-19 \\ 20-8}}{\text { EDWIN L., JR. (CTR-DO) }}$

KENNEDY, R. CRAIG (AP-3)

$$
\begin{aligned}
& 50-51 \\
& 50-96
\end{aligned}
$$

$\underset{50-82}{\text { KENNEDY }}{ }^{R \cdot C R A I G}(A P-4)$

KEPHART, J. E.

KERLEY, GERALD I. (T-4)

$$
32-59
$$

KERN, EDWARD A. (Q-4) $56-1$
$56-13$

KERR, VERNON N. $(\mathrm{H}-11)$

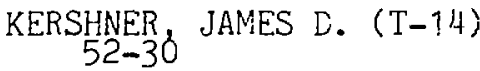

KEYSER, RICHARD CHESTER $(W X-1)$

$\underset{53-100}{\text { KEYWORTH }}$ GEORGE A. $(\mathrm{P}-3)$

KHAPE $\frac{V}{50-85}$

$\underset{2-62}{K I D D E R}, \mathrm{H}$.

KIDMAN, RUSSELL B. (T-2)

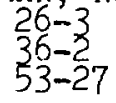

KILLIAN, BARBARA GERMAIN ( $J-9$ ) $18-33$
$45-14$
$52-8$

KIM, KYU C. (AP-4)

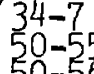

$50-56$

$50-73$
$50-129$

KINCAID, JOHN M. (T-11) 


\section{$52-31$
$52-43$
$52-64$}

KINDEL, JOSEPH M. (L-6) 32-33

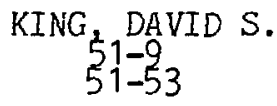

KING $_{50-51}$ JOE DAVID (AP-4)

KINTZ INGER, PAUL R. $(G-6)$

KIRCHER MARY $(L-10)$
$32-24$
$32-54$

KISSANE, RICHARD J. (H-10)

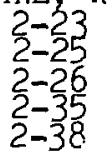

KLARE, KENNETH A. (CTR-8) $19-18$
$20-11$

$20-12$

KLETT $47-20$ JAMES D.

KLIGERMAN, MORTON M. (DIR/ADRT) $2-29$

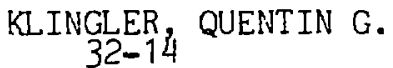

KLINGNER, $\underset{41-114}{C}$. THOMAS $(C-4)$

KNAB, $\underset{5-38}{\text { DARYL }}(\mathrm{H}-8)$

KNAPP 32 CHARLES E. (L-10)

KNAPP, EDWARD A. (AT-DO) $47-23$
$47-28$

KNAPP, EDWARD A. (MP-3) $2-1$
$2-54$
KODAMA, T.

KODIS J JOHN W. $(J-10)$

KOEHLER $2-40$ ANDY $M$.

KOELLE, ALFRED R. $(E-3)$

KOELLE, ALFRED R. (E-5)

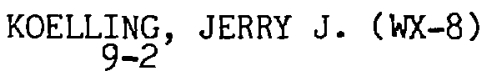

KOENIG, DANIEL R. $(Q-13)$

$26-10$
$41-38$

KOESTEL, ALFRED (Q-6)

KOHLER, DAVID R. (L-4)

KOLLMAN VICTOR H. (H-11)

$2-52$
$50-117$

KOLSTAD, CHARLES D. $(Q-12)$ $18-28$ $31-5$

KOONIN, S. E.

KOSIEWICZ, STANLEY (CMB-1) $45-3$
$45-4$
$45-5$

KOURI $\frac{1}{50}-8.85$

KOZLOWSKI
$53-90$

$\underset{2-33}{\text { KRAEMER, }}$ PAUL M. (H-9)

KRAKOWSKI, ROBERT A. (CTR-DO) 39-7 
KRAKOWSKI, ROBERT A. (CTR-12)

$20-5$
$20=34$
$39-4$

KRAUSHAAR, J. J.

KRAUSSE GEORGE J., III (MP-4) $47-3$
$53-11$

$\underset{19-7}{\text { KRISTAL, RICHARD (CTR-8) }}$

KROGER, PETER M.
$50-93$

KROHN, BURTON J. (T-12) $50-51$
$50-82$

KRUGER, RICHARD (M-8)

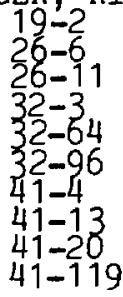

$\underset{40-40}{\text { KRUPKA, MILTON C. (CMB-3) }}$

KUBAS, GREGORY J. (CNC-4) $5-23$

KUCKERTZ

KUMAR, K.

KURNIT, NORMAN A. (AP-2)

$33-14$
$33-16$

KURTENBACH, JOSEPH (SD-5)

$23-1$
$23-2$

KUTAC, KENNETH J. (CTR-4) $20-11$
$20-12$
$\underset{50-93}{K W E I}$ GEORGE H. ( CNC-2)

$\mathrm{KYLE}_{53-90}$ GARY $S .(N P-10)$

KYLE, THOM:AS GAIL (H-5) $30-18$
$32-84$

LA. DELFE, CAROL M. $(\mathrm{G}-5)$

LABAUVE, FAPHAEL J. (T-2)

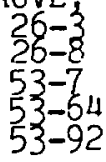

LAERY J JOHN (H-8) $18-22$

LADISH, JOSEPH S. $(\mathrm{L}-1)$
$32-89$
$32-90$

LAHART, M. J. $(M-8)$ L:HART, MARTIN J. (M-8)

$\underset{51-411}{\text { LAMBERT }}$ L. L.

LAMKIN, DAVID E. (Q-6)

LAND $\underset{40-27}{\text { CLETIS } C .}(\mathrm{CMB}-5)$

$\underset{53-26}{\text { LANDRUM }_{3}} \mathrm{~J} . \mathrm{H}$.

LANDT, JEREMY A. (E-DOR)
$23-5$ )
$41-123$

LANE, NEAL $F$.

LANE, $\underset{2-29}{\text { THOMAS }} \mathrm{F} .(\mathrm{WX}-5)$

LANG $\underset{53-90}{P} \dot{P}^{M .}$ 


$$
\begin{aligned}
& \underset{41-19}{\text { LAMGFORD }} \text { JCHN LEE }(h X-3) \\
& \begin{array}{c}
\text { LANGNER } \\
5-34 \\
56-20
\end{array}
\end{aligned}
$$

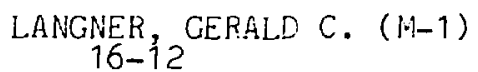

$$
\begin{aligned}
& \text { LAQUER, HENRY L. }(0-26) \\
& \text { LARSEN, EDWARD W. }(\mathrm{T}-1) \\
& \begin{array}{l}
50-25 \\
52-51 \\
52-62
\end{array} \\
& \underset{52-26}{\text { LAPSON, ALVIN R. }} \cdot(L-5) \\
& \underset{40-23}{\text { LASKOWSKI }} \text { R. J. (Q-10) }
\end{aligned}
$$

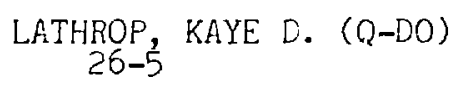

$$
\begin{aligned}
& \text { LAUEF, DONALD J. }(W X-3) \\
& \begin{array}{l}
\text { LAUGHLIN, A. WILLIAM }(G-9) \\
18-32
\end{array} \\
& \text { LAWTON, ROBERT G. (C-3) } \\
& \text { LAYMEY: } 27-14 \text { EWARD R. (PER-DO) } \\
& \begin{array}{l}
\text { LAZARUS } \\
41-25 \\
41-64
\end{array} \\
& \begin{array}{l}
\text { LAZAFUS, FOGER B. }(\mathrm{Q}-6) \\
25-1 \\
25-5
\end{array} \\
& \begin{array}{l}
\text { LAZNZZERA } \\
32-101
\end{array} \\
& \text { LEARY, KEVIN M. (AP-3) } \\
& \begin{array}{l}
32=1 \\
24-11
\end{array} \\
& \text { LEARY }{ }_{34-1}^{\text {KEVIN M. (AP-4) }}
\end{aligned}
$$

$$
\begin{array}{r}
\text { LEBRUN, } \begin{array}{r}
5 \\
53-98 \\
53-103 \\
\text { LEDERER } \\
47-7
\end{array} \text { H. M. (MP-12) }
\end{array}
$$

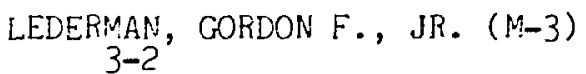$$
\text { LEE, } \underset{53-86}{\text { DAVID M. (MP-3) }}
$$$$
\text { LEE, JAMES T., JR. (AP-1) }
$$$$
\text { LEE, JOHN } C .
$$$$
\text { LEE, KENHETH }
$$$$
\text { LEE, } \underset{58-10}{\text { KENNTH }}(L-6)
$$$$
\text { LEE, PING }(L-4)
$$$$
\begin{aligned}
& \text { LEHMAN, DONALD } R \text {. } \\
& 53-50
\end{aligned}
$$$$
\text { LEHMANN }{ }_{51-26}^{K}
$$

LELAMD, WALLACE T. (L-10)

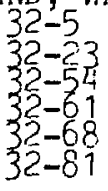

LEMONS, DON S. (P-4)

$$
\begin{aligned}
& 51-4 \\
& 51-13
\end{aligned}
$$$$
\begin{gathered}
\text { LEON }_{50} \text { MELVIN (MP-3) } \\
50-31 \\
50-40
\end{gathered}
$$

LEONARD, ELLEN M. (Q-12) $18-66$

LESTER, JEAN V. 
LEWIS $_{2-60}$

LEWIS, ${ }_{45-10}$ MAXINE $(H-12)$ COMP.

LEWIS RICHARD E. (C-DO-R)

LI, LING-FONG

53-74

LIBERMAN DAVID A.
$50-114$

$\underset{32-14}{\text { LIBERMAN, IRVING }(L-1)}$

LICHTENSTEIN, LAWRENCE $M$.

LIEBENBERG, DONALD H. (Q-10)

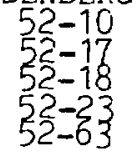

LIEBER, ALBERT $(\mathrm{J}-14)$

$32-67$
$52-24$

LIEBER,

I.IEWER, PAULETTE C.

LIGHT, JOHN C.

$50-9$
$50-86$

$50-86$
$50-105$

$\operatorname{LILES}_{35-7}$ DENNIS $R \cdot(Q-6)$

LILJESTRAND, R. $53-3$

LILJESTRAND, R. (MP-10)

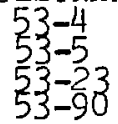

LILLBERG, JOHN W. (CTR-8) $19-14$
$41-82$
$\mathrm{LINL}_{53-\dot{8} 1} \mathrm{D}$.

LINDMAN
$5 \varepsilon-10$

LIRDOUIST, LLOYD O. (MP-7)
$47-29$

LINIDSTRAND, RICHARD $(L-10)$ $32-5$

LINN, GECRGE $\cdots \cdot(G-6)$

LITTLEFIELD, LAFRY $(\mathrm{H}-5)$

LITTLEJOHN, GEORGF. I. $(\mathrm{H}-1)$ $30-6$
$30-20$
$30-2 ?$

LOFA $32-5$

LOHRDING, RONALE Y.. $(G-12)$

LOHRENZ, JOHN

$46-2$
$48-5$
$48-9$

LONDON, JFREY E. (H-4)

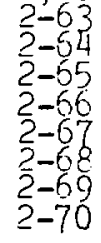

LONDON, FOEERT E. ( $\mathrm{CNC}-4)$

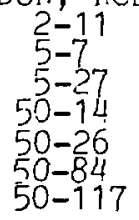

LONDON, $2-12$ RCBERT E. (CNC-4)

LONG MARSHALL 
LOONEY, LARRY D. (J-14)

$16-8$
$16-9$
$52-24$

LOOSE, VERNE W. (S-2)

$17-4$
$26-7$

LOREE, THOMAS R. (AP-2)

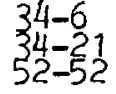

LOS ALAMOS SCIENTIFIC LABORATORY $29-2$
$41-32$

$\underset{53-26}{\text { LOUGHEED, }}$ R. W.

LOVE, JOHN W. (E-5)

LOVHOIDEN, G.
$53-80$

LOW'E, ARTHUR T. (L-7) $32-40$
$32-75$
$32-76$

LOWE, $_{56-25}$ VICTOR W., JR. $(Q-12)$

LOWRY, PHILIP L. (H-5) $30-7$

LUCAS,

LUCK $_{3}$ LARRY B. $(Q-7)$
$38-7$
$38-9$

LUCKSTEAD, STEVEN $C$.
$53-28$

LUDEMANN, C. A. $53-32$
$53-87$

LUNDBERG, LYNN B. (Q-13)

LUNDY, ARVID S. (E-5)

$$
\begin{aligned}
& 33-2 \\
& 41-20
\end{aligned}
$$

LUNSFORD, JAMES S. (E-3)

LUNSFORD, JOHN L. (CMB-8)

LUTH, W. $_{45} \mathrm{C}$.

LUTZ $_{52-24} S_{2}$

LYMAN, $_{2-40} \mathrm{~J}$.

LYMAN, JOHN L. (AP-3)

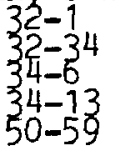

$\underset{34-1}{\text { LYMAN, JOHN L. }(A P-4)}$

$\mathrm{LYNN}_{37-16}$ DAYID $(E-4)$

$\mathrm{LYNN}_{50-28} \mathrm{~K} \cdot \mathrm{G}$.

LYONS, PETER E. $(J-14)$

$16-9$

$16-10$
$52-24$

MAAS, LYNN D. (C-6)

$41-51$
$41-52$
$41-56$

MAC DONALD, JAMES L. $(\mathrm{T}-1)$ 39-17

MAC FARLANE, ROBERT E. (T-2)

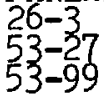

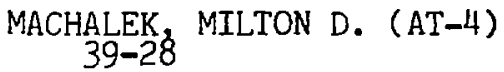

MACHALEK, MILTON D. (CTR-2) TRANSLATOR 
$37-6$

$\underset{39-15}{\text { MACHALEK }}$ MILTON D. (CTR-7)

MACK JOSEPH M., JR. (J-15) $50-60$

$52-35$
$58-3$

MACKLIN $\frac{53-45}{\text { R. L. }}$

MACRAE, KENNETH I. (T-8)

$54-10$
$54-11$

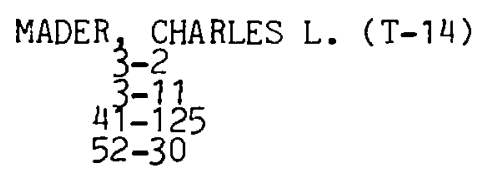

MADER 3 CHARLES L. $(T-14)$

$3-2$

$4{ }^{3}-125$

52-30

MADLAND, DAVID G. (T-2)

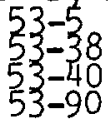

MAECK $_{18-26}^{\text {WILLIAM J. }}$

$\underset{50-6\}}{\text { MAGGIORE, CARL J. }}(E-10)$

$\mathrm{MAH}, \underset{32-62}{\operatorname{RICHARD}}(\mathrm{CMB}-6)$

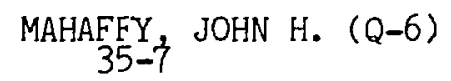

MAIER, WILLIAM B., II (AP-4)

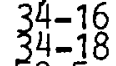

$50-5$
$50-34$

$50-35$
$50-97$

$\underset{50-33}{\text { MAIER, WILLIAM B. , II (L-3) }}$

MALANIFY
$50-83$ JOHN J. $(P-11)$

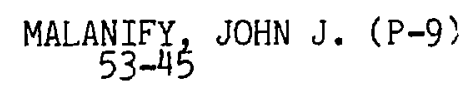

MALBROUGH, DONALD J.

$53-32$
$53-86$

MALCOM, JACK E. $(E-1)$

MALENFANT, RICHARD E. (Q-DO)

MALEY MARTIN P. $(Q-10)$

MALEY, MARTIN P. $(Q-10)$ COMP. $52-33$
$52-34$

MALIK $\underset{52-70}{\text { JOHN S. (J-DOT) }}$

MALTRUD, HERMAN R. (L-7)

$\underset{53-55^{2}}{\text { MANASSAH }}$ T.

MANCA, JOSEPH J. (AT-1)
$47-23$
$47-28$

MANCA, JOSEPH J. (P-5)

MANCA VARLERIE M. (ISD-4) TRAINSLATOR
MO 29

MANN $_{20-26}^{\text {LAWRY }}$ N. (CTR-10)

$\underset{32-14}{\text { MANNING }}$ JOHN P.

MARKS, THOMAS

$53-32$
$53-86$
$53-87$

MARSH, SINCLAIR F. (CME-1)
$5-1$
$5-4$
$5-30$

MARSHALL, JOHN, JR. (CTR-DO)
$20-13$

MARTELL, CALVIN J. (CMB-1) 


$$
\text { MARTIN, A. DON }(E-2)
$$

MAFTIN, JOHN C. $(\mathrm{H}-10)$ $2-5$
$20-26$
$20-116$

MARTIN $2-6$ JOHN C. (LS-2)

MARTIN, FICHARD A. (WX-8)

ViARTIN, ROBERT W. $(\mathrm{H}-1)$

$\underset{3-14}{\text { MARTINEZ }}$ CARLOS A. (WX-3)

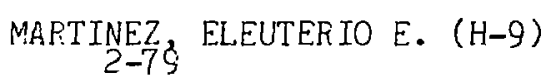

MARTZ $\}_{-6}$ IIARRY F., JR.

MARTZ
$38-3$
$38-4$
$38-4$

$\underset{47-29}{\text { MARUSAK, ALEX L. }}(C-3)$

MASON, CAROLINE F. V. (CMB-3) $5-12$

MAST, PETER K. (Q-7) $36-5$
$38-8$

MATIS ${ }_{53-74}^{H}$ S.

MATTHIAS, B. T.
$40-20$
$40-46$

MATUSKA, WALTER $(\mathrm{J}-15)$

$$
\begin{gathered}
1-1 \\
51-15
\end{gathered}
$$

WATWIYOFF, NICHOLAS A. (CNC-4)

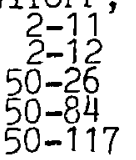

MAURER, W. C.

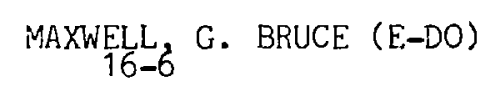

MAXWELL, JAMES C. (G-5)
$18-29$
$18-30$

$\underset{32-77}{\text { MAYER, ANTON (CMB-6) }}$

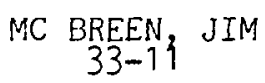

ED.

MC CONVILLE, JOHN T.

MC $\underset{27-16}{\text { CORMICK }}$, J. BYRON $(E-4)$

MC CORMICK, J. BYRON (E-4) ED.
$27-10$
$33-11$

MC CREARY, WILLIAM J. (CMB-6)
$32-6$
$57-3$

MC DANIELS, DAVID K.

$53-3$
$53-5$

MC DONALD, THOMAS E. (E-4)
$16-4$
$31-18$

MC DOWELL, ROBIN S. (AP-4)
$32-47$
$50-51$
$50-52$

MC FARLAND, JAMES $W$.
$48-6$
$48-9$

MC FARLAND, LARRY C. (ISD-6) COMP.

MC FARLAND, ROBERT D. (Q-11)

$31-12$
$31=13$
$31-14$

MC GETCHIN, THOMAS R. (G-6) $24-12$
$51-27$ 
MC KAY, MICHAEL D. (Q-12)

$$
\begin{aligned}
& 26-1 \\
& 44-1 \\
& 44-3
\end{aligned}
$$

MC KAY, MICHAEL D. (S-1)

$$
27-18
$$

MC KENNA, KENNETH F. (CTR-3)

MC LAUGHLIN, DONALD R. (CNC-2)

MC LELLAN, EDWARD 3. (L-9)

MC LEOD, M. J. (CMB-1)

MC QUEEN, JOHN H.

MEDINA, EVITA G. $(Q-1)$

$$
\begin{aligned}
& 35-6 \\
& 56-19
\end{aligned}
$$

MEEK, ${ }_{5-46} W$.

MEIER, KARL L. $(P-14)$

$$
\begin{aligned}
& 39-5 \\
& 52-32
\end{aligned}
$$

MEIKLE, W. PETER S.

$$
51-48
$$

MEIRON, DANIEL I.

$$
41 \cdot-30
$$

MELSA J JAMES L.

MELTON, JIMMY G. (CTR-11)

$$
\begin{aligned}
& 20-7 \\
& 20-9
\end{aligned}
$$

MENDELSOHN, J.

$\underset{52-29}{\operatorname{MENIKOFF}}$ RALPH (T-DOT)

MENIKOFF, RALPH (T-14)
MENLOVE, HOWARD O. (Q-1)

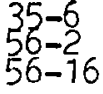

$\underset{44-5}{\text { MENLOVE, HOWARD }}$ O. (Q-1) COMP.

MERLAN, STEPHEN J. (DIR OFF)
$27-13$

MERSON, JEAN L. $(W X-8)$

MERSON, THOMAS J. $(w X-4)$

METROPOLIS, NICHCLAS C. (T-7)

$47-80$

$41-83$

MEYER, RICHARD A.

$\underset{51-3}{\text { MICHAJD, GEORGES }}$

MIERA, FELIX R., JR. $(H-12)$
$18-11$
$18-41$

MIERA, FELIX R., JR. $(H-8)$

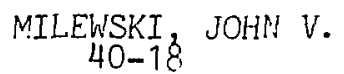

$\underset{40-14}{M I L E W S K}$ JOHN $V$.

ED.

MILEWSKI J0-48 JOHN V. (L-7)

MILLER, GUTHRIE (CTR-3)

$19-10$
$19-12$
$20-11$
$20-12$
$30-19$

MILLER, JOHN R. (L-7)

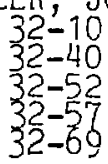


MILLER, L. W. $(J-15)$

$1-1$
$52-35$

MILLER, WARREN F., JR. (T-1)

$26-22$
$26-24$

$26-25$

$53-478$

MILLER, WARREN F., JR. (T-1) COMP.
$26-23$

MILLS, RENE SCOTT (MP-9)

MILLS, ROBERT $(Q-10)$

MILLS, ROBERT L. (CMB-5)
$5=-10$

MILLS, ROBERT L. $(Q-10)$ $52-18$

$52-23$
$52-63$

MINOR, MICHAEL M. (P-2) $4 \begin{aligned} & 5-6 \\ & 4-57\end{aligned}$

MIRMIRANI, MAJDEDIN
$2-59$

MISCHKE, RICHARD E. (MP-4)

$\underset{32-38}{\text { MITCHELL, KENNETH B. }(L-1)}$

MIX, ${ }_{32}=36$ PAUL, JR.

MJOLSNESS, RAYMOND C. $(T-3)$
$52-29$
$52-61$

MOINESTER, M. A. (MP-4)

MOLLER, PETER $(T-9)$

MOIASH, ELLIS A.
$48-6$
$48-7$
$48-9$

MONTGOMERY, DAVID
$39-22$

MONTGOMERY, MICHAEL D. $(L-1)$ $32=89$

MOORE, ${ }_{53-84}$ FRED

MOORE $\frac{53-70}{C}$ FRED (MP-10)

MOORE, GLENN H.
$27-18$

MOORE, MICHAEL S. $(P-3)$

MOORE ${ }_{3}$ STANLEY W. $(\mathrm{Q}-11)$

MORALES, RAUL $(\mathrm{H}-5)$
$30-5$
$30-10$

MORE $_{32-53}$ RICHARD

MORGAN, LEON O. (CNC-4) $5-26$
$50-79$

MORGAN, TERRANCE L. (G-5)

MORRIS, CHRISTOPHER L. (MP-10)

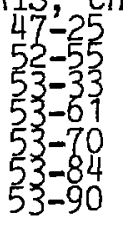

MORRIS, DONALD W. $(S-2)$

MORRIS, GLENN E. (Q-12)

MORRIS $\frac{17}{17}$ GLENN E. (S-2) 
MORRIS $\frac{\text { ROGER A. }}{41-20}(M-1)$

MORRIS, WAYNE A. (G-5)

$18-58$
$18-62$

MORRIS, WAYNE A. (J-5) $18-18$
$18-52$

MORRISON, JOHN F. $(\mathrm{C}-11)$ COMP.

MORRISON, MICHAEL A. (T-12) $50-19$

$51-12$

MORTENSEN, JEANNETTE J. (G-DOT) 24-3

$\underset{53-100}{\operatorname{MOSES}} \underset{30 H N}{ }$ D. $(P-3)$

MOSS, CALVIN E. (Q-2) 53-35

$\underset{50-52}{\text { MOULTON, }}$ PETER F.

MOUSSA, PIERRE

52-60

$\underset{5-28}{\text { MUELLER }} \underset{3 A R B A R A}{\text { A }} \cdot($ CMB-3)

MUELLER, D. W.

MULLANEY, PAUL F. (H-10)

$2-5$
$2-6$
$2-25$

MULLER, MARTIN (WX-4)

$17-1$
$20-4$

MUNROE $3:-4$ JAMES L. $(L-10)$

MURPHY $\underset{24-13}{\text { HUGH D. }}(\mathrm{G}-3)$

MURPHY, HUGH D. (G-4)

\section{$21-3$
$24-11$
$24-14$}

MURRAY, HUGH S. (E-4) $31=11$

$\underset{18-66}{\text { MUTSCHLECNER, J. PAUL }}$

MYERS, THOMAS A. $(\mathrm{CNC}-11)$

NACHAMKIN, JACK (E-DOR)
$27-16$,

NAGLE $\frac{\text { DARRAGH E. }}{50119}$ (MP-DO) LOMP.

NANN $50 \mathrm{H}-\mathrm{i} 18$

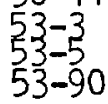

$\underset{18-5}{\text { NASHNER, }}$

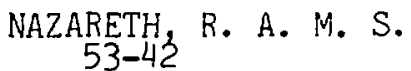

NEE, $\frac{T \cdot W}{50-80}$

NEEPER, DONALD A. $(\mathrm{Q}-11)$ COMP.

NELSON, $M_{1}$. A.

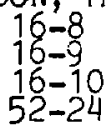

NERESON
$52-72$

NERESON, NORRIS G. (AP-4)

$$
\begin{aligned}
& 50-37 \\
& 50-51 \\
& 50-52
\end{aligned}
$$

NEUDECKER, JOSEPH W. (WX-8)

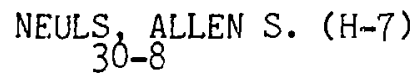


$\underset{\substack{19-3 \\ \text { NEWBERGER, BARRY S. }}}{(\mathrm{T}-15)}$

NEWE IELD
$32-29$
$40-17$
$40-17$

NEWKIRK, LAWRENCE R. ( CMB-3) $40-16$
$40-39$

NEWMAN, MICHAEL J. (T-DOT)

NEWNAM, BRIAN E. (AP-2)

$$
\begin{aligned}
& 32-20 \\
& 32-21
\end{aligned}
$$

NEWSOM, $C$. $53-60$
$53-104$

$\underset{41-109}{\text { NICHOLS, EASIL. }(T-7)}$

NICHOLS, BILL D. $(T-3)$

NICHOLSON, NICHOJAS $(0-2)$
$56-12$

NICKEL, BERNIE G.

NICKELL, J. DONALD, JR. (E-5)
$2 \begin{aligned} & 3 \\ & 4\end{aligned}-5$
-68

NICKOLS, JOYCE W. $(\mathrm{H}-5)$
$30-9$

$\underset{39-11}{\text { NIELSON, CLAIR W. (CTR-10) }}$

NIELSON, CLAIR $W \cdot(L-6)$
$41-131$

NIETO, MICHAEL MARTIN (T-8)

$$
\begin{aligned}
& 52-19 \\
& 54-4
\end{aligned}
$$

NIX, JAMES R. (T-9)

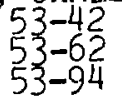

NOEL, BRUCE W. $(\mathrm{J}-8)$

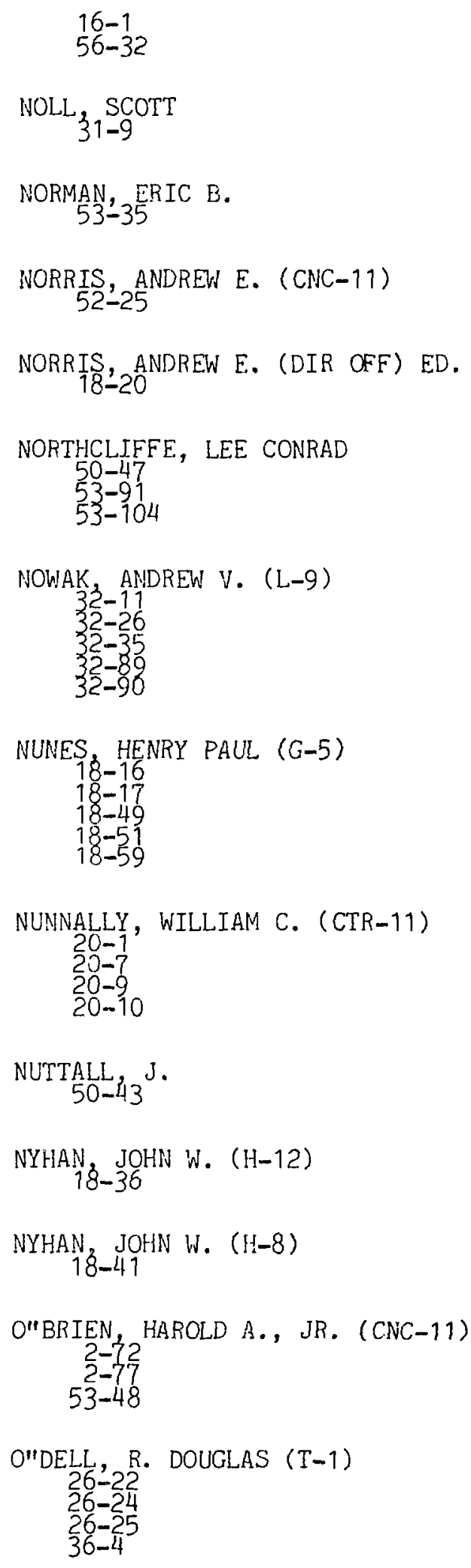


O"DELL, R. R. DOUGLAS (T-1) COMP.

OAKES, WILLIAM R., JR. (WX-3)

OBST $_{53-70}$ ANDREW $(M P-10)$

ODETTE, G. R.

OGLE $_{50-61}$ WILLIAM (WPC- 2 )

OHLSEN, GERALD G. (P-7) $53-6113$

OHLSEN, GERALD G. (P-9)

OKA, MELVIN S. $(H-10)$

$$
\begin{aligned}
& 2-13 \\
& 2-35 \\
& 2-50
\end{aligned}
$$

OLDENBORG, RICHARD C. (AP-3)

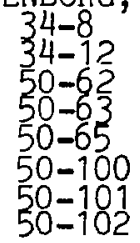

OLDENBORG, RICHARD C. (AP03) 34-9

OLINGER 34 BARTON W. (M-6)

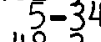

$52-30$

OLMER 5 - C.

OLSEN, CLAYTON E. (G-5)

OLSEN, JULIA D. (CMB-8)

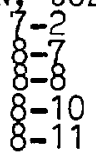

OLSEN, KENNETH H. (G-2)
$18-71$

ONEDA
$53-2 \dot{9}$
$54-12$

ONSTOTT, EDWARD I. (CMB-8)

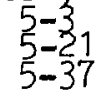

OOTHOUDT, M. A.
$53-3$.
$53-5$
$53-90$

ORBESEN STUART D. (P-7)

53-81

ORBESEN, STUART D. (P-9)

ORLICKY, DAVID J. (H-10)

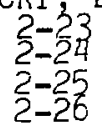

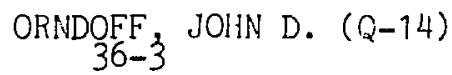

$\mathrm{ORTH}_{50-91}$ CHARLES J. (CNC-11)

ORTIZ, LRONRENCE W. $(\mathrm{H}-5)$

$\mathrm{OSBORNE}_{2-62}$ W. Z.

OSETEK, DANIEL $J$.

OSETEK, DANIEL J. (H-5)

OSTER, GEORGE E.

OTT, DOHALD G. $(\mathrm{H}-11)$

OTT, $\underset{53-44}{\text { MARTIN ADOLF (CNC-11) }}$

OVERTON, WILLIAM C., JR. (Q-10) 


\section{$52-49$
$52-50$}

$\underset{18-68}{\text { OWENS JAMES } \mathrm{W} .(\mathrm{H}-8)}$

PACIOTTI, MICHAEL A. (MP-3)

PACK RUSSELL T. (T-12)

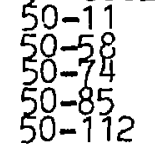

PAIK $_{53-99} \mathrm{~N}$.

PALMER, IAN D.
$51-5$
$51-30$

PALMER, IAN D. $(\mathrm{P}-4)$

PALMER, RONALD G. $(Q-12)$
$18-28$

PARKER, FREDERICK (Q-7)

PARKER, GREGORY A.
$50-112$

PARKER, GREGORY A. (T-12) $50-58$

PARKER, JACK L. (Q-1)

PARKER, JOSEPH R. (E-5)

PARKIN, DON M. (CMB-8) $40-9$
$50-28$
$53-20$

PARKINSON, WILLIAM J. (WX-4)

PARTER, S. V.

PASCHMANN, GOTZ
$51-26$

PATTERSON,
$50-48$ C. W. (T-7)

PAULETTA, G.
$53-3$
$53-5$

PAXTON, HUGH C. $(Q-14)$

PAXTON, HUGH C. (Q-14) COMP.

PAYNE, JAMES B. (WX-8)

PAYNE, ROBERT J. $(\mathrm{H}-6)$

$$
41-78
$$

PEARLSTEIN, L. D. $58-5$
$58-11$

PENDERGRASS, JOHN H. (L-5)

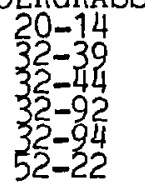

PENDLEY $18-28$ ROBERT (Q-12)

PENTECOST, BENLEE A. (WX-3)

PERELSON, ALAN S. $(T-10)$

$$
\begin{aligned}
& 2-4 \\
& 2-47 \\
& 2-47 \\
& 2-56 \\
& 2-59 \\
& 2-80 \\
& 2-81 \\
& 2-81
\end{aligned}
$$

PERKINS $_{32-55}$ ROGER B. (L-DO)

$\underset{2-17}{\text { PERNICK, }}$ B. P.

PERREAULT,
$2-60$ 


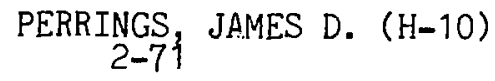

${ }_{53-87}^{\text {PERROUD }}$ J. P.

PERRY, DENNIS G. (MP-1) $41-68$
$53-71$

PERRY, DENNIS G. (MP-1)

PERSON, W. B. (AP-4)

PERSON, WILLIS B. $(A P-2)$
$50-5$

PERSON, WILLIS B. (AP-4)

PERSON, WILLIS B. (T-12) $50-730$
$50-129$

PETERS, RICHARD J. ( $\mathrm{H}-8)$

PETERSEN, DONALD F. (H-DOT) COMP.

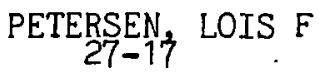

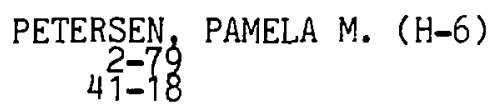

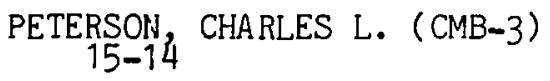

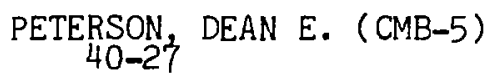

PETERSON, EUGENE J. (CMB-8)

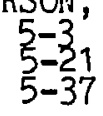

PETERSON, R. J.

$53-16$
$53-84$

PETERSON, ROBERT $\mathrm{W} \cdot(\mathrm{J}-14)$ $\underset{3-3}{\text { PETERSON, SUZANHE } W .(W X-2)}$

PETSCHEK, ALBERT G. $16-27$
$32-71$

PETT $\frac{T T T}{24-1}$ ROLAND A. $(G-4)$

PHILIS, $\underset{53-39}{C}$

PHILLIPS, $2-62$ G. C.

$\underset{37-1}{\text { PHILLIPS, JOHN R. (CMB-1) }}$

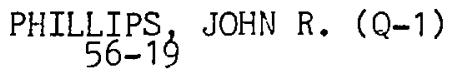

PHILLIPS, RICHARD
46-2

PHIPPS, CLAUDE R., JR. (L-9) $32-16$
$32-70$
$32-93$
$39-10$

$\mathrm{PIGG}_{56-32^{\mathrm{J}}} \mathrm{L}$

PILTCH, MARTIN S. (AP-2)

PIMBLEY $\underset{41-109}{\text { GEORGE H. (T-DOT) }}$

PINDZOLA, MICHAEL S.
$52-36$

PINES, DAVID
$52-53$
$52-54$

PLASSMANN, E. H. $(W X-5)$
$56-26$

PLASSMANN, EUGENE A. (Q-14)

$\underset{50-72}{\text { PLUMMER }}$ 
POGNA ${ }_{56-26}$ ROBERT (DIR-SEC)

POLLOCK ${ }_{32-85}$ CLIFFORD R.

$\underset{32-50}{\text { POLLOCK }}$ CLIFFORD R. (L-9)

POLZER, WILFRED L. (H-12)

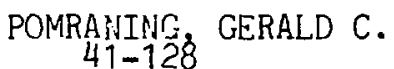

$\underset{51-2.8}{\text { PONGRATZ }}$ MORRIS B. $(J-10)$

POORE, RAY V. $(P-9)$

PORILE, NORBERT T. $53-34$

POTTER, JARED MICHAEL

POTTER, JERRY M. (MP-1)

POTTER, ROBERT M. $(\mathrm{G}-3)$

PRATT $\frac{53-5}{3}$ JHN C. (MP-10)

PRATT $\underset{53-90}{\text { JOHN C. }(Q-2)}$

PREEDOM, BARRY M.

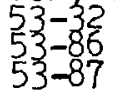

PRESTON, RICHARD K.

PRESTON, RICHARD K. $(T-12)$
$50-122$

PRICE, BRANDON J. $(\mathrm{H}-10)$ $20-52$
$50-116$

PRINE $_{2-21}$ JAMES R. ( $\left.\mathrm{H}-4\right)$
$2-51$
$2-67$

PRUPPACHER, HANS R.
$47-20$

PUBLIC INFCRMATION OFFICE

PURTYMUN, WILLIAM D. (H-8)

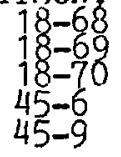

PYUN $_{35-9}$ JUNGJO (Q-6)

QUALLS, ${ }_{54-8}$ CLIFFORD (T-7)

QUIGLEY
$50-15$
$50-64$
$50-106$

$\underset{2}{\text { QUINN }-11}$ WARREN E. (CTR-DO)

QUINN, WARREN E. (CTR-3)
$20-12$

$\underset{52-69}{\text { RABIE, RONALD L. }}(W X-7)$

RADZIEMSKI, LEON J., JR. (AP-4) $50-51$
$58-3$

RAGAN, CHARLES E. $(P-3)$

RAJU, MUDUNDI R. $(\mathrm{H}-10)$

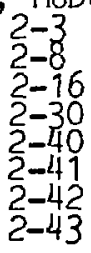

RAMAN, S.

RAMBERG $_{41-121}$ JOHN 


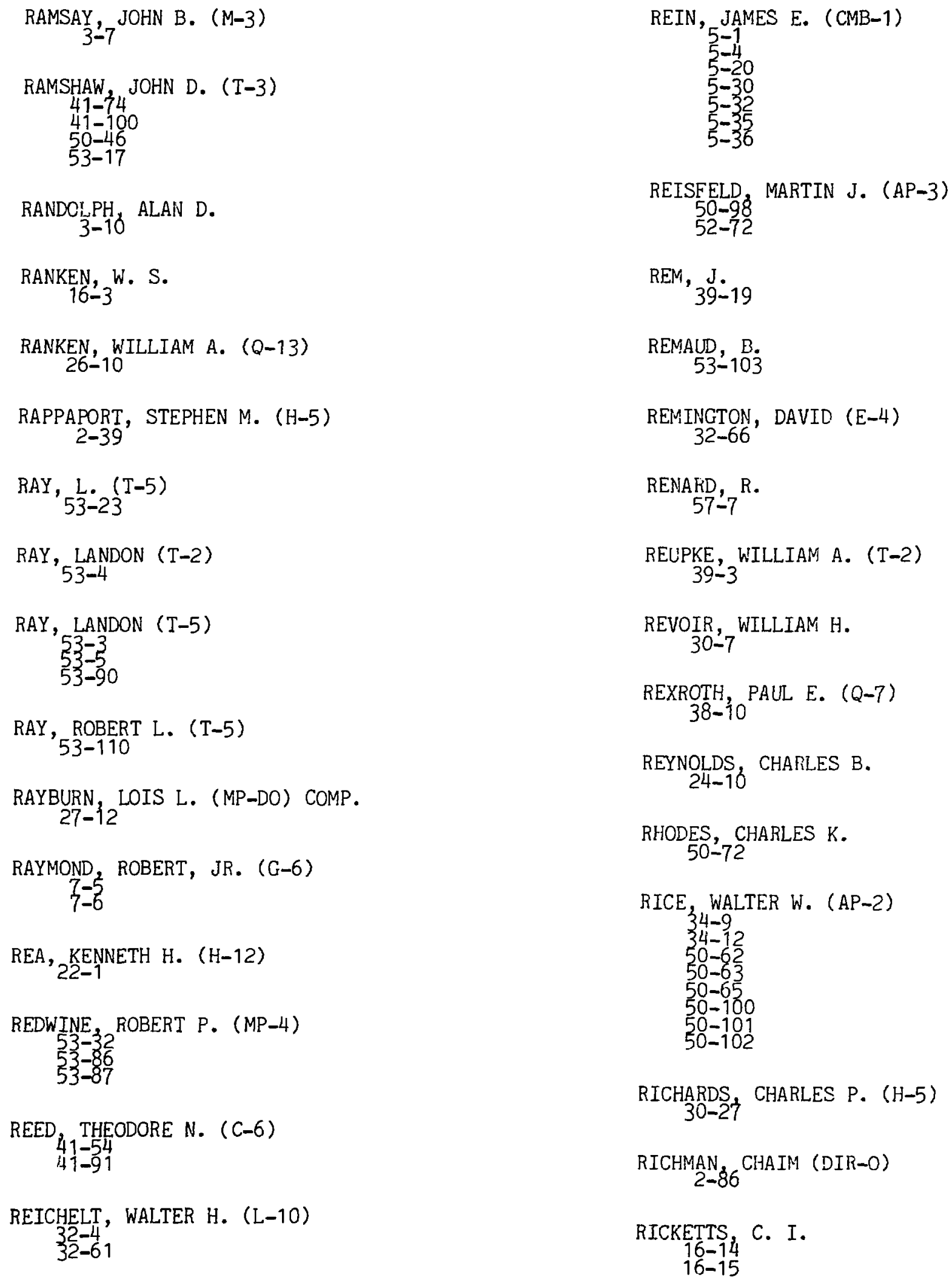




$$
\text { RIDGE, R3-90 J. }
$$$$
\text { RIDGELEY, A. }
$$

RIEPE, KENNETH B. (L-10) $32-5$
$32-24$

RILEY, $P$. J.
$50-47$
$53-60$
$53-91$
$53-104$

RILEY STEPHEN J.

RINK $\underset{41-108}{\text { RAYMOND }(Q-12)}$

RINKER, GEORGE A., JR. $(L-6)$

$$
\text { RISTINEN, R. A. }
$$

RIVARD, WILLIAM C. (T-3) $35-3$
$41-86$

RIVERA, THOMAS $(w X-3)$

RNO, JUNG S.

$\mathrm{ROACH}_{31-9}$ JAMES F. (Q-12)

$\underset{51-52}{\mathrm{ROACH}}$ WILLIAM H. $(\mathrm{J}-12)$

ROBB, $W_{5}$. DEREK (T-4)

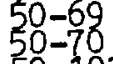

$50-103$

ROBERTSON, JAMES B. $(H-10)$

$$
\begin{array}{r}
2-30 \\
2-41 \\
2-42
\end{array}
$$

ROBICHAUD, R. E.

$$
\begin{aligned}
& \underset{34-10}{\text { ROBINSON, }} \text { C. PAUL (AP-DO) } \\
& \text { ROEINSON, JOEL H. (E-5) } \\
& 41-33 \\
& \text { RODERICK, NORMAN F. } \\
& 32-51 \\
& \text { RODGERS, JOHN C. (H-12) } \\
& 30-24 \\
& \text { RODRIGUEZ, JOE F. (E-1) } \\
& 2-86 \\
& \text { ROEDER, DENNIS L. (MP-13) } \\
& 2-29 \\
& \text { ROENSCH, F. R. (CMB-1) } \\
& 5-1 \\
& 5-4 \\
& 5-20
\end{aligned}
$$

ROFER-DE POORTER, CHERYL K. (AP-3) $50-89$

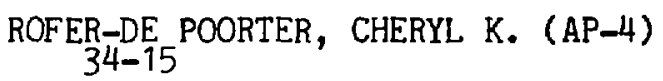

ROGERS, JOHN D., JR. (CTR-9) COMP. 20-25

ROGERS, JOHN D., JR. (Q-DO) $11-4$
$15-9$

ROGERS, JOHN D., JR. (Q-DO) COMP.

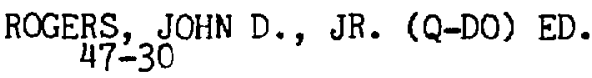

ROGERS, MARGARET ANNE (H-8)

ROHIR $_{40-5}$ DANA L. (CMB-5)

ROMERO, ANGELA $(\mathrm{H}-10)$

ROMERO, LEONARD L. $(\mathrm{H}-1)$

ROMERO, PABLO O. (H-1) 
RONQUILLO, ELIZABETH A. (S-DO) COMP. 27-19

$\operatorname{ROOD}_{52-59}$ GERALD T. $(T-4)$

ROOF, RAYMOND BRADLEY, JR. (CMB-5) $40-27$

$40-32$

$\operatorname{ROSCHKE}_{31-2}$ MARK A. $(W X-4)$

ROSEN, LOUIS (MP-DO) $3-62$
$47-24$

ROSENBAUER, $\mathrm{H}$. $51-26$

ROTA GIAN-CARLO
$41-80$
$41-83$

ROTBARD ${ }_{53}$ G.

$53-103$

ROWLEY, JOHN C. (G-DOT)

ROYBAL, ELIZA U. (MP-9) $47-1$
$47-2$

RUMINER, $3-12$ JOHN J. (WX-3)

RUMINER $\underset{41-31}{4}$ JOHN J. (WX-3) COMP.

RUPPEL, ${ }_{52}-6$ ANS M. $(T-3)$

RUSSELL ${ }_{47-\{1}$ GARY J. (P-11)

RUTZ $\underset{40-13}{\text { HEIDI L. (CMB-6) }}$

RYAN, ROBERT R. (CNC-4) $5-23$
$5-40$
S-2 GROUP

SAFEGUARDS GROUPS 56-31

$\mathrm{SAITO}_{47-9}$ NORIO

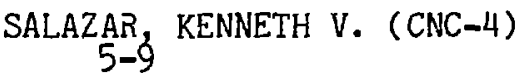

SALTMARSH, M. J.

SALZMAN, GARY C. $(\mathrm{H}-10)$

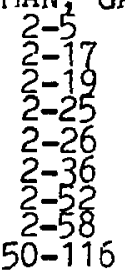

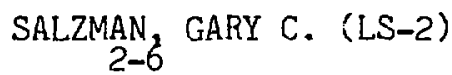

SAMPSON ${ }_{53-93}$ THOMAS E. (Q-2)

SANDENAW, THOMAS A. (CMB-8) $40-11$

$40-47$

SANDERS, WILLIAM MORT ( $\mathrm{H}-6)$

$$
\begin{aligned}
& \text { 2-79 } \\
& 41-18
\end{aligned}
$$

$\underset{50-67}{\text { SANDERSON, JAMES G., JR. (C-8) }}$

SANDERSON, JEFFREY J. (CTR-6)
$58-1$

SANDFORD, MAXWELL T. $(\mathrm{J}-10)$

$$
\begin{aligned}
& 51-1 \\
& 52-1
\end{aligned}
$$

SANDFORD, MAXWELL T. ( $\mathrm{J}-9$ )

$18-23$

$\begin{array}{rl}3 & -1 \\ 51-45 & 10\end{array}$ 
SANDSTROM, DONALD J. (CMB-6) $40-37$

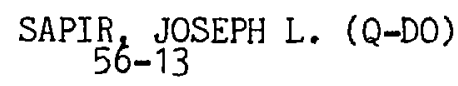

SAPIR JOSEPH L. (Q-DO) COMP. $56-21$
$56-22$

SAUNDERS, GEORGE C. $(\mathrm{H}-10)$ $2-24$
$2-79$

$\underset{32-4}{\operatorname{SAXMAN}}, A \cdot C \cdot(L-10)$

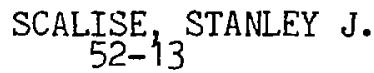

SCANNAPIECO, ANTHONY J. (L-6)

$\underset{47-29}{\text { SCARBROUGH, EDWARD C. (MP-7) }}$

SCHELL, DONALD H. (CMB-6)

SCHELONKA, EDWARD P. (Q-4)

SCHENTER, R. E.

SCHERMER, ROBERT I. (Q-10)

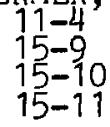

$\underset{5-10}{\text { SCHIFERL, DAVID }(Q-10)}$

SCHILLACI, MARIO E. (MP-3)

SCHMELZEACH, PIERRE

$$
\begin{aligned}
& 53-6 \\
& 53-96
\end{aligned}
$$

SCHMIDT $\frac{52-23}{\text { LEROY C. }}$ (SD-5)

SCHMIDT, P. H.

$$
\begin{aligned}
& 40-2 \\
& 50-41
\end{aligned}
$$

SCHMITTROTH, F.

SCHNEIDER, BARRY (T-12) $50-30$
$50-104$

SCHNEIDER, J. DAVID $(P-14)$

SCHOENMACKERS, RUDI (P-9) 53-112

SCHCTT, GARRY L. (G-7) COMP. 48-10 $48-11$

$\underset{52-7 \delta}{\text { SCHRANDT }}$ ROBERT G. (TD-6)

SCHRODER, W. U. 53-71

$\underset{30-76}{\text { SCHULTE, HARRY F. }} \cdot(\mathrm{H}-5)$

SCHULZE, WILLIAM

SCHUSS, ZEEV

SCHUSTER, BURTON G. $(\mathrm{H}-5)$ $30-18$
$30-31$
$32-84$

SCHWENK, FRANCIS C. $25-3$

SCHWENN, HAROLD R. $(P-3)$

SCKOPKE
$51-34$
$51-33$

$\operatorname{SCOTT} \frac{T}{4}-8 . T$.

SCOTT ${ }_{50-80}^{J} F$.

SCOTT $38-8$ JAMES H. (Q-7) 
SCOTT, PETER B. (AP-2) $33-102$

$52-52$

SCOTT ${ }_{50-42}^{T} A$.

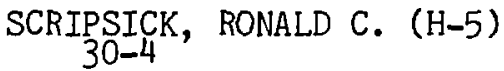

SCULLY, MARLAN 0.

SCULLY, MARLAN O. (L-DO)

$\underset{30-17}{\text { SEAMONS, MAX }}(E-5)$

SECKER, PHILLIP A., JR. (Q-5) $25-1$

SEDLACEK, WILLIAM A. ( CNC-11) $18-15$
$33-13$

SEED THOMAS J. (T-1)

53-108

SEGEL 2 LEE A.

SEGEL, $\underset{53-33}{R}$.

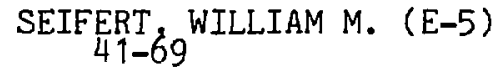

SEKI, RYOICHI (MP-3)

$\operatorname{SERCELY}_{53-16} \mathrm{R} \cdot \mathrm{R}$.

SETH, KAMAL K,

$53-3$

$53-90$

SETH, KAMAL K. (MP-10) 53-70

SGRO $\underset{39-1}{\text { ANTHONY G. }} \cdot(\mathrm{CTR}-6)$

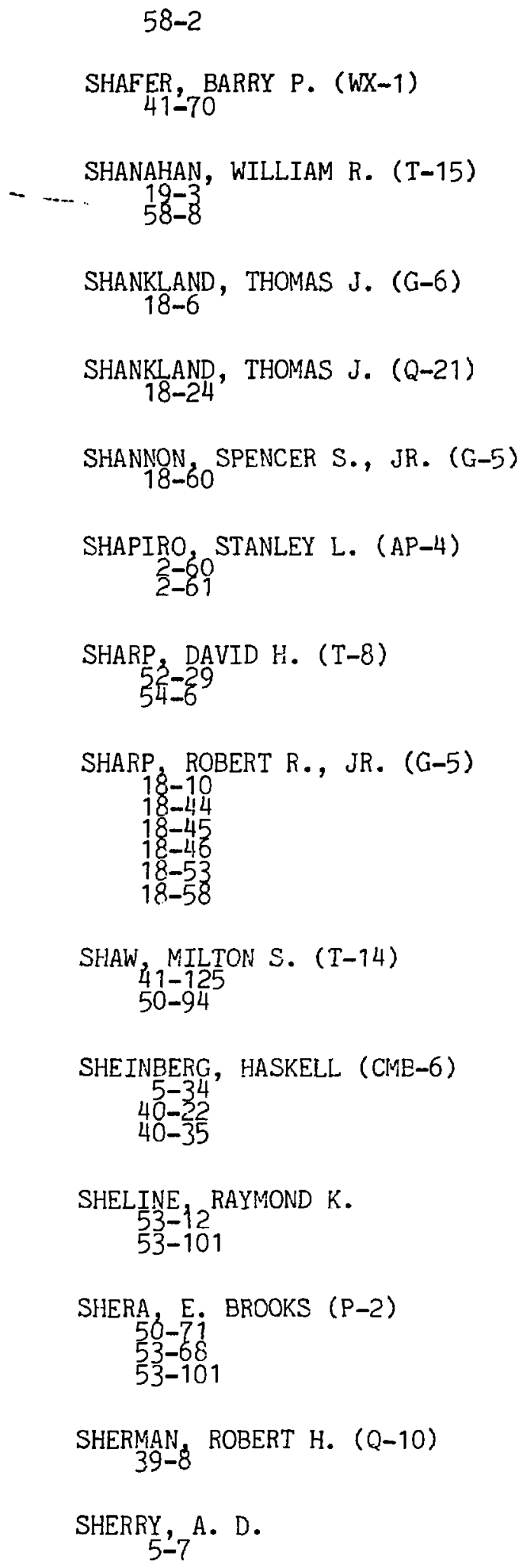




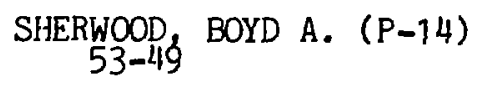

$\underset{26-4}{\text { SHIPLEY, JAMES } P .(Q-4)}$

SHIPLEY, JAMES P., JR. (Q-4)

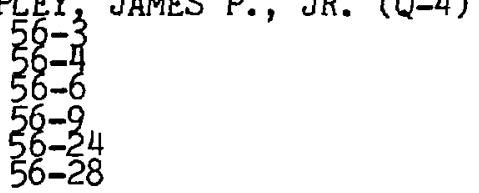

SHLAER, $\underset{41-57}{\text { SALLY }}$

SHREFFLER, L. COMP. $34-20$

SHIREFFLER, ROBERT G.
$28-7$

SHUNK, EDWARD R. (P-3)

$53-109$

SIBBITT, WILMER L., JR.

SIBERT, JOHN L. (C-6)
$46-2$
$46-10$

SIEMON, RICHARD E. (CTR-3)

19-11

$19-16$
$58-12$

$\underset{53-33}{\text { SIEMSSEN }}$ R. H.

SIERK, ARNOLD J. (T-9)

$53-62$
$53-63$

SIEVERS $\underset{32-70}{A . \mathrm{J}}$

SILBAR, RICHARD R. (T-5)

$\underset{53-24}{\text { SILBERT }}$ MYRON G. $(P-3)$

SILVER, RICHARD N. (T-11)
$52-27$
$52-28$

SIMMONS, JAMES E. (P-DOR)

$50-47$
$53-97$

53-104

${ }_{53-60}^{\text {SIMMONS }}$ JAMES E. $(P-7)$

SIMMONS, LEONARD M. (T-DO)

SIMONS, DAVID J.

SIMONS, SCOTT W.

$32-85$

$\underset{32-50}{\text { SIMONS, SCOTT W. (L-9) }}$

$\underset{32-36}{\text { SIMONSIC, GARY A. (CMB-6) }}$

SIMPSON $_{53-20}$ H. MITCHELL

SINGER,

SKAGGS, SAMUEL R. (CMB-3)
$\begin{gathered}40-24 \\ 57-7\end{gathered}$

$\underset{32-56}{\text { SKOBERNE, FREDERICK (L-DO) COMP. }}$

SKOGEN-HAGENSON, MARY JANE $(\mathrm{H}-10)$

SLAUGHTER, MILTON D. (T-DO)
$53-29$

SLAUGHTER, MILTON D. (T-8)

$52-30$
$54-12$

SMALE ${ }_{18-67}^{\text {RICHARD F. }}(\mathrm{H}-1)$

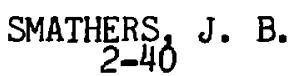


SMITH, DARRYL B. (Q-4)

$26-4$
$56-3$
$56-4$

$16-15$
$16-28$

SMITH $_{3}$ DAVID M. $(\mathrm{H}-4)$

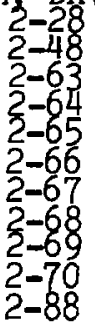

SMITH, ROBERT E. $(M-1)$

SMITH $_{38-7}$. T.

SMITH $_{2-34}$ TEMPLE F. (T-7)

$\mathrm{SMITH}_{56-32} \mathrm{~W}^{2} \mathrm{JOHN}$, II $(\mathrm{H}-1)$

SMITH, DAVID R. (Q-14)

SMITH, W5. JOHN, II (H-12)

$56-26$
$59-3$

SMITH, WILLIAM ROSS, JR.

SMITH, ELTON (MP-10)

SMITH, ${ }_{5}$ GORDON M. $\cdot(\mathrm{J}-10)$

SMYTH, JOSEPH R. (G-6)

SNOW, EDWARD CLARK (CME-5) $55-10$

SMITH ${ }_{53}-84$

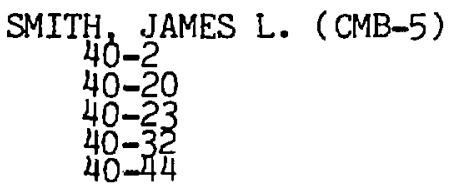

SNYDER, ELIZABETH A. (Q-12) COMP. $27-6$
$27-7$
$27-8$

SNYDERMAN, NEAL 54-13,

SMITH $_{53-84}$ E.

SMITH $_{38-7}^{\text {LARON L. (Q-7) }}$

SMITH $_{3-3}$ LOUIS C. (WX-2)

SOBOTKA, ANNE K.

SOFTWARE DOCUMENTATION GROUP

$$
\begin{aligned}
& 41-87 \\
& 41-88 \\
& 41-89
\end{aligned}
$$

SOLEM, ANN (C-6)

$$
\begin{aligned}
& 41-54 \\
& 41-91
\end{aligned}
$$

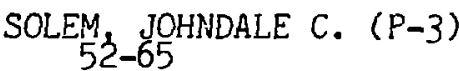

SOLLID, JON E. $(L-10)$

SMITH, PAUL D. $(Q-13)$
$11-7$
$33-10$

SMITH, PHILLIP R. 
SORAN, PATRICK D. (TD-6)

SOUKUP, J.

$53-3$
$53-5$

SPALDING, JOHN F. (H-4)

SPEIR,
$\substack{26-4 \\ 56-16}$

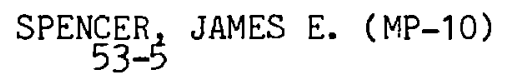

SPENCER, JAMES E. (MP-4)

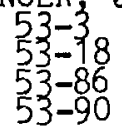

SPENCER $_{41-57}$ NANCY C.

SPIRIQ, CARMELO A. (Q-1)

$\underset{27-2}{\text { SPOONER }}$ JAMES E. (ENG-DO)

$\underset{2-60}{\text { SPOONESOWER, J. }}$

SPRINGER, ROBERT W. (CMB-6)

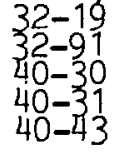

SPRINGER, THOMAS E. (E-4) $48-3$
$48-4$
$48-9$

SRINIVASAN,
33-11

ED.

STAFFORD, RONALD G. $(\mathrm{H}-1)$

STAMATELATOS, MICHAEL. G. $26-8$
$53-7$ $\underset{30-10}{\text { STAMPFER }}$ JOSEPH F., JR. $(\mathrm{H}-5)$

STARK, EUGENE E. (L-5)

$32-13$

32- 15

$32-68$

STARK, EUGENE E. (L-5) COMP.

STARK, WALTER A., JR. (CMB-3) $40-7$
$52-11$

STARKOVICH, VICTOR S. $(Q-8)$
$38-10$

$\underset{53-69}{\text { STARNER, JOHN W. }}(\mathrm{P}-2)$ STEBBINGS, JAMES H., JR. (H-DO)
$30-30$

STECHEL, ELLEN B.

$$
\begin{aligned}
& 50-9 \\
& 50-86 \\
& 50-105
\end{aligned}
$$

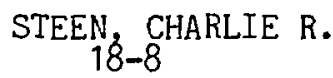

STEFFEN, ROLF M.
$\begin{gathered}50-71 \\ 53-68\end{gathered}$

STEIN, D. F.

STEIN, LELAND R. $(T-3)$
$16-20$
$41-74$

STEIN $_{2-86}$ MYRON L. (C-3)

STEIN, NELSON (P-9)

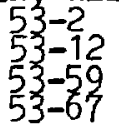

STEIN, PAUL R. (T-7)

$$
\begin{aligned}
& 41-75 \\
& 41-76 \\
& 41-77 \\
& 52-46
\end{aligned}
$$


STEINHAUS, DAVID W. (CMB-1)
$18-18$

STEINKAMP, JOHN A. (H-10)

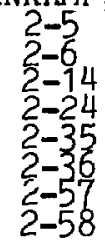

STEINKE, ROBERT G. (Q-7)

$38-7$

STELL $\underset{52-64}{\text { GEORGE }}$

STENBAEK-NIELSEN, H. C. $51-52$

STEPHANI, EVON L. (Q-22)

STEPHENSON, GERARD J., JR. (T-5) $53-1$
$53-37$
$53-106$

STERNHEIM, MORTON M. $53-53$

STEUERWALT, MICHAEL (C-3) 41-9

STEVENS ${ }_{47-7}$ RALPH R., JR. (MP-12)

STEVENSON, ANITA $(\mathrm{H}-10)$

$$
\begin{array}{rl}
2 & -23 \\
2 & 3 \\
2 & 38
\end{array}
$$

STEVENSON, MICHAEL G. (Q-DO) COMP. $26-14$
$26-15$
$38-5$

$\underset{50-35}{\text { STEWART }}$ BRUCE $(A P-4)$

STEWART, BRUCE S.

STEWART GREGORY R. ( CMB-3)

$40-10$

$40-39$
$40-40$

STEWART, JOHN M.

$$
\begin{aligned}
& 2-11 \\
& 2-12
\end{aligned}
$$

STEWART, LEONA (T-2)

$53-40$
$53-51$
$53-102$

STEYERT, WILLIAM A., JR, (Q-10)

$16-16$

$16-18$

$16-2$

$16-24$
$40-15$

STEYERT, WILLIAM A., JR. (Q-10) COMP. $16-17$
$47-15$

STILES, GARDINER S. 51-37

STODDARD, STEPHEN D. (CMB-6) $40-13$
$40-43$

$40-42$

$\underset{2-60}{\operatorname{STOECKENIUS,~} \mathrm{w}}$.

STOKER, ALAN K. (H-8)

$18-31$

$18-68$

STONE ${ }_{16-16}$ J.

STORM, ELLERY ( $\mathrm{H}-1)$

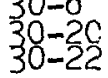

STORMS, EDMUND K. (CME-3)

$\underset{47-22}{\text { STOVALL }}$ JAMES E. (AT-1)

STRAIT, BOBBY G. $(L-10)$

$\underset{32-61}{\operatorname{STRATTON}}$ THOMAS F. $(L-10)$ 
STRAUB, GALEN K.
$50-78$

STRAUB, GALEN K. (T-4)

$$
\begin{aligned}
& 40-45 \\
& 50-28
\end{aligned}
$$

STREHL, VOLKER

$41-83$

STROH $\underset{4-85}{\text { KENNETH R. }}$ (Q-13)

STROSCIO, MICHAEL A. (L-6)

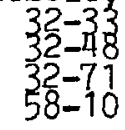

STRUEBING, VERNON O. (CMB-5) $40-20$
$40-32$

STUMP, CHARLES J., JR.

STUPIN, DAVID M. (L-7)

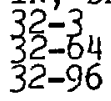

STUTZ, ROGER ALAN (ENG-8)

SUGAWARA, $K$. 40-26

SUHR $34-10$

SULLIVAN, ELIZABETH M. (H-DO) COMP.

SUNIER, JULES $W .(P-9)$

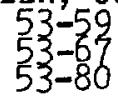

SUO-ANTTILA, A. J. (Q-7)

$\underset{51-28}{\text { SUTHERLAND, C. DEXTER }}(\mathrm{J}-10)$

SUTTON, JOHN G. (L-1)

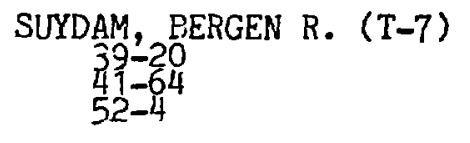

SUZUKAWA, HENRY H., JR.
$50-99$

SVITRA, ZITA V. $(\mathrm{H}-10)$

SWANSON,

SWANSON, DAVID A. (L-10)

$$
\begin{aligned}
& 32-5 \\
& 32-81
\end{aligned}
$$

SWANSON, ${ }_{5-35}^{\text {GERALD }} \mathrm{C}$.

$\underset{2-6 \uparrow}{\text { SWENBERG }}$ C. E.

SWENSON DONALD A. (AT-1)
$47-8$
$47-9$

SWENSON $_{2-54}$ DONALD A. $(M P-14)$

SWENSON $_{53-33}$ L. W.

SWENSON $_{53-70} W$

SZE, ROBERT C. (AP-2)

TAI, ${ }_{39-4}$ S. (CTR-12)

TALBERT 36 W. L., JR. (Q-14)

$$
\begin{aligned}
& 26-26 \\
& 36-3 \\
& 47-11 \\
& 56-18
\end{aligned}
$$

TALLEY, BEVERLY H. (MP-DO) COMP.
$27-12$

TANAKA, NOBUYUKI (MP-10) 
TAPE, JAMES W. (Q-1)

$5-24$

56-20

TAYLOR, HOWARD S.
50:-123

TAYLOR, JOHN W. (M-DO)

TAYLOR, PAUL M.

TAYLOR, THOMAS N. (CMB-8) $40-3$ $40-21$ $40-34$

TEASDALE, W. A. $(W X-4)$

TERCOVICH, RENATO G. (L-9)

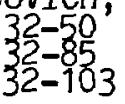

TERRELL ${ }_{51-18}$ JAMES $(P-4)$

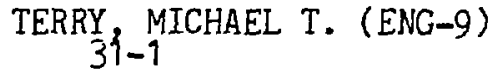

TESTER, JEFFERSON W. (G-3)

THERMAL REACTOR SAFETY GROUP 35-8

THIESSEN 2 HENRY A. (MP-10)

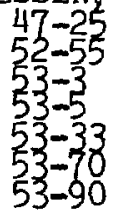

THODE, LESTER E. (T-15)

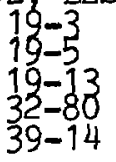

THOMAS, JOE T.
THOMAS, KEITH S. (CTR-7) COMP. $39-9$
$39-27$

THOMAS, RICHARD F., JR.

THIOMAS, ROBERT G. (H-H)

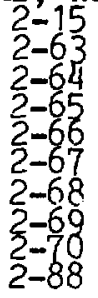

THOMAS, SCOTT J. (L-9)

$32-16$
$32-70$
$32-93$

THOMASON, MICHAEL D. (L-1)
32-98

THOMASSEN, KEITH I. (CTR-DO) $39-21$

THOMPSON, DONALD L. (CNC-2)

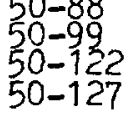

$\underset{40-16}{\text { THOMPSON, JOE D. }(Q-10)}$

THOMPSON, THOMAS K. $(\mathrm{H}-7)$

THOMSON, DAVID B. (P-3)

THOMSON, DEBRA ANN $(\mathrm{H}-1)$

$$
\text { 30-17 }
$$

THUOT, MICHAEL E. (E-4)

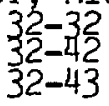

TIETJEN, GARY L. (Q-12)

$$
\begin{aligned}
& 2-51 \\
& 41-65 \\
& 56-27
\end{aligned}
$$

TIETJEN, GARY L. (S-1) 
5-36

TILLERY, MARVIN I. (H-5) $18-27$
$30-3$
$30-4$

TING $_{52-3} C_{0} . S$.

TINSLEY, J. $53-3$
$53-5$

$\mathrm{TJON}_{53-75}$ JOHN $(\mathrm{T}-5)$

TOBEY, ROBERT A. (H-9)

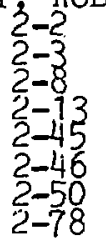

TODD $33-4$ BILLY E. $(G-4)$

TODD $_{18-4}^{\text {BILLY E. }}(Q-22)$

TORRES, AGAPITO P. $(w X-3)$

TORREY, MARTIN D. (T-3)

$$
\begin{aligned}
& 35-3 \\
& 49-86
\end{aligned}
$$

TRAPP, JOHN A.

TRAVIS, $\underset{2-25}{G} \cdot(H-10)$

TRAVIS, JOHN R. (T-3)
$38-7$
$52-20$

TREXLER, PAMELA K. $(G-5)$
$18-48$
$24-15$

TROTT, WAYNE M. (CNC-2)

TRUHLAR, DONALD G. (CNC-2)
$50-1$

TRUJILLO
$30-26$
$30-28$

TRUMP, MICHAEL A. (E-4)

$23-5$
$31-3$

TRUNDLE $36-32$. S.

TRUSSELL, HENRY J. (M-8)

$41-13$

$41-15$

$41-64$

$41-73$

$41-105$

$41-119$

TSCHALAR, C.

TUCKER, EDWIN K. (WX-8)

TUCKER, EVA F. (WX-4)

TUCKER, HOWARD Eे.
$32-57$

TURNER, A. KEITH (Q-10)

TURNER, LEAF (CTR-6)

TURNER, ROBERT D. (Q-10) $15-10$
$15-11$

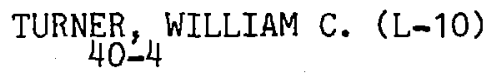

UMBARGER, C. JOHN (H-1)

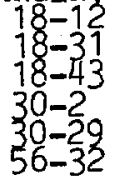

URIZAR, MANUEL J. (WX-2) 
VAHALA, GEORGE

39-22

VALENCIA, FLAVIO A. (CMB-3)

VALENTINE, ALLEN M. (H- 1)

VALLEY, MARCY

VAN DER BEKEN, HENRI

41-57

VAN DER LAAN, PIETER C. (CTR-DO)

$20-26$
$20-29$

VAN DYCK, OLIN B. (MP-13) COMP.

$47-17$

47-19

VANDERBORGH, NICHOLAS E. (CMB-8)

$$
\begin{array}{r}
7-2 \\
8-3 \\
46-1 \\
48-3
\end{array}
$$

VANDERBORGH, NICHOLAS E. (G-7)

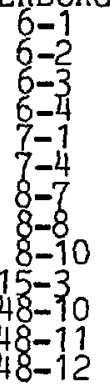

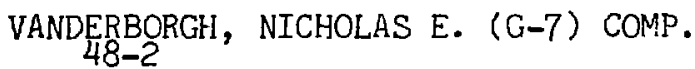

$\underset{5-2}{\text { VANDERZEE, CECIL }}$

VARGHESE, PHILIP

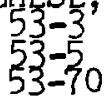

VASILIK, DENNIS G. (H-1)

$30-37$
$30-29$
VEESER, LYNN R. (P-3)

52-65

$53-15$
$53-72$
$53-89$

VERGNES, M. N.
$53-98$
$53-103$

VIDALE, ROSEMARY J. (CNC-11)
$18-34$

VIGIL, ARMANDO S. (WX-3)
$\begin{gathered}3 \rightarrow-6 \\ 4 \uparrow-118\end{gathered}$

VISSCHER, WILLIAM M.
$52-68$

VISSER, JAN W. M.

VISWANATHAN, V. K. (L-1)

32-72

VOEL $\frac{2}{30-30}$ GEORGE L. (H-DO)

VOGEL, HERBERT F. (CTR-9)
$20-2$
$20-6$
$20-20$
$41-09$

VOLKIN, HOWARD C. (L-5) $32-37$
$32-68$

VOORHEES, EDWARD A. (C-DO)

$\underset{52-14}{\text { VORTHMAN, }}$ J. E.

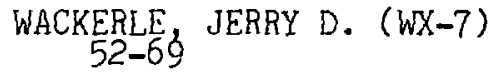

WADEHRA
$50-76$

WADT, WILLARD R. (T-12)

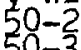

$50-36$

$50-3$

$50-689$ 
WAGEMAN WILLIAM E. ( CNC-4)

$5-19$
$5-25$

$50-79$

WAGNER, PAUL (CMB-8)

$$
\begin{aligned}
7-2 \\
8=7 \\
8-10 \\
25-2
\end{aligned}
$$

WAHMAN, LYLE A. (CMB-8)

WALKER, ROBERT B. (T-12)

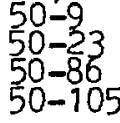

$50-100$

$50-101$
$50-102$

WANEK, PATSY L. (CMB-8)

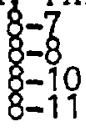

WANEK $3-35$ PATSY L. $(\mathrm{H}-10)$

WANEK $_{3-77}$ PHILIP M. (CNC-11)

WANG $_{40-23}$ RUN-HAN (Q-10)

WALKER, THOMAS E. (CNC-4) $50-26$

$50-117$

WALLACE

WALLACE ${ }_{53-91}$ JON M. (TD-2)

WALLER, RAY A. (S-1) $38-3$

WANG, SUSIE Y. $(\mathrm{H}-4)$

WANGEN, LAWRENCE E. (CMB-8)

WANGEN, LAWRENCE E. (H-8)

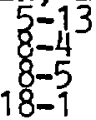

WARNER, CHARLES L. $(\mathrm{H}-7)$

WARREN JOHN L. (Q-DO) ED. 26-19

WARREN, JOHN L. (Q-DO)

WARREN, JOHN L. (Q-DO) ED. $26-18$
$26-21$

WALTERS, WALLACE F. (T-1)
$36-4$

WALTON, RODDY B. (Q-1)

$$
\begin{aligned}
& 45-14 \\
& 56-10
\end{aligned}
$$

WARREN, JOHN LAWRENCE (H-7)
$45-8$

WARREN, NICHOLAS W. (Q-21)

WARREN, RICHARD G. (G-5) $18-46$
$18-49$

WAMPLER , FRED B. (AP-3)

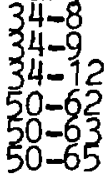

WARREN, ROGER W. (CTR-9) 
WATERBURY, GLENN R. (CMB-1)

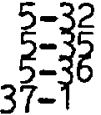

WATERBURY, GLENN R. (CMB-1) COMP. $30-13$
$30-12$
$30=13$
$30=14$
$56-1$

WATERMAN, MICHAEL S. (Q-12)

$$
\begin{aligned}
& 2-34 \\
& 41-6 \\
& 41-26 \\
& 41-39 \\
& 56-25
\end{aligned}
$$

WATERMAN, MICHAEL S. (S-1) $2-82$
$48-7$

WATKINS $\underset{32-41}{\text { DAVID } W .(T D-2)}$

WEATHINGTON, CARL $41-18$

WEAVER, THOMAS A. (G-5) $18-17$
$18-48$
$18-62$

WEBER JOE

WEBER JOSEF (CNC-11)

WEBER, WILLES $\mathrm{H}$.

WECKSUNG GEORGE W. (M-8)

$$
\begin{aligned}
& 41-191 \\
& 44-1 \\
& 44-2 \\
& 44-3
\end{aligned}
$$

WECKSUNG, MONA J. (Q-12) $8-2$
$4 \uparrow-50$

WEEKS, ROBERT W. $(\mathrm{H}-5)$

WEERTMAN $_{40-28}$ JOHANNES (CME-8)
WEINER, RICHARD (T-DO)
$\begin{gathered}54-2 \\ 54-7\end{gathered}$

WEINSTOCK, $\mathrm{H}$. $52-49$
$52-50$

WEISEIN ${ }_{53-99}$ C. R.

WEISS, PAUL B. (L-9)
$32-41$
$32-98$

WELLS, ALAN HARVEY

$\underset{32-66}{\text { WELLS, FRANK D. (E-5) }}$

WELLS, MARK B. (T-7)

$\underset{51-52}{\text { WESCOTT }}$ EUGENE M.

WEST $\underset{24-10}{\text { FRANCIS G. }}(\mathrm{G}-6)$

WEST FRANCIO G. (G-9) $18-32$
$18-42$

WEST LEON, JR. $(H-1)$
$18-12$
$30-2$
$56-32$

WESTRIMM, EDGAR F., JR.

WEWERKA, EUGENE M. (CMB-8)

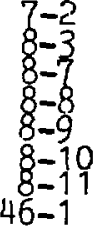

WHALEY, $\frac{1}{5}-4 \frac{1}{1}$ HOMAS $\mathrm{w} \cdot(\mathrm{H}-11)$

WHEAT, BILLIE M. (WR-8) 


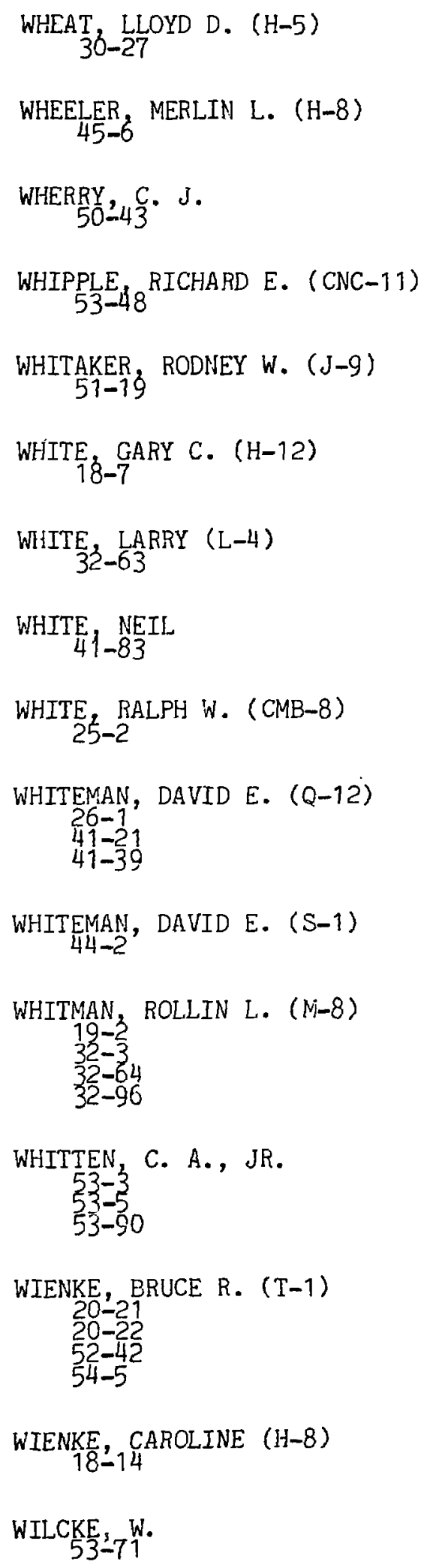

WILD ${ }_{53-26}^{\mathrm{J}} \mathrm{F}$. WILEY RICHARD A. (C-3)
$\begin{gathered}41-50 \\ 41-127\end{gathered}$

WILHELMY, JERRY B. (CNC-11) 53-26

WILKINS, RONALD W. (CTR-8)
$\begin{gathered}19-14 \\ 41-82\end{gathered}$

NILLBANKS, EMILY W. (C-4)

WILLCUTT, GORDON J. , JR. (Q-6)
35-1,
$35-4$

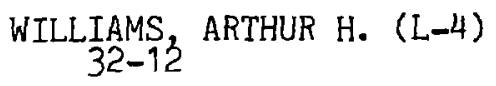

WILLIAMS, JOEL M. (CME-8)

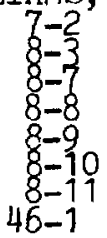

WILLIAMS, KEN A. (Q-6)

$35-7$

WILLIAMS, MICHAEL D.

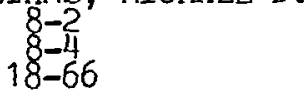

WILLIAMS, RAY E. (G-4)

WILLIAMS, ROBERT A. (CNC-11)
$50-91$
$53-34$

WILLIAMSON, KENNETH D., JR. (CTR-9) $15-4$
$20-15$

WILSON, JULIE S. (H-4)

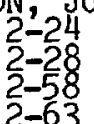




$$
2-64
$$

WILSON, JULIE S. $(H-4)$

WILSON, SAMUEL K. $(\mathrm{H}-8)$ $18-22$
$18-37$

WILSON, WILLIAM B. (T-2) $53-64$
$53-77$

WING, ${ }_{47-66}$ MILTON $(T-7)$

WINN, $\underset{2-61}{\text { KENNETH R. }}(\mathrm{AP}-4)$

WINSKE, DAN (CTR-6)
$58-9$

WIPF, STEFAN L. $(Q-10)$

$\underset{18-25}{\text { WIREMAN }}$ D. L.

WITTE ${ }_{2\}-18}$ KATHLEEN H. (C-3)

WOF SY $_{2-84}$ CARLA

$\underset{2-17}{\text { WOHLERS }}$ M. R.

WOHLETZ $18-42$ KENNETH H. $(G-6)$

WOHLFAHRT, HANS D. (P-2)
$53-68$

$\underset{51-6}{\text { WOLCOTT, JOHN H. }(J-10)}$

WOLF, MICHAEL ALLEN $(E-1)$

WOLFE $33-37$ PETER N. $(L-10)$

WOLF SBERG, KURT (CNC-11)
$18-38$
WOLFSBERG, MAX (CNC-2)
$50-99$

$W O O D_{57-i} B$.

WOOD $\underset{30-9}{\text { GERRY }}$ O. (H-5) PROJECT DIRECTOR

WOOD $\underset{50-54}{\text { JOHN }}$ H. (CMB-5)

$\underset{20-17}{\text { WOODRUFF, GENE L. }}$

WOODS, CLIFFORD W. $(p-9)$

$53-59$
$53-67$ WOODS, RICHARD (P-9) COMP.
$47-26$

WORLTON, WILLIAM J. (C-DO)

$41-25$

41-122

WORMAN, FREDERICK C. V.

WORTH, GARY M. (Q-2)

WRIGHT, BRADFORD L. (CTR-1)

WUNDER, RAINER

YAMAZAKI, Y. $(P-2)$

$50-71$
$53-68$
$53-101$

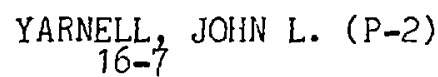

YARNELL, JOHN L. $(P-8)$

YASUDA, STANLEY K. $(H-5)$
$30-27$

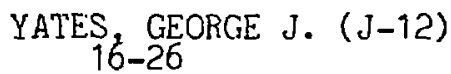


$\underset{32-5}{\text { YAVORNIK, EDWARD J. }(L-10)}$

YORK $_{30-30}$ DONALD A. (H-DO) $\because$

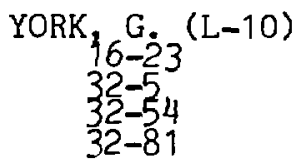

$\underset{18-36}{\text { YOUNG }}$ L.

YOUNG, PHILLIP G., JR. (T-2)

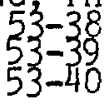

YOUNG, PHILLIP G., JR. (T-2) COMP.

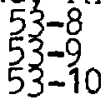

$\underset{53-30}{\text { YOUNG, STEPHEN K. }}(\mathrm{T}-5)$

ZAIDER, MARCO (NP-3)

$2-49$

$52-58$

ZEIDMAN, B.

$53-33$

ZEIGLER ${ }_{5-35}$ ROYAL K. (CMB-1)

ZEIGLER, ROYAL K. (Q-12)

44-3

ZEIGLER ${ }_{5-36}$ ROYAL K. (S-1)

ZEIGNER, VERNON L. (L-10)

$32-25$
$32-61$

ZEMACH, CHARLES (T-DOT)

$52-29$

ZERWEKH, AL (CMB-1)

$45-4$
$45-5$
$45-14$
ZIFF, ROBERT M. $(Q-10)$

$52-7$
$52-66$

ZIMMERMANN, EUGENE L. (L-1) $32-90$

$\mathrm{ZINN}_{51-28}$ JOHN $(J-10)$

$\mathrm{ZINN}_{57-i} \mathrm{~K}^{-}$

ZOCHER, ROY W. (CMB-11)

55- -30

ZOLLER, WILLIAM H.

ZVITRA, ZITA V. $(\mathrm{H}-10)$ 


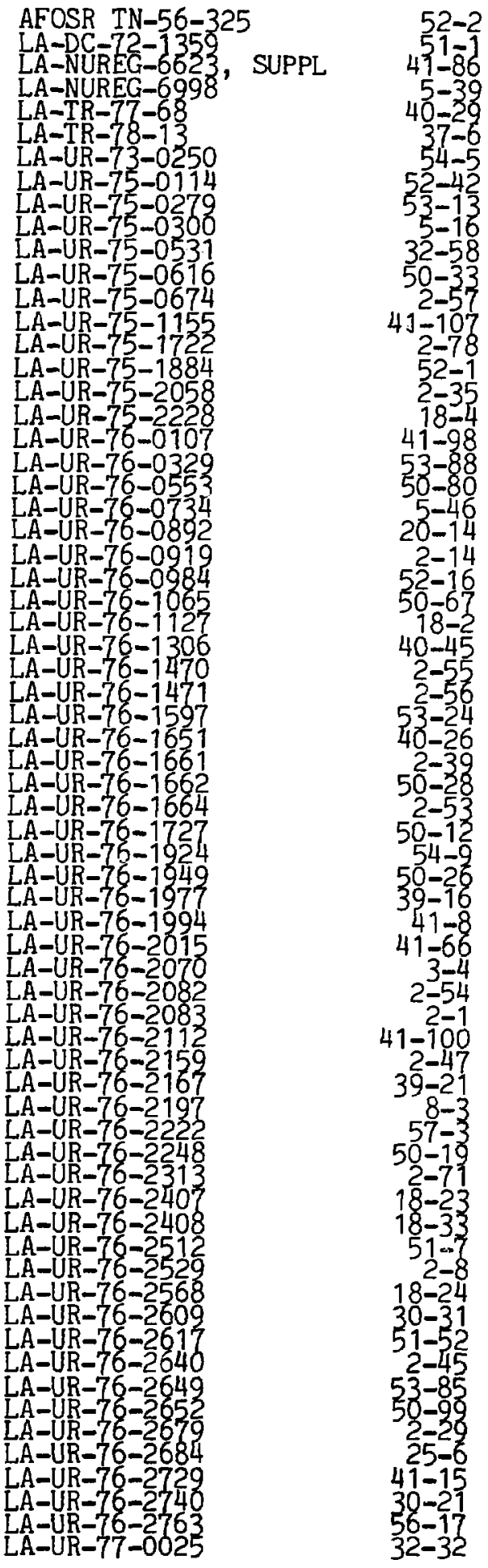

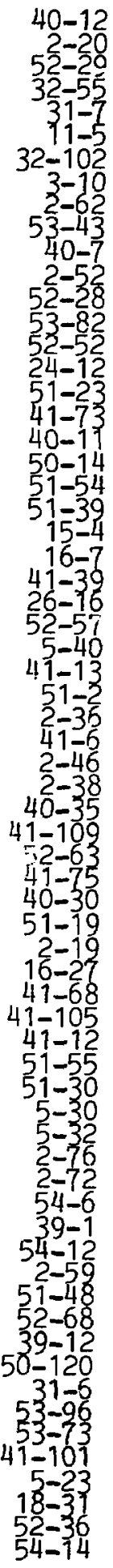




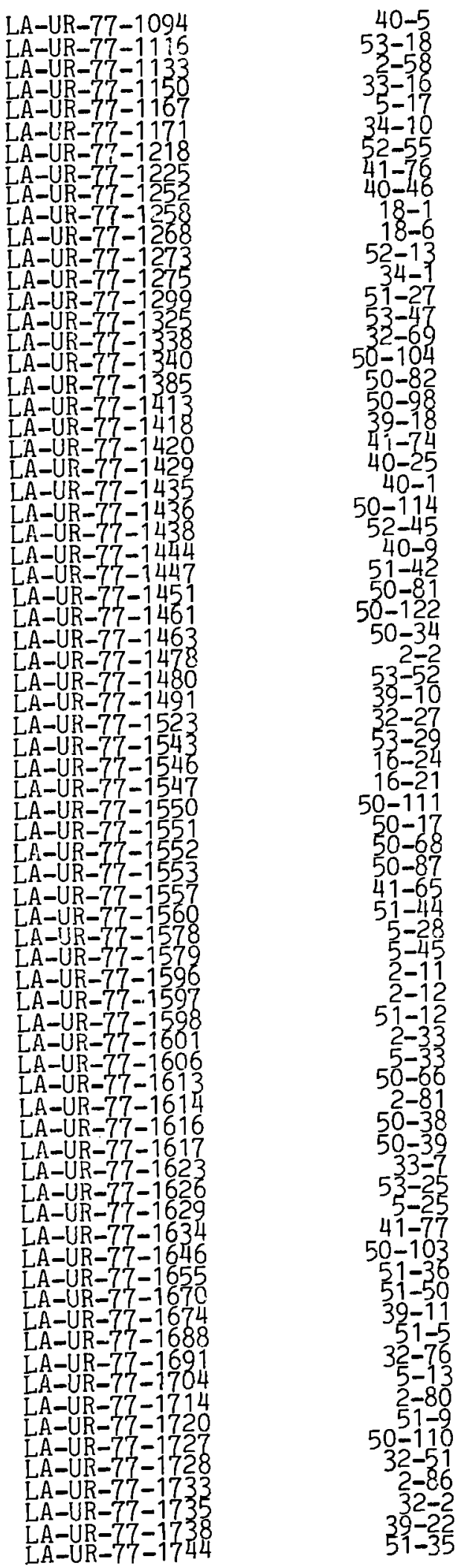

LA-UR-77-1752
LA-UR-77-1754
LA-UR-77-1764
LA-UR-77-1779
LA-UR-77-1785
LA-UR-77-1786
LA-UR-77-1789
LA-UR-77-1841
LA-UR-77-1845
LA-UR-77-1847
LA-UR-77-1854
LA-UR-77-1856
LA-UR-77-1866
LA-UR-77-1868
LA-UR-77-1875
LA-UR-77-1876
LA-UR-77-1880

LA-UR-77-1887

LA-UR-77-1888

LA-UR-77-1803

LA-UR-77-1894

LA-UR-77-1902

LA-UR-77-1906

LA-UR-77-1914

LA-UR-77-1920

LA-UR-77-1930

LA-UR-77-1937

LA-UR-77-1962

LA-UR-77-1964

LA-UR-77-1974

LA-UR-77-1976

LA-UR-77-2003

LA-UR-77-2008

LA-UR-77-2009

LA-UR-77-2014

LA-UR-77-2017

LA-UR-77-2023
LA-UR-7 -2027

LA-UR-77-2029

LA-UR-77-2030

LA-UR-77-2034
LA-UR-77-2038

LA-UR-77-2040

LA-UR-77-2045
LA-UR-77-2048

LA-UR-77-2049

LA-UR-77-2051

LA-UR $-77-2052$
LA-UR-77-2054

LA-UR-77-2058

LA-UR-77-2063

LA-UR-77-2064

LA-UR-77-2066

LA-UR-77-2069

LA-UR-77-2072

LA-UR-77-2081

LA-UR-77-2082

LA-UR-77-2083

LA-UR-77-2097
LA-UR-77-2100

LA-UR-77-2106

LA-UR-77-2110

LA-UR-77-2120

LA-UR-77-2126

LA-UR-77-2127

LA-UR-77-2128

LA-UR-77-2129

LA-UR-77-2 130
LA-UR-77-2131 

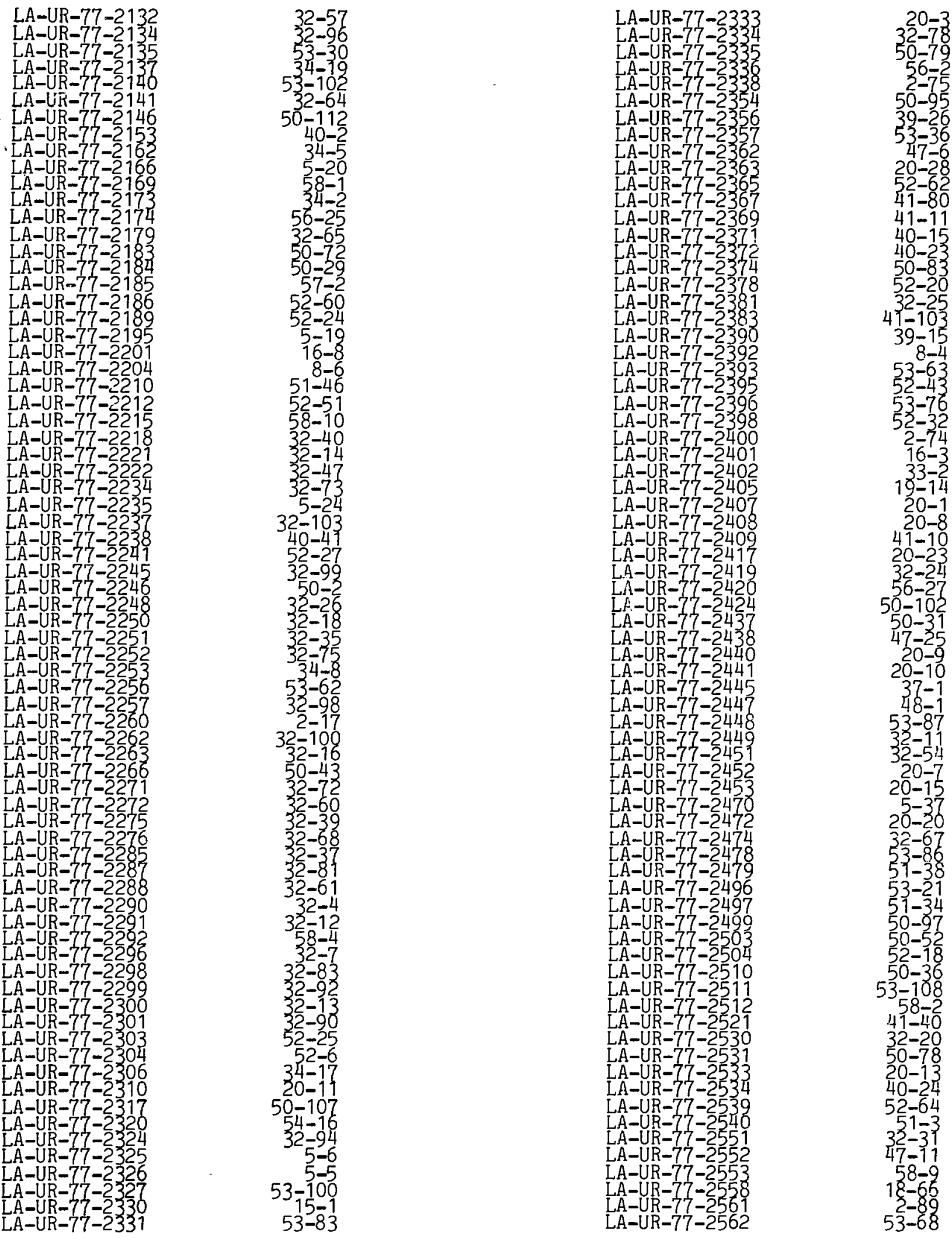


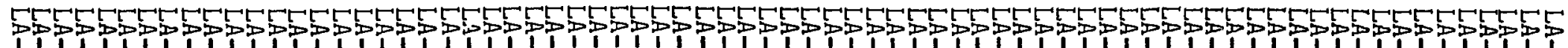

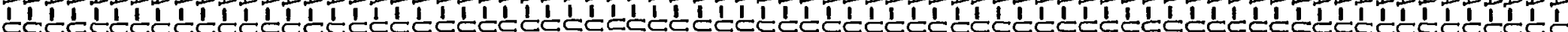

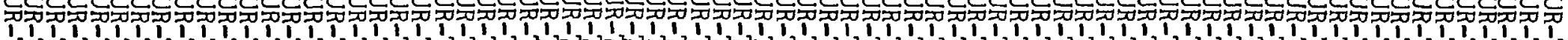

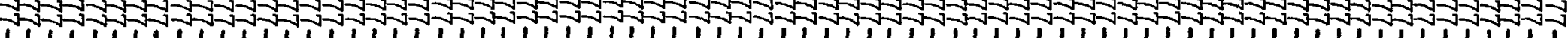

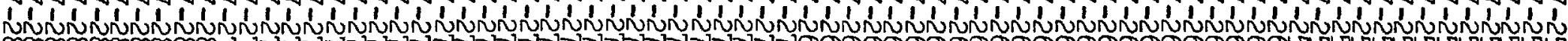

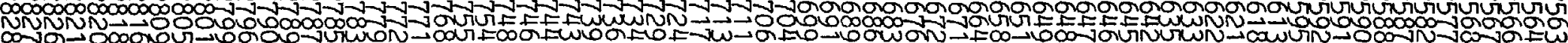

un

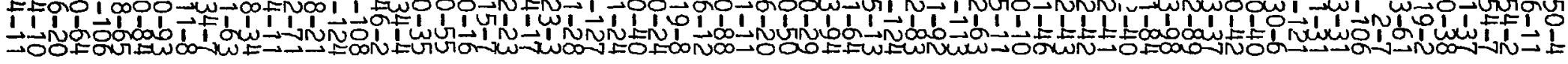

ঙ요

年5

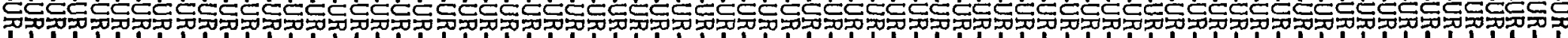

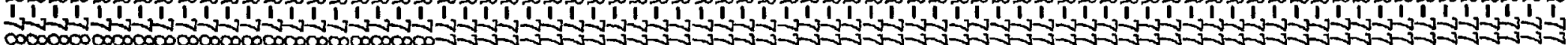

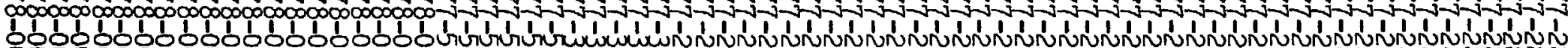

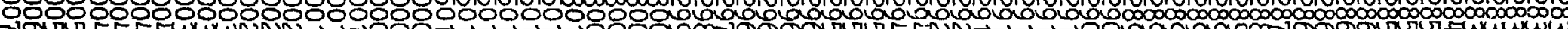

$\exists g u$ J

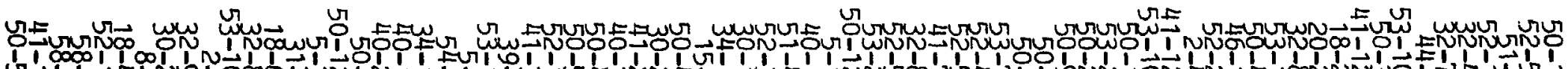

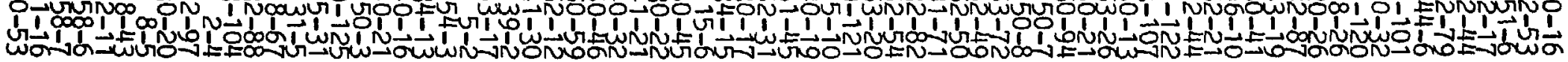




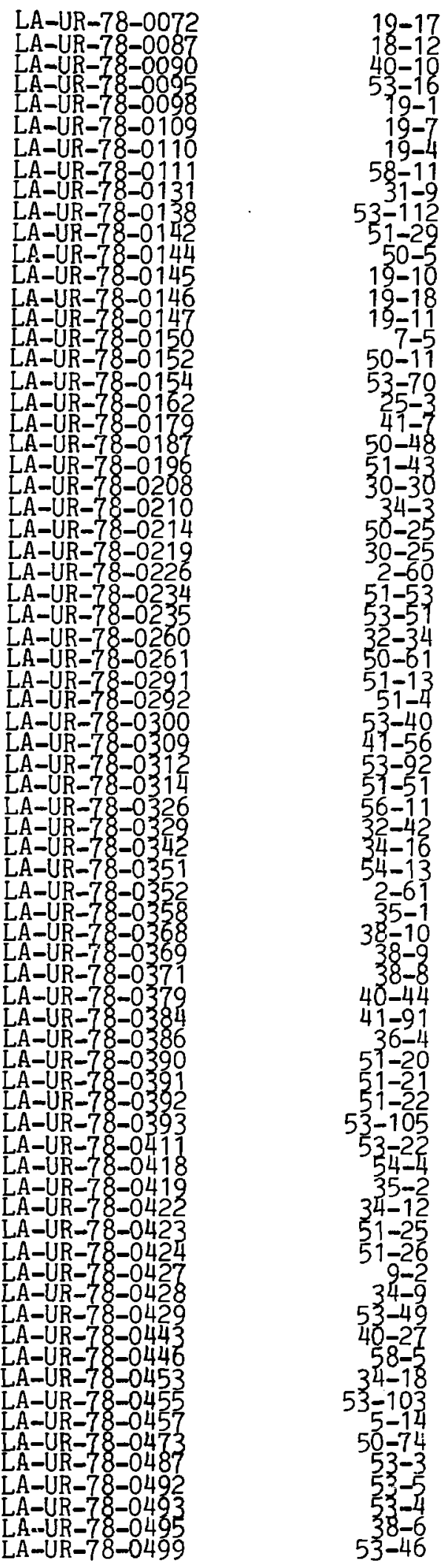

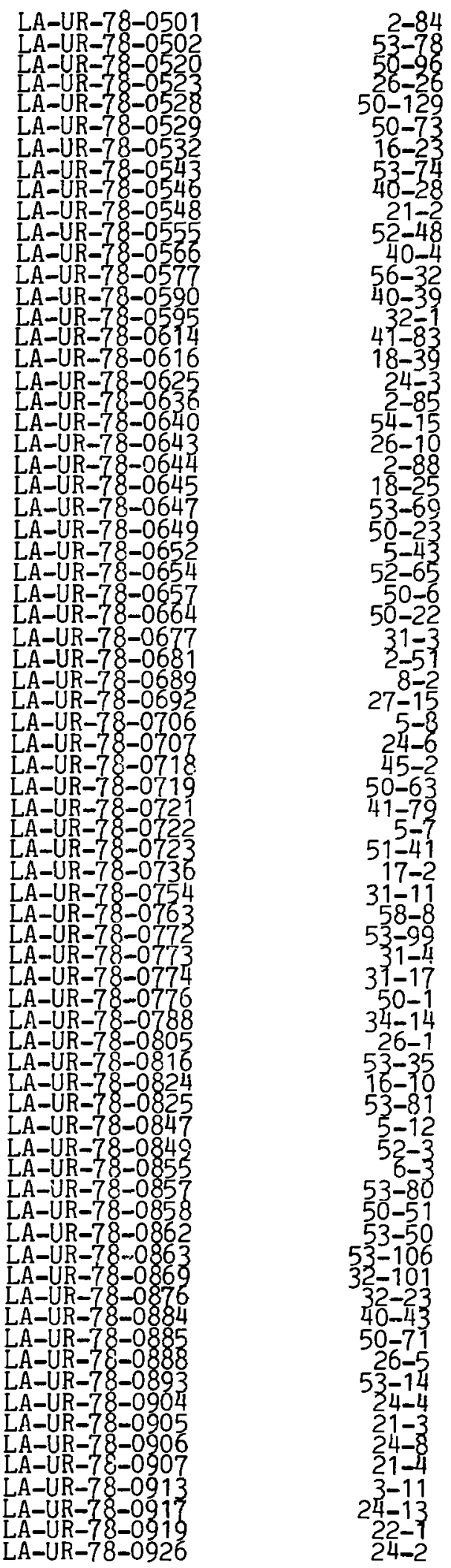




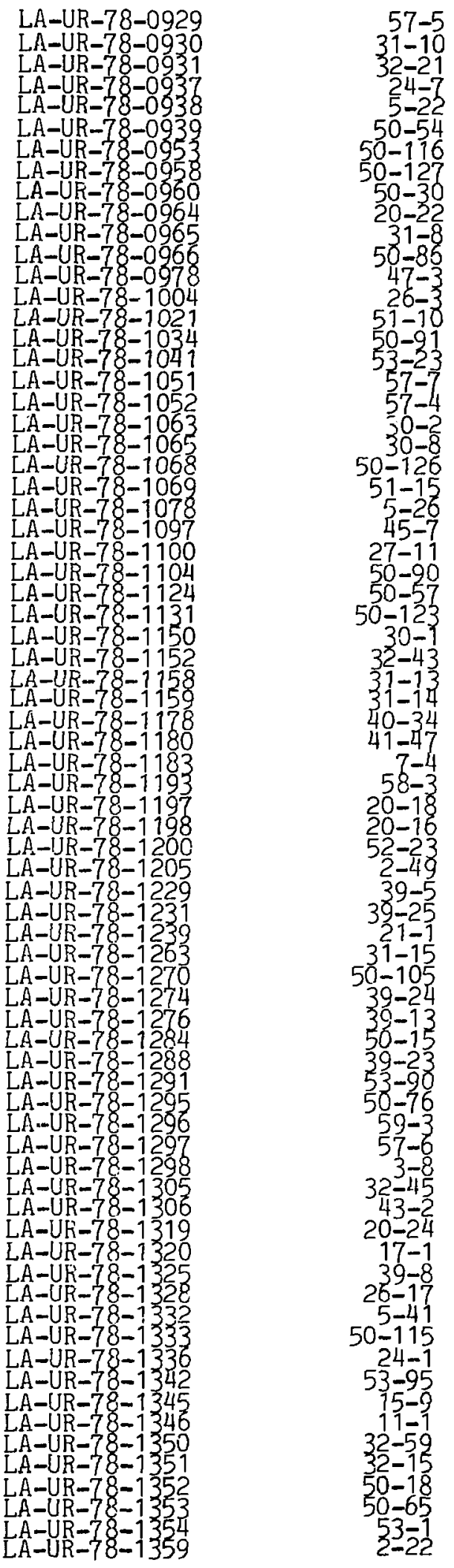

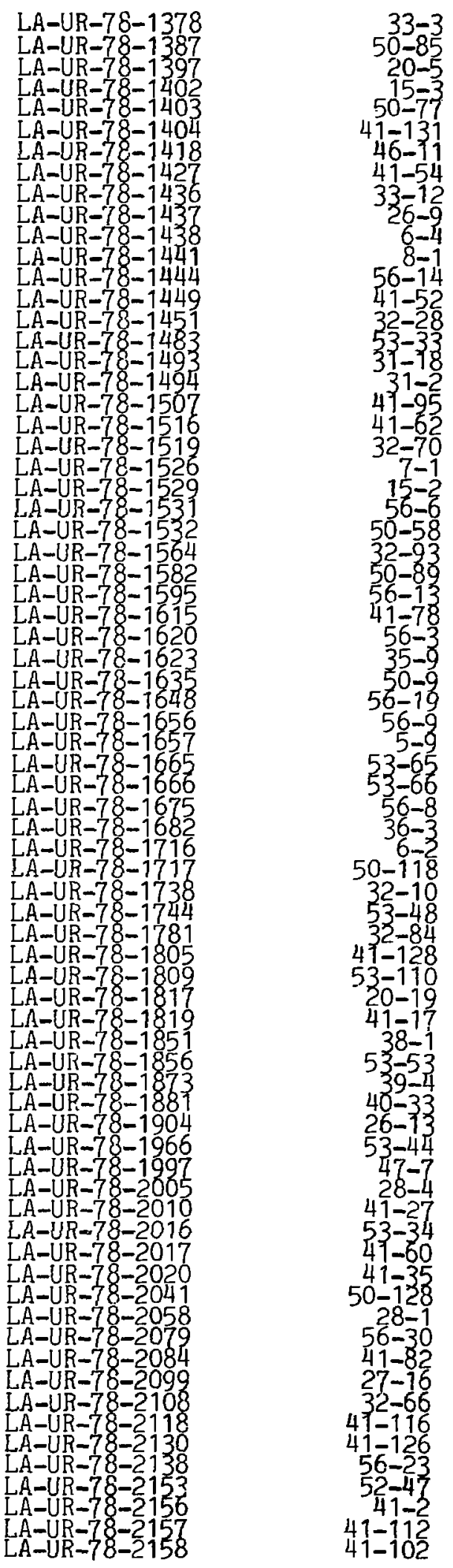



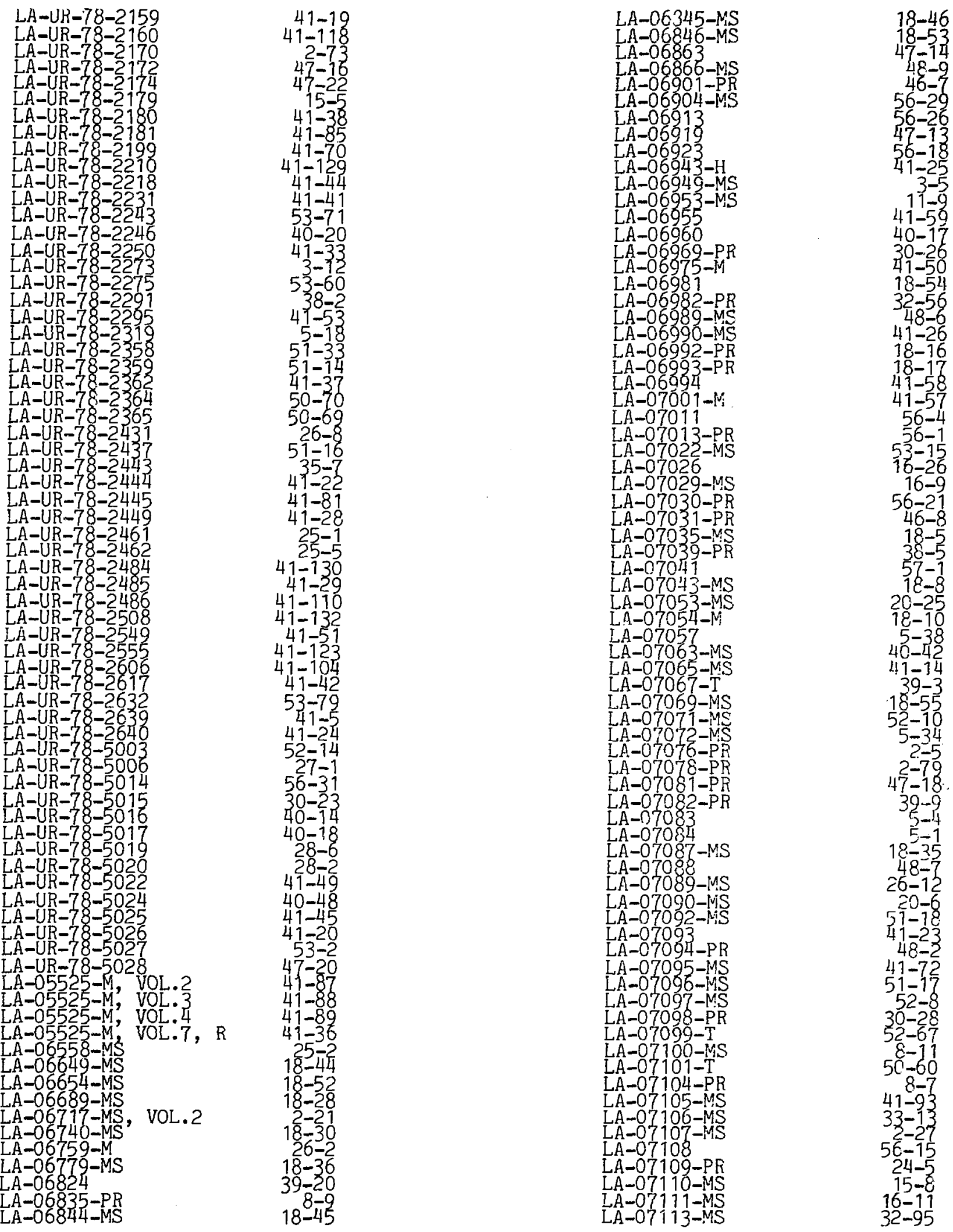

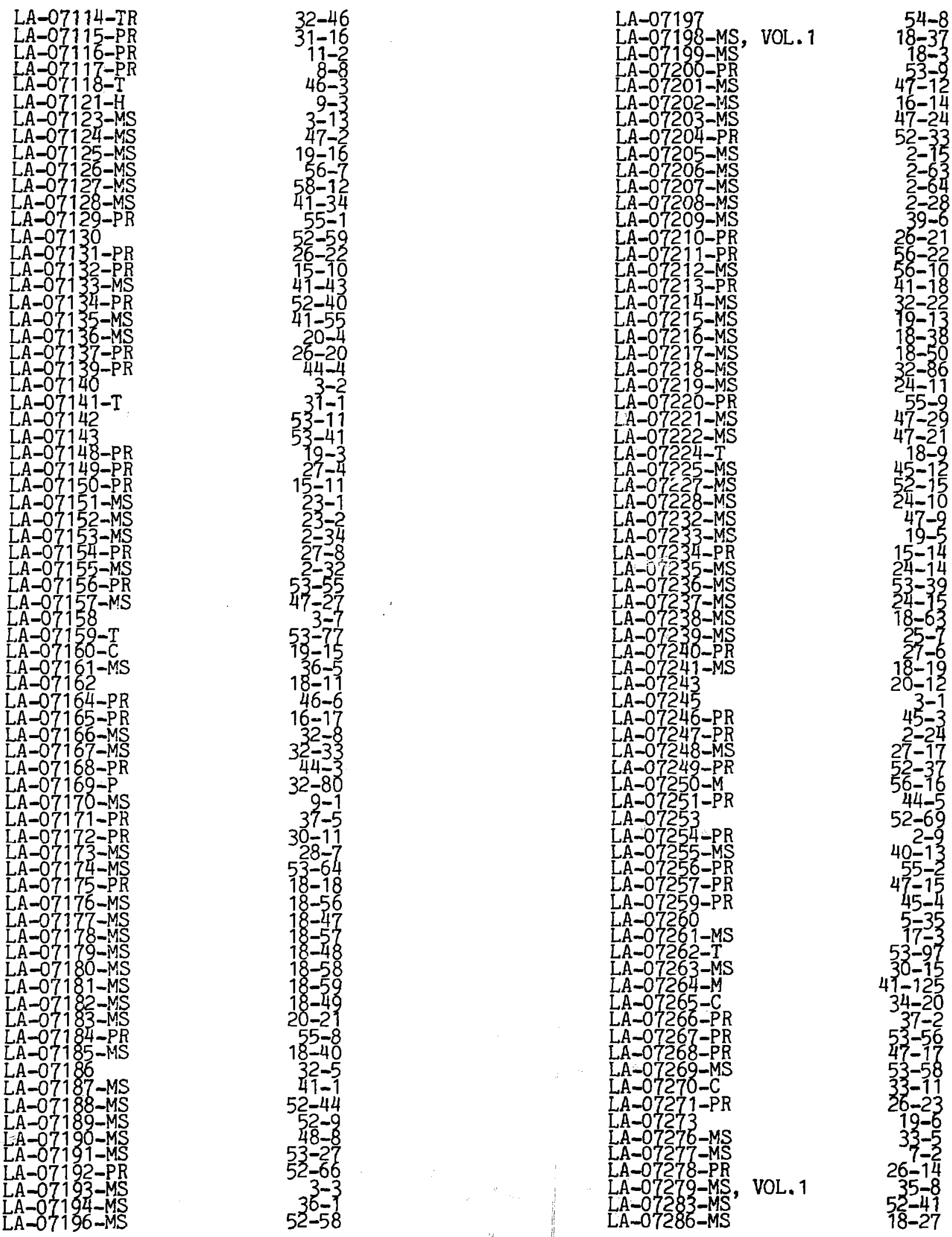


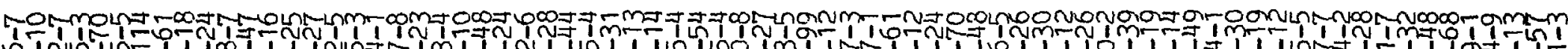

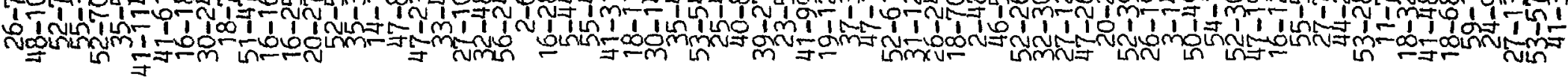

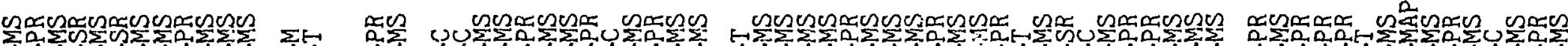

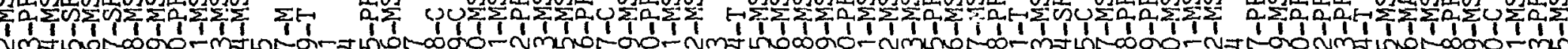

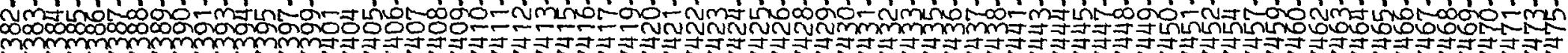

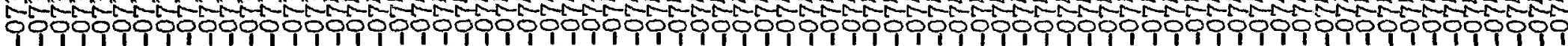

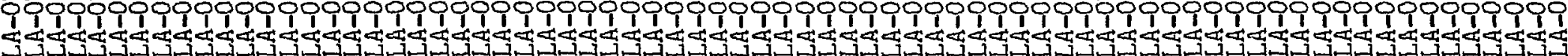

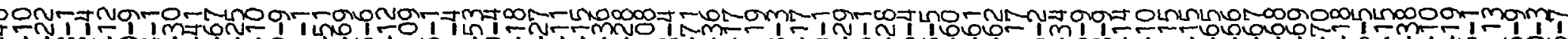

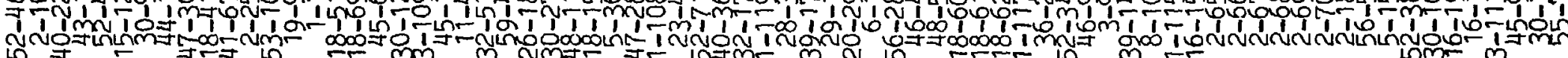

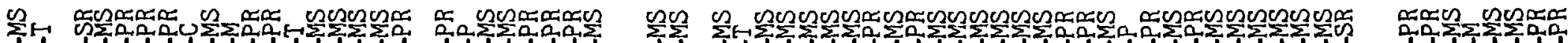

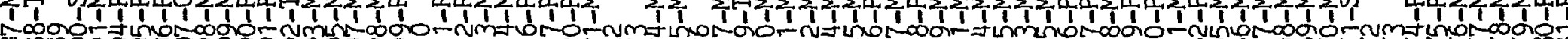

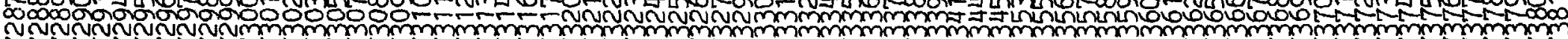

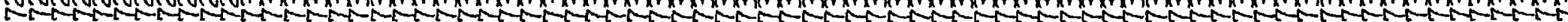

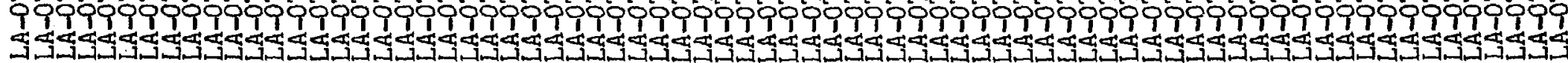



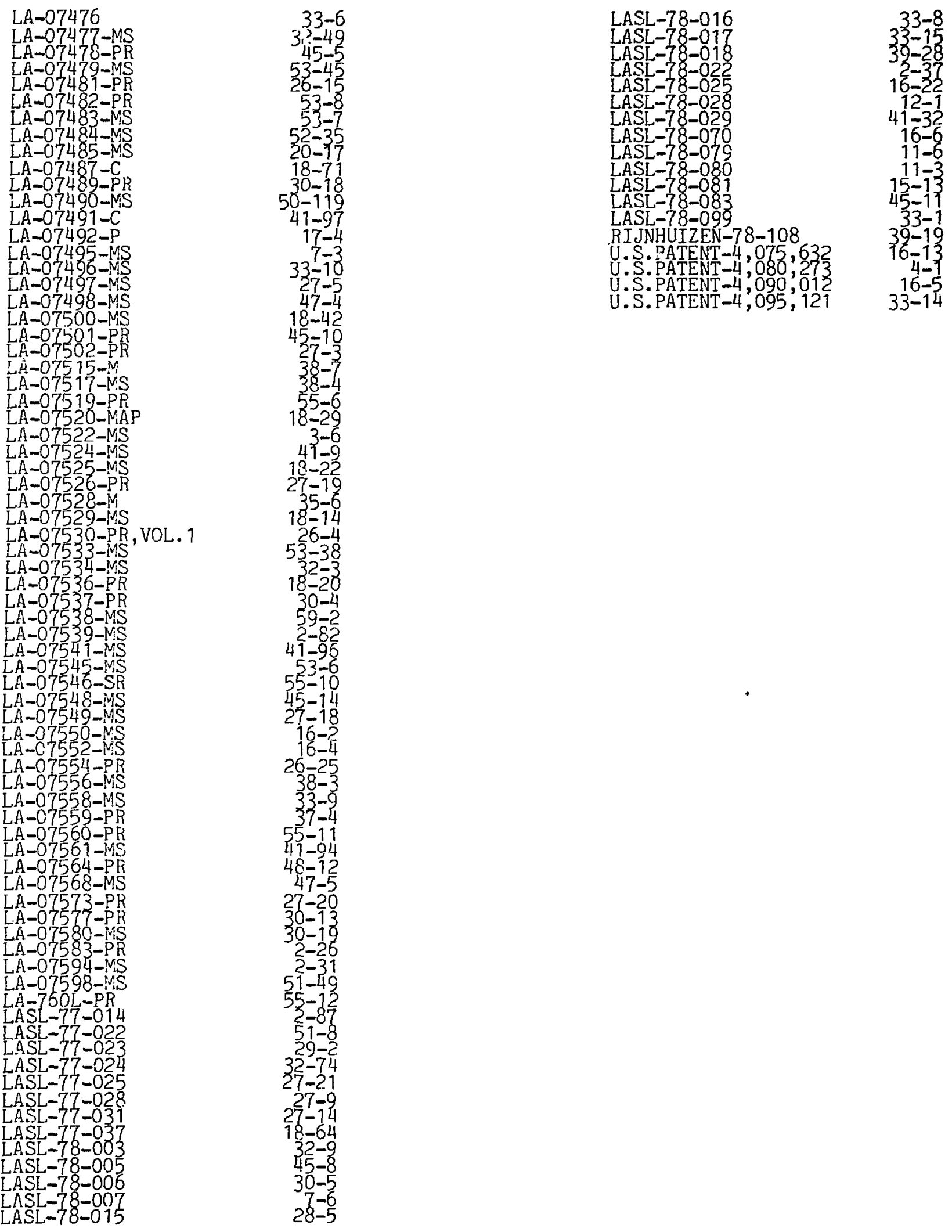
PUBLICATION TABULATION

A. JOURNAL ARTICLES-489 articles in 160 journals.

ACTA CRYSTALLOGRAPHICA--2

ADVANCES IN CRYOGENIC

ENGINEER ING - 3

AMERICAN INDUSTRIAL HYGIENE

ASSOCIATION JOURNAL -5

AMERICAN JOURNAL OF PHYSICS-]

ANALYTICA CHIMICA ACTA-1

ANALYTICAL CHEMISTRY-3

ANALYTICAL LETTERS-1

ANNALS OF PHYSICS-1

APPLIED OPTICS-11

APPLIED PHYSICS LETTERS-9

APPLIED SPECTROSCOPY - 1

ARMA RECORDS MANAGEMENT

QUAR TERLY - 1

ASTRONOMY AND ASTROPHYSICS-1

ASTROPHYSICAL JOURNAL-10

ASTROPHYSICS AND SPACE SCIENCE-I

BIOCHEM ISTRY - 4

BIOCHIMICA ET BIOFHYSICA ACTA-1

BIOPHYSICAL CHEMISTRY-1

BIOPHYSICAL JOURNAL-3

BRITISH JOURNAL OF CANCER-1
BRITISH JOURNAL OF RADIOLOGY-4

EULLETIN OF THE AMERICAN

ASTRONOMICAL SOCIETY-I

BULLETIN OF THE AMERICAN CERAMIC SOCIETY -1

CARBOHYDRATE RESEARCH-1

CERAMIC INOUSTRY -1

CHEMICAL PHYSICS LETTERS-7

COMMENTS ON NUCLEAR AND PARTICLE PHYSICS-1

COMMUNICATIONS OF THE ACM-1

COMPUTER METHOOS IN APPLIED MECHANICS AND ENGINEERING-I

COMPUTER PHYSICS COMMUNICATIONS- 1

COMPUTERS IN BIOMEDICAL RESEARCH-I

COORDINATION CHEMISTRY REVIEWS- 1

DISCRETE MATHEMATICS-1

ENERGY - 1

ENVIRONMENTAL SCIENCE AND TECHNOLOGY-2

EUROPEAN JOURNAL OF BIOCHEMISTRY -1

EXPERIMENTAL CELL RESEARCH-3

GEOLOGICAL SOCIETY OF AMERICA. BULLETIN-1

GEOPHYSICAL RESEARCH LETTERS-?

HEALTH PHYSICS - 4

HIGH TEMPERATURE SCIENCE-2 
IAEC PROCESS DESIGN AND

DEVELOPMENT -1

IEEE JOURNAL OF QUANTUM

ELECTRONICS-2

IEEE TRANSACTIONS ON ACOUSTIC, SPEECH, AND SIGNAL PROCESSING-3

IEEE TRANSACTIONS ON COMPUTERS-I

IEEE TRANSACTIONS ON GEOSCIENCE ELECTRONICS-2

IEEE TRANSACTIONS ON MAGNETICS-1.

IEEE TRANSACTIONS ON NUCLEAR SCIENCE - 4

IEEE TRANSACTIONS ON PLASMA SCIENCE - I

IEEE TRANSACTIONS ON POWER

APPARATUS AND SYSTEMS-1

IEEE TRANSACTIONS ON SYSTEMS, MAN, AND CYBERNETICS-I

IMMUNOCHEMISTRY - I

INDIAN JOURNAL OF TECHNOLOGY-I

INORGANIC AND NUCLEAR CHEMISTRY LETTERS-1

INORGANIC CHEMISTRY-5

INTERNATIONAL JOURNAL OF APPLIED RADIATION AND ISOTOPES-2

INTERNATIONAL JOURNAL OF NUCLEAR MEDICINE AND BIOLOGY-1

INTERNATIONAL JOURNAL OF RADIATION ONCOLOGY, BIOLOGY AND PHYSICS-3
JOURNAL OF THE AIR POLLUTION CONTROL ASSOCIATION-1

JOURNAL OF THE AMERICAN CHEMICAL SOC IE TY -4

JOURNAL OF APPLIED MATHEMATICAL PHYSICS-1

JOURNAL OF APPLIED MECHANICS-I

JOURNAL OF APPLIED PHYSICS-1.4

JOURNAL OF THE BRITISH

INTERPLANETARY SOCIETY-I

JOURNAL OF CHEMICAL AND

ENGINEER ING DATA-I

JOURNAL OF CHEMICAL PHYSICS- 47

JOURNAL OF COMBINATIONAL THEORY-I

JOURNAL OF COMBINATORICS, INFORMATION AND SYSTEM SCIENCES-1

JOURNAL OF COMPUTATIONAL PHYSICS-4 JOURNAL OF DIFFERENTIAL EQUATIONS-1

JOURNAL OF ENVIRONMENTAL SCIENCE AND HEALTH-I

JOURNAL OF GENERAL VIROLOGY-1

JOURNAL OF GEOMAGNATISM AND GEOELE TRICITY-1

JOURNAL OF GEOPHYSICAL RESEARCH-20

JOURNAL OF HISTOTECHNOLOGY -1

JOURNAL OF IMMUNOLOGY-3

JOURNAL OF INORGANIC AND NUCLEAR C.HEMISTRY -5 
JOURNAL OF LABELLED COMPOUNDS AND RADIOPHARMACEUT ICALS-1

JOURNAL OF THE LESS-COMMON

METALS-1

JOURNAL OF LOW TEMPERATURE

PHYSICS-2

JOURNAL OF MATHEMATICAL BIOLOGY-2.

JOURNAL OF MATHEMATICAL PHYSICS-1

JOURNAL OF MOLECULAR

SPECTROSCOPY - 3

JOURNAL OF MOLECULAR STRUCTURE-1

JOURNAL OF THE NATIONAL CANCER

INST I TUTE-2

JOURNAL OF NUCLEAR MATERIALS-7

JOURNAL OF NUCLEAR MEDICINE-1

JOURNAL OF OPTIMIZATION THEORY AND APPLICATIONS-2

JOURNAL OF THE OPTICAL SOCIETY OF AMERICA - 2

JOURNAL OF ORGANIC CHEMISTRY-I

JOURNAL OF PHOTOCHEMISTRY-3

JOURNAL OF PHYSICAL CHEMISTRY-1

JOURNAL OF PHYSICS AND CHEMISTRY OF SOLIDS-1

JOURNAL OF PHYSICS. B. (PROCEEDINGS DF THE PHYSICAL SOC IETY ) -2

JOURNAL OF THE PHYSICAL SOCIETY OF JAPAN-1
JOURNAL OF PHYSIQUE-I

JOURNAL OF PLASMA PHYSICS-I

JOURNAL OF PRESSURE VESSEL

TECHNOLOGY - I

JOURNAL OF QUANTITATIVE

SPECTROSCOPY \& RADIATIVE

TRANSFER-3

JOURNAL OF STATISTICAL

COMPUTATION AND SIMULATION-1

JOURNAL OF STATISTICAL PHYSICS-1

JOURNAL OF VACUUM SCIENCE AND TECHNOLOGY -4

JOURNAL OF VOLCANOLOGY AND

GEOTHERMAL RESEARCH-1

LASER FOCUS-1

MATERIALS EVALUATION-I

METALLURGICAL TRANSACTIONS-1

NUCLEAR FUSION-?

NUCLEAR INSTRUMENTS AND METHODS-7

NUCLEAR MATERIALS MANAGEMENT-8

NUCLEAR PHYSICS- 6

NUCLEAR SCIENCE AND ENGINEERING-9

NUCLEIC ACIDS RESEARCH - I

OPTICAL ENGINEERING-1

OPTICS COMMUNCATIONS-2

OPTICS LETTERS-10

PHYSICAL REVIEW-72 
PHYSICAL REVIEW LETTERS-20

PHYSICS IN MEDICINE AND BIOLOGY-2

PHYSICS LETTERS---9

PHYSICS OF FLUIDS-15

PHYSICS TEACHER-1

PROCEEDINGS OF THE AMERICAN

MATHEMATICAL SOCIETY-I

PROPELLANTS AND EXPLOSIVES-1

PUBLICATIONS OF THE ASTRONOMICAL

SOCIETY OF THE PACIFIC-I

RADIATION RESEARCH-3

RADIOLOGY - 1

REVIEW OF SCIENTIFIC INSTRUMENTS- 6

REVIEWS OF MOOERN PHYSICS-1

ROCKY MOUNTAIN JOLRNAL OF

MATHEMATICS -2

SCIENCE-2

SCRIPTA METALLURGICA-2

SIAM JOURNAL ON MATHEMATICAL

ANALYSIS-1

SKY AND TELESCOPE-I

SOLID STATE COMMUNICATIONS-5

SURFACE SCIENCE-3

TECHNOMETRICS-1

TRANSPORT THEORY AND STATISTICAL PHYSICS -1
WATER, AIR, AND SOIL POLLUTION-1

WELDING JOURNAL-1

B. BOOKS -3

C. CHAPTERS OF BOOKS AND ENCYCLOPEDIA ART ICLES-14

D. PAPERS PUBLISHED IN CONFERENCE PROCEEDINGS-85

E. U.S. PATENTS-4

F. LOS ALAMOS SCIENTIFIC LABORATORY REPORTS -435

G. PAPERS ISSUED AS LASL REPORTS BY TIC AND NTIS-197

H. THESES-14 